\title{
Werte in umweltgeschichtlichen Texten
}

Eine Analyse anhand des Begriffs Umweltschaden

\author{
Dissertation \\ zur Erlangung des Doktorgrades \\ der Fakultät für Agrarwissenschaften \\ der Georg-August-Universität Göttingen
}

vorgelegt von

Sonja Schumacher

geboren in Rinteln

Göttingen, Juli 2017 
1. Referent:

Prof. Dr. Rainer Marggraf

Georg-August-Universität Göttingen

Department für Agrarökonomie und Rurale Entwicklung

Abteilung Umwelt- und Ressourcenökonomik

2. Korreferent: $\quad$ Prof. Dr. Heiko Faust

Georg-August-Universität Göttingen

Geographisches Institut

Abteilung Humangeographie

Tag der mündlichen Prüfung: 13.07.2017 
Danksagung

An dieser Stelle möchte ich mich bei allen bedanken, die mir die Erstellung meiner Dissertation ermöglicht haben.

Herr Professor Marggraf hat mich als erster Gutachter stets mit seinen Anregungen unterstützt, und auch Herrn Professor Faust bin ich für sein zweites Gutachten zu Dank verpflichtet. Ohne ihre geduldige Unterstützung und ihren wertvollen akademischen Rat wäre diese Arbeit nicht entstanden. Bedanken möchte ich mich auch für die Freiheit, die sie mir während des gesamten Forschungsprojektes gewährten, was maßgeblich zum Gelingen dieser Arbeit beitrug.

Mein besonderer Dank gilt meiner Familie. 


\section{Inhaltsverzeichnis}

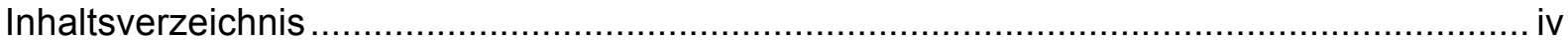

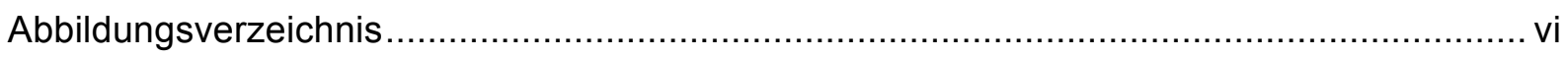

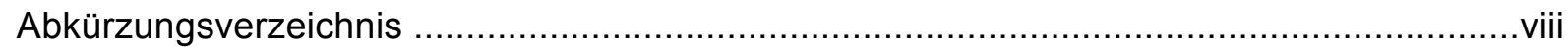

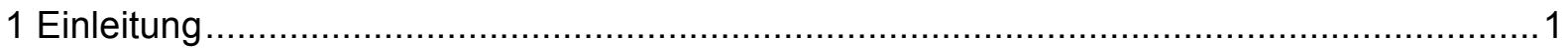

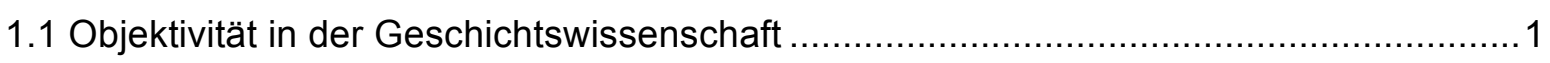

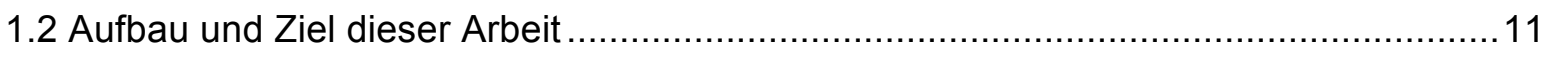

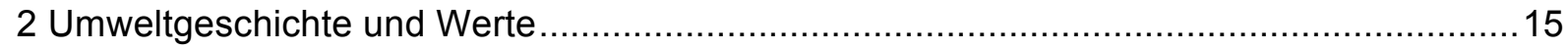

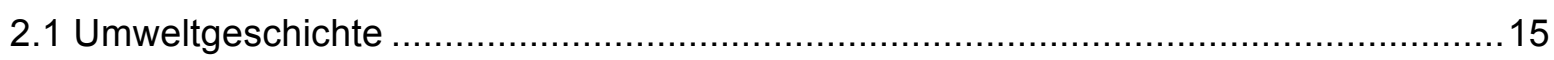

2.1.1 Entstehung der Disziplin Umweltgeschichte ................................................15

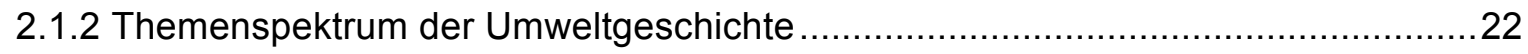

2.1.3 Verbindung natur- und geisteswissenschaftlicher Methoden ..............................26

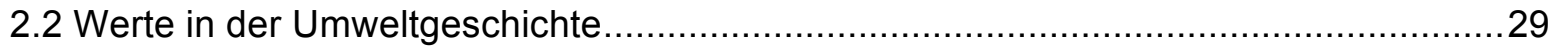

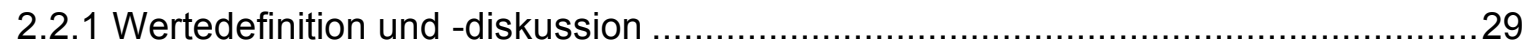

2.2.2 Forscherpositionen in verschiedenen Epochen und Disziplinen ..........................36

2.2.3 Werteidentifikation, insbesondere von impliziten Werten .................................... 41

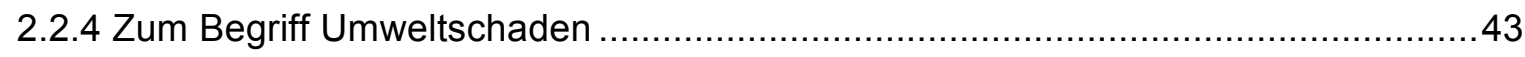

2.2.5 Wertearten und der anthropozentrische Ansatz ......................................... 50

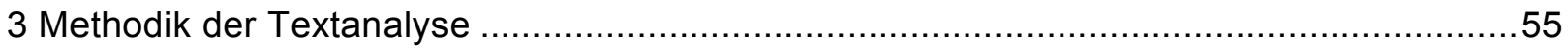

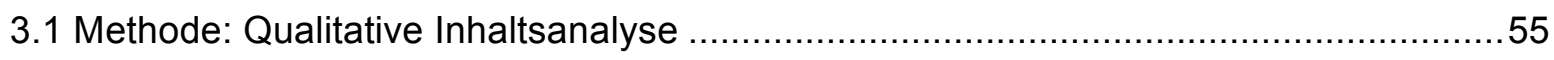

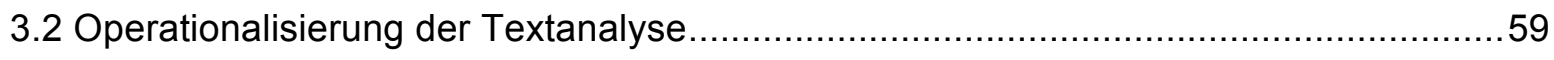

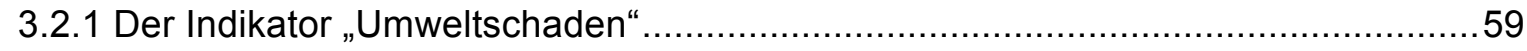

3.2.2 Ableitung der zugrundeliegenden Werte ..................................................... 61

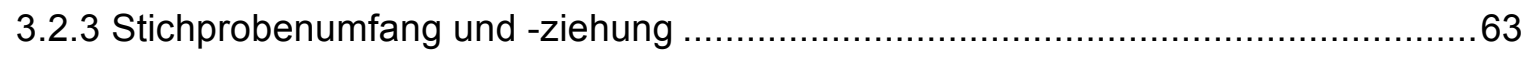

3.2.4 Auswahlkriterien der umweltgeschichtlichen Texte.........................................65

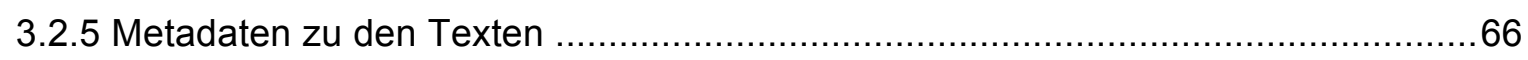

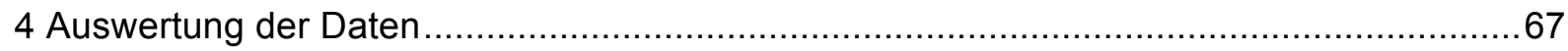

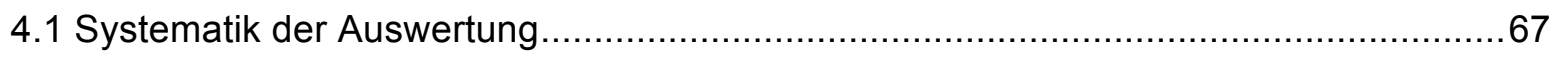

4.2 Vorstellung Indikatoren und Wertekategoriensystem ....................................67 


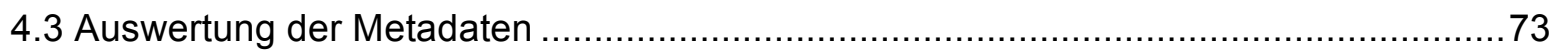

4.4 Quantitative Auswertungen zu Indikator- und Wertecodierungen ...............................76

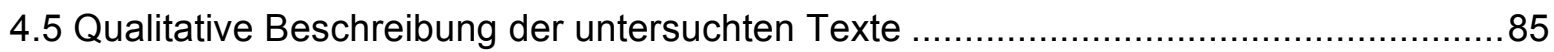

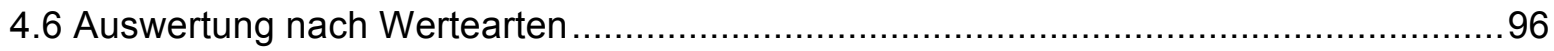

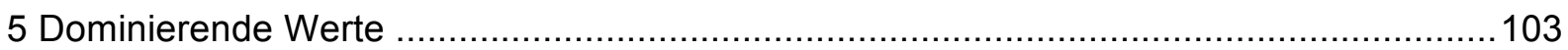

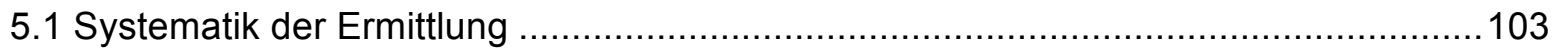

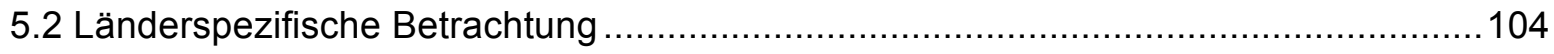

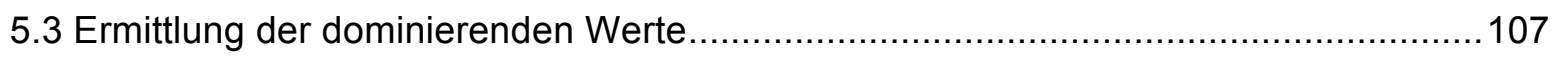

5.4 Einzelbetrachtung der dominierenden Werte ........................................................ 108

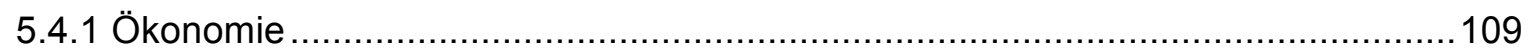

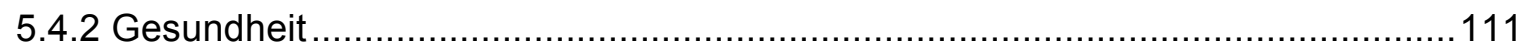

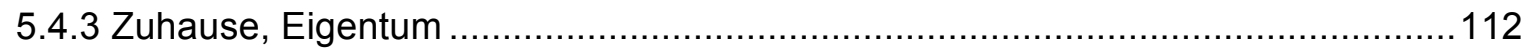

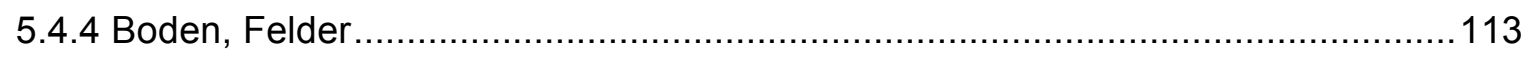

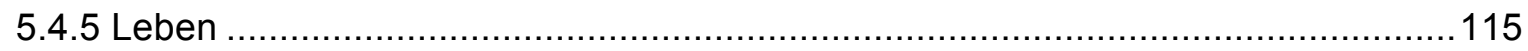

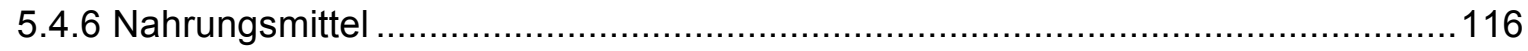

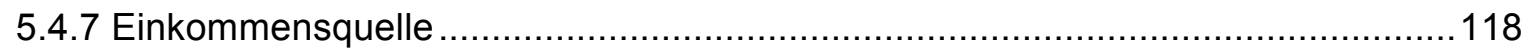

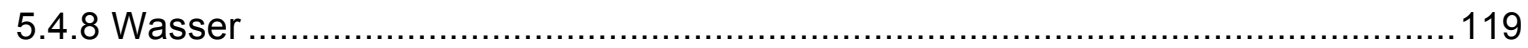

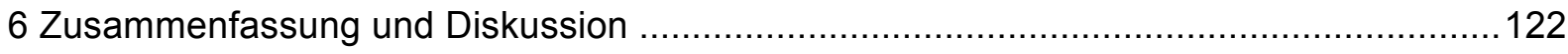

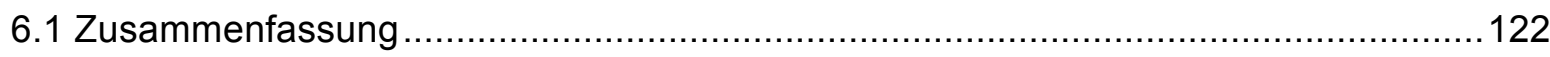

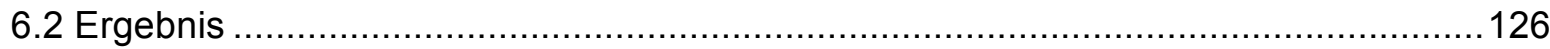

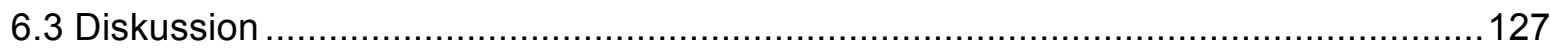

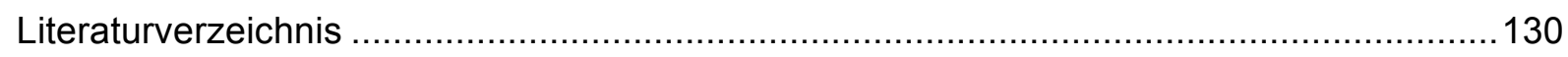

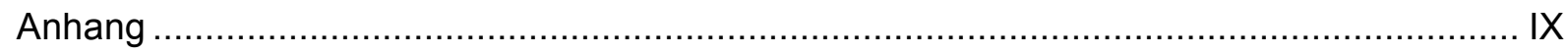

A Hochschulen in Deutschland mit Geschichte als Studiengang .................................. IX

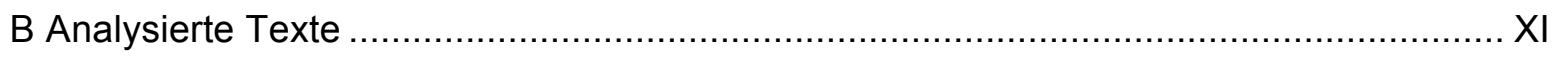

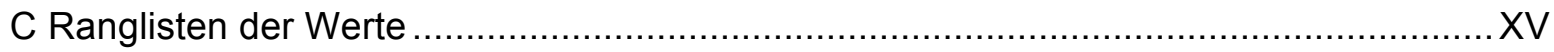

D Wertekategoriegruppen - Zuordnung der Werte .............................................. XVIII

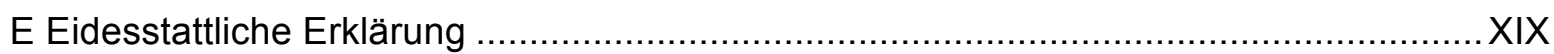




\section{Abbildungsverzeichnis}

Abbildung 1: Ansätze zur Strukturierung der Umweltgeschichte .................................21

Abbildung 2: Wertekategorien (Ökologische Ökonomie) …............................................49

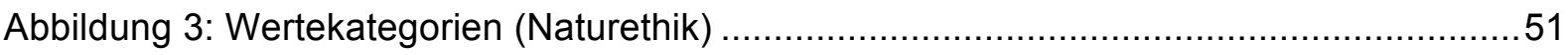

Abbildung 4: Ablaufdiagramm der qualitativen Inhaltsanalyse dieser Arbeit........................58

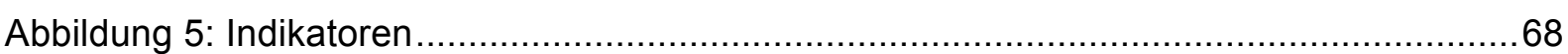

Abbildung 6: Wertekategorien (aus der Textanalyse dieser Arbeit) ....................................69

Abbildung 7: Jahr der Veröffentlichung mit jeweiliger Anzahl der Texte ..............................73

Abbildung 8: Land der Hochschule mit jeweiliger Anzahl der Texte ...................................74

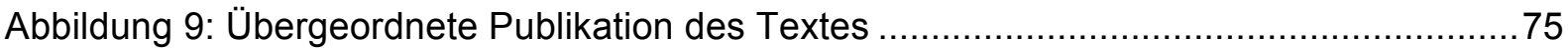

Abbildung 10: Indikatoren (nach Anzahl Codierungen) …...............................................

Abbildung 11: Anzahl Indikatoren (nach Anzahl Texte) ......................................................77

Abbildung 12: Anzahl Codierungen pro Text (Indikator- zu Wertecodierungen) ....................78

Abbildung 13: Anzahl Codierungen pro Text (nach Jahr der Veröffentlichung) .....................79

Abbildung 14: Durchschnittliche Anzahl Codierungen pro Text (nach Sprache bzw. Land) ..80

Abbildung 15: Anzahl Codierungen pro Text (nach Medium bzw. Universität) ......................81

Abbildung 16: Anzahl Wertecodierungen und der Indikator „Umweltschaden“ .....................82

Abbildung 17: Quantifizierte Wertecodierungen (nach Anzahl Codierungen) ......................83

Abbildung 18: Quantifizierte Werte (nach Anzahl Codierungen) ...................................... 84

Abbildung 19: Quantifizierte Wertecodierungen (nach Anteil Dokumente) ..........................85

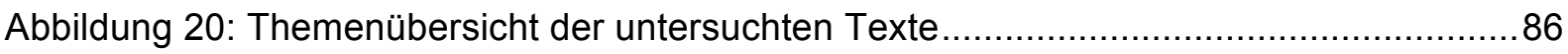

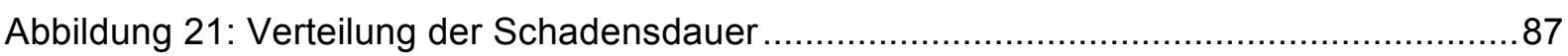

Abbildung 22: Ereignis- und Schadensausdehnung (Fläche) …................................... 88

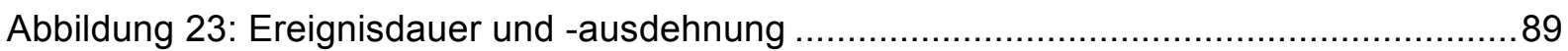

Abbildung 24: Schadensursachen (nach Anteil Dokumente) ...........................................90

Abbildung 25: Ereignisdauer/-ausdehnung und Schadensdauer ......................................

Abbildung 26: Beispiele Dokumentenporträts (links: Josephson 2010, rechts: Bayerl 2009) 92

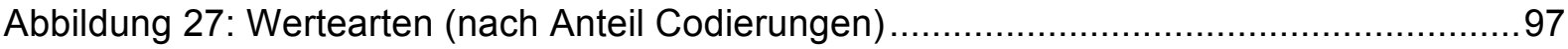

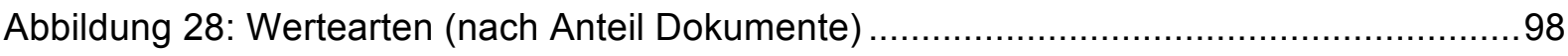

Abbildung 29: Existenzialwerte (nach absoluter Anzahl Codierungen) ................................99

Abbildung 30: Existenzialwerte (nach Anteil Dokumente und Ländern).............................100

Abbildung 31: Use / Non-Use Values (nach Anteil Codierungen) ..................................... 101

Abbildung 32: Use / Non-Use Values (nach Anteil Dokumente) .......................................101

Abbildung 33: Werteranglisten nach Anteil Codierungen und Anteil Dokumente ................103

Abbildung 34: Dominierende Werte - Texte aus Deutschland ........................................... 105

Abbildung 35: Dominierende Werte - Texte aus den USA ......................................... 106 


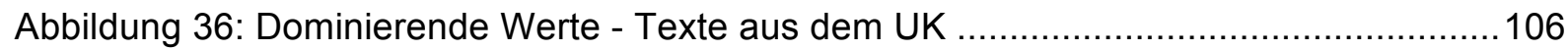

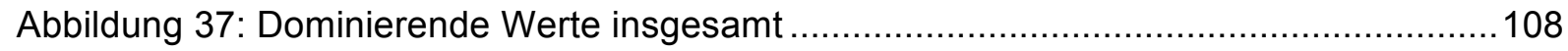

Abbildung 38: Der Wert Ökonomie im Ländervergleich ..................................................110

Abbildung 39: Der Wert Gesundheit im Ländervergleich ............................................ 112

Abbildung 40: Der Wert Zuhause, Eigentum im Ländervergleich ....................................113

Abbildung 41: Der Wert Boden, Felder im Ländervergleich ............................................. 115

Abbildung 42: Der Wert Leben im Ländervergleich................................................... 116

Abbildung 43: Der Wert Nahrungsmittel im Ländervergleich ...........................................118

Abbildung 44: Der Wert Einkommensquelle im Ländervergleich .....................................119

Abbildung 45: Der Wert Wasser im Ländervergleich .........................................................120 
Abkürzungsverzeichnis

$\mathrm{AB} \quad$ Artenbestände

A. d. V. Anmerkung des Verfassers

ASEH American Society for Environmental History

AV Artenvielfalt

BO Boden, Felder

EF Erholung, Freizeit, Komfort

EQ Einkommensquelle

ER Ernte, Saatgut

ESEH European Society for Environmental History

GE Gesundheit

Hervorh. i. O. Hervorhebung im Original

HU Hunger

ID Identität

IM Imageschaden

IS Infrastruktur

KR Krankheit, Verletzungen

LG Lebensgrundlage

LU Luft

MAXQDA Software zur computergestützten qualitativen Daten- und Textanalyse

MO Moralische Verantwortung, Pflicht

NA Nachhaltigkeit

NA als Untergruppe von LG: Nahrung

NM NM - Nahrungsmittel

ÖK Ökonomie

ÖS Ökosystem

RE Religion, Glaube

RL Rohstofflieferant

SCÖ Schönheit der Natur

ST Stabilität (polit.)

TI Tiere

TO Tod

TR Tradition

UN Unberührte Natur

WA Wasser

WI Wissen, Erkenntnis

ZAA Zeitschrift für Agrargeschichte und Agrarsoziologie

$\mathrm{ZH} \quad$ Zuhause, Eigentum 


\section{Einleitung}

\subsection{Objektivität in der Geschichtswissenschaft}

Die heutige Geschichtswissenschaft kennt viele Untersuchungsgegenstände und einen ebenso vielfältigen Methodenbaukasten. Dieses methodische Repertoire hat dazu beigetragen, dass die Geschichtsschreibung zunehmend faktenbasiert, nachprüfbar und nicht zuletzt auch objektiver, zumindest aber intersubjektiv nachvollziehbarer ${ }^{1}$ geworden ist. Nichtsdestotrotz ist die Geschichtswissenschaft eng mit der Hermeneutik verbunden. Bei der Interpretation ergibt sich ein gewisser Spielraum, der allerdings auch in anderen Disziplinen existiert. Allein die Physik scheint ohne solche "Graubereiche“ auszukommen. „Stegmann macht z.B. darauf aufmerksam, dass wir bei der Beschreibung der Wirksamkeit von Genen auf die Begriffe ,richtig' oder ,falsch“ nicht verzichten können. Ein Gen kann ,richtig' oder ,falsch' abgelesen werden und führt dann zum funktionalen oder dysfunktionalen Verhalten oder Aufbau von Lebewesen. In der Physik kommen solche Begriffe nicht vor. Ein Stein kann nicht ,richtig' oder ,falsch' zur Erde fallen. Wo wir diese Begriffe gebrauchen, muss es einen Wert geben, der dieser Unterscheidung erst ihren Sinn verleiht." ${ }^{2}$ Auch Stern weist darauf hin, dass die Bewertung eines Verhaltens als "gut" oder „böse“ die Existenz und Akzeptanz einer Norm voraussetzt, an der das fragliche Verhalten gemessen werden kann. ${ }^{3}$ Der folgende Abschnitt soll die Schwierigkeiten einer auf Objektivität zielenden Geschichtswissenschaft beleuchten.

Historikern fällt bei ihrer Arbeit eine große Verantwortung zu. Zunächst wählt der Forscher seinen Untersuchungsgegenstand aus einer großen Menge möglicher Fragestellungen aus. Bereits hier stellt sich die Frage, weshalb er gerade diesen Untersuchungsgegenstand gewählt hat. ${ }^{4}$ Warum ist dieser Gegenstand den Arbeitsaufwand wert, andere aber nicht? Goertz stellt hierzu fest, dass die Selektion des Gegenstandes abhängig von den Werten ist, denen sich der Forscher verpflichtet fühlt. ${ }^{5}$ Im Folgenden wird sich der Historiker überlegen

\footnotetext{
1 Hedinger kommt diesbezüglich zu dem ernüchternden Schluss, dass „bisher keine Möglichkeit allgemeingültiger, intersubjektiv verbindlich prüfbarer Normen und Wertungen verläßlich sichtbar geworden“"ist. Hans-Walter Hedinger, Subjektivität und Geschichtswissenschaft: Grundzüge einer Historik. Univ., Diss.-Hamburg, 1967, Berlin, 1969, S. 637-638.

2 Hans-Dieter Mutschler, Gibt es Werte in der Natur?, in: Marie Luisa Allemeyer/Katharina Behrens/Katharina Ulrike Mersch (Hrsg.), Eule oder Nachtigall? Tendenzen und Perspektiven kulturwissenschaftlicher Werteforschung, Göttingen 2007, S. 221-239, hier S. 229.

${ }^{3}$ Vgl. Alfred Stern, Geschichtsphilosophie und Wertproblem, München (u.a.) 1967, S. 250.

${ }^{4}$ Falls der Forscher sein Thema selbst auswählt, muss es inm zuvor bekannt sein. Eine mögliche Ideenquelle ist die Zeitungslektüre. Darüber, wie Zeitungsmeldungen über Umweltschadensfälle auf den Leser wirken und welche kognitiven Bewertungen sie erzeugen vgl. die Untersuchung von Pfeiffer et al. (Till Pfeiffer/Sylvia Manz/Josef Nerb, Wer den Schaden macht, hat auch das Wissen: Kohärenzeffekte der kognitiven und emotionalen Bewertung von Umweltschadensfällen, in: Zeitschrift für Psychologie (2005) 213 (1), S. 44-58).

${ }^{5}$ Hans-Jürgen Goertz, Umgang mit Geschichte: Eine Einführung in die Geschichtstheorie, Reinbek bei Hamburg, 1995, S. 137
} 
wie er die zu untersuchende Problemstellung beschreibt. Üblicherweise formuliert er hierzu eine oder mehrere Forschungsfragen. Auch hier ist die Frage zu stellen, weshalb der Historiker gerade die von ihm gewählten Aspekte untersuchen möchte. Beide Auswahlprozesse werden anscheinend selten dokumentiert oder in wissenschaftliche Abhandlungen einbezogen. Vielmehr hat sich bei den Recherchen für diese Arbeit gezeigt, dass Werte in den Geschichtswissenschaften zwar als Untersuchungsgegenstand existieren, jedoch anscheinend selten weder die Werte, die implizit in einem Text bzw. einem Untersuchungsgegenstand enthalten sind, noch die Werte, die seitens des Forschenden in die Forschungsarbeit einfließen, ausreichend von den Forschenden reflektiert und in ihren Ausarbeitungen berücksichtigt werden. ${ }^{6}$ Falls doch, so wird diese Reflexion nicht dokumentiert und ist damit nicht intersubjektiv nachvollziehbar. Dies wird z. B. von Rickert als Mangel erkannt, der sogar noch einen Schritt weiter ging und nicht nur zur Reflexion der eigenen Werte mahnt, sondern darüber hinaus eine Begründung zur Gültigkeit dieser Werte fordert: „Wer Kulturwissenschaft treiben will im höchsten Sinne des Wortes, (...) wird auf die Notwendigkeit geführt, sich auf seine leitenden Kulturwerte zu besinnen und ihre Geltung zu begründen. Das Arbeiten mit unbegründeten Wertsetzungen würde in der Tat der Wissenschaft widersprechen." 7

Die Kenntnis der Werte des Forschenden kann auch hilfreich sein, wenn verschiedene Forscher bei identischer Datenlage zu unterschiedlichen Schlussfolgerungen kommen. „Denn die Beurteilung ein- und desselben Gegenstandes könne durchaus changieren, wenn der Gegenstand von unterschiedlichen Personen eingeschätzt wird. (...) Wiederum wird deutlich, daß Wertvorstellungen nicht als feststehende Leitbilder, sondern als dynamische Bestandteile gesellschaftskonstituierender Prozesse zu deuten sind. “8 Die hier von Mersch angesprochene Dynamik ist insbesondere in den Geschichtswissenschaften eine Herausforderung, da sich die Forschung respektive der zeitliche Abstand zwischen Ereignis und Erforschung durchaus über eine längere Zeitspanne erstrecken kann. In diesem Zusammenhang ist auch die individuelle Erfahrung des Forschers zu nennen, die die Interpretation des Untersuchungsgegenstandes beeinflusst. Schleiermacher forderte einst, dass sich der Forscher in den Autor hineinversetzen müsse, um sein Werk zu verstehen. Ebendies wird durch die bereits vorhandenen Erfahrungen erschwert; der Historiker weiß mehr als der Autor zu der Zeit als er den Text verfasste. Dieses Wissen um zukünftige Ereignisse wird sich kaum ausblenden lassen, sodass es dem Forscher unmöglich ist, sich

\footnotetext{
${ }^{6}$ Hier ist die Differenzierung zwischen Werten des Untersuchungsgegenstandes und den Werten des Forschers, die seine Arbeit beeinflussen, wichtig. Im Folgenden geht es um die Werte des Forschers.

7 Heinrich Rickert/Friedrich Vollhardt, Kulturwissenschaft und Naturwissenschaft, Stuttgart, 1986, Lizenzausg, S. 170.

${ }^{8}$ Katharina Ulrike Mersch, Überlegungen zum Wert historischer Werteforschung, in: Marie Luisa Allemeyer /Katharina Behrens/Katharina Ulrike Mersch (Hrsg.), Eule oder Nachtigall? Tendenzen und Perspektiven kulturwissenschaftlicher Werteforschung, Göttingen 2007, S. 9-34, hier S. 17.
} 
gänzlich in den Wissensstand des Autors zurückzuversetzen. Gerade dieses Wissen um die Schwierigkeiten des Hineinversetzens aber ist es, das eine Annäherung ermöglicht. Indem der Forscher sich bewusst macht, was der Autor wissen und was er nicht wissen konnte, nähert er sich den Hintergründen des Verfassers an. Ein äußerst positiver Nebeneffekt dieser Art der Annäherung besteht darin, dass sich der Forschende seiner eigenen Bewertungen bewusst werden kann. „Der Historiker (...) gehört einer bestimmten Nation, Klasse, Schicht, Berufsgruppe und so weiter an, mit allen daraus folgenden Konsequenzen der von inm (am häufigsten unbewußt) akzeptierten Stereotype (...).“9 Aus diesen Zugehörigkeiten ergeben sich natürlicherweise verschiedene verinnerlichte Werthaltungen. „Tatsächlich schlagen sich soziale Werte bevorzugt auf andere Weise nieder. Sie wirken gleichsam von innen und entfalten ihren Einfluss ganz ohne Zwang in den Köpfen der beteiligten Wissenschaftler. Soziale Werte kommen insbesondere dann zum Ausdruck, wenn starke gesellschaftliche Einstellungen auf eine unklare Datenlage treffen."10 Aber auch wenn eine Interpretation aus anderen Gründen als einer unklaren Datenlage erforderlich ist, spielen die Werte des Forschers eine Rolle. Und dass die Untersuchungsgegenstände der Geschichtswissenschaften fast immer der Interpretation bedürfen, dürfte unstrittig sein. Allerdings lassen sich Wertvorstellungen nicht direkt aus der Zugehörigkeit zu einer bestimmten Schicht, Klasse etc. ableiten. Reusswig schreibt mit Bezug auf die Sozialwissenschaften, dass die Vorhersage von individuellen Einstellungen vor dem Hintergrund der klassischen sozialen Statusgruppen u. a. aufgrund der Pluralisierungen der Wertevorstellungen schwieriger werde. ${ }^{11}$

Bevor wir uns aber der Frage weiter nähern, welche Werte in einen Text einfließen, stellt sich zunächst eine andere Frage: Wie lässt sich das Streben nach Objektivität in der Praxis umsetzen? Husserls Phänomenologie bietet eine methodische Möglichkeit und lässt sich wie folgt zusammenfassen: „Phänomenologisches Vorgehen bedeutet, einen Gegenstand so objektiv wie möglich zu beschreiben, d.h. inn zu befreien von subjektiven, theoretischen und traditionellen Elementen, damit sein Wesen zur Geltung kommen kann."12 Anders gesagt, man müsste die Geschichtswissenschaft um (fast) alles bereinigen, was ihren Erkenntnisgewinn ausmacht: Ohne die Interpretation von Daten in ihrem jeweiligen historischen Kontext und die Einordnung in einen größeren Zusammenhang dürften die zu erwartenden Schlussfolgerungen jedoch eher trivial sein. Die Alltagstauglichkeit der Phänomenologie darf für diesen Anwendungsbereich also angezweifelt werden. Vielmehr

\footnotetext{
${ }^{9}$ Adam Schaff, Geschichte und Wahrheit, Wien, 1970, S. 237.

${ }^{10}$ Martin Carrier, Wissenschaftstheorie zur Einführung, Hamburg, 2008, 2., überarb, S. 168.

${ }^{11}$ Vgl. Fritz Reusswig, Umweltgerechtes Handeln in verschiedenen Lebensstil-Kontexten, in: Volker Linneweber /Elisabeth Kals (Hrsg.), Umweltgerechtes Handeln. Barrieren und Brücken, Berlin 1999, S. 49-67, hier S. 52.

12 Siegfried Lamnek, Qualitative Sozialforschung: Lehrbuch, Weinheim, 2005, S. 58.
} 
muss es das Ziel eines Historikers sein, möglichst genau zu arbeiten und dies für andere nachvollziehbar zu dokumentieren. Hierzu zählt sicher auch die Zuhilfenahme naturwissenschaftlicher Analysemethoden zur Sicherung bzw. Bestimmung von Quellen oder Artefakten. Gerade dieser Hang zur Genauigkeit, das hingebungsvolle Streben, jedes kleine Detail korrekt zu erfassen, macht einen guten Historiker aus. Rickert hat dies einst sehr schön formuliert: „Und wer also nicht die Fähigkeit besitzt, sich einmal (...) hineinzusteigern in die Vorstellung, daß das Schicksal seiner Seele davon abhängt: ob er diese, gerade diese Konjektur an dieser Stelle dieser Handschrift richtig macht, der bleibe der Wissenschaft ja nur fern." ${ }^{13}$ An dieser Stelle erscheint es wichtig zu betonen, dass Leidenschaft nicht zwangsläufig konträr zu Objektivität bzw. dem Streben danach sein muss. Oder wie Tapper es zusammenfasst: „(...) good history cannot be written without compassion (...)“14.

Eine, wenn nicht die Hauptaufgabe des zeitgenössischen Historikers besteht darin, verlässliche Quellen zu finden. Jede Quelle aber ist voreingenommen und muss innerhalb ihres Kontextes, d. h. innerhalb der Rahmenbedingungen ihrer Entstehungszeit, interpretiert werden. „Unser ganzes Handwerk gründet sich eben auf die Einsicht, daß unser Wissen über irgendein Ereignis durch Quellen zu uns gelangt, von denen wir wissen, daß sie nicht perfekte Widerspiegelungen von ,Realität" sind.“" ${ }^{15}$ Auch Castelli hebt den besonderen Stellenwert der Kontextbeachtung für Historiker hervor: „Es ist gerade die Geschichtswissenschaft, die lehrt, die Frage der Wahrheit mit Vorsicht zu behandeln und sie in ihren Bezügen zu all den verschiedenen Bereichen zu betrachten, die sie berührt." ${ }^{16}$

Bei der Untersuchung der Quellen kann der Historiker also nicht umhin, die vorhandenen Informationen zu interpretieren. Durch welche Brille aber blickt der Forscher auf die Fakten oder zumindest die Indizien? Und, vielleicht noch wichtiger, ist er sich bewusst, diese Brille zu tragen? Ist ein „brillenloser Blick“ überhaupt möglich? Werden bestimmte Informationen bevorzugt, andere ignoriert oder gar nicht erst recherchiert? Winiwarter schreibt hierzu: „HistorikerInnen haben einen blinden Fleck, von dem sie nur wissen können, dass er besteht, aber nicht, worin er besteht.“17 Der Historiker E.H. Carr beschreibt dieses Auswahlund Interpretationsdilemma humorvoll:

„Die Tatsachen ähneln wirklich nicht den Fischen auf dem Ladentisch des Fischhändlers, sondern vielmehr denen, die in einem weiten und oft unerreichbaren Ozean

\footnotetext{
${ }^{13}$ Max Weber, Wissenschaft als Beruf, 2006, S. 12.

${ }^{14}$ Alan Tapper, Is there an Ethics for Historians?, in: Cathie Clement (Hrsg.), Ethics and the practice of history, Perth, W.A. 2010, S. 16-36, hier S. 19.

${ }^{15}$ Martha Howell, Werkstatt des Historikers: Eine Einführung in die historischen Methoden, Köln, 2004, S. 187.

${ }^{16}$ Enrico Castelli Gattinara, Wahrheit, in: Anne Kwaschik/Mario Wimmer (Hrsg.), Von der Arbeit des Historikers. Ein Wörterbuch zu Theorie und Praxis der Geschichtswissenschaft, Bielefeld 2010, S. 215-218, hier S. 217.

${ }^{17}$ Verena Winiwarter/Martin Knoll, Umweltgeschichte: Eine Einführung, Köln (u.a.), 2007, S. 84.
} 
umherschwimmen; und was der Historiker fängt, wird zum Teil vom Zufall, in der Hauptsache aber davon abhängen, welchen Teil des Ozeans und welches Werkzeug er zum Fischen wählt, wobei natürlich beides durch die Art der Fische, die er zu fangen wünscht, bestimmt ist. Im großen und ganzen wird der Historiker der Art Tatsachen habhaft werden, auf die es inm ankommt. Geschichte bedeutet Interpretation." ${ }^{18}$

„Die Arbeit des Historikers ist (...) Synthese und Hypothese zugleich: Synthese insofern, als sie die Rekonstruktion eines ganzheitlichen Bildes aufgrund der Kenntnis einzelner Fakten anstrebt; Hypothese aber insofern, als die zwischen diesen Fakten festgestellten Relationen niemals ganz und gar offensichtlich und verifizierbar sind."19 Auch Hedinger gelangte zu dieser Schlussfolgerung: „Historische Aussagen gelangen nie über ein Höchstmaß an Wahrscheinlichkeit hinaus. (...) Der Grad der Wahrscheinlichkeit ist allerdings nicht präzise angebbar.“20 Diese Feststellung wird nur ein wenig gemildert, als Hedinger dies auch für die Naturwissenschaften konstatiert, wenngleich in geringerem Maße. Interpretation ist Kunst und Wissenschaft, Intuition und Technik, unterliegt aber zugleich dem Einfluss des Zeitgeistes. Auch die Geschichtswissenschaft unterliegt den vorherrschenden geistigen Strömungen. „Ebenso wichtig wie soziale und politische Entwicklungen, wenn nicht wichtiger, waren für den Wandel der Geschichtsschreibung jedoch Geistesströmungen, die die Art, wie Historiker über Quellen denken, sie lesen und interpretieren, grundlegend verändert haben." ${ }^{21}$ Auch die Fragen der Historiker werden stets im Horizont ihrer Zeit gestellt und verlangen daher immer nach kritischer Reflexion ihres Standpunktes, des Erkenntnisziels, des Blickwinkels und der Methodik. Und nicht nur die aktuellen wissenschaftlichen Strömungen beeinflussen die Art der Interpretation, sondern natürlich auch der individuelle Horizont des Forschers. Gadamer beschreibt dieses Phänomen als kulturelle Horizontbedingtheit. Daraus folgert er, dass es eine „Naivität des historischen Objektivismus“22 ist, Objektivität in der Geschichtsschreibung zu erwarten.

„Der Gelehrte ist meistens in seinen Anschauungen und Haltungen infiziert von derartigen Phobien, Vorurteilen und Neigungen, die Ereignisse in einer Weise zu interpretieren und zu beurteilen, die für seine Epoche, Klasse, sein gesellschaftliches Milieu, seine Berufsgruppe und so weiter charakteristisch sind. Das schlägt sich ganz offensichtlich in seiner Weltanschauung nieder, in seiner Haltung und den Ansichten über gesellschaftliche Probleme, was seinerseits nicht ohne Einfluß auf die Sicht des historischen Prozesses

\footnotetext{
${ }^{18}$ Carr, Edward Hallett, zitiert nach Schaff, S. 195.

${ }^{19}$ Ebenda, S. 238.

${ }^{20}$ Hedinger, S. 628.

${ }^{21}$ Howell, Howell 2004, S. 112.

22 Hans-Georg Gadamer, Wahrheit und Methode: Grundzüge einer philosophischen Hermeneutik, Tübingen, 1960, S. 283.
} 
bleiben kann, auf die Konstruierung und Auswahl der historischen Tatsachen, ganz von ihrer Interpretation zu schweigen.“23 Auch Hays beschrieb den umfassenden Einfluss der Werte auf die wissenschaftliche Tätigkeit "Technical data and scientific inquiry are shaped by values; what is investigated, the research design (...), the monitoring system - all are heavily influenced by values." ${ }^{24}$ Daraus zieht Hays den Schluss: „It is often difficult to accept the notion that what appear to be technical choices are, in fact, value choices." ${ }^{25}$ Gerade deswegen ist es wichtig, die eigene „Brille“, durch die man die Welt sieht, möglichst gut zu kennen. Auch Howell hebt die Bedeutung dieser Kenntnisse für gelungene geschichtswissenschaftliche Arbeiten hervor: „(...) können wir gleichwohl nützliche geschichtliche Darstellungen verfassen, wenn wir unsere Vorlieben und Abneigungen, unsere Voreingenommenheit und Vorurteile erkennen und sie gewissenhaft mitbedenken. ${ }^{26}$

Zweifelsohne trägt die Kenntnis der Entstehungsgeschichte und der Meilensteine der Geschichtswissenschaft bzgl. der Wertethematik zu einer im Sinne der Objektivität verbesserten Interpretation bei. Dies erfordert in der Tat sehr umfangreiches Wissen, dessen Darstellung den Umfang dieser Arbeit sprengen würde. Nichtsdestotrotz sollen hier einige Etappen der Geschichtswissenschaft angerissen werden. Zum einen um die zeitliche Dimension der Wertediskussion darzustellen und zum anderen, um die Wichtigkeit dieses zentralen Themas (nicht nur für die Geschichtswissenschaft) zu verdeutlichen.

Der Ursprung westlicher Geschichtsschreibung wird dem Griechen Herodot (485-425 v.Chr.) zugeschrieben. Jedoch erst Leopold von Ranke (1795-1886) begründete die moderne Geschichtswissenschaft, indem er wissenschaftliche Methoden integrierte. Damit machte er die Geschichtswissenschaft zu dem, was sie heute darstellt. Er vertrat die Ansicht, dass die Geschichtswissenschaft Dinge so berichten sollte, „wie sie eigentlich gewesen“. Diese Ansicht teilt er mit Thukydides, der bereits um 400 v.Chr. schrieb: „Es möge nun jeder, Arzt oder Laie, über sie seine Meinung sagen, (...) ich will nur beschreiben, wie sie verlief.“" ${ }^{27}$ Das Streben nach möglichst objektiver Geschichtsschreibung wäre demnach fast so alt wie die (westliche) Geschichtsschreibung selbst. „Der (...) Wandel vom Geschichtsschreiber zum historischen Forscher erfuhr jedoch erst im Historismus des 19. Jahrhunderts seine eigentliche Ausprägung.“28 Der Historismus ${ }^{29}$ steht für die idealistische Begründung der

\footnotetext{
${ }^{23}$ Schaff, S. 244.

${ }^{24}$ Samuel P. Hays, Explorations in environmental history: Essays, Pittsburgh, Pa., 1998, S. 67.

${ }^{25}$ Ebenda, S. 68.

${ }^{26}$ Howell, Howell 2004, S. 186.

${ }^{27}$ Thukydides/Helmuth Vretska, Der Peloponnesische Krieg, Stuttgart, 2014, [Nachdr.], 2, $48,3$.

${ }^{28}$ Christoph Cornelißen/Gunilla-Friederike Budde, Geschichtswissenschaften: Eine Einführung, Frankfurt am Main, 2004, S. 14.

${ }^{29}$ Die Zeit des Historismus war etwa 1850-1930. Vertreter waren u. a. Ranke, Droysen, Dilthey, Windelband und Rickert (in zeitlicher Reihenfolge).
} 
Geschichtswissenschaft, womit in erster Linie die Annahme der Möglichkeit objektiver Geschichtserkenntnis gemeint ist. ${ }^{30}$

Ebenfalls im 19. Jahrhundert entstand der Positivismus den u. a. Auguste Comte (17981857 ) entwickelte. Diese Schule war der Auffassung, dass durch eine objektive Analyse objektives Wissen über Ursache und Wirkung erlangt werden kann. Positivistisch (und somit auch naturwissenschaftlich) orientierte Philosophen untersuchen alle Disziplinen darauf, in welchem Maß sie den Normen der Physik entsprechen. ${ }^{31}$ „Das positivistische Wissenschaftsideal (...) kann folgendermaßen formuliert werden: eine vollwertige Disziplin hat Forschungen zum Inhalt, die darauf gerichtet sind, Gesetzmäßigkeiten zu finden (streng allgemeine Aussagen), weshalb sie primär theorieorientiert ist; sie stellt Theorien auf und nimmt auf sie Bezug, sie bietet Kausalerklärungen (vom ,Warum'-Typ) auf der Basis wissenschaftlicher Gesetze an und kann ihre Aussagen experimentell überprüfen. “32 In den 1960er-Jahren wurde eine intensive Auseinandersetzung über Methoden und Werturteile in den Sozialwissenschaften geführt, die als Positivismusstreit bekannt wurde.

Im 20. Jahrhundert wurde sowohl der Historismus als auch der Positivismus entkräftet. Der Historismus lehnt den Fortschrittsgedanken der Aufklärung ab. Wenn aber jede Epoche individuell und gleichberechtigt betrachtet wird, bedeutet das auch, dass es keine absolut gültigen Werte geben kann. U. a. Troeltsch kritisierte diesen Aspekt des Historismus. Bezüglich des Positivismus betrachtete Popper die Verifikationsmethode als logisch widerlegt.

Das sogenannte Wertfreiheitspostulat dürfte eines der am ausführlichsten diskutierten Probleme der Sozialwissenschaften sein und berührt auch die Entwicklung der Geschichtswissenschaft. Wertfreiheit in der Wissenschaftstheorie ist die Anforderung an eine Aussage, frei von normativem Inhalt zu sein. Während einige der Meinung sind, dass sich dieses Ziel durch Trennung von Fakt und Bewertung erreichen lässt - „Ein Wissenschaftler soll deutlich machen, welche seiner Äußerungen Wertungen und welche seiner Äußerungen objektsprachliche, d. h. Sachaussagen, sind.“33 -, vertreten andere ihren Standpunkt der Unmöglichkeit von Wertfreiheit sehr deutlich. So schrieb Rickert über „(...) das gedankenlose Gerede von der ,reinen' Deskription (... “34 und Schaff suchte die Diskussion im Keim zu ersticken, indem er darauf hinwies, dass schon der Forscher als erkennendes Subjekt

\footnotetext{
${ }^{30} \mathrm{Vgl}$. ebenda, S. 14.

${ }^{31}$ Vgl. Jerzy Topolski, Die Wissenschaftlichkeit der Geschichtsschreibung und ihre Grenzen, in: Pietro Rossi (Hrsg.), Theorie der modernen Geschichtsschreibung, Frankfurt am Main 1987, Dt. Erstausg., 1. Aufl, S. 191217, hier S. 194.

32 Ebenda, S. 196-197.

33 Karl-Dieter Opp, Methodologie der Sozialwissenschaften: Einführung in Probleme ihrer Theoriebildung und praktischen Anwendung, Wiesbaden, 2002, S. 225.

${ }^{34}$ Rickert et al., S. 108.
} 
Subjektivität einbringt. „Die sogenannte reine Objektivität ist doch eine Fiktion, der subjektive Faktor wird allein durch die Tatsache des Vorhandenseins eines erkennenden Subjekts in die historische Erkenntnis hineingetragen. ${ }^{\text {‘35 }}$

Aber es ging bzw. geht nicht nur um die Möglichkeit einer wertfreien Wissenschaft, sondern auch darum, ob Wissenschaft wertfrei sein sollte. „Die Geschichte ist nicht und kann auch nicht ,reine' Beschreibung sein, und das Postulat einer Geschichtsschreibung, die das Geschehen darstellt, ,wie es eigentlich gewesen', ist (...) eine wissenschaftlich schädliche Fiktion.“36 Dies findet Ausdruck im Werturteilsstreit, der zu Beginn des 20. Jahrhunderts (um 1910) geführt wurde und bei dem es um das Verhältnis von (Sozial)Wissenschaften zu Politik ging. Weber und Sombart vertraten die Ansicht, dass Wissenschaft aus sich selbst heraus zu keinem Werturteil führen kann. Forschung und wertende Betrachtung müssen voneinander getrennt werden. Goldscheid stimmte dem zwar zu, wies aber darauf hin, dass eine normative Ökonomie sinnvoll und notwendig ist. In diesem Zusammenhang ist auch der Sein-Sollen-Fehlschluss von Hume zu nennen, der besagt, dass es prinzipiell nicht möglich ist von Faktenbeschreibungen auf Werturteile zu schließen.

Saussure (1857-1913) befasste sich u. a. mit Linguistik und Semiotik und gilt als Wegbereiter des linguistic turn. Anfang des 20. Jahrhunderts brachte der linguistic turn die Wende der Wissenschaft auf die Sprache bzw. der sprachlichen Vermittlungsformen als Untersuchungsobjekt. „Für die Geschichtswissenschaft forderte der linguistic turn eine Wendung von den historischen Fakten auf die Wahrnehmung und Deutung dieser Fakten.“37 So verwundert es nicht, dass interpretative Verfahren und die Geisteswissenschaften an Einfluss gewannen. Auch Panofsky (1892-1968) forschte im Bereich der Semiotik und meinte, dass Beschreibung und Bewertung eines Objektes nacheinander geschehen. In Anlehnung an Panofskys Ikonologie erfolgt auf der ersten Ebene eine rein phänomenale Benennung von Objekten oder Ereignissen. Erst auf der zweiten Ebene bringt der Betrachter sein kulturelles Kontextwissen ein.

Gadamer zeigte 1960, „daß die zeitliche Distanz der Historiker von den Texten, die sie untersuchen, ihre Lesarten fundamental anders ausfallen läßt als die eines Zeitgenossen. Historiker können gleichzeitig verstehen, was der zeitgenössische Zeuge nicht verstanden hätte und müssen gleichwohl den Text aus der Perspektive der Kultur lesen, in der sie selbst beheimatet sind.“38 1969 entwickelte Robert F. Berkhofer eine Methode, die sich auf die Analyse der Taten von Menschen konzentrierte. Er begründete diese Herangehensweise

\footnotetext{
${ }^{35}$ Schaff, S. 235.

${ }^{36}$ Ebenda, S. 199.

${ }^{37}$ Stefan Jordan, Theorien und Methoden der Geschichtswissenschaft, Paderborn, 2009, S. 187.

${ }^{38}$ Howell, Howell 2004, S. 129.
} 
damit, „daß der Historiker die mentale Welt der Vergangenheit nicht wiedererschaffen kann; das einzige was er tun kann, ist zu untersuchen, was diese Menschen taten, da Historiker viel zu viel von sich selbst in ihre Interpretation dessen hineinlegen, was die Menschen einer anderen Zeit dachten, oder fühlten."39 Clifford Geertz (1926-2006) befasste sich mit interpretativer Ethnologie und hinterließ die Methode der dichten Beschreibung. Der Forscher nimmt hierbei seine Rolle und Herangehensweise in Beschreibung und Interpretation auf. Geertz meinte, dass es keine „reinen Daten“ gebe, vielmehr sind in den Daten bereits das Wissen und die Erfahrung des Forschers enthalten. Er betonte die genaue Beobachtung und Dokumentation jedes Details.

Bei der Interpretation eines Phänomens ist die Bedeutung immer abhängig vom jeweiligen Kontext, wobei Kontext begrifflich eher weit zu fassen ist: sämtliche Rahmenbedingungen in zeitlicher, politischer, räumlicher, personeller und persönlicher Hinsicht sollten Berücksichtigung finden. Es wird deutlich, wie komplex interpretative Methoden sein können. Hinzu kommt, dass es zeitlich kaum konstante Begriffsbedeutungen geben dürfte. Darüberhinaus geht die historische Erkenntnistheorie davon aus, „dass nicht nur die wissenschaftlichen Grundbegriffe einem historischen Wandel unterliegen, sondern auch die Kategorien, unter denen gedacht und verstanden wird, was wissenschaftliches Wissen ausmacht.“40 „Ähnliches gilt nach Habermas auch für Werte, die in einer modernen demokratischen Gesellschaft nicht mehr einfach vorausgesetzt werden könnten, sondern kommunikativ ausgehandelt würden. Man bezeichnet das so ausgehandelte Wertesystem als ,Diskursethik'."41 Mit der Wandlung von Begriffsbedeutungen befasst sich auch die Begriffsgeschichte, in Person Reinhart Koselleck (1923-2006), der u. a. den individuellen Erfahrungshorizont einbezog. Begriffsgeschichte „untersucht das Begriffsverständnis in einzelnen Texten, versucht also zu verstehen, wie bestimmte Personen ihre jeweilige Wirklichkeit begrifflich gefasst haben. “42 Auch Howell weist darauf hin, welch großen Einfluss die Semantik auf das Verständnis historischer Texte hat: „Historiker können auch vieles über einen Text erfahren, indem sie ihre Aufmerksamkeit schlicht darauf lenken, wie ein Autor ein bestimmtes Wort gebraucht, dessen geschichtliche Signifikanz sie erkannt haben. “43 Dass dasselbe Wort zu verschiedenen Zeiten oder in verschiedenen Kontexten etwas anderes bedeuten kann, stellte auch Jaques Derrida (1942-2004) fest. Er meinte, dass Bedeutung niemals fest, sondern die Beziehung zwischen dem Bezeichneten und dem Bezeichnenden

\footnotetext{
${ }^{39}$ Ebenda, S. 117.

${ }^{40}$ Hans-Jörg Rheinberger, Historische Epistemologie, in: Anne Kwaschik/Mario Wimmer (Hrsg.), Von der Arbeit des Historikers. Ein Wörterbuch zu Theorie und Praxis der Geschichtswissenschaft, Bielefeld 2010, S. 103-105, hier S. 103.

41 Jordan, S. 189.

42 Ebenda, S. 125.

${ }^{43}$ Howell, Howell 2004, S. 126.
} 
arbiträr und gleitend ist. Derrida war einer der Vertreter des Poststrukturalismus (manchmal auch als epistemologische Krise der Anthropologie bezeichnet), der sich mit dem Verhältnis von Sprache und Wirklichkeit auseinandersetzt und der Ende der 1960er-Jahre großen Einfluss auf die Geschichtswissenschaften ausübte. Demnach bildet Sprache die Realität nicht nur ab, sondern formt sie gleichzeitig auch. Ein weiterer Vertreter dieses Ansatzes war Michel Foucault (1926-1984), für den „(...) der realitätskonstituierende Charakter von Sprache im Vordergrund [steht]. (...) Foucault geht davon aus, dass Sprache nicht Realitäten abbildet, sondern schafft.“44 „In seinen Untersuchungen (...) zeigte Foucault, dass die Gesellschaft diskursiv festlegt, was als ,normal' und was als ,deviant' (als von der Norm abweichend) vorgestellt wird. “45 Dieser Aspekt ist besonders hervorzuheben, da aus dieser Bewertung in "normal“ oder „deviant" Handlungen abgeleitet werden. Aus der Einordnung eines Sachverhaltes als „erwünscht“ oder „nicht erwünscht“ resultiert demnach eine nicht unerhebliche Macht zur Beeinflussung bzw. Veränderung der Gesellschaft. Hier wird deutlich, welch wichtige Rolle gerade die nicht explizit genannten Werte, sondern die „unterschwellig“ im Text versteckten Werte spielen.

In diesem Abschnitt wurden die Schwierigkeiten einer auf Objektivität zielenden Geschichtswissenschaft diskutiert und gezeigt, wie sich dieses Thema durch die gesamte Geschichte der historischen Wissenschaften zieht. Meines Erachtens verlangt wissenschaftliche Akkuratesse eine gewisse Leidenschaft auf Seiten des Forschenden, die einer möglichst objektiven Betrachtung jedoch nicht im Wege steht. Vielmehr dürfte ein leidenschaftlicher Forscher bestrebt sein alle denkbaren Aspekte bei der Kontextbetrachtung zu berücksichtigen, inklusive des eigenen (kulturellen) Horizonts und seines Einflusses.

Eine völlige Wertfreiheit ist meiner Meinung nach jedoch unmöglich, da der Forscher seine kulturelle Horizontbedingtheit (vgl. Gadamer) nicht einfach ausblenden kann bzw. als erkennendes Subjekt zwangsläufig selbst Subjektivität in den Forschungsprozess einbringt (vgl. Schaff). Eine möglichst genaue Trennung von Fakten und Wertungen ist anzustreben, denn, und hier ist Weber und Sombart zuzustimmen, Wissenschaft kann nicht zu einem Werturteil führen. Wissenschaftliche Erkenntnisse $\mathrm{zu}$ bewerten um z.B. Handlungsempfehlungen für die Politik abzuleiten, ist meiner Meinung nach durchaus sinnvoll, jedoch muss diese Bewertung von der rein wissenschaftlichen Beschreibung getrennt werden bzw. die Trennung muss für den Leser erkennbar sein. In diesem Zusammenhang ist nochmals auf die Arbeit von Derrida und Foucault hinzuweisen, die zu der Ansicht gelangten, dass Sprache die Realität nicht nur abbildet, sondern auch formt. Wertende Sprache hat demnach Einfluss auf die Realität. Meines Erachtens trifft dies zu, denn es macht einen Unterschied

\footnotetext{
44 Jordan, S. 189.

${ }^{45}$ Ebenda, S. 189.
} 
für die Interpretation eines Textes durch den Leser, mit welchen Worten ein Phänomen beschrieben wird. Gerade diese nicht explizit benannten Wertungen sind einflussreich und damit interessant, aber fließen, wie ich vermute, oftmals unbewusst in den Text ein. Das Problem besteht nun darin, die in einem Text enthaltenen Werte, insbesondere die impliziten, zu identifizieren, was zur Fragestellung und zum Aufbau dieser Arbeit führt.

\subsection{Aufbau und Ziel dieser Arbeit}

Obwohl sich umweltgeschichtliche Forschung mit den Wechselwirkungen von Gesellschaft und Natur befasst, also einer Bewertung von wechselseitigen Einflüssen, scheinen die Werte, die der jeweiligen Beurteilung zugrunde liegen bzw. deren Ermittlung bislang nicht ausreichend erforscht zu sein. ${ }^{46}$ Radkau merkt zu dieser Forschungslücke an „Vermutlich meidet man dieses Problem, weil man dahinter ein unlösbares Dilemma wittert.“47 Dabei beeinflussen Werthaltungen bzw. die daraus resultierenden Werturteile die Forschungsarbeit, gerade auch die impliziten. „Prozeßanalysen (...) verweisen auf Auswirkungen umweltbezogener Werthaltungen auf Entscheidungen und Handlungen auch dann, wenn jene nicht bewußt werden. Implizit bleibende Werthaltungen beeinflussen Informationssuchund Verarbeitungsprozesse.“48

Aus der fehlenden expliziten Darstellung, welche Werte den Beurteilungen zugrunde liegen, folgt die Frage, welche Werte in umweltgeschichtlichen Texten eigentlich dominieren und wie diese zu ermitteln sind. Die Identifikation dieser Werte, insbesondere der impliziten, erfolgt über Indikatoren. Der Begriff Umweltschaden ist solch ein Indikator, da diesem Begriff eine Bewertung zugrunde liegt. „(...) we recognize environmental problems through a variety of health, survival, moral, empathetic, aesthetic, political, economic and cultural interests. Those interests, grounded in individual, collective, historical, cross-cultural, and visionary experience, are socially constructed (...) and socially interpreted. (...) Hence, the use of the term ,environmental problems‘ presupposes choices that we make about significant

\footnotetext{
${ }^{46}$ Die Frage nach den in einem Text enthaltenen bzw. dem Text zugrundeliegenden Werten ist nicht nur eine der umweltgeschichtlichen Forschung, sondern eine generelle. Daher liegt es in der Natur der Sache, dass sich die verschiedensten Disziplinen dieser Thematik annehmen. An dieser Stelle kann nur ein kurzer Anriss dieses Forschungsfeldes gegeben werden. Die Textlinguistik, deren Inhalt satzübergreifende Strukturen eines Textes sind, befasst sich neben der Textdefinition und -klassifikation auch mit der Textanalyse. Allerdings scheint die Analyse hinsichtlich zugrundeliegender Wertvorstellungen eher in das Feld der sozialwissenschaftlichen Diskursanalyse zu fallen, die sich u. a. mit sprachlich-rhetorischen Mitteln und inhaltlich-ideologischen Aspekten eines Textes beschäftigt. Auch die Textinterpretation hat die Aufschlüsselung von im Text vorhandenen Bedeutungen (auch solchen, die der Autor nicht intendierte bzw. unbewusst verwendete) zum Ziel, wird jedoch hauptsächlich in der Literaturwissenschaft angewendet. Am weitesten spezialisiert hat sich anscheinend die Hermeneutik, die sich auch mit speziellen (methodischen) Interpretationsaspekten einzelner Disziplinen, wie der Geschichtswissenschaft, auseinandersetzt. Aber wie in Kapitel 1.1 ausgeführt, gab und gibt es viele Ansätze zur Analyse von Werten aus den unterschiedlichsten Bereichen.

47 Joachim Radkau, Natur und Macht: Eine Weltgeschichte der Umwelt, München, 2000b, S. 33.

48 Lenelis Kruse/C.-F Graumann/E. -D Lantermann, Ökologische Psychologie: Ein Handbuch in Schlüsselbegriffen, München, 1990, S. 636.
} 
experiences and moral principles." 49 Durch eine Analyse des zugrunde liegenden Ereignisses sowie des zugehörigen Kontextes, in welchem dieser Begriff verwendet wird, lässt sich also auf die zugrunde liegenden Werte schließen.

Ein kleiner Textausschnitt verdeutlicht das unterschiedliche Verständnis von Umweltschäden: „Scheinbar am Rande liegende Naturerscheinungen konnten Warnsignale für Umweltschäden sein. (...).“50 Die angesprochene „Naturerscheinung“ ist die deutlich gestiegene Brüchigkeit von Vogeleiern im Jahr 1966 im Vergleich zu 1904, die zu Brutverlusten führte. Dieses Phänomen selbst wird aber nicht als Umweltschaden betrachtet, sondern nur als ein Hinweis darauf. Die Verwendung des Begriffs Umweltschaden erlaubt im Kontext daher einen Rückschluss auf die dem Text zugrunde liegenden Werte.

Werte beeinflussen die Wahrnehmung der Umwelt. Es scheint nicht ausreichend untersucht zu sein, wie die Werte-Problematik in umweltgeschichtlichen Texten Berücksichtigung findet. Die Kenntnis dieser Werte könnte eine neue Sicht auf die Veränderungen der Umwelt ermöglichen, da die Beschreibung der wechselseitigen Einflüsse zwischen Gesellschaft und Natur um die impliziten Werte bereinigt werden könnte. Zudem wäre es auch möglich, Phänomene bewusst unter verschiedenen Werteblickwinkeln zu beschreiben. Daraus könnte sich eine neue Vorgehensweise in der umwelthistorischen Forschung ergeben.

Diese Dissertation soll einen klärenden Beitrag leisten zu der Diskussion, welche Wertvorstellungen in umweltgeschichtlichen Texten dominieren. Dafür soll umwelthistorisches Textmaterial hinsichtlich des Verständnisses von Umweltschäden untersucht werden. Das Textmaterial soll von regionalen über universale Kontexte und von ephemeren Ereignissen bis zu langfristigen Abläufen reichen. Das Ziel der Untersuchung ist eine deskriptive Aussage zu diesen Werten, wobei die Analyse der Texte der entdeckenden Forschungslogik folgt.

Aus den vorgestellten Aspekten der Rekonstruktion von Wertvorstellungen ergibt sich für die geplante Arbeit zudem die Notwendigkeit von Abgrenzungen. Nicht Inhalt dieser Arbeit ist die Frage, warum diese Werte zugrunde gelegt und wie sie ermittelt wurden. Auch die Frage, woher die zugrundeliegenden Werte stammen, bzw. wessen Werte es sind, soll nicht behandelt werden. Im Rahmen umwelthistorischer Forschungen ist es denkbar, dass Werte verschiedener Gruppen einfließen: Bereits bei der Auswahl der umweltgeschichtlich zu bearbeitenden Themen wirken Wertvorstellungen. Welche Themen werden als wichtig erachtet, bzw. sind es wert, bearbeitet zu werden? Hier können sowohl persönliche Werte

\footnotetext{
${ }^{49}$ E. A. R. Bird, The social construction of Nature: Theoretical approaches to the history of Environmental Problems, in: Environmental Review 11 (1987) 4, S. 255-264, hier S. 260-261.

${ }^{50}$ Gottfried Zirnstein (Hrsg.), Ökologie und Umwelt in der Geschichte, Marburg, 1994.
} 
des Forschers als auch Werte der akademischen Disziplin(en) sowie heutige gesellschaftliche Werte die Themenwahl beeinflussen. Des Weiteren kann das persönliche Wertesystem des Forschers auch während der Bearbeitung Auswirkungen auf die Art der Bearbeitung haben. Gleiches gilt für die heute generalisierten Werte einer bestimmten Gesellschaft, unter deren Aspekten die Bearbeitung des Themas erfolgt. Schließlich sind noch die Werte der damals lebenden Menschen bzw. Gesellschaften zu nennen, die die Wahrnehmung ihrer Umwelt beeinflussten und - nicht zuletzt - das Wertesystem der Quellenautoren.

Im Gegensatz zu quantitativen Methoden mit standardisierten Kriterien der Reliabilität, Validität, Repräsentativität und Objektivität ist die Frage der Gütekriterien in der qualitativen Forschung nicht pauschal zu beantworten. „Eine identische Replikation einer Untersuchung ist schon allein aufgrund der begrenzten Standardisierbarkeit des Vorgehens in der qualitativen Forschung unmöglich. Angemessen für qualitatives Vorgehen ist der Anspruch auf Herstellung von intersubjektiver Nachvollziehbarkeit des Forschungsprozesses." ${ }^{\text {"Die }}$ möglichst exakte Dokumentation des Forschungsprozesses wird daher angestrebt. Die Auswahl der Analysemethode sowie die Beschreibung der Operationalisierung für die Durchführung der Untersuchung werden aus diesem Grund detailliert dargestellt. Nichtsdestotrotz orientiert sich die qualitative Analyse auch an den o.g. Gütekriterien. Bezüglich der Repräsentativität ist anzumerken, dass hier nur ein begrenzter Anspruch auf Generalisierung der Ergebnisse bestehen kann. ${ }^{52}$ Dies ist hauptsächlich in der nicht eindeutig zu definierenden Grundgesamtheit begründet, aber auch im qualitativen Untersuchungsansatz. Durch Stichprobenumfang und die Art der Stichprobenziehung sollte es jedoch möglich sein, eine Stichprobenzusammensetzung zu erzeugen, die trotzdem aussagekräftige Ergebnisse liefern kann.

Während der Literaturrecherche für diese Arbeit wurde schnell klar, dass deutschsprachige Beiträge zur Umweltgeschichte nur einen kleinen Teil der verfügbaren Texte ausmachen. ${ }^{53}$ Einen weit größeren Fundus bilden Abhandlungen in englischer Sprache und das nicht nur im englischsprachigen Raum. Vielmehr scheint es so, dass auch Forscher an Hochschulen im deutschsprachigen Raum ihre Ergebnisse gelegentlich auf Englisch publizieren ${ }^{54}$. Die

\footnotetext{
${ }^{51}$ Ines Steinke, Gütekriterien qualitativer Forschung, in: Uwe Flick/Ernst von Kardorff/Ines Steinke (Hrsg.), Qualitative Forschung. Ein Handbuch, Reinbek bei Hamburg 2009, S. 319-331, hier S. 324.

52 „Während in der quantitativen Sozialforschung die Bildung einer Stichprobe auf, ein verkleinertes Abbild der empirischen vorfindbaren Fälle' abzielt, soll die Stichprobenbildung in der qualitativen Sozialforschung ,ein Abbild der theoretisch relevanten Kategorien darstellen."“ Gabriele Rosenthal, Interpretative Sozialforschung: Eine Einführung, Weinheim, 2005, S. 85.

${ }^{53}$ Der größere Fundus an englischen Texten erklärt sich wahrscheinlich zum größten Teil dadurch, dass die Disziplin Umweltgeschichte ihren Anfang in den USA nahm, wo 1977 die ASEH (American Society for Environmental History) gegründet wurde und so ein erheblicher zeitlicher Vorsprung entstand. Die Gründung des europäischen Pendants folgte erst 1999 (ESEH, European Society for Environmental History).

${ }^{54}$ Dies dürfte der Tatsache geschuldet sein, dass die relevanten Zeitschriften zumeist in Englisch erscheinen.
} 
vorliegende Arbeit erhebt aufgrund der Stichprobengröße nur begrenzten Anspruch auf Generalisierung. Um aber trotzdem einen aussagekräftigen Querschnitt der Werte in umwelthistorischen Arbeiten zu zeigen, ist es sinnvoll, auch englischsprachige Texte einzubeziehen. Dies geschieht durch die Suche nach Zeitschriftenartikeln in den beiden Zeitschriften „Environment and History“ sowie „Environmental History“, da kaum umwelthistorische Zeitschriften in deutscher Sprache zu finden sind, aber Publikationen dieses Formats für die Fragestellung dieser Arbeit sehr gut geeignet sind (siehe auch Abschnitt 3.2.3 zum Ablauf der Stichprobenziehung).

Die vorliegende Arbeit baut sich im Weiteren wie folgt auf: Kapitel zwei beschäftigt sich zunächst mit der Disziplin Umweltgeschichte um dann Werte in der Umweltgeschichte näher zu betrachten. Im Kapitel drei folgt die Erörterung der Methodik für die Untersuchung. Nach Durchführung der Textanalyse erfolgt in Kapitel vier die Auswertung der gewonnenen Daten. In Kapitel fünf werden dann die dominierenden Werte ermittelt und anschließend einzeln betrachtet. Die Zusammenfassung der Arbeit erfolgt in Kapitel sechs; hier werden auch die Ergebnisse zusammengefasst und diskutiert. 


\section{Umweltgeschichte und Werte}

\subsection{Umweltgeschichte}

\subsubsection{Entstehung der Disziplin Umweltgeschichte}

Welche Phänomene von Historikern wahrgenommen werden bzw. welche einer Untersuchung für würdig befunden werden, hängt auch davon ab, was zur jeweiligen Zeit von allgemeinem Interesse war. Waren es früher nur Ereignisse mit politischer Relevanz, so war es im 19./20. Jahrhundert u. a. die Industrialisierung und ihre Folgen. Den Beginn der Umweltbewegung $^{55}$ Mitte des 20. Jahrhunderts könnte man somit die Wiege der Umweltgeschichte nennen, da nun Umweltthemen in den Fokus traten. Zumindest bezüglich der Disziplin Umweltgeschichte, wie wir sie heute kennen, denn „Punktuelle und sporadische Naturschäden hat es seit der Antike immer wieder gegeben; so klagte man bereits zur Zeit Platons über abgeholzte Berghänge und die Folgen für das lokale Klima. ${ }^{“ 56}$ Die Themen der Umweltgeschichte sind also keine Neuen. Aber durch die modernen Möglichkeiten des Menschen, die Umwelt zu verändern, werden die Fragestellungen heute drängender. „Die Menschen, oder wenigstens ein Teil von ihnen, haben aber solche Mittel und Einflussmöglichkeiten erworben, dass sie bis zu einem gewissen Grade dieser Naturordnung entkommen und so stark auf die natürliche Umwelt einwirken konnten, dass sie großenteils eine andere wurde. (...) Aber viele Menschen sind heute wie früher von ihren Trieben beherrscht und fragen zu wenig nach den Folgen ihres Tuns auf der Erdoberfläche. “57

Die Umweltgeschichte ist eine vergleichsweise junge Disziplin, die sich in den 1970erJahren $^{58}$ herausgebildet hat. Ihr Thema ist die Untersuchung von Wechselwirkungen zwischen Gesellschaft und Natur. Daraus ergibt sich ein transdisziplinärer ${ }^{59}$ Arbeitsansatz, der Methoden und Erkenntnisse verschiedener Disziplinen miteinander verbindet. Im Idealfall nehmen alle diese Disziplinen und ihre jeweiligen Methoden und Sichtweisen Einfluss und befähigen so den Forscher, seinen Untersuchungsgegenstand in vielfältiger Art und Weise zu betrachten und zu interpretieren. Diese Verzahnung unterschiedlicher Disziplinen stellt

\footnotetext{
${ }^{55}$ Auch über den Beginn der Umweltbewegung, sowohl zeitlich als auch räumlich, kann man sicher diskutieren: „Doch tatsächlich ist der abendländische Naturschutzgedanke mehr als 200 Jahre alt und seine Wurzeln liegen in den Tropen." Richard H. Grove, Die Anfänge des Umweltbewußtseins, in: Ernst Ulrich von Weizsäcker (Hrsg.), Mensch, Umwelt, Wirtschaft, Heidelberg (u. a.) 1995, S. 18-23, hier S. 18.

${ }^{56}$ Konrad Ott, Umweltethik zur Einführung, Hamburg, 2010, S. 25.

57 Zirnstein, Zirnstein 1994, S. 14.

${ }^{58}$ Für Engels liegt es nahe, aufgrund der politischen Erfolge der Umweltbewegung in den 1970er Jahren diese Zeit als „ökologische Wende“ zu bezeichnen. Vgl. Jens Ivo Engels, Umweltgeschichte als Zeitgeschichte, in: Aus Politik und Zeitgeschichte 13 (2006) 56, S. 32-38, hier S. 35.

59 Ob „transdisziplinär“ oder „interdisziplinär“ das Arbeitsfeld der Umweltgeschichte zutreffender beschreibt, darüber kann gewiss vortrefflich gestritten werden. Der Punkt ist, dass Umweltgeschichte nicht nur traditionell historische Fragestellungen mit methodischer Hilfe anderer Disziplinen bearbeitet, sondern darüber hinaus entstammen die Fragestellungen selbst verschiedenen Disziplinen.
} 
zugleich auch eine Schwierigkeit dar. So unterscheiden sich die Methoden und Forschungsansätze der Naturwissenschaften oftmals von denen der Geisteswissenschaften. Dieser Aspekt wird in Abschnitt 2.1.3 noch näher beleuchtet. Genau an dieser Schnittstelle ist nun die Umweltgeschichte angesiedelt.

Die ersten umweltgeschichtlichen Forschungsarbeiten wurden in den USA veröffentlicht. 1958 erschien Samuel P. Hays' "Conservation and the Gospel of Efficiency: The Progressive Conservation Movement, 1890-1920" (University of Pittsburgh), das eigentlich von amerikanischer Politikgeschichte handelt, aber als eine der ersten Arbeiten umweltgeschichtliche Aspekte enthält. ${ }^{60}$ Das mit Abstand bekannteste Werk, das sowohl für die Umweltbewegung als auch für die Umweltgeschichte einen enormen Entwicklungsschub bedeutete, ist das 1962 von Rachel Carson publizierte Buch „Silent Spring“. 611967 folgte eine Veröffentlichung des Historikers Lynn White jr., die von Schenk als „früher Schlüsseltext der Umweltgeschichtsschreibung“ bezeichnet wird: „The historical roots of our ecological crisis" thematisiert "die angeblich naturausbeutende und -zerstörende westliche Mentalität als Folge einer anthropozentrischen Ausrichtung des (mittelalterlichen) Christentums."62 Ebenfalls 1967 veröffentlicht wurde „Wilderness and the American Mind“ von Roderick Nash $^{63}$, das mit "Wilderness" ein Thema bzw. ein Naturkonzept behandelt, das bis heute diskutiert wird. Es folgte 1977 die Gründung der American Society of Environmental History (ASEH). In Europa wurde eine entsprechende Vereinigung erst 1999 gegründet: die European Society of Environmental History (ESEH).

Als Einstimmung auf das eigentliche Thema dieser Arbeit - Werte in der Umweltgeschichte soll diese kurze Skizze der Anfänge im umwelthistorischen Bereich genügen. Eine umfangreiche Ausarbeitung zur Entwicklung der Disziplin hat McNeill verfasst. ${ }^{64}$ In seinem Aufsatz zeichnet er nicht nur die Anfänge der Umweltgeschichte nach, sondern beschreibt auch den Status der umwelthistorischen Forschungen nach Ländern bzw. Kontinenten. Auch in dem mittlerweile zum Standardwerk gewordenen Buch „Umweltgeschichte“ von Winiwarter

\footnotetext{
${ }^{60}$ Wie Hays selbst schreibt, ergab es sich, dass sich sein Interesse an umweltgeschichtlichen Themen mit einem Forschungsstipendium zu Roosevelt verbinden ließ, sodass er seine Dissertation zu o.g. Thema verfasste und dann als Buch veröffentlichte. Vgl. Hays, Hays 1998, S. xiv.

61 „Our Synthetic Environment“ von Murray Bookchin (veröffentlicht unter dem Pseudonym „Lewis Herber") erschien 1962 etwas früher als „Silent Spring“, wurde aber nicht annähernd so populär, wenngleich es auch Umweltschutz zum Thema hat.

${ }^{62}$ Gerrit Jasper Schenk, Der Mensch zwischen Natur und Kultur. Auf der Suche nach der Umweltgeschichtsschreibung in der deutschsprachigen Mediävistik - eine Skizze, in: François Duceppe-Lamarre (Hrsg.), Umwelt und Herrschaft in der Geschichte, München 2008, S. 27-51, hier S. 43-44.

${ }^{63}$ Neben den o.g. Hays, Carson, White und Nash, die am Beginn der umweltgeschichtlichen Forschung standen, sind u. a. Leopold, Marsh, McNeill, Muir, J.F. Richards, Simmons und Thoreau als Autoren im englischsprachigen Raum zu nennen.

${ }^{64} \mathrm{Vgl}$. J. R. McNeill, Observations on the nature and culture of environmental history, in: History and Theory 42 (2003) 4, S. 5-43.
} 
und Knoll gibt es einen Abschnitt zur Entwicklung der Disziplin. ${ }^{65}$ Eine äußerst strukturierte und umfangreiche Darstellung hat Uekötter in seinem Werk „Umweltgeschichte im 19. und 20. Jahrhundert“ ${ }^{66}$ vorgelegt.

Werfen wir nun einen Blick auf die gängigen Definitionen zur Umweltgeschichte. „Umweltgeschichte erscheint im weitesten Sinne als Geschichte des menschlichen Handelns zur Nutzung und Umgestaltung der Welt, der diesbezüglichen Ideologien und Motivationen, der Wahrnehmung der Veränderungen und der Reaktionen darauf.“67 „Umweltgeschichte beschäftigt sich mit der Rekonstruktion von Umweltbedingungen in der Vergangenheit sowie mit der Rekonstruktion von deren Wahrnehmung und Interpretation durch die damals lebenden Menschen'. Diese Definition von Umweltgeschichte geht auf Bernd Herrmann und Rolf Peter Sieferle zurück.“68 Beide Definitionen zeigen, dass sich Umweltgeschichte als Ganzes zwar recht gut umschreiben lässt, jedoch gleichzeitig die schier unglaubliche Spannweite der möglichen Themen. Entsprechend schwierig scheint die Einordnung der Umweltgeschichte in die bestehende Wissenschaftslandschaft. „Verbreitet ist demnach die Verortung der Umweltgeschichte im Bereich der Kultur- und Mentalitätsgeschichte, der historischen Anthropologie, Verhaltens- oder Wahrnehmungsgeschichte. Kaum weniger häufig wird sie der Sozial- und Wirtschafts-, der Alltags- und Sachkulturgeschichte zugeordnet. Doch sie kann auch als ein Aspekt spezieller Themenfelder wie der Stadtgeschichte, der Geschichte des hochmittelalterlichen Landesausbaus, der Energiegeschichte, der Geschichte des Mensch-Tier-Verhältnisses, der Geschichte der Entsorgung und der Medizingeschichte verstanden werden." ${ }^{69}$ Hier deutet sich bereits an, welche Unzahl an Begriffsbestimmungen und Abgrenzungen erforderlich sind. „Zunächst die Feststellung, dass das Forschungsfeld eher durch eine umweltgeschichtliche Fragestellung als durch Gegenstand, Quellen und Methoden konstituiert wird. (...) Folgerichtig rangen bisher und ringen auch heute noch (...) viele, die sich erklärtermaßen mit Umweltgeschichte beschäftigen, um Begriffsklärung, Standortbestimmungen, Abgrenzungen, Definitionen und Beschreibungen dessen, was sie tun - zugleich Merkmal reflektierender Wissenschaft die Kennzeichen einer (sub-)disziplinären Formierungsphase.“70 ${ }^{70}$ Diese Schwierigkeiten der

\footnotetext{
${ }^{65}$ Vgl. Winiwarter et al., Winiwarter et al. 2007, S. 30-42.

${ }^{66}$ Frank Uekötter, Umweltgeschichte im 19. und 20. Jahrhundert, München, 2007.

${ }^{67}$ Herrmann Fuchsloch, Methodenfragen der Umweltgeschichte, in: Günter Bayerl (Hrsg.), Umweltgeschichte Methoden, Themen, Potentiale, Münster 1996, S. 1-12, hier S. 6.

${ }^{68}$ Lars Kreye/Markus Schwarzer, Verständnis und Institutionalisierung der Umweltgeschichte, in: Patrick Masius /Ole Sparenberg/Jana Sprenger (Hrsg.), Umweltgeschichte und Umweltzukunft. Zur gesellschaftlichen Relevanz einer jungen Disziplin, Göttingen 2009, S. 3-11, hier S. 4-5 und Vorlesung Prof. Herrmann in Göttingen am 22.10.2010.

${ }^{69}$ Schenk, Schenk 2008, S. 36-37.

${ }^{70}$ Ebenda, S. 38.
} 
Umweltgeschichte kann man gut an der Entwicklung der Disziplin in Deutschland beobachten.

„Angeregt von amerikanischen Veröffentlichungen hat Wolfgang Zorn 1978 in der Bundesrepublik Deutschland die Frage nach der Stellung des Faches Geschichte, insbesondere der Sozial- und Wirtschaftsgeschichte, zur Umweltkrise der Gegenwart in einem bis heute grundlegenden Beitrag aufgenommen. Er zeigt dabei in einem weiten Spektrum Umweltschäden als zumeist langfristige Folge ertragswirtschaftlichen Handelns des Menschen auf, die sich von frühen Gesellschaften über bäuerlich-gewerblich organisierte bis zur industriellen der Gegenwart hin verschärften (...).“11 1981 bescheinigt Troitzsch der Umweltgeschichte einen kaum nennenswerten Forschungsstand: „Zunächst folgt ein Überblick über den Forschungsstand, der allerdings relativ knapp ausfallen kann, da man bislang von einer historischen Umweltforschung mit klar abgesteckten Forschungsbereichen und Forschungszielen kaum sprechen kann. Das Häuflein derer, die sich, vorwiegend nebenher, damit bislang beschäftigt haben (...) ist zahlenmäßig äußerst klein, rekrutiert sich aus den unterschiedlichsten Wissenschaftsdisziplinen und weist kaum personelle, geschweige denn institutionelle Kontakte auf." ${ }^{72}$ Allerdings räumt Troitzsch ein, dass es durchaus mehr Untersuchungen zur Umweltproblematik gibt als zunächst gedacht, da „der größte Teil solcher Untersuchungen in medizinischen, technischen und naturwissenschaftlichen Zeitschriften erschienen sind, die dem Historiker kaum bekannt und zugänglich sind."73 Hierbei muss auf den Zeitpunkt dieser Aussage hingewiesen werden: 1981 begann gerade die Umstellung des ursprünglichen Arpanet auf das Internetprotokoll. Das heute bekannte World Wide Web wurde erst 1989 entwickelt und verbreitete sich ab etwa 1993. Die Aussage von Troitzsch macht deutlich, dass das, was heute als selbstverständlich betrachtet wird (leicht verfügbare Daten), damals ein großes Problem war; insbesondere für interdisziplinär Forschende.

Die knapp 20 Jahre später verfasste Beschreibung von Radkau zeigt eine nicht gerade positive Entwicklung: „Seit etwa zwanzig Jahren finden in der Bundesrepublik Tagungen über Umweltgeschichte statt, und die Literatur dazu hat ein ansehnliches Volumen erreicht; aber bis heute gibt es kaum eine einzige ausdrücklich der Umweltgeschichte gewidmete Hochschulstelle. Tagungen über ,Environmental History' in den USA sind jährlich stattfindende Massenveranstaltungen großen Stils in Nobelhotels; entsprechende Tagungen in Europa halten sich in aller Regel in einem sehr viel bescheideneren Rahmen, - und das, obwohl es für Umwelthistoriker in der Alten Welt unendlich viel mehr zu entdecken gäbe als

\footnotetext{
${ }^{71}$ Paul Leidinger, Von der historischen Umweltforschung zur Historischen Ökologie. Ein Literaturbericht (Teil 1), in: Hermann Behrens/Regine Auster (Hrsg.), Umweltgeschichte Wissenschaft und Praxis. Umweltgeschichte und Umweltzukunft II, Marburg 1994, S. 11-39, hier S. 16.

72 Ulrich Troitzsch, Historische Umweltforschung: Einleitende Bemerkungen über Forschungsstand und Forschungsaufgaben, in: Technikgeschichte 48 (1981) 3, S. 177-190, hier S. 179.

${ }^{73}$ Ebenda, S. 180.
} 
in der Neuen und obwohl die deutsche Umweltbewegung eine internationale Spitzenstellung hat oder zumindest gehabt hat." ${ }^{74}$ Demgegenüber steht eine positive Bewertung zur Entwicklung der Disziplin Umweltgeschichte von Uekötter: „Das vielleicht markanteste Merkmal der deutschen Umweltgeschichtsforschung ist ihre enorme Dynamik. (...) Vielleicht war der lange Zeit niedrige Institutionalisierungsgrad in mancher Beziehung sogar ein Vorteil: Bis heute ist die deutsche Umweltgeschichte kaum von rivalisierenden ,Schulen' geprägt, stattdessen bleibt eine Offenheit auch gegenüber Außenseitern erhalten, denen die Forschung immer wieder überraschende Impulse verdankte.." ${ }^{75}$ Dem stimmt McNeill zu: „Environmental history can claim with justice to have become one of the most vital sub-fields within the historical discipline, at least in a handful of countries." ${ }^{76}$

Brüggemeier stellt fest, dass die Disziplin Umweltgeschichte in Deutschland zwar von einem deutlichen Aufschwung gekennzeichnet ist, aber im Gesamtbild der Geschichtswissenschaften kaum eine Rolle spielt. So findet Umweltgeschichte in Einführungen zu Geschichtswissenschaft kaum Berücksichtigung. ${ }^{77}$ Dem stimmt McNeill zwar zu, weist aber zugleich darauf hin, dass sich Qualität und Bandbreite der umweltgeschichtlichen Arbeiten in Nordeuropa durchaus mit anderen Regionen, inklusive der USA, messen können. ${ }^{78}$ Kalt nennt die mangelnde Definition als Grund für die untergeordnete Rolle der Umweltgeschichte: „Das Dilemma ist offensichtlich: Umweltgeschichte lässt sich nicht klar definieren. (...) Doch die mangelnde theoretische Durchdringung des Untersuchungsgegenstandes ist (...) ein Grund, weshalb Umweltgeschichte sich bislang nicht so recht durchzusetzen vermochte.“" ${ }^{79}$ McNeill sieht in dieser Unschärfe jedoch auch Vorteile: „The borders among disciplines, and among genres within a discipline, are never sharp and watertight. In the case of environmental history, they are especially fuzzy and porous. In that lies some of the appeal of environmental history." ${ }^{30}$ Des Weiteren sieht er durchaus einen wachsenden Einfluss der Umweltgeschichte: „Despite the awkward challenges it can pose to historians, as it marches along it is certainly leaving a growing footprint upon the discipline of history generally." ${ }^{\text {"81 }}$

\footnotetext{
${ }^{74}$ Joachim Radkau, Erfahrungen und Grübeleien beim Schreiben an einer Welt-Umweltgeschichte, in: Universität Bielefeld, Informations- und Pressestelle (Hrsg.), Forschung an der Universität Bielefeld, Bielefeld 2000, S. 4249, hier S. 43.

${ }^{75}$ Uekötter, Uekötter 2007, S. 88.

${ }^{76}$ McNeill, McNeill 2003, S. 42.

77 Vgl. Franz-Josef Brüggemeier, Umweltgeschichte - Erfahrungen, Ergebnisse, Erwartungen, in: Archiv für Sozialgeschichte 43 (2003), S. 1-18, hier S. 1.

${ }^{78}$ Vgl. McNeill, McNeill 2003, S. 19.

${ }^{79}$ Monika Kalt, Umweltgeschichte - Revisited, in: Traverse: Zeitschrift für Geschichte 4 (1997) 2, S. 13-30, hier S. 20.

${ }^{80}$ McNeill, McNeill 2003, S. 9.

${ }^{81}$ Ebenda, S. 11.
} 
Das, was die Umweltgeschichte als Forschungsfeld so interessant macht, nämlich die ungeheure Bandbreite an Themen und beteiligten Disziplinen, ist zugleich ein Schwachpunkt. Radkau stellt hierzu fest: „Was in der historischen Umweltforschung gegenwärtig am meisten fehlt, ist der Zusammenhang, der größere Kontext. Weltweit und fächerübergreifend gesehen, gibt es heute eine geradezu überwältigende Fülle umwelthistorischer Forschungsansätze; aber sie sind auf verschiedene Disziplinen und Unterdisziplinen, Länder und Regionen verstreut und wissen großenteils nichts voneinander. (...) bei alledem fehlt es in erstaunlichem Maße an Wechselbeziehungen. Die bisherige Art des Spezialistentums ist dazu offenbar nicht fähig.“82 Der hier angesprochene Punkt des „Spezialistentums“ wird in Abschnitt 2.1.3 ausführlicher diskutiert. Auch Hays bemängelte schon früh, dass es der Umweltgeschichte noch nicht gelungen ist, ihre vielen Einzelstücke zusammenzusetzen und fasst zusammen: „Unless we inform our research in environmental history more broadly its meaning for the larger frame of historical writing will remain obscure." 83

Wie aber kann sich die Umweltgeschichte als Disziplin weiterentwickeln? Radkau meint, dass sich die Umweltforschung zunächst einmal die offenen Fragen eingestehen und diese dann auch präzisieren muss. ${ }^{84}$ Eine der wichtigsten Fragen dürfte die nach der Ordnung des Forschungsfeldes sein um die Forschungsansätze in einen Zusammenhang zu bringen. Abbildung 1 zeigt vier unterschiedliche Ansätze zur Strukturierung der Umweltgeschichte. Radkau listet fünf Gedankenansätze auf, an denen sich eine Weltumweltgeschichte orientieren sollte. ${ }^{85}$ Die Strukturierung von Radkau bietet eine sinnvolle Aufteilung der Themen, auch da die Titel Platz zur Interpretation lassen, wenngleich er z.T. sehr unterschiedliche Kategorien vorschlägt. Die Kategorie „Methode der Konstruktion von Umweltproblemen“ sollte meiner Meinung nach weiter gefasst werden, z.B. als „Methode zur Konstruktion von Umweltwahrnehmung“, wobei Umweltprobleme eine Unterkategorie hiervon bilden könnten. Von Borries gliedert in sechs Kategorien ${ }^{86}$, wobei die Trennung in anthropozentrische (fünf Kategorien) und nicht-anthropozentrische (eine Kategorie) Themen auffällt. An von Borries' Aufteilung ist zunächst zu kritisieren, dass es eine nichtanthropozentrische Kategorie gibt. In Kapitel 2.1.1 wurden Definitionen der Disziplin Umweltgeschichte vorgestellt, wobei alle Definitionen einen Bezug zum Menschen enthalten. Dieser anthropozentrische Ansatz der Umweltgeschichte wird in Abschnitt 2.2.5 dieser Arbeit weiter diskutiert, wobei ich zu dem Schluss gelange, dass die Themen der Umweltgeschichte immer einen anthropozentrischen Ansatz enthalten. Daher ist die von von Borries

\footnotetext{
${ }^{82}$ Radkau, Radkau 2000, S. 42.

${ }^{83}$ Hays, Hays 1998, S. 332.

${ }^{84} \mathrm{Vgl}$. Joachim Radkau, Nachdenken über Umweltgeschichte, in: Wolfram Siemann (Hrsg.), Umweltgeschichte. Themen und Perspektiven, München 2003, S. 165-186, hier S. 182.

${ }^{85}$ Vgl. Radkau, Radkau 2000, S. 45-48.

${ }^{86}$ Vgl. Fuchsloch, Fuchsloch 1996, S. 11.
} 
angedachte Kategorie „menschenunabhängige Naturgeschichte der Umwelt" nicht sinnvoll. Des weiteren ist die Abgrenzung zwischen den Kategorien „menschengemachte Veränderungen der Umwelt" und "menschliche Kulturerzeugnisse als Umwelt" etwas unscharf, da menschliche Kulturerzeugnisse auch Veränderung der Umwelt bewirken bzw. bewirken können. Zudem enthält die Kategorie "gesellschaftliche Handlungen zur Umweltverbesserung“ eine Wertung („Verbesserung“). Dies ist meines Erachtens problematisch, da zum einen unklar ist für wen oder was die Umwelt besser werden soll und was „besser" bedeutet, und zum anderen, da eine Kategorie „Verbesserung" eigentlich eine weitere Kategorie „Verschlechterung“ erfordert, um diese Fälle nicht auszuschließen. Insgesamt überzeugt der Vorschlag von von Borries nicht.

\begin{tabular}{|c|c|}
\hline Radkau $^{87}$ & $\begin{array}{l}\text { - Stoffkreisläufe } \\
\text { - Leitbild Koevolution Mensch-Natur } \\
\text { - Medien Wald, Wasser, etc. } \\
\text { - Methode der Konstruktion von Umweltproblemen } \\
\text { - Suche nach verborgenen Stabilisatoren der Mensch-Umwelt-Beziehung }\end{array}$ \\
\hline von Borries ${ }^{88}$ & $\begin{array}{l}\text { - menschenunabhängige Naturgeschichte der Umwelt } \\
\text { - gesellschaftliche Relevanz von Umwelt } \\
\text { - menschengemachte Veränderungen der Umwelt } \\
\text { - menschliche Wahrnehmung von Umwelt und Umweltveränderung } \\
\text { - gesellschaftliche Handlungen zur Umweltverbesserung } \\
\text { - menschliche Kulturerzeugnisse als Umwelt }\end{array}$ \\
\hline Brüggemeier ${ }^{89}$ & $\begin{array}{l}\text { - natürliche Umwelt } \\
\text { - Auseinandersetzung der Menschen mit der natürlichen Umwelt } \\
\text { - Wahrnehmung und Ideologien zur Umwelt bzw. Natur }\end{array}$ \\
\hline McNeill $^{90}$ & $\begin{array}{l}\text { - materiell } \\
\text { - kulturell/intellektuell } \\
\text { - politisch }\end{array}$ \\
\hline
\end{tabular}

\section{Abbildung 1: Ansätze zur Strukturierung der Umweltgeschichte}

Brüggemeier schlägt dagegen in Anlehnung an D. Worster die Aufteilung in drei Untersuchungsebenen vor. ${ }^{91}$ Wie auch bei von Borries gibt es bei Brüggemeier eine menschenunabhängige Kategorie, hier als „natürliche Umwelt“ bezeichnet, die meines

\footnotetext{
${ }^{87}$ Vgl. Radkau, Radkau 2000, S. 45-48.

${ }^{88} \mathrm{Vgl}$. Fuchsloch, Fuchsloch 1996, S. 11.

${ }^{89} \mathrm{Vgl}$. ebenda.

${ }^{90}$ Vgl. McNeill, McNeill 2003, S. 6.

${ }^{91} \mathrm{Vgl}$. ebenda.
} 
Erachtens nicht zur anthropozentrisch geprägten Umweltgeschichte passt. Zumindest nicht nach heutiger Definition. Eine Erweiterung der Definition von Umweltgeschichte ist grundsätzlich denkbar, jedoch steht dem meiner Meinung nach die heute schon sehr umfangreiche Fragestellung der Umweltgeschichte entgegen. Auch McNeill unterteilt Umweltgeschichte in drei Bereiche, wobei sich der materielle Bereich mit den Veränderungen der biologischen und physikalischen Umwelt befasst und damit, wie diese Veränderungen auf den Menschen wirken. ${ }^{92}$ Diese drei Bereiche lassen sich gut voneinander abgrenzen und decken gleichzeitig wichtige Kernfragen der Umweltgeschichte ab. Andererseits ist es meiner Ansicht nach fraglich, ob diese Teilung sinnvoll ist. Wenn z.B. die Forstbestandsentwicklung eines Gebiets analysiert werden soll, dürfte es zum Gesamtverständnis erforderlich sein, nicht nur die faktischen Veränderungen der Umwelt darzustellen, sondern auch den kulturellen bzw. politischen Einfluss darauf zu betrachten. Diese von McNeill vorgeschlagene Gliederung innerhalb einer Abhandlung anzuwenden scheint mir jedoch sinnvoll, da eine Abgrenzung der einzelnen Aspekte vielleicht klarere Schlussfolgerungen begünstigt.

Diese vier gedanklich zum Teil sehr unterschiedlichen Herangehensweisen zur Ordnung der umweltgeschichtlichen Arbeiten deuten die Schwierigkeiten an, die die wissenschaftliche Gemeinschaft auf dem Weg zur weiteren Institutionalisierung der Umweltgeschichte noch lösen muss. Umweltgeschichte ist ein spannendes und in Bewegung befindliches Forschungsfeld. Und gerade weil es sich etwas unkontrolliert entwickelt und ausbreitet, und viele verschiedene Disziplinen beteiligt sind, ist es umso interessanter herauszufinden, welche Werte in den Texten dominieren.

\subsubsection{Themenspektrum der Umweltgeschichte}

Nachdem wir einen kurzen Blick auf die Entstehung und den aktuellen Stand der Disziplin Umweltgeschichte geworfen haben, dürfte deutlich geworden sein, dass das Themenspektrum extrem weit gefasst ist und es, wie eben gezeigt, noch an einer fächerübergreifenden Ordnung fehlt. Nichtsdestotrotz sollen hier nun mögliche Themen der Umweltgeschichte ${ }^{93}$ beschrieben werden, um dann auf dieser Basis die Methoden dieses vielseitigen Fachgebietes zu erörtern.

\section{Rekonstruktion von Naturwahrnehmung und Handlungsregeln:}

„Um das Geflecht umweltrelevanter Diskurse, Institutionen und Akteure auf beispielsweise den Ebenen Wissenschaft, Politik, Recht und Öffentlichkeit zu untersuchen, liegt

\footnotetext{
92 Vgl. McNeill, McNeill 2003, S. 6.

${ }^{93}$ Weder erhebt die Themenauswahl Anspruch auf Vollständigkeit, noch ist die Reihenfolge hierarchisch zu interpretieren.
} 
grundsätzlich ein Schwerpunkt umwelthistorischer Forschung auf der Rekonstruktion von Wahrnehmungen und Deutungen sowie handlungsleitenden Konzepten und Praktiken."94 Sieferle hebt die Wichtigkeit von Naturwahrnehmung hervor: „Die Befassung mit kulturellen ,Wahrnehmungen' von Natur und Umwelt in der Vergangenheit ist alles andere als irrelevant." ${ }^{95}$ Interessanterweise stellt Kreye zehn Jahre später fest, „(...) dass die umwelthistorische Forschung bisher häufig eine zu starke Konzentration auf materielle Aspekte vorgenommen hat. Es wurde darauf hingewiesen, dass emotionale und ästhetische Zugangsweisen in der Auseinandersetzung mit der Umwelt nicht unberücksichtigt bleiben dürften.“96

Interaktive Prozesse zwischen Mensch und Natur analysieren und verstehen:

Hughes unterteilt die wechselseitigen Wirkungen zwischen Mensch und Natur in drei Bereiche. „Their choice of themes falls into three very broad categories:

(1) the influence of environmental factors on human history;

(2) the environmental changes caused by human actions and the many ways in which human-caused changes in the environment rebound and affect the course of change in human societies; and

(3) the history of human thought about the environment and the ways in which patterns of human attitudes have motivated actions that affect the environment."

Während der Analyse von Wechselwirkungen kann eine solche Aufteilung gewiss hilfreich sein, als grundsätzlichen Themenkategorien ist dieser Gliederung nicht zu folgen, da sowohl der Einfluss von Umweltfaktoren auf den Menschen als auch der Einfluss von menschlichen Einstellungen auf umweltrelevante Handlungen jeweils nur einen kleinen Prozessausschnitt darstellen, aber gerade das Wechselspiel zwischen Mensch und Natur von Interesse ist. Lehmkuhl sieht die Analyse der Mensch-Natur-Interaktion auch als grundsätzlich hilfreich an: „Environmental history can be of great importance to the general study of human-nature interaction by phenomenologically identifying various social, economic, and ecological processes in the past and analytically separating relevant patterns from each other. Successful pattern descriptions can identify recurring features of socio-ecological dynamics and enable enlightened guesses as to how they functioned. Detailed description of past events furthermore forces environmental historians to draw analytical distinctions and define

\footnotetext{
${ }^{94}$ Kreye et al., Kreye et al. 2009, S. 5.

${ }^{95}$ Rolf Peter Sieferle, Einleitung: Naturerfahrung und Naturkonstruktion, in: Rolf Peter Sieferle/Helga Breuninger (Hrsg.), Natur-Bilder. Wahrnehmungen von Natur und Umwelt in der Geschichte, Frankfurt/Main 1999, S. 9-18, hier S. 10-11.

${ }^{96}$ Kreye et al., Kreye et al. 2009, S. 10.

97 J. Donald Hughes, What is environmental history?, Cambridge, 2006, S. 3.
} 
criteria for the identification of environmental change. “98 Radkau betont, dass insbesondere versehentliche Auswirkungen untersucht werden sollten: "Gerade unbeabsichtigte Wirkungsketten menschlichen Handelns, bei denen sich Naturzusammenhänge bemerkbar machen, verdienen besondere Aufmerksamkeit." ${ }^{99}$

\section{Kritische Betrachtung des cultural turn im Bereich Geschichte:}

„Environmental history also critically reflects the "cultural turn' 100 in history and the humanities by asking how new cultural approaches can enrich our understanding of the interaction between nature and culture, ecology and culture, technology and culture and also economy and culture." ${ }^{101}$

\section{Das gesamte Mensch-Natur-Verhältnis (statt einzelner Probleme):}

„Wo könnten die Schwerpunkte einer Umweltgeschichte liegen? Angesichts der Reflexion aktueller Probleme verwundert der vielfach ausgeprägte Gegenwartsbezug nicht. Andererseits sind viele Arbeiten auf der Suche nach dem ,point of no return' in der Geschichte, von dem aus die Entwicklung unumkehrbar verlaufen ist. (...) Dabei sollte die Umweltgeschichte wie Joachim Radkau fordert - nicht Krisen und Probleme, sondern auch Anpassungsleistungen und damit das gesamte Mensch-Natur-Verhältnis in den Blick nehmen.“102

\section{(Gefährdete) Lebensbedingungen:}

„Das Ausmaß des Zugriffs auf die globalen Rohstoffvorräte im 20. Jahrhundert ist historisch beispiellos und insofern ein umwelthistorisch bedeutsamer Prozess ersten Ranges." 103 Etwas weiter fasst Engels: „Beim Umgang mit Natur und Umwelt handelt es sich um das Thema der (gefährdeten) Lebensbedingungen; dieses ist ein für Industriegesellschaften konstitutives Problem."104 Sieferle meint, dass sich die Umweltgeschichte mehrheitlich mit Umweltverschmutzung beschäftigt: „In der Begrifflichkeit Rolf Peter Sieferles lässt sich wohl die Mehrzahl der mediävistischen Arbeiten der letzten 30 Jahre zu Umweltthemen einem ,umwelthygienischen Ansatz' zuordnen, den er in die Nähe sozialgeschichtlicher Konzepte rückt. Mit dieser Charakterisierung meint Sieferle, dass in erster Linie retrospektiv, manchmal

\footnotetext{
${ }^{98}$ Ursula Lehmkuhl, Historicizing Nature: Time and Space in German and American Environmental History, in: Ursula Lehmkuhl/Herrmann Wellenreuther (Hrsg.), Historians and nature. Comparative approaches to environmental history, Oxford 2007, S. 17-44, hier S. 38.

${ }^{99}$ Radkau, Radkau 2003, S. 169.

${ }^{100}$ Der cultural turn markierte in den 1970er-Jahren die Abkehr von Kultur als Hochkultur der Eliten hin zu einem erweiterten Kulturverständnis, das die Alltagskultur einschließt.

101 Ursula Lehmkuhl, Introduction, in: Ursula Lehmkuhl/Herrmann Wellenreuther (Hrsg.), Historians and nature. Comparative approaches to environmental history, Oxford (u. a.) 2007, S. 1-5, hier S. 1.

102 Sylvia Hahn/Reinhold Reith, Einleitung, in: ders., Umwelt-Geschichte. Arbeitsfelder - Forschungsansätze Perspektiven, Wien, S. 7-12, hier S. 9.

${ }^{103}$ Uekötter, Uekötter 2007, S. 56.

${ }^{104}$ Engels, S. 37.
} 
auch wertend nach beeinträchtigenden Wirkungen menschlicher Kultur auf eine häufig normativ harmonisch gedachte Natur gefragt wird, die Aufmerksamkeit also dem Umgang mit - nach gegenwärtigem Verständnis - ,Umweltsünden“ der Vergangenheit gilt.“"105 Miller fasst zusammen und inkludiert auch eine abschließende Bewertung: „If environmental history has an important role to play, it is to remind us of what once was, what has been lost, and whether or not it was worth the price.“106

\section{Problemlösungen aus geschichtlicher Erkenntnis:}

Leidinger geht noch einen Schritt weiter und mahnt als Aufgabe der Umweltgeschichte nicht nur die Bewertung, sondern auch normative Schlussfolgerungen an: „Aufgabe des Faches sei es vielmehr, die Ökologieproblematik der Gegenwart an abgeschlossenen Tatsachen zu messen und die geschichtlichen Erkenntnisse für den gesellschaftlichen Dialog um Problemlösungen im Sinne humaner Grundentscheidungen nutzbar zu machen.“107

\section{Reizschwelle für Widerstand gegen Umweltschäden:}

„Er [Troitzsch A. d. V.] formuliert dazu allerdings eine Reihe von Fragestellungen, die für eine historische Ökologie von Bedeutung sind: (...) 3. Die Bestimmung der jeweiligen ,Reizschwelle', an der eine Gesellschaft Umweltbelästigungen der verschiedensten Art als so beeinträchtigend empfindet, dass sich Widerstand dagegen regt.“108

\section{Zusammenhang von Religion und Umweltschutz/-zerstörung:}

„Lynn White (1967) war eine der ersten, die einen Zusammenhang zwischen Christentum und einem ausbeuterischen Umweltverhalten hergestellt haben. Von anderen Hochreligionen - von Buddhismus, Hinduismus, Islam und Taoismus - wurde demgegenüber behauptet, sie implizierten ein harmonisches Verhältnis zur Natur und enthielten eine handlungsrelevante Umweltethik. (...) Die These der ökologischen Handlungswirksamkeit religiöser Vorstellungen beruht auf zwei Behauptungen: Erstens, religiöse Ideen beeinflussen Umweltverhalten in relevanter Weise; zweitens, die religiösen Ideen schreiben normativ ein umweltschonendes Verhalten vor." ${ }^{109}$ Helbing führt weiter aus, weshalb Religion für manche Menschen handlungsleitend ist: „Auf die religiösen Systeme greifen Akteure zurück, um Kontingenzen in Krankheit, Tod, Geburt, Naturkatastrophen, Missernten etc. zu erklären; sie bieten ein

\footnotetext{
${ }^{105}$ Schenk, Schenk 2008, S. 38-39.

${ }^{106}$ Shawn William Miller, An environmental history of Latin America, New York, 2007, S. 203.

${ }^{107}$ Paul Leidinger, Paul Leidinger 1994, S. 16.

${ }^{108}$ U. Troitzsch: Historische Umweltforschung: Einleitende Bemerkungen über Forschungsstand und Forschungsaufgaben. In: Technikgeschichte 48(1981), S. 177-190, zitiert nach: Leidinger, S. 19-20.

109 Jürg Helbling, Der Einfluss religiöser Vorstellungen, Normen und Rituale auf die Ressourcennutzung in einfachen Gesellschaften am Beispiel der Cree und der Maring, in: Rolf Peter Sieferle/Helga Breuninger (Hrsg.), Natur-Bilder. Wahrnehmungen von Natur und Umwelt in der Geschichte, Frankfurt/Main 1999, S. 19-42, hier S. 19.
} 
Modell der Welt, innerhalb dessen alle Dinge - inklusive das Tun und Lassen der Menschen, die Natur, die Götter und Geister - ihren Platz haben. Kurz: die religiösen Modelle dienen dazu, dem Leben der Menschen und ihrer Welt Sinn zu geben. Rituale, Normen und Tabus, die jeweils mit einer bestimmten Weltanschauung zusammenhängen, dramatisieren deren Inhalte und machen sie symbolisch kommunizierbar und manipulierbar; sie bestimmen das symbolische Verhalten der Menschen in ihrer sozialen und natürlichen Umwelt. Unmittelbar handlungsrelevant sind hier die Normen, die sich als sanktionierte Handlungsanweisungen definieren lassen.“" ${ }^{110}$ Gerade wenn Umweltgeschichte auch als Lösungslieferant für aktuelle Probleme gesehen wird, erfordert dies die Einbeziehung aller handlungsrelevanten Einstellungen.

\subsubsection{Verbindung natur- und geisteswissenschaftlicher Methoden}

Die eben dargestellte Themenauswahl zeigt das breite Spektrum, das die Umweltgeschichte bietet. Eines jedoch haben alle Fragestellungen gemein: da es stets um Wechselwirkungen zwischen Mensch und Natur geht, werden zur Bearbeitung Methoden sowohl aus dem Bereich der Naturwissenschaften als auch aus den Geisteswissenschaften benötigt. „I.G. Simmons envisions environmental history as a method combining scientific and humanistic approaches and mediating the two." ${ }^{\text {"111 }}$ Auch Herrmann sieht die Kombination von Methoden beider Wissenschaftsbereiche als notwendig für die Umweltgeschichte an: „Die gelegentlich benutzte Unterscheidung in ideographische und nomothetische ${ }^{112}$ Wissenschaftsauffassung läuft bezüglich des Themas ins Leere, weil Umweltgeschichte nicht nur materielle Phänomene, nicht nur erfahrungswissenschaftliche Fragen, sondern auch Normen und Handlungsanweisungen thematisiert einschließlich deren Folgen. (...) Meiner Einschätzung nach sind die Naturwissenschaften eher für die direkten Quellen (mehrheitlich mit Sachüberrestcharakter) zuständig. Die Kulturwissenschaften arbeiten hingegen mit solchen Quellen, die indirekten Charakter haben und mit hermeneutischer Methodik interpretiert werden müssen.“" ${ }^{113}$ Die hier anklingende Trennung bzw. Aufteilung der Forschungsarbeit in natur- und kulturwissenschaftliche Quellenanalyse ist zwar grundsätzlich nachvollziehbar, aber, und hier ist Hermann zu folgen, eine klare Abgrenzung ist bei umweltgeschichtlichen Fragestellungen weder hilfreich noch immer möglich. So dürften die mit naturwissen-

\footnotetext{
${ }^{110}$ Ebenda, S. 21.

${ }^{111}$ Hughes, Hughes 2006, S. 115.

112 Ideographisch: Phänomene in ihrer Besonderheit und Einmaligkeit verstehen. Nomothetisch: zielt auf die Auffindung von Gesetzmäßigkeiten. Dieses Begriffspaar wurde ursprünglich 1894 von Windelband eingeführt, um zwei Wissenschaftstypen zu unterscheiden: einerseits die eher induktive, qualitative Geschichtswissenschaft und andererseits die eher deduktive, quantitative Naturwissenschaft. In Deutschland folgte daraus die Auftrennung der Wissenschaft in Geisteswissenschaften (verstehen) und Naturwissenschaften (erklären).

113 Bernd Herrman, Umweltgeschichte als Integration von Natur- und Kulturwissenschaften, in: Günter Bayerl (Hrsg.), Umweltgeschichte - Methoden, Themen, Potentiale, Münster (u. a.) 1996, S. 21-30, hier S. 21.
} 
schaftlichen Methoden zu Tage geförderten Fakten in den seltensten Fällen selbsterklärend sein. Vielmehr müssen sie erst mit kulturwissenschaftlichen Methoden „zum Sprechen“ gebracht werden. Sieferle argumentiert die Notwendigkeit der Kombination von natur- und geisteswissenschaftlichen Methoden, da zwar eine Rekonstruktion von Naturwahrnehmung auch innerhalb der disziplinären Grenzen der Geschichtswissenschaft möglich ist, aber der Schritt zum Verständnis der Wechselwirkung zwischen Mensch und Natur nicht mehr vollzogen werden kann. „Wie diese mentalen Vorgänge (via Handlungs- oder Verhaltensorientierung) auf reale Naturprozesse gewirkt haben mögen, ist auf dieser Ebene aber häufig nicht mehr zu erklären, da dazu ein Verständnis dieser Prozesse selbst erforderlich wäre." ${ }^{114}$ Erst die Verbindung beider Methodenbaukästen ermöglicht die Erforschung der Wechselwirkungen zwischen Mensch und Natur. ${ }^{115}$

Um Handlungen zu verstehen ist die Interpretation im zeitgeschichtlichen Kontext erforderlich. Ein Naturereignis kann zunächst mit naturwissenschaftlichen Methoden dargestellt werden. ${ }^{116}$ Die Analyse der menschlichen Reaktionen auf dieses Phänomen, respektive Handlungen, erfordert zusätzlich hermeneutische Methoden, denn ohne zeitgeschichtlichen Kontext und Kenntnis der intendierten Wirkung der Handlung der beteiligten Personen (Akteure wie Autor) sind Handlungen nicht zu verstehen. Die Geschichtswissenschaft (als Geisteswissenschaft) könnte sich der naturwissenschaftlichen Herangehensweise nähern, indem sie Fragen nach dem Warum durch Fragen ersetzt, die klären warum Menschen vergangene Ereignisse auf eine bestimmte Weise bewerten. ${ }^{117}$

Die Verbindung von Natur- und Geisteswissenschaftlichen bzw. die Positionierung der Umweltgeschichte in deren Grenzbereich ist jedoch alles andere als unproblematisch. „Offenbar wird die historische Umweltforschung jedoch auch durch Strukturen des Wissenschaftsbetriebs behindert. Umweltgeschichte erfordert Grenzüberschreitung, insbesondere Überschreitung der Grenzen zu den Naturwissenschaften. “" ${ }^{118}$ Radkau weist hier zu Recht darauf hin, dass der heutige Wissenschaftsbetrieb noch immer eine fachliche Spezialisierung der Forschenden präferiert. So dürften ausschließlich disziplinübergreifend arbeitende Wissenschaftler in der Minderheit sein. Auch von Borries sieht für die Umwelt-

\footnotetext{
114 Sieferle, Sieferle 1999, S. 10-11.

${ }^{115}$ Wenngleich der Mensch Teil der Natur ist, drückt diese Begriffskombination sehr gut aus, worum es in der Umweltgeschichte geht. Man könnte auch "Gesellschaft und Umwelt" sagen, aber diese semantischphilosophische Diskussion führte an dieser Stelle zu weit.

${ }^{116}$ Wobei sich auch hier schon die besonderen Fragestellungen der Umweltgeschichte bemerkbar machen. Naturereignisse scheren sich nicht um Ländergrenzen. Dementsprechend machen übliche Kategorien wenig Sinn. „One issue that environmental historians have not systematically confronted is that of scale. (...) But for many sorts of history, including most environmental history, the nation-state is the wrong scale on which to operate. Ecological processes unfold with no regard for borders (...)." McNeill, McNeill 2003, S. 35.

117 Vgl. Topolski, S. 198.

${ }^{118}$ Radkau, Radkau 2000, S. 44.
} 
geschichte die Notwendigkeit, verschiedenste Methoden anzuwenden, um den Fragestellungen gerecht zu werden. „Methodisch überschreitet Umweltgeschichte (...) die klassischen (geisteswissenschaftlichen) Verfahren der Historie. Naturwissenschaftliche Auswertungsmethoden (Pollenanalyse, Dendrochronologie, Skelettuntersuchungen), archäologische Befunde (Siedlungs- und Begräbnisfunde, Ortskerngrabungen, Wüstenforschung, Industriedenkmäler), ikonograhische und kartographische Quellen spielen eine ungewohnt große Rolle. Der multimethodische und interdisziplinäre Zugang droht Lehrende und Lernende zu überfordern.“119 Der daraus abzuleitende Anspruch, diese Vielzahl von Methoden korrekt anwenden oder zumindest auswerten zu können, zeigt den extrem hohen Anspruch umweltgeschichtlicher Fragestellungen an die Forschenden. „Erst eine interdisziplinäre Erforschung der komplexen Verschränkung der Geschichten von Natur, Mensch und Kultur ließe (...) die Konturen einer Umweltgeschichte erkennen. Das sind freilich zumal für Einzelforscher - höchste, vielleicht sogar utopische Ansprüche.“120

In Kapitel 2.1 ist dargestellt worden, dass es nicht die eine Umweltgeschichte gibt, sondern eine Vielzahl von Fragestellungen die aus dem Thema hervorgehen. Dementsprechend kommen Wissenschaftler, die sich mit Umweltgeschichte befassen, aus den verschiedensten Disziplinen. „Es gibt den Umwelthistoriker ebenso wenig wie den Umweltwissenschaftler. (...) Wir sollten uns folglich nicht fragen, was denn nun die Methoden sind, sondern vielmehr, welche Disziplinen mit ihrem methodischen Arsenal Beiträge zum Gegenstand Umweltgeschichte zu leisten imstande sind.“121

\footnotetext{
${ }^{119}$ Bodo von Borries, Didaktische Möglichkeiten und Grenzen der Umweltgeschichte, in: Günter Bayerl (Hrsg.), Umweltgeschichte - Methoden, Themen, Potentiale, Münster (u. a.) 1996, S. 309-326, hier S. 320.

${ }^{120}$ Schenk, Schenk 2008, S. 41.

121 Klaus-Peter Meinicke, Umweltgeschichte aus ingenieurwissenschaftlicher Perspektive, in: Günter Bayerl (Hrsg.), Umweltgeschichte - Methoden, Themen, Potentiale, Münster (u. a.) 1996, S. 285-303, hier S. 285.
} 


\subsection{Werte in der Umweltgeschichte}

\subsubsection{Wertedefinition und -diskussion}

Bevor wir nun die Diskussion um die Existenz und die Stellung von Werten in umweltgeschichtlichen Texten bzw. die Beeinflussung der Forschung durch Werte vertiefen, stellt sich zunächst die Frage: Was eigentlich sind Werte? Mit der Beantwortung dieser Frage lassen sich ganze Bücher füllen, daher soll an dieser Stelle eine kurze Definition zur Annäherung an den Begriff genügen.

Ganz allgemein beschreiben Werte einen wünschenswerten Zustand und beeinflussen damit die Auswahl von Handlungsoptionen. Kluckhohn formuliert Werte als „a conception (...) of the desirable which influences the selection from available modes, means, and ends of action." 122 Schwartz und Bilsky werden mit ihrer Definition spezifischer und grenzen zudem Werte von Einstellungen ${ }^{123}$ ab: „(...) values are (a) concepts or beliefs, (b) about desirable end states or behaviors, (c) that transcend specific situations, (d) guide selection or evaluation of behavior and events, and (e) are ordered by relative importance." 124 Diese Definition scheint alle Aspekte abzudecken, inklusive der hierarchischen Ordnung der Werte. Einige Beispiele, worauf sich Werte beziehen können, listet Mersch auf: „Der Terminus ,Wert' bezeichnet allein auf der soziokulturellen Ebene vielfältige Phänomene - er kann sich auf Interessen, Wohlgefallen, Präferenzen, Pflichten, moralische Bindungen, Verlangen, Wünsche, Ab- oder Zuneigungen beziehen (...).“'125 Eine Abgrenzung erscheint noch erforderlich: die heute gängige Unterscheidung zwischen Wert und Werten, d. h. Wert im ökonomischen Sinn und Werten im moralischen Sinn. „Es erweist sich (...), dass (mindestens) zwei grundsätzlich verschiedene Verwendungsweisen vorherrschen: Nach dem ersten ist ein Wert eine Eigenschaft, die ein Objekt für den Menschen haben kann. Im zweiten Begriffsverständnis sind Werte ${ }^{126}$ Kriterien, die der Mensch hat, um seine Umwelt zu bewerten. “127 In der vorliegenden Arbeit, insbesondere im textanalytischen Teil, werden beide Begriffsverständnisse berücksichtigt. Wie mehrfach geschrieben wurde, beeinflussen

\footnotetext{
${ }^{122}$ Clyde Kluckhohn, Values and Value-Orientations in the Theory of Action: an Exploration in Definition and Classification, in: Talcott Parsons/Edward Shils/Edward C. Tolman (Hrsg.), Toward a general theory of action, New York, NY 1962, 1. ed, hier S. 395.

${ }^{123}$ Einstellungen sind situationsspezifisch, Werte gehen über einzelne Situationen hinaus.

124 Schwartz \& Bilsky 1987, zitiert nach Bertrand Lisbach, Werte und Umweltwahrnehmung: Der Einfluß von Werten auf die Wahrnehmung der natürlichen Umwelt, Marburg, 1999, S. 28.

${ }^{125}$ Mersch, Mersch 2007, S. 11.

126 Zur Bedeutungsentwicklung des Begriffs „Wert“ bzw. „Werte“ siehe den Aufsatz von Schmitt, der die Entwicklung der letzten ca. 1000 Jahre in drei Etappen einteilt. Er stellt fest, dass die Pluralform „Werte“ im Mittelalter noch nicht existierte, wohingegen heute mehr oder weniger strikt zwischen „Wert“ im ökonomischen Sinn und „Werten“ im moralischen Sinn unterschieden wird. Vgl. Jean-Claude Schmitt, Welche Geschichte der Werte?, in: Barbara Stollberg-Rilinger/Thomas Weller (Hrsg.), Wertekonflikte - Deutungskonflikte. Internationales Kolloquium des Sonderforschungsbereichs 496 an der Westfälischen Wilhelms-Universität Münster 19. - 20. Mai 2005, Münster 2007, 1. Aufl., S. 21-35, hier S. 24-26.
}

${ }^{127}$ Lisbach, Lisbach 1999, S. 20. 
Werte das Handeln. Die Basis dieser Beeinflussung besteht laut Rickert in der Geltung der Werte: „Werte sind keine Wirklichkeiten, weder physische noch psychische. Ihr Wesen besteht in ihrer Geltung, nicht in ihrer realen Tatsächlichkeit.“128 Oder wie Rödder es formuliert, müssen Werte „(...) als allgemeine und grundlegende Orientierungsrichtlinien, die für das Denken, Reden und Handeln der Menschen auf individueller und kollektiver Ebene als verbindlich akzeptiert, dabei explizit artikuliert oder implizit angenommen werden." ${ }^{129}$ Die Konsequenz eines akzeptierten Wertes beschreibt Stern folgendermaßen: „Sobald ein Postulat als Norm angenommen und anerkannt ist, erlangen alle mit dieser übereinstimmenden Handlungen, Gegenstände und Aussagen positiven Wert und alle ihr widersprechenden, negativen Wert. (...) Selbstverständlich sind die postulierten Normen selbst Werte." 130 Werte sind also die Basis unseres alltäglichen Handelns und sind daher von grundsätzlich hoher Relevanz bei sämtlichen Analysen menschlichen Verhaltens.

Gleichwohl der Unmöglichkeit einer objektiven Wissenschaft ist die Geschichtswissenschaft anscheinend besonders ausdauernd darin, nach Objektivität zu streben. Sämtliche Methoden zur Quellenkritik sollen den Historiker hierbei unterstützen. Die Umweltgeschichte nimmt hierbei insofern eine besondere Stellung ein, als sie bei vielen Fragestellungen naturwissenschaftliche Quellen bzw. Methoden heranzieht. Aber selbst wenn es dem Forscher gelänge, die inm zur Verfügung stehenden Methoden wertungsfrei anzuwenden, wird inm die vollkommene Objektivität verwehrt bleiben. ${ }^{131}$ Jeder Forscher hat als Individuum verschiedene Stationen der Sozialisation durchlaufen und nimmt durch seinen individuellen Standort in der Gesellschaft auch seine Umwelt auf seine ganz persönliche Weise wahr. Gerade diese Voreingenommenheit befähigt inn aber erst zur Interpretation. Wäre es möglich, einen neutralen Platz außerhalb der Gesellschaft einzunehmen, würde es dem Forscher nichts nutzen, da ihm dann der Referenzrahmen zur Interpretation fehlte. Seine Prägung und das gesellschaftliche Umfeld beeinflussen aber nicht nur, wie etwas wahrgenommen wird, sondern fungieren gleichzeitig als Filter und schränken ein, was wahrgenommen wird. Somit wird auch die Auswahl von Forschungsvorhaben durch Werthaltungen beeinflusst. Das Bemühen um Objektivität wird durch bewusste und unbewusste Faktoren erschwert. Die Faktoren, derer sich der Forscher bewusst ist, lassen sich abschwächen oder auch ganz abschalten. Schwieriger sind die unbewussten Faktoren, das ideologische System, in das man eingebunden ist. Hiermit sind insbesondere derart

\footnotetext{
${ }^{128}$ Rickert et al., S. 111.

${ }^{129}$ Andreas Rödder, Werte und Wertewandel: Historisch-politische Perspektiven, in: Bernhard et al Bueb (Hrsg.), Alte Werte - Neue Werte. Schlaglichter des Wertewandels, Göttingen 2008, S. 9-25, hier S. 12.

130 Stern, S. 251.

131 „Völlig tendenzlos, ,objektiv’ zu berichten, ist keinem Menschen gegeben, auch dann nicht, wenn er sich - wie in der modernen Historiographie - wenigstens ernsthaft darum bemüht." Ahasver von Brandt, Werkzeug des Historikers: Eine Einführung in die historischen Hilfswissenschaften, Stuttgart, 2003, S. 62.
} 
stark verinnerlichte Haltungen gemeint, die so selbstverständlich erscheinen, dass sie nicht mehr hinterfragt werden.

Tatsächlich gehören auch die Wertvorstellungen selbst, die in einer Gesellschaft hinsichtlich ihrer Umwelt existierten, zum Forschungsgebiet der Umweltgeschichte. Problematisch ist das Thema Werte in diesem Zusammenhang, wenn die zugrunde liegenden Werte nicht oder nicht ausreichend definiert sind und ihre Herleitung nicht nachvollziehbar dargestellt wird. „Die Historiker gehen allerdings in der Regel eher unbekümmert mit dem Begriff um. Selten legen sie sich Rechenschaft darüber ab, was Werte eigentlich sind, oder besser: was sie darunter verstehen wollen, und - damit zusammenhängend - wie sich Werte und Wertewandel in der Vergangenheit überhaupt methodisch erfassen und rekonstruieren lassen.“132 Stollberg-Rilinger bezieht sich hier auf gesellschaftliche Werte als Forschungsobjekt, nichtsdestoweniger lässt sich ihre Feststellung auch auf die Wertvorstellungen des Forschers selbst anwenden. Rickert führte schon 1926 aus, dass Forscher sich ihre eigenen Werte genau vor Augen führen sollten. Und nicht nur das; vielmehr sieht er die Begründung, weshalb diese Werte gültig sind als Bestandteil der wissenschaftlichen Praxis an. „Wer Kulturwissenschaft treiben will im höchsten Sinne des Wortes, (...) wird auf die Notwendigkeit geführt, sich auf seine leitenden Kulturwerte zu besinnen und ihre Geltung zu begründen. Das Arbeiten mit unbegründeten Wertsetzungen würde in der Tat der Wissenschaft widersprechen.“133 Auch Marrou fordert die Offenlegung und Erklärung dieser Werte: „Die wissenschaftliche Ehrlichkeit scheint mir zu erfordern, daß der Historiker (...) die Ausrichtung seines Denkens definiert, seine Postulate darlegt (...), daß er sich in Aktion zeigt und uns an der Entwicklung seines Werkes teilnehmen läßt: Warum und wie er seinen Gegenstand ausgewählt und begrenzt hat (...).“134

An dieser Stelle entfernen wir uns kurz von den Werten des Forschers, bzw. denen, die in umweltgeschichtlichen Texten dominieren, hin zu der Frage, warum die Erfassung bzw. die Erforschung und Rekonstruktion der Wertvorstellungen einer Gesellschaft für die umweltgeschichtliche Bearbeitung wichtig ist und daher eine zentrale Rolle in der Umweltgeschichte spielen sollte. Für die Untersuchung der Wechselwirkungen zwischen Gesellschaft und Natur sind die alltäglichen Gewohnheiten der Menschen zentrale Aspekte. Diese Gewohnheiten und Verhaltensmuster werden durch die generalisierten Werte einer Gesellschaft bestimmt. Radkau betont diesen Aspekt denn auch wiederholt: „Gewichtige

\footnotetext{
132 Barbara Stollberg-Rilinger, Die Historiker und die Werte, in: Marie Luisa Allemeyer/Katharina Behrens /Katharina Ulrike Mersch (Hrsg.), Eule oder Nachtigall? Tendenzen und Perspektiven kulturwissenschaftlicher Werteforschung, Göttingen 2007, S. 35-48, hier S. 35.

${ }^{133}$ Rickert et al., S. 170.

134 Henri-Irénée Marrou/Charlotte Beumann, Über die historische Erkenntnis: Welches ist der richtige Gebrauch der Vernunft, wenn sie sich historisch betätigt?, Darmstadt, 1973, Sonderausg. für d. Mitgl. d. Wiss. Buchges. Darmstadt., S. 282.
} 
Gründe sprechen dafür, dass eine abgehobene Ideengeschichte auf die Dauer eine Sackgasse der historischen Umweltforschung darstellt. Entscheidend für den Umgang mit der Umwelt sind die alltäglichen Gewohnheiten, nicht die Ideen großer Geister.“135 „(...) denn es sind nicht einzelne Ideen, sondern dauerhafte in Recht und Gesellschaft verankerte Verhaltensmuster, die die Beziehung zwischen Mensch und Umwelt flächendeckend bestimmen.“136 „Umweltprägend sind in der menschlichen Geschichte nicht die einzelnen Ideen und Aktionen, sondern das Dauerhafte und Flächendeckende: das massenhaft alltägliche, habitualisierte und institutionalisierte Verhalten über lange Zeit.، " ${ }^{137}$ Hier sei darauf hingewiesen, dass individuelle und kollektive Werte nicht deckungsgleich sein müssen. Die Handlungen des Einzelnen müssen daher nicht den Werthaltungen der Gesellschaft entsprechen. Radkau betont die Berücksichtigung der individuellen Sichtweise bei der Quellenkritik: „Um die Quellen richtig zu verstehen, ist es wichtig, daran zu denken, daß der Wald in den Augen des Försters anders aussieht als in denen des Bauern, des Hirten und des erholungssuchenden Städters. “138 Wertvorstellungen sind also ein wichtiger Baustein bei der Rekonstruktion von Verhaltensmustern innerhalb einer Gesellschaft, wenngleich für die Umweltgeschichte die individuellen Werte von Vorrang sind. Auch Hughes hebt die Bedeutung der Wertvorstellungen für die Umweltgeschichte hervor: „A third theme of environmental history is the study of human thoughts about the natural environment and attitudes towards it. (...) It is impossible to understand what has happened to the Earth and its living systems without paying attention to this aspect of social and intellectual history, (...)“139 Die Erforschung der Wertvorstellungen vergangener Gesellschaften oder früherer Zeiten dient aber nicht nur der Rekonstruktion der damaligen Situation und dem Verständnis damaliger Handlungen. Die Kenntnis über gesellschaftliche Wertvorstellungen trägt auch maßgeblich zum Verständnis über die Entwicklung - und ggf. Niedergang - dieser Gesellschaft bei. „Werte sind nicht der Zierrat der Geschichte - sie befehlen, organisieren, hierarchisieren die Vorstellungen der Gesellschaft, sie tragen zur Orientierung bei und zum Urteil über die Entwicklung von Gesellschaften. Daher spielen sie auch eine tragende Rolle für deren Weiterentwicklung.“140 Dem ist zuzustimmen. Und zwar nicht nur was die Erforschung von Werten vergangener und heutiger Gesellschaften betrifft, sondern auch bezüglich der Werte in heutigen wissenschaftlichen Texten, womit wir wieder beim eigentlichen Thema sind.

\footnotetext{
135 Joachim Radkau, Unausdiskutiertes in der Umweltgeschichte, in: Manfred Hettling (Hrsg.), Was ist Gesellschaftsgeschichte? Positionen Themen Analysen ; Hans-Ulrich Wehler zum 60. Geburtstag, München 1991, S. 44-57, hier S. 48.

${ }^{136}$ Radkau, Radkau 2000, S. 47.

${ }^{137}$ Radkau, Radkau 2003, S. 185.

${ }^{138}$ Radkau, Radkau 2000, S. 40.

139 Hughes, Hughes 2006, S. 7.

${ }^{140}$ Schmitt, Schmitt 2007, S. 35.
} 
Bei einer Beschreibung der naturalen Umwelt stellt sich die Frage, inwiefern eine objektive Schilderung möglich ist. „Weil die natürliche Sprache konstative und evaluative Urteile permanent miteinander verschränkt, ist die Beschreibung physikalischer Prozesse in der natürlichen Sprache oft nicht wertneutral.“141 Opp führt aus, dass, wenngleich Wörter sowohl eine konnotative als auch eine denotative Bedeutung haben, sich die konnotative Bedeutung ausschalten ließe, indem der Forscher dies festlegt. Somit wäre eine Trennung von Wertund Sachaussagen möglich und das Wertfreiheitspostulat gültig. ${ }^{142}$ Diesem Ansatz ist nicht zuzustimmen. Nur durch die Festlegung des Forschers, dass ein bestimmtes Wort keine konnotative Bedeutung habe, ist dies im besten Fall für den Forscher selbst gültig, nicht aber für die Leser. Zudem müsste sich der Forscher auch aller impliziten konnotativen Bedeutungen bewusst sein, was wohl kaum möglich ist. „Strauss wies darauf hin, daß die Sprache des Historikers auch dort, wo sie Wertungen vermeidet, voller Werturteile stecke."143 Auch Potthast stellt fest: „Naturwissenschaftliche Begriffe, Methoden und Theorien spiegeln Weltbilder wider, die zuweilen hinter der wissenschaftlichen Fassade unerkannt bleiben." ${ }^{144}$ Insbesondere bei der Beschreibung von Veränderungen in der Natur durch menschliche Einwirkung dürfte sich eine Wertung kaum vermeiden lassen. „Einzig die zur Leitwissenschaft erkorene Physik komme ohne Wertungen aus, während andere Naturwissenschaften - wie etwa die Biologie - schwächeren Objektivitätsidealen folgten und die Natur stärker von ,Subjektvollzügen' her deuteten. Demgemäß fänden sich in den entsprechenden Diskursen immer wieder implizite Wertungen. (...) Der Autor [Mutschler] kommt daher zu dem Schluß, dass eine Beschreibung von Lebensphänomenen ohne eine evaluative Deutung nicht auskommt.“145 Dieses Problem der Objektivität war und ist Kern vieler methodologischer Diskussionen. Die Schlussfolgerung ist nach Marrou die Erkenntnis, dass Objektivität, so erstrebenswert sie auch grundsätzlich sein möge, für Geschichtswissenschaften nicht zielführend ist. „Es war ein Irrweg, die Geschichte auf die Naturwissenschaften auszurichten, die Objektivität zum höchsten und im gewissen Sinne einzigen Kriterium der Wahrheit zu machen." 146

Der folgende Abschnitt soll die Problematik der Bestimmung von Werten, die einem umweltgeschichtlichen Text zugrunde liegen, beleuchten. Dafür wird die grundlegende Problemstellung bei der Ermittlung von Werten aufgezeigt.

\footnotetext{
${ }^{141}$ Mutschler, Mutschler 2007, S. 233.

${ }^{142}$ Vgl. Opp, Opp 2002, S. 227-228.

${ }^{143}$ Karl-Georg Faber, Theorie der Geschichtswissenschaft, München, 1982, 5., erw, S. 180.

144 Thomas Potthast, Wo sich Biologie, Ethik und Naturphilosophie treffen (müssen): Epistemologische und moralphilosophische Aspekte der Umweltethik, in: Konrad Ott/Martin Gorke (Hrsg.), Spektrum der Umweltethik, Marburg 2000, S. 101-141, hier S. 108.

${ }^{145}$ Mersch, Mersch 2007, S. 31.

${ }^{146}$ Marrou et al., S. 261.
} 
Wertvorstellungen einer Gesellschaft zu erfassen ist schwierig, dies gilt insbesondere für vergangene Zeiten. Für historische Forschungen müssen die Werte früherer Gesellschaften unter Zuhilfenahme der verschiedensten Quellen rekonstruiert werden. „For environmental historians, these sources will include not only all the relevant books and articles, but as the case dictates, business records, scientific reports, newspaper reports, and literature revealing the attitudes of people of the times." ${ }^{147}$ Bei der Rekonstruktion müssen auch Hintergründe der Quellen berücksichtigt werden, da die Verfasser oder Auftraggeber zumeist bestimmte Interessen verfolgten. „Durch die schriftlichen Quellen sieht sich der Historiker, sofern er eine Ahnung von Quellenkritik hat, stets auf den Blickwinkel jener Interessen beschränkt, die die Quellen produzierten und tradierten.“" ${ }^{148}$ Zudem sind Forscher durch die eigenen und die aktuellen gesellschaftlichen Wertvorstellungen vorbelastet, was die Rekonstruktion früherer Werte erschwert. „Die Wahl der Brille, durch die wir die Wirklichkeit betrachten, determiniert, was wir sehen, [...] Wählen wir eine andere Brille, so sehen wir die Welt zwar möglicherweise anders, aber ob wir sie besser oder schlechter sehen, können wir nicht entscheiden (da wir die Welt nie ohne Brille sehen).“ ${ }^{149}$ Ist der Forscher sich aber zumindest bewusst, dass er die Wirklichkeit nie gänzlich ungefiltert betrachten kann, eröffnet dies die Möglichkeit, eben diese Filterkriterien bei der Interpretation der betrachteten Wirklichkeit zu berücksichtigen. Des Weiteren könnten Wertvorstellungen indirekt bzw. unbemerkt einfließen. „Wenn Historiker auf eine idealistische Vorstellung von heutigem Umweltbewusstsein fixiert sind, haben sie keinen Blick für die alltäglichen umwelterhaltenden Verhaltensmuster der Vergangenheit, die man oft nur zwischen den Zeilen der schriftlichen Quellen erkennt (...).“150 Paravicini weist in diesem Zusammenhang auf die besondere Problematik der stark verinnerlichten Werthaltungen hin: „Besonders hinderlich sind ungeprüfte Prämissen, gefolgt von (...) den falschen Selbstverständlichkeiten, die stets der Epoche des Betrachters angehören. Sie sind eine ständige Gefahr, selbst für die Tatsachenermittlung, und viel mehr noch für die Tatsachenverwertung.“151

Ein weiteres Problem besteht darin, dass Werte und Handlungen nicht immer deckungsgleich sind; so kann z. B. Nachhaltigkeit als Wert zwar von einer Person im Interview bestätigt werden, aber es werden im Alltag trotzdem Einwegartikel verwendet. Weiterhin ist der Rückschluss von einer Handlung auf einen zugrundeliegenden Wert nicht immer eindeutig: Dämmt z. B. ein Hausbesitzer sein Eigenheim, so kann dies durch Umweltschutzgründe motiviert sein, aber ebenso aus Gründen der Kostenreduzierung. Darüber,

\footnotetext{
${ }^{147}$ Hughes, Hughes 2006, S. 119.

${ }^{148}$ Radkau, Radkau 2003, S. 168.

149 Armin Günther, Technologische Risiken zwischen Wahrnehmung, Konstruktion und Reflexion, in: Martin Stengel (Hrsg.), Umweltökonomie. Eine interdisziplinäre Einführung, München 1997, S. 111-135, hier S. 126.

${ }^{150}$ Radkau, Radkau 2003, S. 167.

${ }^{151}$ Werner Paravicini, Die Wahrheit der Historiker, München, 2010, S. 20.
} 
inwiefern Werte das Verhalten tatsächlich beeinflussen, wird noch diskutiert. Vergleiche hierzu die Modelle von Rohan und von Rokeach zum Zusammenhang von Werten und Handlungen. ${ }^{152}$ So schreibt Helbing über den rein legitimatorischen Bezug auf Werte: „Viele grundsätzliche Überlegungen wurden über den Einfluss von Werten und Normen auf das Verhalten von Akteuren angestellt (...). Gemäß Holy/Stuchlik $(1983,85)$ handeln Akteure aufgrund spezifischer Interessen und Präferenzen, und der Bezug auf Werte und Normen erfolgt lediglich rhetorisch-legitimatorisch im Hinblick auf das Erreichen gesetzter Ziele." ${ }^{153}$

Bei umweltgeschichtlichen Themen kommt eine weitere Schwierigkeit hinzu. Die Wechselwirkungen zwischen Gesellschaft und Natur erfordern oftmals eine Analyse über einen größeren Zeitraum hinweg. Bei längeren Zeiträumen ist somit auch der ggf. stattfindende Wertewandel ${ }^{154}$ einzubeziehen. „Die vorgestellten Ergebnisse und Projekte der historischen Werteforschung zeigen, dass in der Wandelbarkeit des Wertes nicht nur seine kulturhistorische Relevanz verortet, sondern hierin auch seine vermeintliche analytische Unzugänglichkeit begründet ist.“155 Rödder stellt fest: „Denn Werte waren und sind wandelbar. Sie wandeln sich, auf kollektiv-gesellschaftlicher und auf individueller Ebene, sowohl von innen heraus als auch durch äußere Setzung.“" ${ }^{156}$ Eine interessante Wechselwirkung beschreibt Herz, als er als einen Grund für die Änderungen der Wertvorstellungen in den 1950er- bis 1970er-Jahren die veränderten Umweltbedingungen anführt, wobei er insbesondere auf die „düsteren“ Prognosen des Club of Rome verweist. ${ }^{157}$

Die Ermittlung von Werten ist also in mehrfacher Hinsicht problematisch. In der Tat scheint es so zu sein, dass die einer umweltgeschichtlichen Forschungsarbeit zugrunde liegenden Werte selten explizit genannt werden oder die Herleitung nicht nachvollziehbar ist. Auch der eingangs genannte Punkt der definitorischen Schwierigkeit beim Begriff der Werte dürfte dazu beitragen, dass Werte zumeist implizit in einen Text einfließen.

Schließlich muss sich der Umwelthistoriker noch einer letzten Frage stellen, die Smieszek wie folgt beschreibt: „Umweltwissenschaftler(innen), die sich ihrer gesellschaftlichen Rolle bewußt sind und Verantwortung übernehmen möchten, geraten so leicht in einen Konflikt: Der wertneutral kommunizierende Experte steht als aufklärerisches Ideal dem realpolitisch

\footnotetext{
${ }^{152}$ M. Strack/C. Gennerich/N. Hopf, Warum Werte?, in: Erich H. Witte (Hrsg.), Sozialpsychologie und Werte, s.I. 2008, 1. Aufl, S. 91-131, hier S. 93-94.

${ }^{153}$ Helbling, Helbing 1999, S. 22.

154 Dies bezieht sich auch auf die Definition von Wert bzw. Werten. So stellt Stollberg-Rilinger fest, dass die Trennung vom „'Wert der Dinge' im Singular und 'absolute, ethische Werte' im Plural“ (Stollberg-Rilinger, Stollberg-Rilinger 2007, S. 38), also die Trennung des ökonomischen und des ethischen Wertbegriffs, im 19. Jahrhundert vollzogen wurde.

${ }^{155}$ Mersch, Mersch 2007, S. 22.

${ }^{156}$ Rödder, Rödder 2008, S. 13.

157 Vgl. Thomas Herz, Der Wandel der Wertvorstellungen in westlichen Industriegesellschaften, in: Kölner Zeitschrift für Soziologie und Sozialpsychologie (1979) 31, S. 282-302, hier S. 282.
} 
bewußten Wissenschaftler entgegen, der die mediengerechte Darstellung nutzt, um überhaupt erst ein Problembewußtsein zu erzeugen.“158 Was Smieszek eine „mediengerechte Darstellung“ nennt, dürfte nichts anderes als eine Bewertung der wissenschaftlichen Erkenntnisse sein. Potthast benennt gleich drei Fehlschlüsse, die der Forscher versucht sein könnte zu ziehen: Zunächst der Sein-Sollen-Fehlschluss nach Hume ${ }^{159}$, des Weiteren der naturalistische Fehlschluss nach Moore: „Auch Natürlichkeit an sich hat keine moralische Relevanz. Die Ableitung von Werten oder Normen aus der Tatsache der ,Natürlichkeit' einer Eigenschaft oder eines Objekts bedeutet einen naturalistischen Feh/schluß [Hervorh.i.O.] nach George Edward Moore." ${ }^{160}$ und schließlich die Ableitung von Werten aus vorhandener Geschichte oder Tradition. ${ }^{161}$ Zum naturalistischen Fehlschluss schreibt Poser, dass u. a. in den Geschichtswissenschaften deskriptive und normative Elemente gemischt vorkommen, dies den Forscher aber nicht von der Aufgabe enthebt, „(...) jeweils zu klären, ob ein Prädikat oder eine Aussage deskriptiver oder präskriptiver Natur ist.“162 Der Forscher sollte also nicht nur während einer Analyse auf einfließende Wertungen achten bzw. diese vermeiden, sondern sich gerade bei den Schlussfolgerungen und der Art der Ergebnisdarstellung bewusst sein, welche Fehlschlüsse (s.o.) zu vermeiden sind. Zumindest sollte eine Bewertung von Ergebnissen aber bewusst und möglichst getrennt erfolgen: Wissenschaft und Politik sollten nicht vermischt werden. So kommt auch Smieszek zu dem Schluss, dass die Bewertung nicht Aufgabe der Wissenschaft ist, sondern Sache der Gesamtgesellschaft. ${ }^{163}$

\subsubsection{Forscherpositionen in verschiedenen Epochen und Disziplinen}

Die Diskussion um Werte bzw. um Wertfreiheit in der Wissenschaft zieht sich durch einige Epochen und berührt viele Disziplinen. Es gibt zu diesem Thema entsprechend zahlreiche Positionen. In diesem Abschnitt sollen einige vorgestellt werden.

Beginnen wir mit Strauss, der feststellt, dass wissenschaftliche Erkenntnis an sich einen Wert darstellt: „Aber die Wichtigkeit oder die Bedeutung jeder Erkenntnis hängt von Wertideen und damit von historisch veränderlichen Prinzipien ab. Letztlich trifft dies auf jede Wissenschaft zu. Alle Wissenschaft setzt voraus, daß Wissenschaft wertvoll ist; doch ist

\footnotetext{
158 Timo Smieszek, Unsicherheit, Werthaltungen und Handlungsblockaden, in: GAIA 15 (2006) 4, S. 251-254, hier S. 252.

159 Danach kann logisch nicht von einer deskriptiven Aussage (Sein) auf eine normative Aussage (Sollen) geschlossen werden.

${ }^{160}$ Potthast, Potthast 2000, S. 106.

${ }^{161}$ Vgl. ebenda, S. 106-107.

162 Hans Poser, Wissenschaftstheorie: Eine philosophische Einführung, Stuttgart, 2001, S. 36.

${ }^{163}$ Vgl. Smieszek, Smieszek 2006, S. 254.
} 
diese Voraussetzung das Produkt gewisser Kulturen und damit historisch relativ. " ${ }^{164}$ Herder befindet, dass jede Epoche mit den ihr eigenen Werten betrachtet werden müsse und kommt zu dem Schluss, dass eine objektive Geschichtsbetrachtung nicht möglich ist. Seine Überlegungen finden sich später im Historismus wieder. ${ }^{165}$ Auch bei Ranke wird dies betont: „Die moralischen Maßstäbe der Gegenwart dürften nicht auf die Vergangenheit angewendet werden; allgemeingültige Grundsätze dafür gäbe es nicht.“" ${ }^{166}$ Rickert konstatiert ähnlich: „(...) so kommt als wahr in der Geschichte nur noch das rein tatsächliche in Betracht. Alle historischen Begriffe dagegen gelten dann nur eine bestimmte Zeit, und das heißt, sie gelten als Wahrheiten überhaupt nicht, denn sie haben zu dem, was absolut oder zeitlos gilt, kein bestimmtes Verhältnis.“ ${ }^{167}$ Troeltsch versuchte sich später an einem moralischen Modell zur Beurteilung einzelner Epochen. Da dies aber absolut gültige Werte erforderte, scheiterte er mit diesem Ansinnen. ${ }^{168}$ Dementgegen existieren für Stern durchaus übergeordnete, objektive Werte: „Indem die Menschen aller Zeiten, Kulturen, Landstriche und sozialen Schichten die fundamentale Daseinsbedingung ihrer Gattung akzeptieren (...), bekunden sie den Willen, ihr gerecht zu werden. Dies allen Menschen gemeinsame Daseinsprojekt zeigt, daß sie dem Leben und der Gesundheit stets einen positiven Wert, dem Leiden und dem Tode aber einen negativen Wert zuschrieben und auch weiterhin zuschreiben. Darum betrachten wir diese an das Lebensprojekt geknüpften existentiellen [Hervorh. i. O.] Werte ${ }^{169}$ als übergeschichtliche [Hervorh. i. O.] Werte, die von den verschiedenen Epochen, Kulturen und sozialen Bedingungen unabhängig sind. Obgleich diese existentiellen Werte nicht zahlreich sind, haben sie dennoch große theoretische Bedeutung; denn sie stellen übergeschichtliche [Hervorh. i. O.], objektive Maßstäbe dar.“170 Insofern der Mensch am Fortbestand seiner Spezies interessiert ist muss sein Wertesystem dieses Ziel widerspiegeln, denn „Ein mit diesen objektiven Erfordernissen unvereinbares Wertsystem würde, auf die Dauer, die Erhaltung des Lebens unmöglich machen.“ ${ }^{171}$ Es können demnach nur solche Werte von Dauer sein, die sich logisch zwingend aus der Existenzerhaltung ergeben. Direkt sind das wie o.g. Leben und Gesundheit. Um aber Leben und Gesundheit zu

\footnotetext{
164 Leo Strauss, Die Unterscheidung zwischen Tatsachen und Werten, in: Hans Albert/Ernst Tropisch (Hrsg.), Werturteilsstreit, Darmstadt 1979, S. 73-91, hier S. 73.

${ }^{165}$ Vgl. Lothar Kolmer, Geschichtstheorien, Paderborn, 2008, S. 45-47.

${ }^{166}$ Ebenda, S. 49.

${ }^{167}$ Rickert et al., S. 164-165.

${ }^{168}$ Kolmer, Kolmer 2008, S. 54.

${ }^{169}$ Dieses Konzept der existenziellen Werte „Leben“ und „Gesundheit“ (des Menschen) wird bei der Textanalyse in Kapitel 4 integriert, da es sich meines Erachtens zwar vielleicht nicht um objektive Maßstäbe handelt, aber zumindest dürfte die übergeschichtliche Gültigkeit dieser Werte unstrittig sein.

170 Stern, S. 244.

${ }^{171}$ Ebenda, S. 247.
} 
erhalten, müssen entsprechend günstige Bedingungen geschaffen werden, was die Einhaltung weiterer Werte wie z. B. Nachhaltigkeit erfordert.

Weber forderte, dass Wissenschaft werturteilsfrei sein muss. Dies bezieht er besonders auf die Entwicklung von bindenden Werten und Normen, denn nach seiner Auffassung steht es dem Forscher nicht zu, über den Sinn eines Ziels zu urteilen, sondern der Wissenschaftler kann nur die Eignung der Mittel zur Erreichung dieses Ziels prüfen. Anders gesagt ist es nicht Aufgabe der Forscher, Aussagen zum „Soll“ zu treffen, sondern ausschließlich zum „Ist“. Nach Weber müssen Werturteile aber nicht ausgeschlossen werden, jedoch fordert er, dass die Wertprämissen klar dargelegt werden. ${ }^{172}$ Er plädiert für die klare Trennung von Werten und objektiven Aussagen. Weber nimmt an, dass objektive Wissenschaft möglich ist und zwar durch die Trennung von Forscher und wollendem Menschen. Die hier unterstellte Wertfreiheit des Menschen als Forscher ist meines Erachtens allerdings kritisch zu sehen, da es kaum möglich sein dürfte, sämtliche persönliche Werthaltungen auszuschalten bzw. darzulegen und diese nicht in die Forschungsarbeit einfließen zu lassen. Zumindest gilt dies für die Geisteswissenschaften. „Putnam vertritt allgemein die These, dass Fakt und Wert nicht getrennt werden können, weil sie [die Menschen, A. d. V.] niemals im Stande sind, wertfrei zu urteilen. Dies hieße, dass die Differenz zwischen konstativen und evaluativen Urteilen eine rein epistemische und keine ontologische Differenz bezeichnet." ${ }^{173}$ So führt auch Feix mit Bezug auf Schmoller aus: „Beobachtung, Beschreibung, Klassifikation und Verursachungssystem sind in den Geisteswissenschaften nur durch teleologische Zusammenhänge und durch Beziehung auf Werte möglich. Die Lehre der Unmöglichkeit der Werturteilsfreiheit ist also eine konsequente Haltung in den erkenntnistheoretischen Ansätzen der jüngeren historischen Schule. Die Notwendigkeit, wertend zu denken, wird klar postuliert.“174

„Das Ziel der wissenschaftlichen Tätigkeit ist umfassende, informative, gehaltvolle Wahrheit und Gültigkeit. Ziele sind nun höchste oder letzte Werte in Bezug auf das, wofür sie Ziele sind. Deshalb ist (...) Wahrheit und Gültigkeit ein höchster Wert für die Wissenschaft. Und das heißt, daß es keinen solchen übergeordneten Wert für die Wissenschaft geben darf, dem Wahrheit und Gültigkeit geopfert werden. Diese Forderung ist eine [Hervorh. i. O.] mögliche Form des Wertfreiheitspostulats der Wissenschaft." ${ }^{175}$ In letzter logischer Konsequenz bedeutet dies allerdings, dass keine Wissenschaft wertfrei sein kann, da ihr Ziel

\footnotetext{
172 "Es ist die Anerkennung zeitloser Werte, die Webers Position am deutlichsten vom Historismus unterscheidet." Strauss, S. 73.

${ }^{173}$ Mutschler, Mutschler 2007, S. 223.

${ }^{174}$ Nereu Feix, Werturteil, Politik und Wirtschaft, Göttingen, 1978, S. 25.

${ }^{175}$ Paul Weingartner, Werte in den Wissenschaften, in: Franz-Martin Schmoelz/Paul Weingartner (Hrsg.), Werte in den Wissenschaften. Festschrift zum 30jährigen Bestehen des Internationalen Forschungszentrums in Salzburg, Innsbruck 1991, S. 11-41, hier S. 13.
} 
bereits einen Wert darstellt. Auch Schmidt weist zu Recht darauf hin, dass Wissenschaft immer Werte enthält: „Wenn eine Methode ein Mittel zum Erreichen eines Zieles ist, enthält sie Vorschriften über die Wege, die man gehen muß, um das Ziel zu erreichen. Diese ,Soll'Vorschriften liefern uns die für die wissenschaftliche Arbeit nötigen ethischen Normen. Das ist die Stelle, wo, und die Art, wie die Wissenschaft Werte enthält." ${ }^{176}$ Demgegenüber sieht Weingartner auch wertfreie Disziplinen: „So ist die Gruppe der teleologischen Wissenschaften - zu denen z. B. die Geschichte gehört - mehr wertgeladen (weniger wertfrei) als die Gruppe derjenigen, die überhaupt keine Wertaussagen in den Begründungen für ihre Aussagen verwenden, wie z. B. die Mathematik oder die Physik.“"177

Faber beschreibt die erforderliche Unterscheidung von wissenschaftsimmanenten Beurteilungen und normativen Urteilen. ${ }^{178}$ Damit meint er, dass eine wissenschaftliche Beurteilung eines Sachverhalts nicht zwangsläufig eine Bewertung sein muss. So kann z. B. die Umschreibung eines Phänomens als „außergewöhnlich“ durchaus als sachliche Beurteilung bzw. als Einordnung des Phänomens angesehen werden.

Gadamer führt aus, „(...) daß die zeitliche Distanz der Historiker von den Texten, die sie untersuchen, ihre Lesarten fundamental anders ausfallen läßt als die eines Zeitgenossen. Historiker können gleichzeitig verstehen, was der zeitgenössische Zeuge nicht verstanden hätte, und müssen gleichwohl den Text aus der Perspektive der Kultur lesen, in der sie selbst beheimatet sind.“179 So folgert Gadamer denn auch: „Ein Absolutes, für alle Zeiten gültiges Verständnis einer Sache sei unmöglich; jede Zeit verstehe einen überlieferten Text auf ihre Weise neu. “180 Ein wichtiger Aspekt in der Auslegung von Texten ist für inn der Hermeneutische Zirkel, denn ohne (Vor)Wissen ist kein Wissen möglich. Ergo ist immer schon etwas Wissen bzw. eine Meinung zu einem Sachverhalt vorhanden, bevor sich der Wissenschaftler der Interpretation einer Quelle widmet. „Diese Vorannahmen muss man sich bewusst machen, um sie zu kontrollieren. Gadamer meinte, dass gerade dadurch Verstehen ermöglicht werde.“" ${ }^{181}$

Damit sind wir bei der Abgrenzung von Erklären und Verstehen. „In der Wissenschaft spielen Prozesse des Verstehens und Erklärens eine besondere Rolle. Sie bezeichnen die Haupttätigkeit des Wissenschaftlers. Indem er Wissenschaft betreibt, versucht er Vorgänge

\footnotetext{
${ }^{176}$ Paul F. Schmidt, Ethische Normen in der wissenschaftlichen Methode, in: Hans Albert/Ernst Tropisch (Hrsg.), Werturteilsstreit, Darmstadt 1979, S. 353-364, hier S. 358.

177 Weingartner, S. 40.

${ }^{178}$ Vgl. Faber, S. $170 \mathrm{f}$.

${ }^{179}$ Howell, Howell 2004, S. 129.

${ }^{180}$ Kolmer, Kolmer 2008, S. 59.

${ }^{181}$ Ebenda, S. 60.
} 
zu verstehen und Zusammenhänge zu erklären.“182 Im 19. Jahrhundert wurde der Begriff des "Verstehens" zunehmend den Geisteswissenschaften zugeordnet und der des „Erklärens" den Naturwissenschaften. Die Zuordnung des Verstehens zu den Geisteswissenschaften kann auf Friedrich Schleiermacher zurückgeführt werden. Schleiermacher ging davon aus, dass Menschen sich missverstehen können, da sie sich zunächst immer fremd sind. Daraus leitete er seine Forderung ab, dass der Forscher sich in den Autor des untersuchten Textes hineinversetzen müsse; inn schließlich sogar besser zu verstehen, als er sich selbst. „Für Droysen (...) ermöglichte die Methode des Verstehens noch objektives Wissen, da er fest daran glaubte, in einer von Gott durchdrungenen Welt zu leben. (...) Er erweiterte die historische Methode um hermeneutische Aspekte, thematisierte die Vorgänge des Verstehens und koppelte sie an das Subjekt, die Person des Verstehenden.“183 Auch Weber schrieb zum Thema „Erklären und Verstehen“, wobei er weniger die Trennung suchte, sondern vielmehr die Integration beider Ansätze zum Vorteil der Geschichtswissenschaft. „Webers ,verstehende Soziologie' basierte auf der Prämisse, daß ein durch Deutung gewonnenes 'Verständnis' menschlichen Verhaltens durchaus eine erhebliche qualitative Evidenz haben könne, doch müsse das ,Verstehen' des Zusammenhangs noch mit den sonst üblichen Methoden kausaler Zurechnung (...) kontrolliert werden, ehe eine noch so evidente Deutung zur gültigen, verständlichen' Erklärung werde. Webers ,verstehende Soziologie' ordnete das Verstehen also dem Erklären unter und erblickte die Hilfsfunktion des Verstehens erstens in der Ermittlung des zeitgenössischen Selbstverständnisses (...) und zweitens in der Deutung eines historischen Ereignisses (...) unter übergreifenden Gesichtspunkten der jeweiligen Zeit. Weber sprach daher auch von ,erklärendem Verstehen'." 184

Sieferle führt an, dass die umweltgeschichtlichen Arbeiten in den 1980er-Jahren eine neue Perspektive einnehmen. Dieser neue Ansatz beruht auf meist impliziten normativen Vorstellungen einer heilen Natur, die durch den Menschen beeinträchtigt wird. Insbesondere zeigt sich dieser Ansatz in Abhandlungen zu aktuellen Umweltproblemen. ${ }^{185}$ Potthast stellt in diesem Zusammenhang fest: „Wenn Naturwissenschaft sich als empirische wertfreie Wissenschaft ernst nimmt, gibt es keinerlei moralisch zu bevorzugende Zustände ökologischer Systeme.“" 186

Zusammenfassend lässt sich feststellen, dass die Problematik der Wertfreiheit sehr intensiv und schon über einen langen Zeitraum diskutiert wird. Es scheint weitgehend Einigkeit

\footnotetext{
182 Ludolf Herbst, Komplexität und Chaos: Grundzüge einer Theorie der Geschichte, München, 2004, S. 58.

${ }^{183}$ Kolmer, Kolmer 2008, S. 50-51.

${ }^{184}$ Herbst, Herbst 2004, S. 71-72.

${ }^{185}$ Vgl. Rolf Peter Sieferle, Die Grenzen der Umweltgeschichte, in: GAIA 2 (1993) 1, S. 8-21, hier S. 9.

${ }^{186}$ Potthast, Potthast 2000, S. 106.
} 
darüber zu herrschen, dass Objektivität in der Geschichtswissenschaft weder möglich noch zielführend ist. Wichtig ist jedoch, dass sich die Forscher der Werteproblematik bewusst sind.

Im nächsten Abschnitt geht es um die Frage, warum Werte so schwierig zu identifizieren sind und, daraus folgend, wie Werte, insbesondere die impliziten, sichtbar werden.

\subsubsection{Werteidentifikation, insbesondere von impliziten Werten}

„Doch das Wesen der Dinge - so ein berühmtes Diktum von Heraklit - versteckt sich gern. (...) sie verstecken sich in der Sprache, mit der man sich ausdrückt, weil sich die Wahrheit von etwas immer ,sagen' lassen muss - und oft ist die ,Art', sie zu sagen, selbst eine Wahrheit." ${ }^{187}$ Im Vergleich zum reinen Informationsgehalt einer Aussage ist es also mitunter aufschlussreicher, wie etwas gesagt wird. Dies trifft auf das gesprochene Wort stärker zu als auf das geschriebene, da in Gespräch oder Vortrag Mimik und Gestik die sprachlichen Mittel ergänzen. Aber auch das geschriebene Wort bietet reichlich Spielraum, um verschiedenste Wertungsausprägungen auszudrücken ohne dies direkt zu tun. Gerade diese Zusatzinformationen sind es aber, die benötigt werden, um die eigentlichen Informationen im richtigen Kontext zu interpretieren. ${ }^{188}$ „Weil wir keinen unmittelbaren Zugriff auf die Sinnesund Bewusstseinsleistungen der sozialen Anderen haben, sind wir auf die symbolischen Objektivationen angewiesen, in denen Handlungsabsichten, Erfahrungen, Wissen und Imaginationen zum Ausdruck gebracht, damit sinnlich und intersubjektiv zugänglich gemacht werden.“" ${ }^{189}$ In den Sozialwissenschaften ist diese Problematik ${ }^{190}$ stärker präsent als in den Geschichtswissenschaften; in dem Moment jedoch, wo hermeneutische Ansätze erforderlich sind, steht auch der Geschichtswissenschaftler vor der Frage, wie die Quelle korrekt auszulegen ist. Wie aber kann von einem Text auf die zugrunde liegenden Werte geschlossen werden, zumal diese dem Autor selbst oftmals nicht bewusst sein dürften?

\footnotetext{
${ }^{187}$ Castelli Gattinara, S. 215-216. Der zitierte Ausspruch von Heraklit bezieht sich auf DK 22 B 123. Gelegentlich wird über die korrekte Übersetzung ins Deutsche diskutiert, eine andere Transkription lautet beispielsweise „Natur pflegt sich versteckt zu halten“.

188 Dies mag einer der Gründe sein, weshalb der persönliche Kontakt trotz mittlerweile guter technischer Kommunikationsmöglichkeiten noch immer von den meisten vorgezogen wird, zumindest wenn es um wichtige Themen geht.

${ }^{189}$ Soeffner/Raab, Kulturwissenschaftliche Methoden und Ansätze in den Disziplinen, in: Friedrich Jaeger/Jürgen Straub (Hrsg.), Handbuch der Kulturwissenschaften. Band 3. Paradigmen und Disziplinen 2004, S. 552-567, hier S. 552.

190 Die Daten des Sozialwissenschaftlers „sind demnach - anders als die des Naturwissenschaftlers vorinterpretiert, weshalb es sich bei innen in sozialwissenschaftlicher Perspektive um ,Konstruktionen erster Ordnung' handelt. (...) Der Sozialwissenschaftler entwirft kontrollierte, methodisch überprüfte und überprüfbare, verstehende Rekonstruktionen der Konstruktionen erster Ordnung - die ,Konstruktionen zweiter Ordnung'. (...) Die Deutungsarbeit (...) ist die Rekonstruktion von Prozessen der Sinnkonstitution und daher nicht nur Anleitung zum Verstehen von etwas, von einem Inhalt, sondern auch Aufklärung über die Prozeduren und Perspektiven des Verstehens selbst - sie ist ein Verstehen des Verstehens." Ebenda, S. 552-553.
} 
„Menschen tun in aller Regel, so sagen es die empirischen Erkenntnisse der Sozialpsychologie, was allgemein bzw. was in ihrer Umgebung als richtig angesehen wird, sprich: was der so empfundenen bzw. der gesetzten "Normalität“ entspricht. Normalität enthält den Begriff der Norm - und in der Tat stehen die Ebenen der immateriellen Werte und des konkreten Handelns in engem Zusammenhang und Wechselverhältnis. “191 Und Rödder führt weiter aus: „Sozialpsychologische Experimente zeigen aber, wie sehr die umgebende Normalität den Menschen prägt und wie schwer demgegenüber individuelles nonkonformes Handeln fällt - und nur wenige tun dies. (...) Auch heute ist die kollektive, die gesamtgesellschaftlich akzeptierte Normalität und Norm handlungsleitend - nur merken wir es in der Regel gar nicht (...).“192 Gesellschaftliche Normen leiten sich aus den akzeptierten Werten einer Gesellschaft ab. Somit lässt das alltägliche (nicht mehr hinterfragte) Handeln einen Rückschluss auf zugrundeliegende Werte zu. Kruse beschreibt dies folgendermaßen: „Indem der einzelne die gesellschaftlich konstituierten Werte in individuelle ,Richtgrößen' transformiert, schreiben wir ihm eine ,Werthaltung' zu, ein individuelles Wertsystem, das seine Deutungen und Bewertungen von Ereignissen sowie seine Entscheidungen und Handlungen mit den Interpretationen und Handlungen der anderen vergleichbar und bewertbar macht - für inn selbst und für die anderen.“193 Diese Ansätze kann sich der Historiker zunutze machen indem er geeignete Methoden entwickelt die sich an seinen Erkenntnisansprüchen orientieren. Mersch sieht diese Übersetzungsleistung „(...) in ein Instrumentarium der historischen Forschung (...) als elementare Eigenleistung der historisch arbeitenden Disziplinen." ${ }^{194}$ Proctor zeigt diesbezüglich einen sehr praktisch orientierten Ansatz auf: „Another way to think of ethics is that it involves thinking about caring. We all care deeply about some things and not others, and we care or don't care about these things in particular ways and for particular reasons. Ethics involves sorting out why people care as they do (...). ${ }^{195}$ In seinem Aufsatz zur sozial konstruierten Natur arbeitet Proctor an einem Beispiel heraus, dass auch im ersten Moment scheinbar wertfreie wissenschaftliche Abhandlungen bestimmte implizite Werthaltungen beinhalten. Diese werden als Prämissen zwar nicht explizit genannt, können aber durch Interpretation der Art der Ergebnisformulierung identifiziert werden. ${ }^{196}$ Hier wird auch deutlich, dass eine Abhandlung

\footnotetext{
${ }^{191}$ Rödder, Rödder 2008, S. 14.

192 Ebenda, S. 16.

${ }^{193}$ Kruse et al., Kruse et al. 1990, S. 633.

194 Mersch, Mersch 2007, S. 12.

195 James D. Proctor, Solid Rock and Shifting Sands: The Moral Paradox of Saving a Socially Constructed Nature, in: Noel Castree/Bruce Braun (Hrsg.), Social nature. Theory, practice, and politics, Malden, Mass. 2001, S. 225-239, hier S. 227.

${ }^{196}$ Vgl. ebenda, S. 225-239.
} 
durchaus einen Umweltschaden unterstellen kann, ohne dies explizit zu benennen. ${ }^{197}$ Wird z.B. das Ergebnis einer Studie so formuliert, dass beim Leser der Eindruck entsteht, dass Handlungsbedarf erforderlich ist, scheint das analysierte Phänomen als unerwünscht oder als schädlich eingeordnet worden zu sein. Dies wiederum setzt eine bestimmte Werthaltung des Autors der Studie gegenüber dem vom Phänomen betroffenen Umweltelement voraus. Wird in einem Text also ein Phänomen als Umweltschaden thematisiert, auch ohne dies explizit so zu benennen, lassen sich daraus bestimmte Werte ableiten. Daraus folgt, dass es keine zwingende Voraussetzung für die Analyse eines Textes ist, dass das Wort „Umweltschaden“ in diesem Text vorkommt. Dieser Punkt wird in Abschnitt 3.2.1 weiter ausgeführt.

Zusammenfassend kann festgestellt werden, dass Werte selten oder gar nicht direkt aus einem Text ersichtlich sind, dies gilt insbesondere für implizite Werte. Daher wird für die Ermittlung von Werten ein Indikator verwendet, der auf bestimmte Werthaltungen hinweisen kann. Dieser Indikator ist in der vorliegenden Untersuchung der Begriff „Umweltschaden“, da ein Schaden unerwünschte Folgen eines Ereignisses bezeichnet und die Gründe für diese Unerwünschtheit in den zugrunde liegenden Werten zu finden sind. Diese Zusammenfassung des Grundgedankens soll im folgenden Abschnitt vertieft werden.

\subsubsection{Zum Begriff Umweltschaden}

Im Folgenden soll gezeigt werden warum sich gerade der Begriff „Umweltschaden“ besonders eignet, um implizite Werthaltungen in Texten zu ermitteln. Dafür nähern wir uns dem Begriff zunächst über die Diskussion verschiedener Definitionsansätze und Erläuterungen zu den darin enthaltenen Begriffen. Anschließend wird der Logikschluss vom Umweltschaden auf die zugrunde liegenden Werte gezeigt und die gängigen Wertkategorien der Ökologischen Ökonomie werden dargestellt und diskutiert.

Was also ist ein Umweltschaden? Zunächst einmal kann ein Schaden allgemein als die durch eine Ereignisfolge eintretende Veränderung an einem Schutzgut definiert werden, die einen Nutzen mindert. ${ }^{198}$ Daraus folgt mit Bezug auf das Schutzgut „Umwelt“ aus Sicht der Ökologischen Ökonomie: „Ein ökologischer Schaden ist eine unfreiwillige Einbuße der

\footnotetext{
${ }^{197}$ Radkau stellt hierzu fest: „Aber der Umwelthistoriker muß sich ohnehin daran gewöhnen, zwischen den Zeilen zu lesen." Joachim Radkau, Was ist Umweltgeschichte?, in: Werner Abelshauser (Hrsg.), Umweltgeschichte. Umweltverträgliches Wirtschaften in historischer Perspektive ; acht Beiträge, Göttingen 1994, S. 11-28, hier S. 22.

198 Vgl. Broder Breckling/Thomas Potthast, Der ökologische Schadensbegriff - eine Einführung, in: Thomas Potthast (Hrsg.), Ökologische Schäden. Begriffliche, methodologische und ethische Aspekte 2004, S. 1-16, hier S. 7.
} 
Versorgung mit ökologischen Gütern, die durch ein Ereignis hervorgerufen wird." 199 An dieser Stelle sei darauf hingewiesen, dass die Begriffe „Umweltschaden“ und „ökologischer Schaden" in der vorliegenden Arbeit synonym verwendet werden. Der Versuch einer begriffstheoretischen Abgrenzung erscheint wenig zielführend. Zum einen, da zu keinem der beiden Begriffe eine eindeutige, allgemein anerkannte Definition vorliegt. Zum anderen, da der Ansatz zur Abgrenzung, dass „(...) ein Schaden entweder (nur) für die ,Umwelt‘ von Menschen oder (allein) bereits unabhängig von ihrer Nutzung ,ökologisch’ existiert (...) “200 für die Ermittlung der zugrundeliegenden Werte unerheblich ist und zudem der Nachweis, dass ein Schaden völlig unabhängig von der (menschlichen) Nutzung ${ }^{201}$ existiert, schwierig zu führen sein dürfte.

„Umweltschaden“ meint hier Schaden im weiteren Sinne, da ein Umweltschaden sowohl Schäden an der Natur selbst als auch Schäden durch Einschränkung oder Verlust der Nutzung durch Menschen (sowohl aktuelle als auch potenzielle/zukünftige Nutzung) bedeuten kann, wobei Umwelt nicht nur Natur meint, sondern auch von Menschen gestaltete Umwelt wie z. B. Häuser, Straßen, landwirtschaftliche Anlagen oder touristische Angebote. Damit ist auch das oben erwähnte Schutzgut (Gut als Mittel zur Bedürfnisbefriedigung) beschrieben.

Das Umweltschadensgesetz (Gesetz über die Vermeidung und Sanierung von Umweltschäden, USchadG) definiert eine Bodenschädigung nur dann als Umweltschaden, wenn „eine Schädigung des Bodens durch eine Beeinträchtigung der Bodenfunktion (...), die durch direkte oder indirekte Einbringung von Stoffen (...) hervorgerufen wurde und Gefahren für die menschliche Gesundheit verursacht." ${ }^{202}$ Interessant ist hier die Verknüpfung durch „und“, denn dadurch wird der anthropozentrische Ansatz dieses Gesetzes überdeutlich. Auf diesen Punkt kommen wir später zurück.

Das Bundesnaturschutzgesetz (Gesetz über Naturschutz und Landschaftspflege, BNatSchG) definiert in Paragraf 19 nicht nur den Begriff Schaden i. S. d. BNatSchG, sondern beschreibt

\footnotetext{
199 Jan Barkmann/Rainer Marggraf, Ökologische Schäden durch Vernachlässigung des Vorsorgeprinzips im nachhaltigen Landschaftsmanagement - eine umweltökonomische Perspektive, in: Thomas Potthast (Hrsg.), Ökologische Schäden. Begriffliche, methodologische und ethische Aspekte 2004, S. 57-76, hier S. 65.

200 Thomas Potthast, Ökologische Schäden - eine Synopse begrifflicher, methodologischer und ethischer Aspekte, in: ders., Ökologische Schäden. Begriffliche, methodologische und ethische Aspekte, S. 189-209, hier S. 206.

${ }^{201}$ Zumindest wenn auch nicht-nutzungsabhängige Werte herangezogen werden (existence values und bequest values). Aber auch schon bei indirekter Nutzung z.B. als Erosionsschutz ist der Nutzen für den Menschen gegeben. Vgl. dazu auch weiter unten den Abschnitt zu Wertkategorien der Ökonomischen Ökologie. Zudem müsste auch berücksichtigt werden, dass Nutzung und Nutzen zwei unterschiedliche Begriffe sind (vgl. dazu: Krause/Marggraf/Meyer, Kann die Umweltökonomie den intrinsischen Wert berücksichtigen?, in: Zeitschrift für Umweltpolitik und Umweltrecht (2008) 31, S. 293-313, hier S. 296-298), aber sowohl die Nutzung durch den Menschen als auch der Nutzen für inn sind für die Abgrenzung zwischen Umweltschaden und ökologischem Schaden von Belang.

202 Umweltschadensgesetz: §2 Nr. 1 lit. c USchadG, 20.04.2013.
} 
auch, wie zu ermitteln ist, ob ein solcher Schaden vorliegt: „Ob Auswirkungen nach Absatz 1 erheblich sind, ist mit Bezug auf den Ausgangszustand (...) zu ermitteln.“203 Des Weiteren liegt eine Erheblichkeit ${ }^{204}$ u. a. nicht vor bei „nachteiligen Abweichungen, die geringer sind als die natürlichen Fluktuationen, die für den betreffenden Lebensraum oder die betroffene Art als normal gelten oder die auf natürliche Ursachen zurückzuführen sind (...).“205

Diese kurzen Gesetzesauszüge benennen mehrere Schwierigkeiten bei der Bestimmung eines Umweltschadens. Zunächst einmal die Definition des Ausgangszustandes, auch Referenzzustand genannt. Um einen Zustand unter den Begriff Umweltschaden subsumieren zu können, muss also der Vergleich des jetzigen Zustandes mit dem Ausgangsoder Referenzzustand ergeben, dass eine erhebliche Beeinträchtigung der Funktion vorliegt. Der Ausgangszustand liegt zeitlich vor dem Schadensereignis. Die Ermittlung des Referenzzustandes vor Eintritt des Schadensereignisses spielt neben der Erhebung des aktuellen Zustandes daher eine zentrale Rolle in der Beurteilung bzw. in der Einstufung als Umweltschaden. Dabei ergeben sich mehrere Schwierigkeiten. Zunächst stellt sich die Frage, wie der Referenzzustand rekonstruiert werden kann. Dafür kommen unmittelbar sichtbare Schäden in Form von toten oder kranken Individuen sowie beschädigter oder zerstörter Vegetation in Frage. Weiterhin können vorliegende Daten älteren Datums zum betroffenen Bereich ausgewertet werden. Ist beides nicht möglich, könnten Referenzdaten in angrenzenden Gebieten erhoben werden und als Analogieschluss auf den geschädigten Bereich übertragen werden. Des Weiteren ist der Zeitpunkt des Referenzzustandes zu diskutieren, bzw. was als Abweichung von der „natürlichen Fluktuation“ gelten kann. Natur ist nicht statisch, sondern entwickelt sich dynamisch. ${ }^{206}$ Diese Entwicklung ist von vielen

\footnotetext{
${ }^{203}$ Bundesnaturschutzgesetz: §19 Abs. 5 S. 1 BNatSchG, 07.08.2013a.

204 In seinem Aufsatz zur Naturverbundenheit von Naturvölkern verwendet Helbling den Begriff der Tragfähigkeit, „(...) wobei ,Tragfähigkeit' die maximale Bevölkerung bezeichnet, die in einem Territorium bei gegebener Technologie leben kann, ohne die langfristige Reproduktion der Ressourcen zu gefährden." Jürg Helbling, Ökologie und Politik in nicht-staatlichen Gesellschaften oder: Wie steht es mit der Naturverbundenheit sogenannter Naturvölker, in: Kölner Zeitschrift für Soziologie und Sozialpsychologie (1992) 44, S. 203-225, hier S. 203. Dieses Konzept ist insofern elegant, als es eine recht klare Grenze aufzeigt, ab wann die Umwelt nachhaltig, d. h. erheblich, geschädigt wird. Dieser Gedanke ist aufgrund der steten technischen Weiterentwicklungen zwar nicht direkt auf Industriegesellschaften übertragbar, nichtsdestotrotz könnte mit diesem Konzept gezeigt werden, ab welcher Bevölkerungsdichte die nächste Technologieweiterentwicklung erforderlich wäre.

${ }^{205}$ Bundesnaturschutzgesetz: §19 Abs. 5 Nr. 1 f. BNatSchG, 07.08.2013b.

206 Hierzu beschreibt Sieferle, dass es nach logischen Gesichtspunkten so etwas wie „Naturzerstörung“, sprich Umweltschaden, nicht geben kann: „Wenn ,Natur' selbst keine inhaltlich definierbare Ordnung oder Identität besitzt, sondern sich selbst immer wieder als ein Fluß von Ordnungszusammenhängen neu konstituiert, ist ,Naturzerstörung' nichts anderes als der Übergang von einem Ordnungszustand in einen anderen. [...] lediglich aus der (allerdings entscheidenden) Perspektive der menschlichen Kultur wird dies als ,Naturzerstörung' wahrgenommen." Sieferle, Sieferle 1993, S. 10-11 Auch Zirnstein weist darauf hin, dass Natur sich stetig und vor allem unregelmäßig wandelt: „In der Natur gibt es keine Beständigkeit, sondern Wandel. Der Wandel geschieht im allgemeinen allmählich, nur bisweilen rascher." Zirnstein, Zirnstein 1994, S. 15 Radkau beschreibt die Dynamik der Natur wie folgt: „Aus der Ökologie wissen wir seit langem, daß man sich unter ,Natur' keine stabile, in steter Harmonie befindliche organische Einheit vorstellen darf, sondern daß sich die Natur auch ohne menschliches Zutun ständig wandelt." Radkau, Radkau 2000, S. 33. Voland fasst zusammen: „Stabilität ist biologisch nicht normal." Eckart Voland, Welche Werte? Ethik, Anthropologie und Naturschutz, in: Philosophia
} 
Einflussfaktoren abhängig, wie z.B. der Regenhäufigkeit, die ihrerseits ebenso nicht notwendigerweise regelmäßig ablaufen. Ebenso kann z. B. ein Vulkanausbruch stark auf die Umwelt einwirken, die dadurch hervorgerufenen Fluktuationen wären jedoch als natürlich einzustufen. Es stellt sich also die Frage, wann der „richtige“ Zeitpunkt für den Vergleich mit dem jetzigen Zustand ist. ${ }^{207} \mathrm{Ob}$ es den „richtigen“ Zeitpunkt überhaupt gibt, ist allerdings umstritten, da „(...) es ökologisch keinen idealen Naturzustand gibt, der als normative Vorgabe dienen könnte.“208

Nicht zuletzt muss auch beschrieben werden, was eine „Beeinträchtigung der Funktion“ (USchadG, s. o.) bzw. eine „nachteilige Abweichung“ (BNatSchG, s.o.) eigentlich meint. Neben der unscharfen Formulierung (Beeinträchtigung, nachteilig), die Platz für Interpretation lässt, stellt sich die Frage, für wen oder was Nachteile oder Beeinträchtigungen entstehen. Der anthropozentrische Standpunkt des USchadG wurde weiter oben bereits aufgezeigt. Es liegt daher nahe, auch beim BNatSchG eine anthropozentrische Sichtweise zu unterstellen.

Abgesehen von den soeben beschriebenen Schwierigkeiten bei der praktischen Bestimmung eines Umweltschadens, stecken in diesen Bewertungen, wie der Begriff schon sagt, auch Werte. Wie also hängen Schäden und Werte zusammen?

Hauhs und Lange versuchen eine Trennung von Fakten und Werturteilen beim Schadensbegriff zu erreichen, indem sie drei Aspekte von Schäden unterscheiden: den Auslöser der realisierten Veränderung (Ursache), die Manifestation dieser Veränderung (Wirkung) und den Bewertungskontext zur Klassifikation einer Veränderung als Schaden (Wertung). ${ }^{209}$ Diese Unterteilung ist hilfreich bei der schrittweisen Einstufung eines Phänomens als Umweltschaden, denn wie in den oben zitierten Gesetzestexten gezeigt, arbeiten einige Ansätze auch mit der Art der Schadensursache. Auch die weitere Aufteilung in Wirkung und erst anschließende Wertung hilft, möglichst lange bei Fakten zu verweilen, bevor bewusst eine Bewertung vorgenommen wird. Für die Bewertung als Schaden ist es erforderlich, dass das Schutzgut vom Menschen als wertvoll eingestuft wird. „Als Schäden können (...) Zustandsveränderungen vielmehr nur betrachtet werden, insofern sie etwas

naturalis (2000) 37, S. 131-152, hier S. 137. Die Bestimmung des Referenzzustandes ist also ein wesentliches Problem bei der Bestimmung eines Umweltschadens. Mit Bezug auf Sieferle lässt sich aber zumindest die zentrale Bedeutung des anthropozentrischen Ansatzes feststellen.

${ }^{207}$ Eine ähnliche Fragestellung ergibt sich im Übrigen auch bei der Festlegung von Maßnahmen zur Schadensbeseitigung. „In allen Kontexten, in denen von ,Schäden' die Rede ist, wird eine aktuelle Struktur oder ein Verhalten mit einer für das betreffende System für möglich [Hervorh. i. O.] gehaltenen Struktur oder einem erwarteten Verhalten verglichen." Michael Hauhs/Holger Lange, Was ist ein ökologischer Schaden? Ein Ansatz für die Bestimmung virtueller Standards, in: Thomas Potthast (Hrsg.), Ökologische Schäden. Begriffliche, methodologische und ethische Aspekte 2004, S. 25-50, hier S. 31. Es wird also versucht zu bestimmen, wie sich die Umwelt ohne das Schadensereignis entwickelt hätte.

${ }^{208}$ Brüggemeier, Brüggemeier 2003, S. 4.

${ }^{209}$ Hauhs et al., S. 27. 
beeinträchtigen, was dem einen oder anderen und vielleicht sogar uns allen gemeinsam als ein Gut erscheint, das nach Möglichkeit bewahrt oder nach Kräften erreicht werden sollte.“210 Auch Skorupinski versteht unter einem Schaden eine Einbuße an einem Gut, das für jemanden einen Wert darstellt, und listet als Schadenskategorien auf: Personenschaden, Sachschaden, Handlungsfreiheit, Umweltqualität, Einkommensmöglichkeit, immaterielle Güter (z. B. Prestige, sozialer Friede, Ästhetik der Landschaft). ${ }^{211}$ Endres und Holm-Müller hingegen ordnen Umweltschäden drei verschiedenen Klassen zu: materielle Schäden, immaterielle Schäden und Gesundheitsschäden. Als Beispiele benennen sie u. a. für materielle Schäden Einkommens- und Vermögensminderungen und für immaterielle Schäden ästhetische Einbußen, Belästigungen durch Geruch o. Ä. und den Verlust an Erholungsmöglichkeiten. ${ }^{212}$ Nach Winiwarter gliedern sich Umweltschäden durch Zuordnung zu vier Paradigmen: ein toxikologisches, eines bezüglich Störungen von Ökosystemen, eines für Energie- und Materialverbrauch und schließlich eines für Gerechtigkeit und Moral. ${ }^{213}$

Doch bevor wir uns mit den Schadenskategorien respektive Wertekategorien befassen, lohnt noch ein kurzer Blick darauf, weshalb ein Schadensereignis besonders geeignet ist, Werte aufzuzeigen. „Es ist charakteristisch für Wertekonflikte oder Wertekrisen, daß die Werte besonders faßbar werden und daß der Historiker mehr Chancen hat, sie zu bemerken.“214 Dieses Konzept ist übertragbar auf Umweltschäden, da diese meistens einen Konflikt auslösen, sei es bezüglich der Einstufung als Schaden, der Ursache, des Schadensausmaßes oder auch der Maßnahmen zur Beseitigung des Schadens. Stollberg-Rilinger sieht den Konflikt ebenfalls als Chance, implizite Wertungen auf eine Ebene des diskursiven Begründens zu heben und so sichtbar zu machen. ${ }^{215}$ Uekötter sieht Krisen als besonders interessant für die Umweltgeschichte: „Insgesamt waren es weniger der Normalfall des Ressourcenkonsums als vielmehr die krisenhaften Umbrüche, die das Interesse der Umwelthistoriker provoziert haben." ${ }^{216}$ Kuckartz zeigt den Zusammenhang von Umweltschäden und Werten anhand der Risikoforschung: „Denn gerade Umweltthemen sind ungleich stärker als andere Themen mit Unsicherheit und Nicht-Wissen oder Nicht-Wissen-

\footnotetext{
${ }^{210}$ Heidrun Hesse, Umweltschäden und ökologisches Wissen - kleine Zwischenbetrachtung aus philosophischer Sicht, in: Thomas Potthast (Hrsg.), Ökologische Schäden. Begriffliche, methodologische und ethische Aspekte 2004, S. 51-56, hier S. 51.

211 Barbara Skorupinski, Gentechnik und ökologische Schäden als Gegenstand von Risikoforschung und partizipativer Technikfolgenabschätzung - Stand und Perspektiven, in: Thomas Potthast (Hrsg.), Ökologische Schäden. Begriffliche, methodologische und ethische Aspekte 2004, S. 169-188, hier S. 173.

212 Vgl. Alfred Endres/Karin Holm-Müller, Die Bewertung von Umweltschäden: Theorie und Praxis sozioökonomischer Verfahren, Stuttgart [u. a.], 1998, S. 24.

${ }^{213}$ Vgl. Winiwarter et al., Winiwarter et al. 2007, S. 120.

${ }^{214}$ Schmitt, Schmitt 2007, S. 31.

${ }^{215}$ Vgl. Stollberg-Rilinger, Stollberg-Rilinger 2007, S. 44-45.

${ }^{216}$ Uekötter, Uekötter 2007, S. 57.
} 
Können verbunden. Für Großrisiken ${ }^{217}$ wie den Klimawandel oder radioaktiven Müll gilt diese Unschärfe und Unsicherheit unseres Wissens noch in stärkerem Ausmaße. (...) Aus der Risikoforschung ist bekannt, dass die Einschätzung von Risiken ein in hohem Maße von Einstellungen und Wertvorstellungen bestimmtes Konstrukt ist. ${ }^{218}$ (...) Auch die Affinität zu postmaterialistischen Wertorientierungen (Toleranz, Solidarität, Kreativität, Eigeninitiative) lässt die Einschätzung des Gefahrenpotenzials (...) in die Höhe schnellen. “219 Hierzu passt die Entwicklung, dass die Angst vor Umweltschäden gestiegen ist. ${ }^{220}$

Um also den Wert zu bestimmen, der dem Phänomen, das als Schaden klassifiziert wird, zugrunde liegt, kann gefragt werden: Welches Schutzgut ist betroffen und welcher Nutzen wird gemindert? Oder mit anderen Worten: Warum ist dieses Phänomen unerwünscht?

Die Frage nach dem Nutzen stellt insbesondere die Ökologische Ökonomie, aus deren Sicht sich die Werte eines Menschen über seine Verzichtsbereitschaft zeigen. „Für Ökonomen gibt es nur eine ,Wertquelle': die Wertvorstellungen der Menschen. Diese werden so akzeptiert, wie sie sind (...). Sie zählen alle gleichermaßen bei der ökonomischen Bewertung, sofern sie eine Bedingung erfüllen: Sie müssen mit einer Verzichtsbereitschaft verbunden sein. Wenn jemand behauptet, dass inm etwas wertvoll sei, dass er aber nicht bereit sei, dafür irgendetwas zu ,opfern', in welchen Einheiten (Geld, Freizeit etc.) auch immer, berücksichtigen Ökonomen dies nicht. “221

Die einem Umweltschaden zugrundeliegenden Werte werden in der Ökologischen Ökonomie verschiedenen Kategorien zugeordnet. Hierfür werden üblicherweise englische Termini verwendet, welche sich in zwei Gruppen gliedern lassen. ${ }^{222}$ Wie in Abbildung 2 dargestellt,

\footnotetext{
${ }^{217}$ Hier ist zu unterscheiden zwischen kurzzeitigen Ereignissen mit unmittelbaren Folgen (z.B. Vulkanausbruch, Tankerunglück), üblicherweise als Katastrophe bezeichnet, und langfristigen Ereignissen, die nicht unmittelbar als Schadensereignis wahrgenommen werden und erst mittel- bis langfristig zu Schäden führen (z.B. Klimawandel). Uekötter weist hier auf eine Forschungslücke hin: Bislang gebe es keine Arbeit, die sich der theoretischen Klärung widmet, welche Faktoren ein Naturereignis zur Naturkatastrophe machen. Vgl. ebenda, S. 87.

218 „Die amerikanische Kulturtheorie nimmt an, dass die Menschen bei der Einschätzung von Risiken auf allgemeine, in ihnen tief verwurzelte Vorstellungen von Natur zurückgreifen." Udo Kuckartz, Trends im Umweltbewusstsein: Umweltgerechtigkeit, Lebensqualität und persönliches Engagement, Wiesbaden, 2006, S. 31.

${ }^{219}$ Ebenda, S. 28-29.

220 „Wurde 1985 auf eine offene Frage nach den drei am meisten ängstigenden Bereichen nur von $4 \%$ der Befragten die Angst vor Umweltzerstörung an erster Stelle genannt, so waren es 1988/89 bereits $18,2 \%$ und 1992 sogar $21,7 \%$ der Befragten. Damit stieg diese Angst vom siebten über den zweiten auf den ersten Rangplatz aller genannten Ängste auf (vgl. Boehnke/MacPherson 1993)." Günther, Günther 1997, S. 114.

${ }^{221}$ Krause et al., Krause et al. 2008, S. 294-295.

222 Interessanterweise wird in dieser Auflistung (Abbildung 2) der Wert „Gesundheit“ oder „Leben“ nicht genannt. „Einerseits besteht Einigkeit darüber, dass Gesundheit und Leben so konkurrenzlose Werte sind, dass ihr Verlust oder das Risiko ihres Verlustes durch nichts aufgewogen werden kann, schon gar nicht durch Geld. (...) Andererseits sind wir ständig gezwungen, diese ,unendlich wertvollen' Güter gegen andere abzuwägen. Wir gestehen damit implizit ein, dass ihr Wert doch endlich ist, lehnen aber eine explizite Wertung ab." Ebenda, S. 25.
} 
gibt es als erste Hauptgruppe die Use Values ${ }^{223}$. Diese nutzungsabhängigen Werte lassen sich wiederum in drei Untergruppen aufteilen: erstens die Direct Use Values als unmittelbar erfahrbare Werte wie z. B. der Wert als landwirtschaftliche Produktionsfläche. Zweitens gibt es die Indirect Use Values, bei denen sich ihr Wert nur indirekt zeigt, z. B. der Wert einer Fläche für die Klimaregulation, den Erosionsschutz oder als Überschwemmungsschutz. Hier zeigen sich teilweise Überschneidungen mit der dritten Gruppe, den Option Values oder Potenzialwerten. Diese Werte sind mögliche zukünftige Nutzungsoptionen, die aus heutiger Sicht vorteilhaft scheinen. Der Erosionsschutz beispielsweise kann sowohl in die Kategorie Indirect Use Value als auch in die Kategorie Option Value fallen, da der Nutzen zwar in beiden Fällen indirekter Natur ist, aber die Nutzung sowohl heute als auch in Zukunft stattfindet bzw. stattfinden wird.

1. Use Values (nutzungsabhängige Werte)
a. Direct Use Values
b. Indirect Use Values
C. Option Values

2. Non-Use Values (nicht-nutzungsabhängige Werte)
a. Existence Values (Existenzwerte)
b. Bequest Values (Vermächtniswerte)

\section{Abbildung 2: Wertekategorien (Ökologische Ökonomie)}

Die zweite Hauptgruppe bilden die Non-Use Values (nicht-nutzungsabhängige Werte). Diese untergliedern sich in die Existence Values und die Bequest Values. ${ }^{24}$ Unter den Existenzwerten sind Naturphänomene oder Arten zu verstehen, die als wertvoll betrachtet werden, wenngleich sie weder einen direkten noch einen indirekten (ökonomisierbaren) Nutzen aufweisen, so z. B. die Schönheit der Landschaft ${ }^{225}$ oder eine bestimmte Tierart, deren Nutzen (noch) nicht erkennbar ist, die aber aufgrund einer Eigenschaft oder einfach aufgrund der Tatsache ihrer Existenz als erhaltenswert eingestuft wird. Die Bequest Values

\footnotetext{
223 „Natur zählt ökonomisch nur, sofern sie zur Wohlfahrt beiträgt. Wohlfahrt bzw. Nutzen wird als Funktion von Konsum aufgefasst, wobei der Konsumbegriff häufig auf Warenkonsum reduziert wird. Dadurch kann Naturgenuss in seinen Wohlfahrtswirkungen kaum noch adäquat erfasst werden. Die Natur war und ist der ,blinde Fleck' der modernen Ökonomik." Ott, Ott 2010, S. 33-34. Hier ist Ott zu widersprechen, da die Ökologische Ökonomie durchaus auch den Aspekt des ästhetischen Naturgenusses zu berücksichtigen versucht bzw. durch die Verwendung der Non-use Values eben gerade die nicht-nutzungsabhängigen Werte abbilden möchte (siehe auch Fußnote 226).

${ }^{224}$ Für die Gruppe der Non-Use Values haben Forscher sehr unterschiedliche Begriffe gefunden, um deren Inhalte zu beschreiben. Die oben genannten scheinen sich am besten durchgesetzt zu haben. Crowards listet die folgenden Begriffe auf, die Non-Use Values beschreiben: „preservation values, intrinsic values, existence values, passive use values, intangibles, off site use values, non-user values" Tom Crowards, Nonuse Values and the Environment: Economic and Ethical Motivations, in: Environmental Values 6 (1997), S. 143-167, hier S. 147.

${ }^{225}$ Vgl. Breckling et al., S. 9.
} 
oder Vermächtniswerte grenzen sich zu den Option Values dadurch ab, dass offen ist, ob eine zukünftige Nutzung möglich oder erwünscht ist, aber das Phänomen oder die Art trotz (noch) nicht erkennbarem Nutzen für künftige Generationen erhalten werden soll. Da die Gruppe der Non-Use Values nicht ökonomisierbare Werte beinhaltet, ist deren Existenz in der Ökonomie strittig. ${ }^{226}$ Aus umweltgeschichtlicher Sicht erscheint die Existenz solcher Werte allerdings weniger strittig. Im textanalytischen Teil der vorliegenden Arbeit finden sich solche Wertekategorien z. B. in den Werten Nachhaltigkeit, Unberührte Natur oder Schönheit der Natur.

An dieser Stelle zeigen sich zum einen die Schwierigkeiten eines interdisziplinären Arbeitsfeldes wie dem der Umweltgeschichte und zum anderen die anthropozentrische Herangehensweise der Ökologischen Ökonomie. Aber nicht nur in der Ökologischen Ökonomie herrscht dieser Ansatz vor, auch in der Umweltgeschichte ist ein anthropozentrischer Ansatz nahezu unvermeidlich.

\subsubsection{Wertearten und der anthropozentrische Ansatz}

Im folgenden Abschnitt geht es um die verschiedenen Arten von Werten und darum, weshalb der anthropozentrische Ansatz für die Umweltgeschichte so zentral ist. Ergänzend werden Verbindungen zu der Wertetheorie von Schwartz aufgezeigt.

Für Hughes hat Umweltgeschichte zwangsläufig einen anthropozentrischen Ansatz: „Environmental history is not simply the history of the environment. The human side of the relationship is always included. (...) This means that environmental history inevitably has a human-centered approach, although environmental historians are keenly aware that humans are part of nature, dependent on ecosystems, and not entirely in control of their own destiny."227 Die Behauptung, dass umweltgeschichtliche Fragestellungen immer die menschliche Perspektive beinhalten, wirft die Frage nach dem Warum auf. Nun könnte man entgegnen, dass Umweltgeschichte sich per Definition (vgl. Abschnitt 2.1.1) mit den Wechselwirkungen zwischen Mensch und Natur befasst und demnach die menschliche Seite dieser Beziehung unvermeidlich Teil der Forschung ist. Allerdings geht Hughes in seiner Ausführung einen Schritt weiter, indem er von einem „human-centered approach“ spricht. Hier verlassen wir die (theoretisch) objektive Ebene der Wechselwirkungsbeschreibung zwischen Mensch und Natur und gelangen zur tiefergehenden Frage der Sichtweise, mit der ein Phänomen betrachtet wird. Proctor stellt hierzu fest, dass es zwar eine Natur gibt, wir aber zur Beschreibung dieser Natur auf die menschliche Wahrnehmung derselben

\footnotetext{
${ }^{226}$ Vgl. ebenda, S. 9.

${ }^{227}$ Hughes, Hughes 2006, S. 4.
} 
angewiesen sind. „There certainly is a nature 'out there', but we cannot say anything more about it without relying on human modes of perception (...) - in short, when we speak of nature we speak of culture as well, of the meanings we attribute to nature. ${ }^{\text {"22 }}$ Die Bedeutungen, von denen Proctor hier schreibt, könnten auch als Werte bezeichnet werden. Diesbezüglich stellt Voland richtigerweise fest: „Werte sind in der Natur nicht vorfindlich (...), Werte werden selbstverständlich zugewiesen und zwar ausschließlich von moralischen Subjekten.“"229 Damit ist die menschliche Wahrnehmung, respektive Bewertung der Natur in der Tat unvermeidlich und führt uns zur nächsten Frage: Welche Arten von Wert werden der Natur zugeordnet?

Die Naturethik unterscheidet drei Hauptgruppen von Wertarten, wie in Abbildung 3 dargestellt. Erstens den anthropozentrischen Wert, der sich weiter unterteilen lässt in nutzungsabhängige und nicht-nutzungsabhängige Werte (vgl. hierzu Abbildung 2). Zweitens den physiozentrischen Wert, der auf eine Natur ohne Bezug zum Menschen abstellt und sich untergliedert in pathozentrische Werte, d. h. alles fühlende Leben betreffend, und in biozentrische Werte, die alles Lebende betreffen. Anthropozentrische Werte (inhärente Werte ${ }^{230}$ ) beruhen im Gegensatz zu physiozentrischen (intrinsischen Werten) auf der Beziehung zwischen Mensch und Natur. ${ }^{231}$ Drittens den ökozentrischen Wert, der belebte sowie unbelebte Natur einschließt und insbesondere den Wert des „Ganzen“ im Gegensatz zu einzelnen Lebewesen hervorhebt.

- Anthropozentrisch (inhärent)

○ Nutzungsabhängig

○ Nicht-nutzungsabhängig

- Physiozentrisch / Nicht-anthropozentrisch (intrinsisch)

- Pathozentrisch

○ Biozentrisch

- Ökozentrisch

\section{Abbildung 3: Wertekategorien (Naturethik)}

Der anthropozentrische Ansatz kann sich auf zwei verschiedenen Ebenen auf das menschliche Leben beziehen. Zum einen auf einer epistemischen Ebene: Wertungen erfolgen durch den Menschen und können folglich nur durch inn verstanden werden. Dies

\footnotetext{
${ }^{228}$ Proctor, S. 229.

${ }^{229}$ Voland, Voland 2000, S. 137.

230 „Das Konzept des 'inhärenten Wertes' geht auf C.I. Lewis zurück, der die Dinge als ,inhärent' wertvoll bezeichnet, die das Potential haben, zu einer wertvollen Erfahrung beizutragen." Lewis, 1962, S. 432ff, An analysis of knowledge and valuation, zitiert nach Krause et al., Krause et al. 2008, S. 303.

${ }^{231}$ Vgl. Potthast, Potthast 2000, S. 119.
} 
bedeutet aber keineswegs, dass der Mensch höher bewertet wird als andere Naturelemente. Zum anderen auf einer moralischen Ebene: hier ist der Mensch das wichtigste Element, alle Wertungen beziehen sich darauf, ob etwas gut für den Menschen ist. Dies können neben biologischen Grundbedürfnissen auch ästhetische oder emotionale Bedürfnisse sein. Eine anthropozentrisch ausgerichtete Ethik muss jedoch nicht zwangsläufig bedeuten, dass Umwelt nur als Ressource (instrumentell) gesehen wird; vergleiche hierzu das Konzept der nicht-nutzungsabhängigen Werte (Kapitel 2.2.4).

Was bedeutet das nun für den Anthropozentrismus im Zusammenhang mit Umweltgeschichte? Die oben beschriebene moralische Ebene dürfte einen großen Teil der umweltgeschichtlichen Fragestellungen begründen. Hierbei nehmen die nicht-nutzungsabhängigen Werte zwar eine besondere Stellung ein, aber letztlich geht es auch bei diesen Werten darum, ob etwas von Vorteil für den Menschen ist. Allerdings existieren durchaus auch Fragestellungen mit physiozentrischen Wertansätzen. Ein Beispiel ist das amerikanische Konzept von „wilderness“, bei dem der sich selbst überlassenen Natur ein Selbstwert unabhängig vom Menschen zugeordnet wird. Genau hierin liegt nun aber auch ein Widerspruch. Denn, wie oben ausgeführt, in der Natur gibt es keine Werte. Erst der Mensch ordnet diese Werte zu, wobei es hierbei nachrangig ist nach welchen Kriterien er dies tut. Gleiches gilt für den ökozentrischen Ansatz. Insofern kann es also genaugenommen nichtanthropozentrische Werte meines Erachtens nach nicht geben. Lässt man jedoch den Umstand außer Acht, dass nur der Mensch Werte zuordnet und akzeptiert das Konzept der intrinsischen Werte, führt dies zu einer sinnvollen Untergliederung wie in Abbildung 3 dargestellt. Wenn Uekötter also feststellt: „Inzwischen scheint es unter den Forschern einen Konsens zu geben, dass die Fragestellungen der Umweltgeschichte unvermeidlich anthropozentrisch begründet sind.“232, ist inm zunächst einmal zuzustimmen und zudem sollte diese Aussage meines Erachtens im Sinne einer möglichst weiten Auslegung des anthropozentrischen Begriffs interpretiert werden, also auch physiozentrische und ökozentrische Ansätze einschließen.

Dass diese umweltbezogenen Wertearten nicht nur für die Umweltgeschichte bedeutsam sind, sondern dass die Werthaltung gegenüber unserer Umwelt eine grundlegende und weltweit nachweisbare Dimension menschlichen Verhaltens ist, zeigten die Forschungen von Schwartz. Er untersuchte die Frage, ob es universelle Werte gibt und führte eine aufwändige Studie durch, die seine Wertetheorie bestätigte. Seine Ergebnisse zeigten, dass die Wertvorstellungen von Menschen überall auf der Welt mit drei grundlegenden Problemen zu tun haben: dem Überleben als Individuum, der Koordination der sozialen Interaktion und dem

\footnotetext{
${ }^{232}$ Uekötter, Uekötter 2007, S. 3.
} 
Überleben von Gruppen. ${ }^{233}$ Demnach gibt es länderübergreifend drei bipolare Grunddimensionen ${ }^{234}$ dieser Problemstellungen:

- Eingebettetsein vs. Autonomie (Conservatism vs. Autonomy),

- Egalitarismus vs. Hierarchie (Egalitarian Commitment vs. Hierachy),

- Umweltbeherrschung vs. Harmonie (Mastery vs. Harmony).

Die dritte Paarung thematisiert den Willen zur Umweltbeherrschung und der Gestaltung und Kontrolle der Lebensbedingungen sowie den entgegengesetzten Ansatz, die (Um)Welt in ihrer ursprünglichen Form zu akzeptieren bzw. die friedliche Einheit mit der Natur. Mit den sich daraus ergebenden Wechselwirkungen zwischen Mensch und Natur befasst sich die Umweltgeschichte.

Die Frage, ob es universelle Werte gibt, haben sich auch andere Forscher gestellt. Stern kam zu dem Schluss, dass es zwei übergeschichtliche, existenzielle Werte für den Menschen gibt: Leben und Gesundheit (vgl. Fußnote 170, S. 37). Um beides zu sichern, ist die Erhaltung günstiger Lebensbedingungen für den Menschen wichtig. Hier ist der Ursprung des Wertes „Umweltschutz“ zu sehen, wobei im Lauf der Zeit dem Umweltschutz weitere Themen zugeordnet wurden wie z. B. die Einführung von Nationalparks. Radkau sieht in der dauerhaften Erhaltung von günstigen Lebensumständen bzw. der dauerhaften Versorgung mit Nahrung gar einen absoluten Maßstab zur Bewertung einer Kultur: „Einen absoluten Maßstab für die ökonomisch-ökologische Bewertung einer Kultur bietet das Kriterium, ob sie die Ernährung ihrer Bevölkerung auf nachhaltige Weise sichert." ${ }^{235}$ Littig formuliert als Konsequenz kollektives Umweltbewusstsein und entsprechendes Verhalten: „Die drohende Zerstörung der existenziellen natürlichen Lebensbedingungen des Menschen durch den Menschen mobilisiert eine kritische Haltung gegenüber einem zerstörerischen MenschNatur-Verhältnis. Das Mensch-Natur-Verhältnis wird moralisiert. ,Umweltbewußtsein' als kollektiv gültiges Deutungsmuster ist Ausdruck dieser Haltung und verlangt nach entsprechenden Verhaltensänderungen“. ${ }^{236}$ Was aber beinhaltet Umweltbewusstsein? Littig führt weiter aus: „In der neueren sozialwissenschaftlichen (...) Umweltforschung ${ }^{237}$ wird ,Umweltbewußtsein' als mehrdimensionales, allein kognitive Dimensionen berücksichtigen-

\footnotetext{
${ }^{233}$ Vgl. Klaus Jonas/Wolfgang Stroebe/Miles Hewstone/Carmen Lebherz/Matthias Reiss, Sozialpsychologie: Eine Einführung, Heidelberg, 2007, 5., vollst. überarb. Aufl, S. 572.

${ }^{234}$ Vgl. ebenda, S. 573.

${ }^{235}$ Radkau, Radkau 2000, S. 39.

236 Beate Littig, Die Bedeutung von Umweltbewußtsein im Alltag: Oder: was tun wir eigentlich, wenn wir umweltbewußt sind?, Frankfurt am Main, 1995, S. 142.

237 „Die Umweltengagierten (..) neigen in ihren Wertorientierungen stark zu postmaterialistischen Werten: So stehen bei ihnen Eigeninitiative, Solidarität, Kreativität und Toleranz signifikant höher im Kurs. In Bezug auf traditionelle Werte, wie zum Beispiel Disziplin, Pflichtbewusstsein, Fleiß, Heimatverbundenheit, Leistungsbewusstsein oder Höflichkeit unterscheiden sie sich nicht von den anderen Befragten." Kuckartz, Kuckartz 2006, S. 42.
} 
des Konstrukt gefaßt. (...) Elemente dieses Konstrukts sind: umweltbezogene Werthaltungen, umweltrelevantes Wissen, umweltbezogene Einstellungen, Umwelterleben und betroffenheit, umweltorientierte Handlungsbereitschaft.“"238 Urban ergänzt hierzu: „Umweltbezogene Einstellungen machen den Kernbereich des Umweltbewußtseins aus.“239 An dieser Stelle muss eine Unterscheidung zwischen Werten und Einstellungen erfolgen. Werte sind allgemeiner, Einstellungen spezifischer auf einen bestimmten Sachverhalt oder Gegenstand bezogen und damit auch handlungsnäher. „Wertorientierungen sind abstrakter als Einstellungshaltungen (...) und liefern deshalb auch Bewertungsmaßstäbe, die wesentlich handlungsferner formuliert sind als die evaluativen Inhalte von Einstellungsmustern.“240 Zumindest die im Umweltbewusstsein enthaltenen umweltbezogenen Werthaltungen zielen auf den dauerhaften Erhalt von günstigen Lebensbedingungen für den Menschen. Dafür werden verschiedene Werthaltungen gegenüber diversen Phänomenen herausgebildet. Der existenzielle Wert "Leben“ könnte dafür z. B. weiter untergliedert werden in Wohnraum, Wasser, Nahrung und Luft. Durch diese Gliederung entstehen greifbare Einzelwerte, die genauer zu beschreiben sind und auch gegeneinander abgewogen werden können.

In der Textanalyse dieser Arbeit werden die Wertekategorien hingegen induktiv erarbeitet, womit wir noch einmal auf die eingangs diskutierten Wertarten zurückkommen. In den Textanalysen sollen sämtliche enthaltene Werte erfasst werden. Die Zuordnung, ob anthropozentrisch oder nicht, erfolgt erst anschließend. Aber es gibt auch noch weitere Differenzierungen, Schweidler unterscheidet hier drei verschiedene Wertbegriffe: „Der ökonomische Wertbegriff ist prinzipiell in Bezug auf Nutzen, der soziologische in Bezug auf Funktion, der ethische in Bezug auf Wahrheit definiert.“241 Die Größe des ökonomischen Wertes wird durch Angebot und Nachfrage der Marktteilnehmer bestimmt. Ökonomische Werte können verglichen und gegeneinander aufgewogen werden. Sozio-logische Werte sind gesellschaftlich vermittelte Einstellungen, die im zeitlichen Verlauf wandelbar sind und in Abhängigkeit zur jeweiligen Kultur stehen. ${ }^{242} \mathrm{Da}$ es heute üblich ist, zwischen Wert (ökonomisch) und Werten (ethisch) zu unterscheiden, erscheint es wichtig darauf hinzuweisen, dass in der Textanalyse keine Wertearten nach diesen Kriterien ausgeschlossen werden, um ein möglichst komplettes Bild der enthaltenen Werte zu bekommen.

\footnotetext{
${ }^{238}$ Littig, Littig 1995, S. 55.

${ }^{239}$ D. Urban, Was ist Umweltbewußtsein?, in: Zeitschrift für Soziologie (1986) 15, S. 363-377, hier S. 365.

${ }^{240}$ Ebenda, S. 365.

${ }^{241}$ Walter Schweidler, Einführung, in: ders., Werte im 21. Jahrhundert, Baden-Baden, 1. Aufl, S. 7-18, hier S. 10.

${ }^{242}$ Vgl. ebenda, S. 10.
} 


\section{Methodik der Textanalyse}

\subsection{Methode: Qualitative Inhaltsanalyse}

Es stellt sich die Frage, mit welcher Methode sich implizite Wertvorstellungen in umwelthistorischen Texten analysieren lassen. Als solche werden hier wissenschaftliche Texte betrachtet, die sich mit Themen der Umweltgeschichte auseinandersetzen. Dies schließt Abhandlungen über Umweltgeschichte (Metaebene) aus. Implizite Wertvorstellungen liegen nicht offen im Text vor, sondern zeigen sich nur indirekt über den Sprachgebrauch. Es ist also primär eine qualitative Methode anzuwenden, da die Fragestellung auch eine Interpretation ${ }^{243}$ der zu untersuchenden Textinhalte erfordert.

Zunächst könnte sich das Verfahren zur Diskursanalyse anbieten. In der Geschichtswissenschaft befasst sich Diskursanalyse u. a. mit den Regeln der Verarbeitung und Darstellung von historischen Geschehnissen. Allerdings steht bei dieser Herangehensweise zumeist eine soziolinguistische Betrachtung im Vordergrund. „Diskursanalyse ist demgegenüber ein Verfahren der analytischen Rekonstruktion gesellschaftlicher Regelmäßigkeiten, ohne deren Ordnungsstrukturen aus einer vorgängig formulierten Gesellschaftstheorie zu beziehen (...). ${ }^{\text {“244 }}$ Ziel dieser Arbeit ist jedoch weder die semantische Analyse des gesamten Textes, noch die Analyse einer soziologischen Interaktion. Vielmehr soll ein spezieller Aspekt innerhalb eines Textes untersucht werden, der zudem nicht direkt aus dem Textmaterial abzuleiten ist. Hierfür ist die Inhaltsanalyse als etablierte Methode der qualitativen Sozialforschung besser geeignet, da sie als Interpretationsmethode Zugang zu subjektiven Sichtweisen ermöglicht. „Dass Realität (...) über (...) individuelle Interpretationsleistungen vermittelt $(\ldots)$ wird, sind Hintergrundannahmen unterschiedlicher qualitativer Forschungsansätze.“245 Mayring weist darauf hin, dass eine Inhaltsanalyse nicht nur die oberflächlichen Inhalte berücksichtigt: „Qualitative Inhaltsanalyse ist immer ein Verstehensprozeß [Hervorh. i. O.] von vielschichtigen Sinnstrukturen im Material. Die Analyse darf nicht bei dem manifesten Oberflächeninhalt steckenbleiben, sie muß auch auf latente Sinngehalte [Hervorh. i. O.] abzielen.“246 Die Inhaltsanalyse unterstellt allgemein, dass Menschen ihre Ansichten und Einstellungen, also auch ihre Wertvorstellungen, in dem zum Ausdruck bringen, was sie sagen oder schreiben. Wenngleich Wissenschaftler einen möglichst hohen Grad an Objektivität anstreben, so gilt diese Annahme auch für diesen Personenkreis. Dieser

\footnotetext{
243 „Das mühsame Geschäft der Interpretation ist nicht anders als durch Einzelfallanalysen zu leisten.“ Soeffner et al., Soeffner et al. 2004, S. 553.

${ }^{244}$ Reiner Keller, Handbuch Sozialwissenschaftliche Diskursanalyse, Wiesbaden, 2008, 3., aktualisierte und erw. Aufl, S. 233.

${ }^{245}$ Uwe Flick/Ernst von Kardorff/Ines Steinke, Was ist qualitative Forschung? Einleitung und Überblick, in: ders., Qualitative Forschung. Ein Handbuch, Reinbek bei Hamburg, S. 13-29, hier S. 21.

${ }^{246}$ Philipp Mayring, Qualitative Inhaltsanalyse: Grundlagen und Techniken, Weinheim, 2008, 10. Aufl., S. 29.
} 
Aspekt der Inhaltsanalyse ist hervorzuheben, da es in der vorliegenden Arbeit auch um implizite Werte geht, also Werte, die, bewusst oder unbewusst, den Text beeinflusst haben, aber nicht direkt im Text in Erscheinung treten. Gegenstand moderner Inhaltsanalyse sind auch latente Sinngehalte. ${ }^{247}$

Qualitative Analyse- und Interpretationsverfahren sind in starkem Maß kontextorientiert. ${ }^{248}$ Erst die Betrachtung und Interpretation im Kontext eröffnet die Sicht auf implizit enthaltene Wertvorstellungen (siehe Abschnitt 3.2.2). Die Inhaltsanalyse zeichnet sich durch ihre theoriegeleitete, regelgeleitete und an Kategorien orientierte Systematik aus. ${ }^{249}$ Auch der Anspruch dieser Arbeit auf intersubjektive Nachvollziehbarkeit als Gütekriterium spricht demnach für die qualitative Inhaltsanalyse. Wenngleich Kategorien verwendet werden, um die einzelnen Fälle darunter zu subsumieren, ist durch die induktive Kategorienbildung die Entdeckungslogik, oder das Prinzip der Offenheit, gewährleistet. In diesem Punkt ist Rosenthal zu widersprechen, die im subsumtionslogischen Verfahren der Inhaltsanalyse einen Widerspruch zur rekonstruktiven Arbeitsweise der interpretativen Textanalyse sieht. ${ }^{250}$

In der Umweltgeschichte werden in der Natur zu beobachtende Phänomene beschrieben sowie die Ursachen und Auswirkungen dieser Phänomene unter besonderer Berücksichtigung der Wechselwirkung zwischen Mensch und Umwelt analysiert. Die hierbei zugrundeliegenden impliziten Werte können als theoretische Begriffe nicht direkt beobachtet werden. Daher wird stattdessen ein Indikator verwendet, der mit dem zu beobachtenden Sachverhalt korrespondiert. Als Indikator soll die Verwendung des Wortes „Umweltschaden“ im zu analysierenden Text dienen (siehe Abschnitt 3.2.1). Als Schaden lassen sich die unerwünschten Folgen eines Ereignisses bezeichnen. Die Gründe für diese Unerwünschtheit finden sich in den zugrundeliegenden Werten. Wie der Gebrauch des Plural bereits andeutet, kann der Indikator auf verschiedene Ausprägungen (im Folgenden als Kategorien bezeichnet) hindeuten. Um weiter zu differenzieren muss die Verwendung des Indikators im Kontext interpretiert werden (siehe Abschnitt 3.2.2).

Implizite Werte sind, wie oben gezeigt wurde, anhand von Indikatoren zu ermitteln. Darüber hinaus gibt es in Texten aber noch weitere Indizien für implizite Werthaltungen, die allerdings nicht so klar zu formulieren und abzugrenzen sind wie eine konkrete Suche nach Stichwörtern (Indikatoren):

\footnotetext{
247 Vgl. Philipp Mayring, Qualitative Inhaltsanalyse, in: Uwe Flick/Ernst von Kardorff/Ines Steinke (Hrsg.), Qualitative Forschung. Ein Handbuch, Reinbek bei Hamburg 2009, S. 468-474, hier S. 469.

${ }^{248}$ Vgl. Flick et al., S. 23.

${ }^{249}$ Vgl. Mayring, S. 471.

${ }^{250}$ Rosenthal, Rosenthal 2005, S. 55.
} 
- zwischen den Zeilen“,

- Art der Formulierung,

- Auswahl der präsentierten Fakten.

Das erste Indiz, „Zwischen den Zeilen“, ist besonders schwer zu fassen, denn hier gibt es keinen konkreten Punkt oder ein bestimmtes Wort, das auf einen Wert hinweist. Vielmehr erzeugt der Text beim Leser ein eher diffuses Gefühl ${ }^{251}$, dass der Autor eine bestimmte Werthaltung hat, diese aber nicht direkt zum Ausdruck bringt. Zumeist wird es aber einen Hinweis geben und zwar in der Art der Formulierung. Damit ist gemeint, dass es einen Unterschied macht, wie ein Phänomen beschrieben wird. Dies lässt sich mit einem einfachen Beispiel verdeutlichen: Analysiert ein Text die Folgen von Windkraftanlagen, kann der Autor den Anblick eines Windparks auf verschiedene Arten beschreiben. Er kann eine wertfreie Feststellung treffen: „Am Horizont sind 10 Windräder zu sehen.“ Er kann aber auch eine wertende Formulierung wählen: „Die Windräder bewirken eine Verspargelung der Landschaft." Diese umgangssprachliche Formulierung bringt eine bestimmte Einstellung zum Ausdruck. Das dritte Indiz ist die Auswahl der präsentierten Fakten. Denn nicht nur das, was letztlich im Text erscheint, sagt etwas über die Werthaltungen des Autors aus, sondern auch das, was nicht im Text erscheint. Dies gilt selbst dann, wenn der Text so wertneutral wie möglich formuliert ist. Damit ist gemeint, dass es etwas bedeutet, z. B. die Anzahl der menschlichen Opfer zu nennen, aber nicht, welche bzw. wie viele Tiere starben oder verletzt wurden oder ob z. B. Wald zerstört wurde. Das Weglassen von bestimmten Tatsachen deutet in diesem Beispiel auf eine anthropozentrische Haltung hin. Aus der Art des Phänomens lässt sich auf die möglichen Schäden schließen. So dürfte z. B. ein Vulkanausbruch sowohl menschliche Verluste als auch Verluste in Fauna und Flora bewirken. Welche Verluste in den Text eingehen bzw. welche Auswirkungen (auch hier: Auswirkungen für wen oder was) dargestellt werden, zeigt die Prioritäten des Autors. Als Einschränkung für dieses dritte Indiz muss allerdings der Titel des Textes genannt werden: Wird hier bereits darauf hingewiesen, dass das analysierte Phänomen nur unter einem speziellen Aspekt betrachtet wird muss dies bei den Überlegungen zu impliziten Werthaltungen berücksichtigt werden. Die Interpretation anhand der eben beschriebenen Indizien soll allerdings nur ergänzend zu der Analyse über Indikatoren erfolgen, da die methodische und die intersubjektive Nachvollziehbarkeit dort wesentlich besser sind.

Die identifizierten Werte werden anschließend Wertekategorien zugeordnet. Diese Kategorien werden nicht vorab definiert, sondern induktiv gebildet (siehe Abschnitt 3.2.2).

${ }^{251}$ Umgangssprachlich hört man dazu auch: „da schwingt etwas mit“. Dieser Ausspruch fasst das diffuse Gefühl zusammen, dass der Autor eben nicht nur Fakten präsentiert, sondern auch eine Meinung zum Ausdruck bringt. 
Nach Abschluss dieser qualitativen Analyse werden die erarbeiteten Daten quantitativ ausgewertet. Neben der grundsätzlichen Frage, welche Werte in umwelthistorischen Texten dominieren, kann so geprüft werden ob z. B. das Umfeld (Universität) mit einer Häufung bestimmter Werte korreliert.

Zur Auswertung der Textanalysen sollen die Texte bzw. deren Inhalte in einer Matrix verortet werden, die einerseits den geographischen Wirkungskreis der diskutierten Phänomene erfasst und andererseits das zeitliche Element abbildet.

Diese Arbeit orientiert sich am Ablaufmodell nach Mayring für die strukturierende qualitative Inhaltsanalyse in Verbindung mit der ebenfalls von Mayring vorgeschlagenen Abfolge für die induktive Kategorienbildung. Abbildung 4 zeigt den Ablauf der Textanalyse.

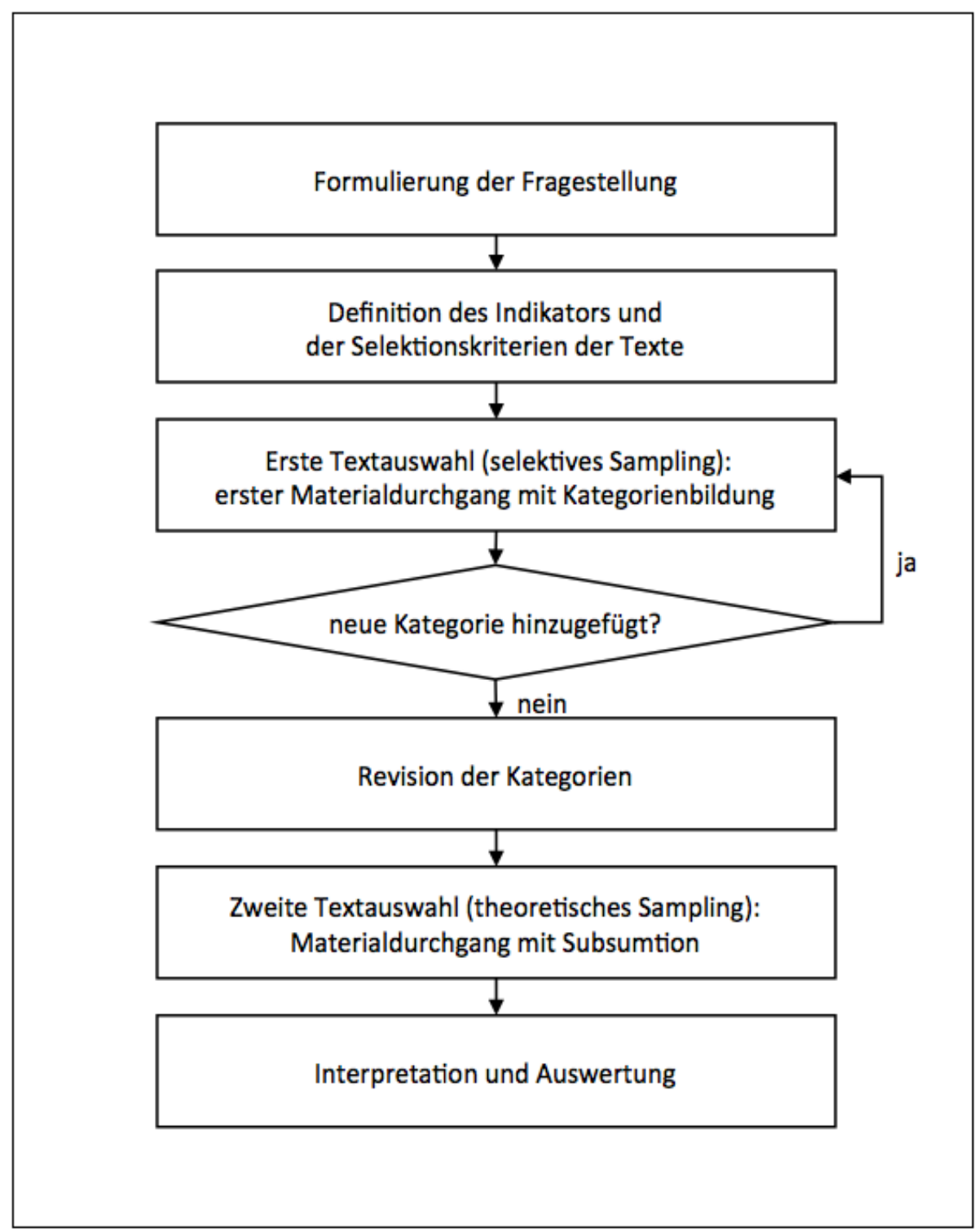

Abbildung 4: Ablaufdiagramm der qualitativen Inhaltsanalyse dieser Arbeit ${ }^{252}$

$\overline{252}$ Eigene Darstellung in Anlehnung an Mayring 2002, S. 116. 
Die Textanalyse gliedert sich in zwei Abschnitte: den ersten Materialdurchgang, basierend auf selektivem Sampling, und den zweiten Materialdurchgang, der auf theoretischem Sampling basiert. Diese Schritte werden im folgenden Abschnitt detailliert beschrieben. Die erste Materialauswahl wird erst beendet, wenn keine neuen Kategorien mehr hinzugefügt werden. Nach Abschluss dieses Analyseschritts erfolgt die Revision der erarbeiteten Kategorien. Wie in Abbildung 4 dargestellt, schließt sich an die Überarbeitung der Kategorien dann der zweite Materialdurchgang an, gefolgt von Interpretation und Auswertung der Analyseergebnisse.

Die Texte werden unter der Verwendung von MAXQDA (Software für qualitative Datenanalyse) analysiert. Die computergestützte Analyse begünstigt ein systematisches Vorgehen, erleichtert die Zugänglichkeit bzw. das Auffinden z. B. von Notizen oder Bewertungen und gestattet eine effiziente Auswertung der gewonnenen Daten. Nicht zuletzt ist die Verwendung eines solchen Programms für die Nachvollziehbarkeit vorteilhaft, da sämtliche Auswertungsschritte detailliert dokumentiert werden können und anschließend in elektronischer Form vorliegen.

Diese Nachvollziehbarkeit ist auch hinsichtlich der Gütekriterien für qualitative Forschung von Bedeutung. Der Forschungsablauf soll intersubjektiv nachvollziehbar sein. Dies wird durch die Markierung der Fundstellen sowohl von Indikatoren als auch von Werten, Beschreibungen zu den verschiedenen Wertekategorien (sogenannte Code-Memos) und der möglichst detaillierten Schilderung der Forschungsschritte gewährleistet. Gleiches gilt für die Reliabilität; die Beschreibungen bzw. Definitionen der Wertekategorien und der Indikatoren stärken die Zuverlässigkeit der Ergebnisse. Auch die induktive Kategorienbildung trägt zur Stabilität der Ergebnisse bei. Bezüglich der Validität sind systematische Fehler und falsche bzw. falsch interpretierte Indikatoren zu vermeiden. Dies wird durch eine Beschreibung der Indikatoren erreicht. Schließlich gibt es noch das Gütekriterium der Repräsentativität. Wie in der Einleitung beschrieben ist das Ziel der Untersuchung eine deskriptive Aussage zu den in den untersuchten Texten enthaltenen Werten. Ein möglicher Rückschluss auf die Grundgesamtheit wird durch die Stichprobenzusammensetzung angestrebt.

\subsection{Operationalisierung der Textanalyse}

\subsubsection{Der Indikator „Umweltschaden“}

Nachdem im Abschnitt 2.2.4 der hier verwendete Begriff „Umweltschaden“ definiert und erörtert wurde, geht es nun um die praktische Anwendung in der Textanalyse. Neben dem Wort „Umweltschaden“ werden die Texte auch nach Abwandlungen dieses Begriffs, nach 
Synonymen und inhaltlich ähnlichen Begriffen ${ }^{253}$ durchsucht, um sicherzustellen, dass alle relevanten Passagen erfasst werden. Zur besseren Übersichtlichkeit werden die Indikatoren wie folgt gruppiert:

- Umweltschaden: ausschließlich die Worte „Umweltschaden“ und „ökologischer Schaden“. Im Englischen „environmental damage“ und „ecological damage“.

- Schaden: der Begriff „Schaden“ und Ableitungen wie schädlich, schädigend, Beschädigung. Im Englischen „damage“, „pollution“ und „environmental decline“ sowie entsprechende Ableitungen.

- Katastrophe: das Wort „Umweltkatastrophe“ oder "Katastrophe” und Ableitungen davon. Im Englischen "catastrophe“, „disaster" und "calamity“ sowie entsprechende Ableitungen.

- Umweltzerstörung: das Wort „Umweltzerstörung“ oder „Zerstörung“ und entsprechende Ableitungen. Im Englischen „destruction“ und „degradation" sowie Ableitungen.

- Umweltbedrohung: der Begriff „Umweltbedrohung“, „Problem“ oder „Bedrohung“ und Ableitungen. Im Englischen „threat“ und „problem“ sowie entsprechende Ableitungen.

Wird ein Stichwort gefunden, wird es zunächst im unmittelbaren Textzusammenhang gelesen. Falls es mit der Konnotation „Umweltschaden“ verwendet wird, wird die Fundstelle markiert und der Text wird hinsichtlich der Werte analysiert. Werden beim Durcharbeiten der Texte weitere Indikatoren gefunden, die durch die oben beschriebene Suche nicht abgedeckt waren oder nicht erfasst wurden ${ }^{254}$, werden diese ebenfalls markiert. Die möglichst vollständige Erfassung aller Fundstellen von Indikatoren könnte für die abschließende Auswertung der Analyseergebnisse Relevanz haben. Nicht einbezogen werden Indikatoren, die in Fuß- oder Endnoten enthalten sind sowie in Literaturverzeichnissen.

Alle Indikatoren beziehen sich auf ein Phänomen, das eine eingetretene oder drohende Schädigung der Umwelt beschreibt. Dabei können sich die Indikatoren nicht nur auf die naturale Umwelt beziehen, sondern auch auf die vom Menschen geschaffene kulturale Umwelt (z. B. Gebäude) und den Menschen selbst.

In Textabschnitten, die sich allgemein auf Schäden beziehen, werden die Indikatorwörter nicht gekennzeichnet. Die Textstellen müssen sich immer auf ein konkretes Ereignis beziehen (bei Texten, die mehrere Ereignisse innerhalb eines bestimmten Zeitraums analysieren, muss sich der Indikator auf eines oder mehrere dieser beziehen). Dies ist notwendig, um das Schadensereignis zeitlich und räumlich zuordnen zu können; die Einbeziehung allgemeiner Textabschnitte würde die Auswertung verfälschen. Manche Texte wechseln zwischen allgemeinen und spezifischen Abschnitten oder behandeln nach einem

\footnotetext{
${ }^{253}$ Wobei der Begriff stets im Sinne von Umweltschaden verwendet werden muss.

254 Dies kann z. B. vorkommen, wenn bei der Umwandlung der Dateien von PDF-Format in ein Word-Format ein Wort mit Bindestrich getrennt ist oder ein Buchstabe falsch interpretiert wird (z. B. e statt a).
} 
allgemeinen Teil ein bestimmtes Beispiel. In diesen Texten werden also in manchen Abschnitten keine Markierungen vorgenommen. Wenn in einem Absatz derselbe Punkt wiederholt ausgeführt wird (typischerweise erste Erwähnung im Einleitungssatz gefolgt von detaillierten Erläuterungen), wird nur einmal markiert, da sonst diese Textstelle überproportional bewertet würde.

\subsubsection{Ableitung der zugrundeliegenden Werte}

Nachdem der Indikator im Text gefunden wurde, werden dem Text zunächst einige inhaltliche Fakten entnommen. Das im Text behandelte Ereignis bzw. Phänomen sowie dessen Folgen (der Umweltschaden) werden benannt und bezüglich der geographischen und zeitlichen Tragweite eingeordnet. Dann wird das betroffene Schutzgut identifiziert. Anschließend werden aus dem Kontext die Gründe für die Unerwünschtheit des beschriebenen Umweltschadens abgeleitet. Zum einen liefert der Autor durch die Aspekte, die hinsichtlich des Umweltschadens beschrieben werden bzw. eben nicht beschrieben werden einen Hinweis darauf, welche Werte im Vordergrund stehen. Wird z. B. die Anzahl der verletzten Menschen genannt, nicht aber, ob auch Tiere betroffen waren, lässt diese Auslassung den Rückschluss zu, dass der Text vorwiegend unter dem anthropozentrischen Blickwinkel verfasst wurde. Zum anderen lässt die Art wie der Umweltschaden beschrieben wird, d. h. welche Worte benutzt werden, Rückschlüsse auf die zugrundeliegenden Werthaltungen zu. Stollberg-Rilinger führt aus, dass Werte durch symbolisches Handeln reproduziert werden und sich so $u$. a. in sprachlichen Formeln wiederfinden. ${ }^{255}$ Wird z. B. bei einem Text über Windkraftanlagen von einer „Verspargelung“ der Landschaft geschrieben, deutet dies auf eine Wertschätzung der Schönheit der unverbauten Landschaft hin. Diese Interpretation ist der entscheidende Schritt zur Offenlegung der implizit im Text vorhandenen Wertvorstellungen.

Um eine möglichst konstante und nachvollziehbare Analyse zu gewährleisten, werden zu jeder Wertekategorie die verschiedenen Ausprägungen mit den zugehörigen Codierungen dokumentiert. Die computergestützte Textanalyse ermöglicht die nachvollziehbare Dokumentation sämtlicher Fundstellen.

Über die aufzufindenden Wertvorstellungen können vorab nur Vermutungen angestellt werden. Es ist aber vollkommen ungewiss, welche Wertvorstellungen bzw. Wertekategorien dann tatsächlich aus den Texten herausgearbeitet werden. Insofern scheint es wenig hilfreich zu sein, vor Beginn der Auswertung Wertekategorien zu definieren. Dies entspricht

\footnotetext{
255 Vgl. Barbara Stollberg-Rilinger, Einleitung, in: Barbara Stollberg-Rilinger/Thomas Weller (Hrsg.), Wertekonflikte - Deutungskonflikte. Internationales Kolloquium des Sonderforschungsbereichs 496 an der Westfälischen Wilhelms-Universität Münster 19. - 20. Mai 2005, Münster 2007, 1. Aufl., S. 9-20, hier S. 10.
} 
auch dem Prinzip der Offenheit bei interpretativen Analysen, einem „rekonstruktiven Vorgehen, das einer Entdeckungslogik folgt. “256 Zudem ist das Ziel dieser Arbeit (siehe Abschnitt 1.2) eine deskriptive Aussage zu den Wertvorstellungen, nicht die Überprüfung einer vorab zu bildenden Hypothese. Die Wertekategorien werden daher nicht vorab festgelegt, sondern induktiv gebildet.

Sobald bei der Textinterpretation der erste Wert erkennbar ist, wird für diesen eine passende Kategorie definiert. Findet sich im weiteren Analyseverlauf derselbe oder ein ähnlicher Wert, wird dieser unter die bereits vorhandene Kategorie subsumiert. Passt der neue Wert nicht in die bereits vorhandene Kategorie, wird eine neue Kategorie gebildet und der Wert dieser zugeordnet. Die Wertekategorien werden also induktiv, aus dem Material heraus, gebildet. Nach diesem Verfahren werden 10 bis $50 \%$ der zu analysierenden Texte durchgearbeitet, bis kaum noch neue Kategorien definiert werden müssen. Im Anschluss wird das Kategoriensystem hinsichtlich Logik und passendem Abstraktionsgrad geprüft und gegebenenfalls überarbeitet. Falls Änderungen notwendig sind, wird das Material erneut durchgearbeitet. Nach Beendigung dieser qualitativen Analyse können die so gewonnenen Daten auch quantitativ ausgewertet werden. ${ }^{257}$

In Anlehnung an Merten ${ }^{258}$ sind beim Aufbau des Kategoriensystems die folgenden Qualitätsanforderungen zu beachten:

- Das Kategoriensystem soll theoretisch abgeleitet sein, es soll mit den Zielen der Untersuchung korrespondieren.

- Das Kategorienschema soll vollständig sein, es soll die Erfassung aller nur möglichen Inhalte gestatten.

- Die Kategorien sollen wechselseitig exklusiv angelegt sein.

- Die Kategorien sollen voneinander unabhängig sein.

- Die Kategorien sollen einem einheitlichen Klassifikationsprinzip genügen.

- Die Kategorien sollen eindeutig definiert sein.

Um der Forderung nach Vollständigkeit des Kategoriensystems zu entsprechen, wird in dem hier verwendeten offenen Kategoriensystem eine inhaltlich nicht definierte Residualklasse (,Sonstige“ $)^{259}$ eingefügt.

\footnotetext{
${ }^{256}$ Rosenthal, Rosenthal 2005, S. 55.

${ }^{257}$ Vgl. Philipp Mayring, Einführung in die qualitative Sozialforschung: Eine Anleitung zu qualitativem Denken, Weinheim, 2002, S. 116-117.

${ }^{258}$ Vgl. Klaus Merten, Inhaltsanalyse: Einführung in Theorie, Methode und Praxis, Opladen, 1995, S. 98-99.

${ }^{259}$ Vgl. ebenda, S. 99.
} 


\subsubsection{Stichprobenumfang und -ziehung}

Die Definition der Grundgesamtheit ist bei qualitativen Studien grundsätzlich ein Problem. Der zentrale Punkt ist, dass der Fall facettenreich erfasst wird. ${ }^{260}$ Für die vorliegende Fragestellung besteht die theoretische Grundgesamtheit aus allen wissenschaftlichen Texten zu Themen der Umweltgeschichte. Dass diese interdisziplinär geprägt ist, verstärkt die definitorische Grundschwierigkeit noch. „So sind umweltgeschichtliche Beiträge oft heterogen sowohl im Hinblick auf die Themen als auch hinsichtlich des konzeptuellen Rahmens. Dies spiegelt sich auch in einer nahezu unüberschaubaren Fülle lokaler und regionaler Zeitschriften wider, die umwelthistorisch relevante Beiträge enthalten." 261 Der genaue Umfang ist damit als unbekannt anzusehen. Des Weiteren sind die Merkmale der Grundgesamtheit kaum bestimmt: Es muss eine wissenschaftliche Abhandlung sein die in schriftlicher Form vorliegt, in deutscher oder englischer Sprache verfasst ist, sich mit einem Thema der Umweltgeschichte befasst und das Wort Umweltschaden oder ein Synonym (siehe Abschnitt 3.2.1) enthält. Aufgrund der Unbekannten hinsichtlich der Grundgesamtheit ist die Stichprobengröße vorab nicht definiert. Die Anzahl der zu analysierenden Texte ergibt sich aus der induktiven Kategorienbildung, die eine gewisse Anzahl an Texten erfordert, bis die Kategorien möglichst vollständig erfasst sind. Methodisch bedeutet das für die Ziehung der Stichprobe eine Kombination der von Merkens beschriebenen beiden Möglichkeiten: „Einerseits kann die Stichprobe vor dem Beginn der Untersuchung bezüglich bestimmter Merkmale festgelegt sein, das heißt, jedes Element der Stichprobe wird auf der Basis eines Kriterienrasters gezogen. Andererseits kann man die Stichproben auf der Basis des jeweils erreichten Erkenntnisstandes erweitern und ergänzen (theoretisches Sampling)." ${ }^{262}$ Vor Beginn der Untersuchung werden einige Auswahlkriterien definiert, die eine sinnvolle Eingrenzung der Stichprobengröße erlauben und zugleich eine möglichst gleichmäßig verteilte Stichprobe gewährleisten sollen. Insoweit erfolgt die Stichprobenziehung durch selektives Sampling. Insbesondere ist so die Klumpenbildung der Stichprobe zu vermeiden, damit z. B. nicht ein Lehrstuhl die Mehrheit der Autoren stellt oder ein besonders häufig analysiertes Phänomen die Stichprobe dominiert. Im weiteren Verlauf der Analyse wird dann das theoretische Sampling angewandt, d. h. der jeweilige Auswertungsstand bestimmt den Fortgang der Untersuchung. Der Umfang der Stichprobe lässt sich demnach erst am Ende der Untersuchung bestimmen. Wann die Stichprobengröße einen ausreichenden Umfang erreicht und die Analyse beendet werden kann, wird durch den Forschungsprozess

\footnotetext{
${ }^{260}$ Vgl. Hans Merkens, Auswahlverfahren, Sampling, Fallkonstruktion, in: Uwe Flick/Ernst von Kardorff/Ines Steinke (Hrsg.), Qualitative Forschung. Ein Handbuch, Reinbek bei Hamburg 2009, S. 286-298, hier S. 291.

${ }^{261}$ Kreye et al., Kreye et al. 2009, S. 6.

${ }^{262}$ Merkens, S. 291-292.
} 
bestimmt. Oder, wie Rosenthal es formuliert „wenn wir im theoretischen Sinne nichts Neues mehr entdecken.“263

Auch im Zusammenhang mit dem Verfahren der induktiven Kategorienbildung ist die Stichprobengröße von Belang. Nach Mayring sollten nach dem Durcharbeiten von 10 bis $50 \%$ des Materials kaum noch neue Kategorien gebildet werden müssen. ${ }^{264}$ Das hat Auswirkungen auf die Reihenfolge der Stichprobenziehung, zumindest aber auf die Reihenfolge der Bearbeitung. Die ersten 10 bis $50 \%$ des Materials bzw. so viele Texte bis keine neuen Kategorien mehr erforderlich sind, müssen (innerhalb der Auswahlkriterien) eine möglichst heterogene Menge bilden, um möglichst alle erforderlichen Kategorien herausfiltern zu können. Im Umkehrschluss gibt das Verfahren der induktiven Kategorienbildung einen Hinweis auf die zu erwartende Größe der gesamten Stichprobe.

Hinsichtlich der Validität der Untersuchung ist anzumerken, dass nicht die Stichprobengröße das wichtigste Kriterium darstellt, also eine große Stichprobe nicht zwangsläufig zu einer höheren Validität führt. Vielmehr steht auch hier die Vielfältigkeit der einzelnen Fälle im Vordergrund.

Für die Stichprobenziehung wurde zunächst eine Internetsuche nach deutschen Hochschulen mit einem Fachbereich Geschichte durchgeführt. Die Internetauftritte der 49 gefundenen Hochschulen ${ }^{265}$ wurden dann nach umweltgeschichtlichen Forschungsinhalten bzw. Forschenden durchsucht. ${ }^{266}$ An 19 der Universitäten wurden umweltgeschichtliche Lehrstühle oder entsprechende Forschungsschwerpunkte gefunden, an 5 dieser Hochschulen gab es einen eigenen Bereich ${ }^{267}$ zum Thema Umweltgeschichte. Schließlich wurden die dort veröffentlichten Publikationslisten nach potenziellen Texten durchsucht. Des Weiteren wurde in Bibliothekskatalogen nach potenziellen Texten in Sammelwerken gesucht. Anschließend wurden die online verfügbaren Archive der folgenden Zeitschriftenjahrgänge nach geeigneten Beiträgen durchsucht: Environmental History 2000-2014, GAIA 2009-2014, Environment and History 1995-2014, Studienarchiv Umweltgeschichte 1995-2014, Journal of World History 1996-2014, Journal of Interdisciplinary History 1998-2014. Weiterhin wurden die Zeitschriften Natur und Recht, Environment and Behavior, Environmental Values, Historische Zeitschrift, Historische Anthropologie, Zeitschrift für Umweltpolitik \& Umweltrecht,

\footnotetext{
263 Rosenthal, Rosenthal 2005, S. 87

${ }^{264}$ Vgl. Mayring, Mayring 2002, S. 117

265 Ergebnis der Suche nach „Geschichte“ auf der Internetseite von www.studieren.de am 15.07.2014. Das Suchergebnis ist in Form einer Tabelle in Anhang A abgebildet.

${ }^{266}$ Sowohl über die Suchfunktion der jeweiligen Hochschulinternetseite mit dem Suchwort „Umweltgeschichte“ als auch über eine manuelle Suche auf den Seiten des Fachbereichs Geschichte.

${ }^{267}$ Lehrstuhl/ Arbeitsstelle/ Fachbereich/ Abteilung
} 
Historische Sozialforschung, History and Theory zunächst in Betracht gezogen, deren Inhalte erwiesen sich jedoch nach stichprobenartiger Sichtung als nicht geeignet.

Als Such- und Auswahlkriterien für die Texte dienen zum einen der Themenbereich Umweltgeschichte und zum anderen der Titel und ggfs. das Inhaltsverzeichnis der jeweiligen Publikation. Titel bzw. Inhaltsverzeichnis sollten vermuten lassen, dass ein Umweltschaden behandelt wird (siehe auch Abschnitt 3.2.4). Die so herausgefilterten Texte werden anschließend gesichtet, d. h. „quergelesen“, ob tatsächlich ein Umweltschaden beschrieben wird und evtl. auch schon ein Indikator auftaucht. Nach dieser weiteren Auswahlstufe werden die Texte in ein einheitliches elektronisches Format (MS Word) konvertiert ${ }^{268}$ und in MAXQDA importiert. Anschließend wird die lexikalische Suche durchgeführt. Dann werden alle Fundstellen im jeweiligen Kontext geprüft, ob tatsächlich ein Indikator gefunden wurde. Falls dem so ist, wird die Fundstelle farbig markiert, codiert und der Text dann analysiert, d.h. Wertefundstellen werden markiert (codiert) und einem Wert zugeordnet. Alle analysierten Texte sind im Anhang B aufgelistet.

\subsubsection{Auswahlkriterien der umweltgeschichtlichen Texte}

Es muss sich um einen wissenschaftlichen Text handeln, der schriftlich (ausformuliert) vorliegt und in Deutsch oder Englisch verfasst ist. Damit sind z. B. Zeitungsartikel oder Vorträge ausgeschlossen. Der Umfang der Texte sollte vergleichbar sein. Der Fokus liegt demnach auf Aufsätzen in Sammelwerken und Zeitschriftenartikeln.

Des Weiteren muss der Text veröffentlicht sein, $d . h$. der Text muss über Bibliotheken oder online verfügbar sein.

Der Text muss den Indikator „Umweltschaden“ oder ein Synonym enthalten (siehe Aufzählungen unter 3.2.1).

Hinsichtlich des Zeitpunktes der Veröffentlichung werden zunächst keine Einschränkungen gemacht. Da die Umweltgeschichte als wissenschaftliche Disziplin noch relativ jung ist, ist auch der fragliche Zeitraum eher kurz. Gegebenenfalls kann der Zeitraum nach dem selektiven Sampling eingegrenzt werden, um das theoretische Sampling effizienter zu gestalten. Gleiches gilt für den Zeitpunkt bzw. -raum des dem Text zugrunde liegenden Phänomens und des daraus resultierenden Schadens. Hier dürfte der zeitliche Horizont allerdings um einiges größer sein, da die Themen der Umweltgeschichte keine zeitliche Beschränkung kennen.

\footnotetext{
${ }^{268}$ Die Konvertierung erfolgt unter Verwendung der Software Omnipage.
} 
Spätestens während des zweiten Materialdurchgangs (theoretisches Sampling) ist zur Gewährleistung der qualitativen Repräsentativität auf eine möglichst gleichmäßige Verteilung der Themen zu achten. So sollten ephemere bis langfristige Phänomene genauso vertreten sein wie regionale bis globale Schadensausprägungen. Dafür wird die Schadensdauer und /ausdehnung (Fläche) des im Text behandelten Umweltschadens erfasst, gleiches gilt für das zugrundeliegende Ereignis, das den Schaden ausgelöst hat. So lassen sich zum einen Ereignis und Schaden in Relation setzen und zum anderen sicherstellen, dass die Untersuchung möglichst verschiedene Ausprägungen von Ereignissen bzw. Schäden erfasst. Weiterhin sollten die Texte in verschiedenen Jahren publiziert worden sein.

\subsubsection{Metadaten zu den Texten}

$\mathrm{Zu}$ jedem Text werden einige Metadaten aufgenommen. Neben dem Jahr der Veröffentlichung, der verwendeten Sprache und der übergeordneten Publikation in welcher der Beitrag erschienen ist, werden der Name des Verfassers und die Hochschule, der der Verfasser angehört, erfasst. Bei Beiträgen in Sammelwerken oder Zeitschriften wird der Text derjenigen Universität zugeordnet, an welcher der Autor des Beitrags zum Zeitpunkt der Veröffentlichung tätig war. Gibt es keine Hochschulzugehörigkeit oder ist diese unklar, wird der Text derjenigen Universität zugeordnet an welcher der Herausgeber zum Zeitpunkt der Veröffentlichung tätig war; ggfs. wird keine Hochschule zugeordnet (n.a.). Gleiches gilt für die Zuordnung des Herkunftslandes. Ist die Hochschulzugehörigkeit zum Zeitpunkt der Veröffentlichung nicht ohne Weiteres ersichtlich, wird der Text derjenigen Universität zugeordnet, an welcher der Autor bzw. der Herausgeber zum Zeitpunkt der Textanalyse tätig ist. Bei mehreren Autoren respektive verschiedenen Hochschulzugehörigkeiten wird der Text dem erstgenannten Autor zugeordnet. Das zugeordnete Land ergibt sich aus dem Standort der Hochschule. 


\section{Auswertung der Daten}

\subsection{Systematik der Auswertung}

Die Auswertung der Daten erfolgt in sechs Schritten. Zuerst werden in Abschnitt 4.2 die Indikatoren sowie der Aufbau und die Entstehung des Wertekategoriensystems dargestellt. Anschließend werden die enthaltenen Werte bzw. Wertekategorien im Einzelnen beschrieben.

Anhand der erhobenen Metadaten folgen in Abschnitt 4.3 Auswertungen und Beschreibungen zur Zusammensetzung der Stichprobe.

Danach zeigt Abschnitt 4.4 Auswertungen zur Anzahl der Indikator- und Wertecodierungen sowie Vergleiche und Zusammenhänge zwischen Indikatoren, Wertecodierungen und anderen Parametern wie z. B. dem Jahr der Veröffentlichung.

Abschnitt 4.5 zeigt zunächst eine Übersicht der Stichprobe mit den behandelten Themen bzw. Schäden, bevor die Daten zu Ereignisfläche und -dauer sowie zu Schadensfläche und dauer der untersuchten Texte ausgewertet werden. ${ }^{269}$

Im Abschnitt 4.6 erfolgt eine Betrachtung nach Wertekategoriegruppen. Zunächst werden die drei Paare anthropozentrisch/nicht-anthropozentrisch, materiell/immateriell und Existenzialwerte/Nicht-Existenzialwerte untersucht und auch nach Ländern verglichen. Aufgrund ihrer besonderen Stellung als übergeschichtliche Werte ${ }^{270}$ wird dann die Gruppe der Existenzialwerte näher betrachtet, bevor eine Auswertung nach den beiden Kategorien „nutzungsabhängige Werte“ und „nutzungsunabhängige Werte“ erfolgt.

\subsection{Vorstellung Indikatoren und Wertekategoriensystem}

Das verwendete Kategoriensystem gliedert sich in zwei Bereiche. Im ersten Bereich befinden sich die Indikatoren. Abbildung 5 zeigt eine Übersicht mit den fünf enthaltenen Indikatoren. Jedem Indikator wurden verschiedene Begriffe zugeordnet, nach denen im Text gesucht wurde (siehe hierzu auch Abschnitt 3.2.1). Die in Klammern angegebenen Abkürzungen dienten der Übersichtlichkeit bei der Arbeit mit der Analysesoftware und sind der Vollständigkeit halber mit angegeben.

\footnotetext{
${ }^{269}$ Die Ereignisfläche ist die Fläche, die das dem Schaden zugrundeliegende Ereignis beansprucht (z. B. bei einem Erdbeben die Fläche der Erschütterungen). Die Ereignisdauer ist die Zeitspanne, die das dem Schaden zugrundeliegende Ereignis angedauert hat (z. B. die Dauer eines Erdbebens). Die Schadensfläche ist die Fläche, die Schädigungen im Zusammenhang mit dem zugrundeliegenden Ereignis aufweist. Die Schadensdauer ist die Zeitspanne, die der Schaden anhält (sei es bis er beseitigt wurde oder bis sich die Natur regeneriert hat).

${ }^{270}$ Vgl. Fußnote 170 , S. 37
} 


\author{
Katastrophe (KA) \\ Schaden u. Ä. (SC) \\ Umweltbedrohung (UB) \\ Umweltschaden (US) \\ Umweltzerstörung (UZ)
}

\title{
Abbildung 5: Indikatoren
}

Der zweite Bereich beinhaltet die Wertekategorien und teilt sich in anthropozentrische sowie nicht anthropozentrische Wertekategorien (gekennzeichnet durch das vorangestellte „ANT“ für anthropozentrisch). Die Unterscheidung wurde in Abschnitt 2.2.5 diskutiert. In der vorliegenden Untersuchung sind Wertekategorien dann als anthropozentrisch bezeichnet, wenn der Mensch als Bezugspunkt erforderlich ist. Ist die Wertekategorie auch ohne Menschen ${ }^{271}$ denkbar, wird sie als nicht-anthropozentrisch eingeordnet. Abbildung 6 zeigt eine Übersicht der Wertekategorien.

Während der Phase der induktiven Kategorienbildung entstanden die Kategorien wie in der linken Spalte abgebildet. In dieser Arbeitsphase gab es auch eine Residualklasse „Sonstige“, deren Inhalt zum Abschluss der Kategorienbildung entweder einer vorhandenen Kategorie zugeordnet wurde oder eine neue Kategorie bildete (vgl. Abschnitt 3.2.2). Nach 18 Texten wurde die Kategorienbildung beendet. ${ }^{272}$ Aufgrund der Anzahl der Fundstellen wurden die Kategorien Gesundheit (GE) und Lebensgrundlage (LG) weiter untergliedert, um eine differenzierte Auswertung zu ermöglichen. Dieser Arbeitsstand ist in der mittleren Spalte dargestellt. Nach der Bearbeitung aller Texte wurde ebenfalls aufgrund der Anzahl der Fundstellen auch die Kategorie Nahrung (NA) weiter untergliedert. Die rechten Spalte zeigt somit die Liste der Wertekategorien am Ende der Textanalyse, die gleichzeitig die Basis der folgenden Auswertungen bildet.

Im Folgenden werden die einzelnen Wertekategorien erläutert und es wird definiert, worin der Schaden besteht, der der Wertekategorie zugrunde liegt. Zudem werden die Kategorien gegeneinander abgegrenzt, dort wo dies nötig scheint. Grundsätzlich sind Doppelzuordnungen von Fundstellen möglich, wenn der Sachverhalt zwei Wertekategorien an-

\footnotetext{
${ }^{271}$ Wie schon in Abschnitt 2.2.5 dargelegt, liegt es in der Natur der Sache, dass die Zuordnung von Werten immer durch den Menschen erfolgt. Da dies jedoch zu ausschließlich anthropozentrischen Werten führt, wurde die o. g. Aufteilung gewählt.

272 Diese 18 Texte entsprechen 10 bis 50\% der Stichprobengröße (vgl. Mayring, Mayring 2002, S. 117). Insgesamt wurden 54 Texte analysiert. Die Stichprobengröße weist damit einen angemessenen Umfang auf.
} 
spricht. ${ }^{273}$ Bei der Untergliederung der Wertekategorie „Gesundheit“ in „Hunger“, „Krankheit, Verletzungen“ und "Tod“ ist zu beachten, dass diese deshalb so benannt sind, damit sofort der Schaden zu erkennen ist, der diesem Wert zugrunde liegt und so eine einfache Verbindung zu den Fundstellen in den Texten herzustellen ist. Einen Wert „Hunger“ z. B. gibt es eigentlich nicht, sondern nur den entgegengesetzten Wert „satt sein“. Dies ist bei der weiteren Auswertung zu beachten.

\begin{tabular}{|c|c|c|c|}
\hline \multirow{6}{*}{$\begin{array}{l}\text { nicht } \\
\text { ANT }\end{array}$} & Artenbestände (AB) & Artenbestände (AB) & Artenbestände (AB) \\
\hline & Artenvielfalt (AV) & Artenvielfalt (AV) & Artenvielfalt (AV) \\
\hline & Nachhaltigkeit (NA) & Nachhaltigkeit (NA) & Nachhaltigkeit (NA) \\
\hline & Okosystem (OS) & Okosystem (OS) & Ökosystem (OS) \\
\hline & Tiere (TI) & Tiere $(\mathrm{TI})$ & Tiere (TI) \\
\hline & Unberührte Natur (UN) & Unberührte Natur (UN) & Unberührte Natur (UN) \\
\hline \multirow{21}{*}{ ANT } & $\begin{array}{l}\text { Erholung, Freizeit, Komfort } \\
\text { (EF) }\end{array}$ & $\begin{array}{l}\text { Erholung, Freizeit, Komfort } \\
\text { (EF) }\end{array}$ & $\begin{array}{l}\text { Erholung, Freizeit, Komfort } \\
\text { (EF) }\end{array}$ \\
\hline & Einkommensquelle (EQ) & Einkommensquelle (EQ) & Einkommensquelle (EQ) \\
\hline & Identität (ID) & Identität (ID) & Identität (ID) \\
\hline & Imageschaden (IM) & Imageschaden (IM) & Imageschaden (IM) \\
\hline & Infrastruktur (IS) & Infrastruktur (IS) & Infrastruktur (IS) \\
\hline & $\begin{array}{l}\text { Moralische Verantwortung, } \\
\text { Pflicht (MO) }\end{array}$ & $\begin{array}{l}\text { Moralische Verantwortung, } \\
\text { Pflicht (MO) }\end{array}$ & $\begin{array}{l}\text { Moralische Verantwortung, } \\
\text { Pflicht (MO) }\end{array}$ \\
\hline & Okonomie (OK) & Okonomie (OK) & Okonomie (OK) \\
\hline & Religion, Glaube (RE) & Religion, Glaube (RE) & Religion, Glaube (RE) \\
\hline & Rohstofflieferant (RL) & Rohstofflieferant (RL) & Rohstofflieferant (RL) \\
\hline & $\begin{array}{l}\text { Schönheit der } \\
\text { Natur (SCO) }\end{array}$ & $\begin{array}{l}\text { Schönheit der } \\
\text { Natur (SCÖ) }\end{array}$ & $\begin{array}{l}\text { Schönheit der } \\
\text { Natur (SCO) }\end{array}$ \\
\hline & Stabilität (polit.) (ST) & Stabilität (polit.) (ST) & Stabilität (polit.) (ST) \\
\hline & Tradition (TR) & Tradition (TR) & Tradition (TR) \\
\hline & Wissen, Erkenntnis (WI) & Wissen, Erkenntnis (WI) & Wissen, Erkenntnis (WI) \\
\hline & Zuhause, Eigentum (ZH) & Zuhause, Eigentum (ZH) & Zuhause, Eigentum (ZH) \\
\hline & & Hunger (HU) & Hunger (HU) \\
\hline & Gesundheit (GE) & $\begin{array}{l}\text { Krankheit, Verletzungen } \\
\text { (KR) }\end{array}$ & $\begin{array}{l}\text { Krankheit, Verletzungen } \\
\text { (KR) }\end{array}$ \\
\hline & & Tod (TO) & Tod (TO) \\
\hline & \multirow{4}{*}{ Lebensgrundlage (LG) } & Luft (LU) & Luft (LU) \\
\hline & & Wasser (WA) & Wasser (WA) \\
\hline & & & $\begin{array}{l}\text { Boden, Felder (BO) } \\
\text { Frnte Saataut (FR) }\end{array}$ \\
\hline & & Nahrung (NA) & \begin{tabular}{|l} 
Ernte, Saatgut (ER) \\
Nahrungsmittel (NM)
\end{tabular} \\
\hline
\end{tabular}

Abbildung 6: Wertekategorien (aus der Textanalyse dieser Arbeit)

Artenbestände $(A B)$ : Dezimieren von Beständen einer oder mehrerer Arten sowohl in Flora als auch Fauna. Dies kann direkt oder indirekt durch Vernichtung, Verkleinerung oder Verschlechterung des Lebensraumes geschehen.

Artenvielfalt (AV): Auslöschen von Beständen einer oder mehrerer Arten sowohl in Flora als auch Fauna.

\footnotetext{
${ }^{273}$ Z. B. im Falle von Kleinbauern, die gleichzeitig Selbstversorger sind, bei einer schlechten Ernte: hier wird zum einen die Einkommensquelle der Familie verringert, zugleich aber auch die Nahrungsgrundlage der Familie.
} 
Nachhaltigkeit (NA): Beeinträchtigung der zur Erhaltung notwendigen Regenerationsfähigkeit von Ressourcen. Meines Erachtens gehört diese Wertekategorie zu der Gruppe der nicht-anthropozentrischen Wertekategorien, denn, wie oben ausgeführt, ist diese Kategorie auch ohne Menschen denkbar. Anthropozentrische Werte beruhen jedoch auf der Beziehung zwischen Mensch und Natur. ${ }^{274}$ Die zur Erhaltung notwendige Regenerationsfähigkeit von Ressourcen ist hingegen unabhängig vom Menschen zu sehen. Vielmehr kann hier die Frage auftauchen, ob der Schaden im Bereich der natürlichen Schwankungsbreite liegt, da die Natur an sich kein statisches System ist, sondern ständig in Bewegung. Der Umstand, dass der Mensch manche Ressourcen nutzen möchte bzw. muss (z.B. Wasser) und daher auf deren nachhaltige Bewirtschaftung angewiesen ist, wird in den Kategorien „Lebensgrundlage“ berücksichtigt. Zudem wird die Art der Nutzung (z.B. Entspannung, Ernährung, Einkommen) durch den Menschen benötigt, um die Zuordnung zu der jeweiligen (anthropozentrischen) Wertekategorie zu bestimmen. Steht also die Nachhaltigkeit im Sinne einer allgemeinen Regenerationsfähigkeit im Vordergrund, wird die Fundstelle der Kategorie „Nachhaltigkeit“ zugeordnet.

Ökosystem (ÖS): Beeinträchtigung oder Zerstörung von Ökosystemen. In Abgrenzung zu anderen Kategorien wie z. B. AV (Artenvielfalt) wird hier in den Fundstellen das Stichwort Ökosystem genannt.

Tiere (TI): Beeinträchtigung der Gesundheit und Tod der Tiere ohne nähere Spezifizierung (sonst AB oder AV). Eingeschlossen sind Haus-, Nutz- und Wildtiere.

Unberührte Natur (UN): Natur bzw. Natur, die sich selbst überlassen wird als Wert an sich (insbesondere das amerikanische Konzept von „wilderness"), ohne Bewertung nach Schönheit oder Nutzen. Erhaltung z. B. als Nationalpark.

Erholung, Freizeit, Komfort (ANT/EF): Natur als Ort der Entspannung, als Erholungs/Urlaubsort. Komfort kann z. B. durch Geruchs- oder Lärmbelästigung beeinträchtigt werden (ohne zwangsläufig die Gesundheit zu beeinträchtigen). Abgrenzung zu ÖK (Ökonomie): Steht der Tourismus im Sinne einer Einkommensquelle im Vordergrund, wird die Fundstelle ÖK (Ökonomie) zugeordnet.

Einkommensquelle (ANT/EQ): Verringerung oder Verlust von Naturerzeugnissen, die für einzelne Produzenten oder Individuen eine Einkommensquelle darstellen. Sind mehrere Produzenten oder Regionen betroffen, wird der Sachverhalt bei ÖK (Ökonomie) eingeordnet.

\footnotetext{
${ }^{274}$ Vgl. Potthast, Potthast 2000, S. 119.
} 
Identität (ANT/ID): Schäden an identitätsstiftender Natur oder Umwelt. Menschen identifizieren sich manchmal mit einer bestimmten Umgebung oder einem Phänomen. Auch sogenannte Wahrzeichen wie z. B. der Eiffelturm sind hier eingeschlossen.

Imageschaden (ANT/IM): Imageschaden für den Verursacher oder Verantwortlichen des Schadens.

Infrastruktur (ANT/IS): Beeinträchtigung oder Zerstörung von Strom-, Gas-, Wasserversorgung, Verkehrswegen oder öffentlichen Gebäuden.

Moralische Verantwortung, Pflicht (ANT/MO) $)^{275}$ : Fundstellen dieser Kategorie sprechen direkt von moralischer Verantwortung, Pflicht oder Respekt gegenüber Flora, Fauna und Menschen.

Ökonomie (ANT/ÖK): Kosten aufgrund von Umweltschäden für Beseitigung, Lagerung, Produktionsausfälle, nicht nutzbare Restbestände (nach Produktverbot), allgemeine Beeinträchtigung des Tourismus, Preissteigerung/-verfall. Auch Fundstellen, in denen ein Umweltschaden monetär bewertet wird ohne näher zu erläutern, worin der Schaden bzw. Verlust besteht. Abgrenzung zu EQ (Einkommensquelle): Die Kosten betreffen nicht nur einzelne Produktionsstätten, sondern mehrere oder ganze Industrien.

Religion (ANT/RE): Beeinträchtigung der Umwelt als Objekt religiöser Handlungen, aber auch Verantwortung für die Umwelt, die aus religiösen Ansichten abgeleitet wird.

Rohstofflieferant (ANT/RL): Beeinträchtigung oder Zerstörung der Möglichkeit zur Rohstoffgewinnung.

Schönheit der Natur (ANT/SCÖ): Beeinträchtigungen des Landschaftsbildes, zumeist durch Eingriffe des Menschen wie z. B. Tagebau oder Anlage von Skipisten.

Stabilität, politisch (ANT/ST): z. B. Bürgerunruhen oder Aufstände in Folge des Umweltschadens.

Tradition (ANT/TR): Verlust von traditionellen Ritualen bzw. langjährigen Traditionen.

Wissen, Erkenntnis (ANT/WI) $)^{276}$ : Erkenntnisgewinn als Wert an sich.

\footnotetext{
275 Diese Kategorie unterscheidet sich von den anderen. Während die anderen Wertekategorien die Frage beantworten, warum ein Schaden unerwünscht ist (weil dadurch ein bestimmtes Schutzgut beeinträchtigt wird), ist diese Kategorie unabhängig vom betroffenen Schutzgut.

276 Siehe Fußnote 275. Darüberhinaus sind die Fundstellen dieser Kategorie z.T. sogar unabhängig vom Umweltschaden. Mit insgesamt nur drei Fundstellen wurde diese Kategorie der Vollständigkeit halber aufgenommen, hat aber eher marginale Bedeutung.
} 
Zuhause, Eigentum (ANT/ZH): Evakuierung, zerstörte Häuser und sonstiges, was zur temporären oder dauerhaften Nichtnutzbarkeit (oder Einschränkung der Nutzbarkeit) des Zuhauses oder zum Verlust von Hab und Gut führt. Weitere Stichwörter sind Heimat und Lebensraum.

Gesundheit (ANT/GE): Sämtliche physischen und psychischen Beeinträchtigungen von Menschen bis hin zum Tod. Dies schließt die Möglichkeit der Fortpflanzung mit ein.

Hunger (ANT/GE/HU): Minderversorgung des Menschen mit Nahrungsmitteln, in dessen Folge Hunger entsteht. Solange es im Text keinen Hinweis auf gesundheitliche Folgen des Hungers gibt, wird Hunger dieser Kategorie zugeordnet.

Krankheit, Verletzungen (ANT/GE/KR): Beeinträchtigung (auch das Risiko einer Beeinträchtigung) der menschlichen Gesundheit ohne Todesfolge.

Tod (ANT/GE/TO): Menschliche Todesopfer. Die Fundstelle wird hier eingeordnet, wenn explizit von Tod berichtet wird; potenziell tödliche Krankheiten werden bei GE/KR eingeordnet.

Lebensgrundlage (ANT/LG): Sowohl bei direkter Nichtverfügbarkeit (z. B. Bauern, Selbstversorger) als auch bei indirekter Nichtverfügbarkeit oder eingeschränkter Verfügbarkeit (z. B. Preissteigerungen, Unterbrechung des Transportweges, schlechte Luftqualität) von Luft, Wasser, Nahrung.

Luft (ANT/LG/LU): Beeinträchtigung der Luftqualität.

Wasser (ANT/LG/WA): Beeinträchtigung der Wasserqualität oder der Verfügbarkeit von Wasser.

Nahrung (ANT/LG/NA): Schäden an Ackerland oder Ernte, aber auch Verlust von Nutz- oder Arbeitstieren. Abgrenzung zu GE: Wird im Text über Schäden o. Ä. an Nahrung berichtet, wird der Sachverhalt NA zugeordnet; wird hingegen über eine Hungersnot, also die Folge von Nahrungsmangel, berichtet, wird der Sachverhalt GE zugeordnet, da in diesem Fall die gesundheitliche Beeinträchtigung im Vordergrund steht.

Boden, Felder (ANT/LG/NA/BO): Schäden an Ackerboden wie Erosion, verschlechterte Fruchtbarkeit, Übernutzung, Verwüstung, Wüstenbildung. Auch Minderung oder Verlust von Arbeitskraft zum Bestellen der Felder (Mensch und Arbeitstiere).

Ernte, Saatgut (ANT/LG/NA/ER): Schlechte Ernte oder Ernteverlust sowie beschädigtes oder vernichtetes Saatgut. 
Nahrungsmittel (ANT/LG/NA/NM): Verfügbarkeit und Qualität von Nahrungsmitteln (Fleisch, Fisch, Getreide, ...). Eingeschränkte Verfügbarkeit zeigt sich auch durch Preissteigerungen. Abgrenzung zu ER: Nahrungsmittel meint explizit Nahrung für den Menschen, Ernte ist allgemeiner und kann sich z. B. auch auf Viehfutter beziehen.

\subsection{Auswertung der Metadaten}

Insgesamt wurden 54 Texte $^{277}$ analysiert. Davon sind 20 in deutscher Sprache und 34 in englischer Sprache verfasst. Die Aufsätze wurden zwischen 1988 und 2014 veröffentlicht. Abbildung 7 zeigt die Häufigkeitsverteilung. Daraus wird ersichtlich, dass nur $9 \%$ der analysierten Texte älter als 15 Jahre sind. 74\% der Aufsätze wurden 2007 oder später veröffentlicht. Der Fokus liegt somit auf Veröffentlichungen jüngeren Datums.

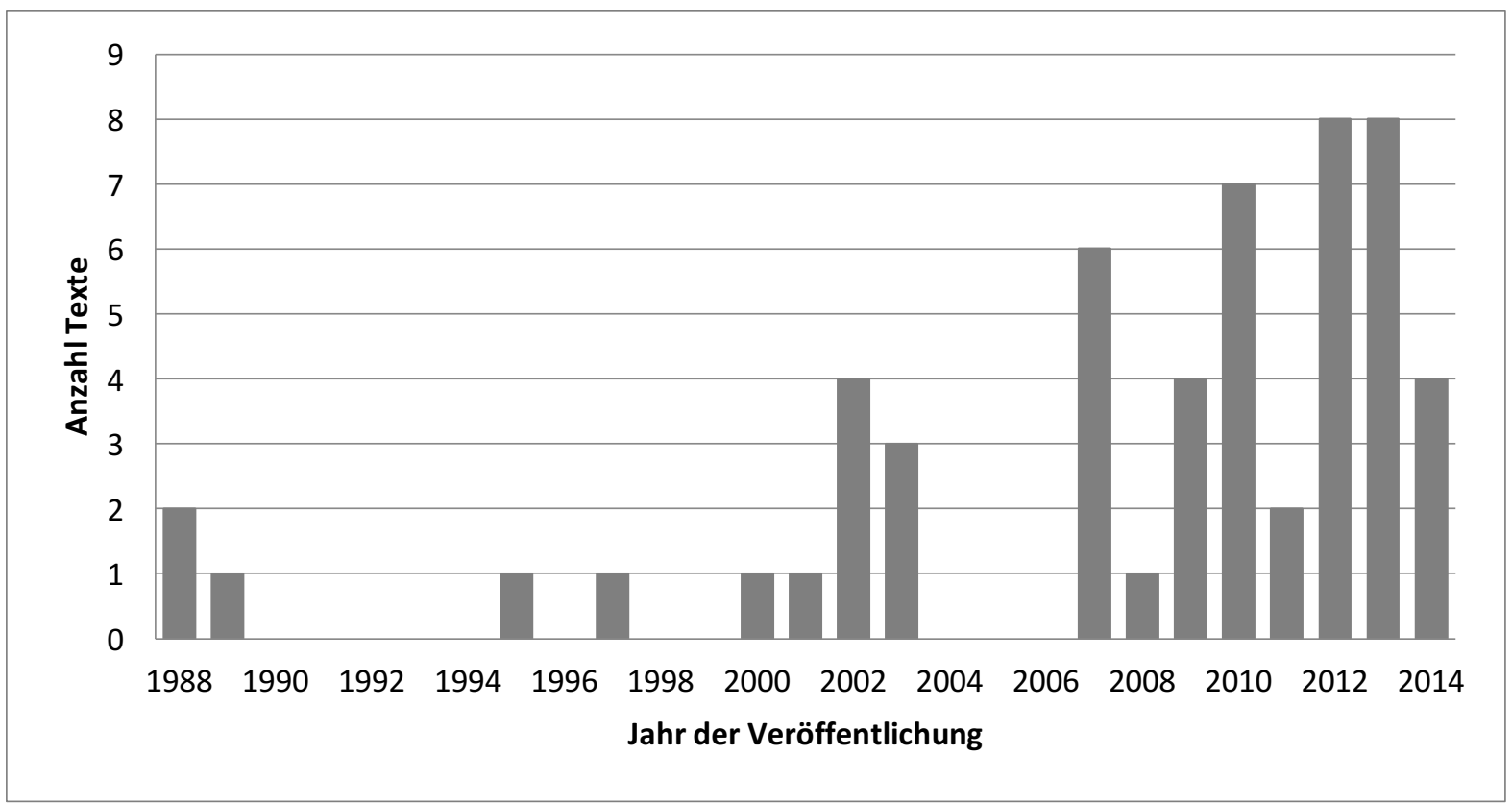

Abbildung 7: Jahr der Veröffentlichung mit jeweiliger Anzahl der Texte

${ }^{277}$ Der Umfang betrug in Summe 1.251 Seiten, d. h. im Durchschnitt war ein Text 23 Seiten lang. 


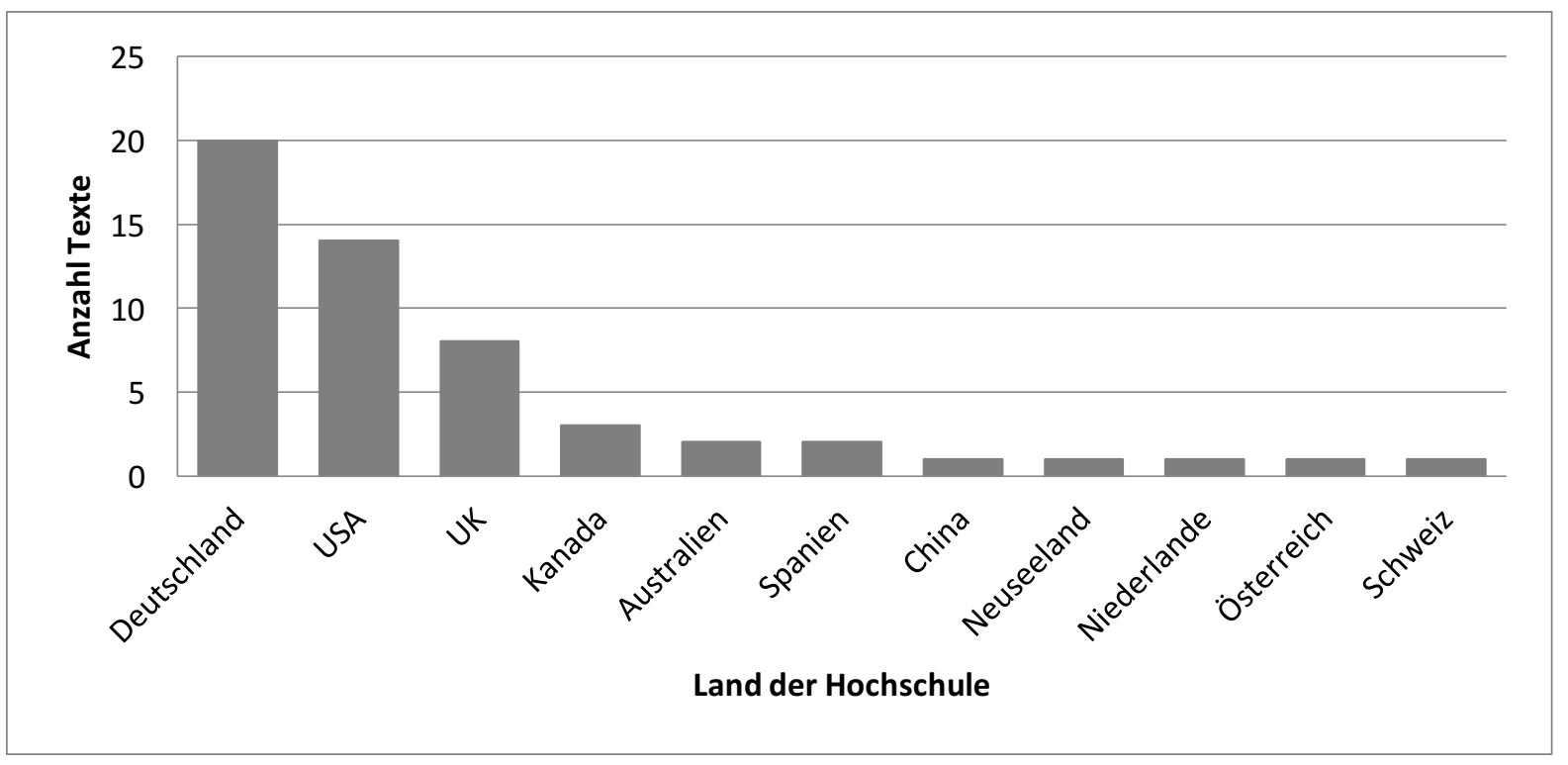

Abbildung 8: Land der Hochschule mit jeweiliger Anzahl der Texte

Bei der Hochschulzugehörigkeit der Verfasser gibt es eine breite Streuung, wobei deutsche Universitäten mit einem Anteil von 35\% am häufigsten vertreten sind. Insgesamt sind 41 verschiedene Hochschulen vertreten. Mehrfach vertreten sind Göttingen (5 Texte), Darmstadt (4 Texte), Neubrandenburg (3 Texte) und Hamburg (2 Texte). In Abbildung 8 ist dargestellt, in welchen Ländern die Hochschulen liegen bzw. die Anzahl der Texte aus dem jeweiligen Land. Hierbei ist zu beachten, dass die Hochschulzugehörigkeit nicht zwangsläufig mit der Sprache des Textes korreliert. So gibt es englischsprachige Texte von deutschen Hochschulen genauso wie deutsche Übersetzungen, die aus englischsprachigen Ländern stammen. Nach den Texten aus Deutschland kommen die meisten Beiträge aus den USA und dem Vereinigten Königreich. Diese drei Länder machen 78\% der Aufsätze aus.

Bei der fachlichen Zugehörigkeit der Autoren zu Natur- oder Geisteswissenschaften zeigt sich ein eindeutiges Bild: Von den 54 analysierten Texten stammen 45 (83\%) von Forschern mit geisteswissenschaftlichem Hintergrund, 40 davon aus dem Bereich Geschichtswissenschaften (74\% der Dokumente). 


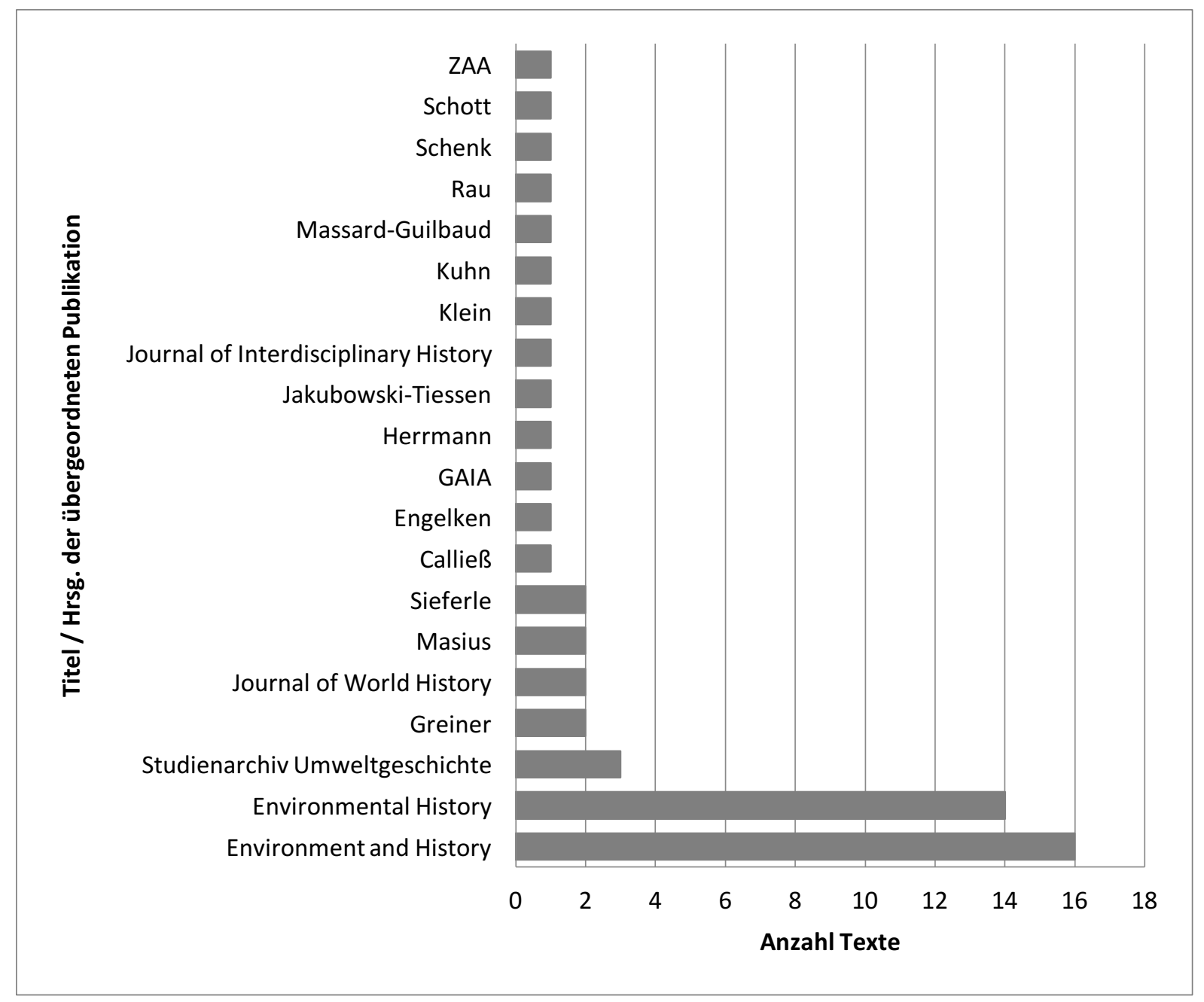

Abbildung 9: Übergeordnete Publikation des Textes

Die Abhandlungen wurden entweder in einem Sammelband oder in einer Fachzeitschrift veröffentlicht. $30 \%$ der Texte wurden Sammelbänden entnommen, $70 \%$ stammen aus Fachzeitschriften. Während den verwendeten Sammelwerken jeweils meist nur ein Beitrag entnommen wurde, wie Abbildung 9 zeigt, entstammen 16 der analysierten Texte (30\%) der Zeitschrift „Environment and History“ und weitere 14 (26\%) der Zeitschrift „Environmental History“. Die 16 Aufsätze aus „Environment and History“ wurden den Jahrgängen 1995-2013 entnommen, die 14 Aufsätze aus „Environmental History“ den Jahrgängen 2001-2014. ${ }^{278}$

\footnotetext{
${ }^{278}$ Aus der Anzahl der Jahrgänge, der durchschnittlichen Anzahl von Artikeln pro Ausgabe und der Anzahl der Ausgaben pro Jahr ergibt sich, dass von etwa 240 gesichteten Artikeln aus „Environmental History“ nur 14 ausgewählt wurden (6\%) und von etwa 380 Artikeln aus „Environment and History“ nur 16 ausgewählt wurden (4\%). Diese geringe Ausbeute scheint Sieferle zu widersprechen, der meint, dass sich die Umweltgeschichte mehrheitlich mit Umweltverschmutzung beschäftigt (vgl. Fußnote 105, S. 25).
} 


\subsection{Quantitative Auswertungen zu Indikator- und Wertecodierungen}

Es wurden insgesamt 874 Fundstellen mit Hinweisen auf Werte und 461 Fundstellen mit Indikatoren ${ }^{279}$ entdeckt. Abbildung 10 zeigt zunächst die Häufigkeitsverteilung der Indikatoren. Das Wort „Katastrophe“ kam demnach mit 147 Fundstellen in 28 Dokumenten am häufigsten vor. An zweiter Stelle folgt der Begriff „Umweltzerstörung“ mit 135 Fundstellen, wobei dieser Indikator in den meisten Dokumenten (37) gefunden wurde. Es folgen „Schaden“, „Umweltbedrohung“ und schließlich der Begriff „Umweltschaden“, der erst an letzter Stelle der Häufigkeitsverteilung mit 35 Fundstellen (in 16 Dokumenten) erscheint.

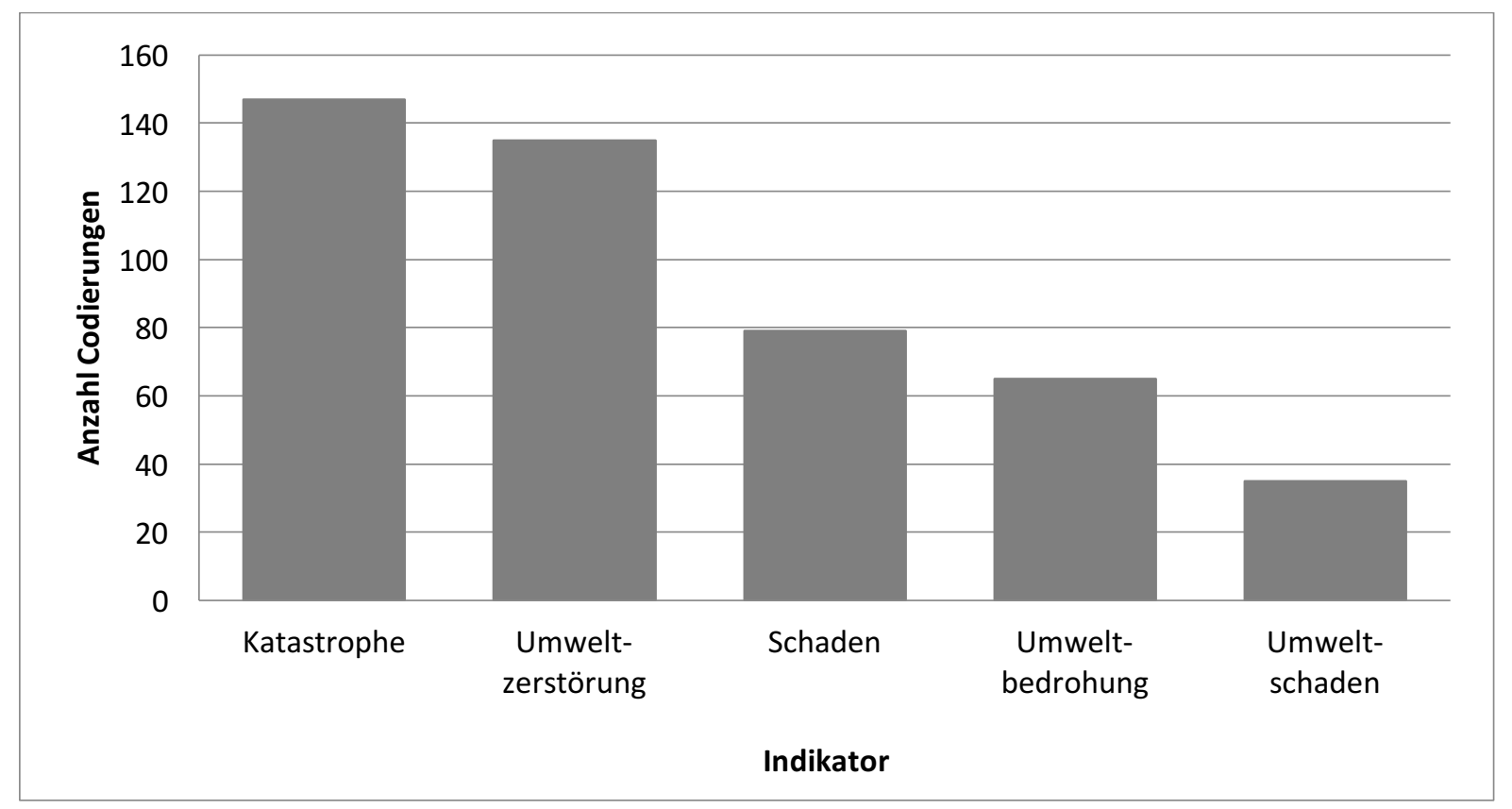

\section{Abbildung 10: Indikatoren (nach Anzahl Codierungen)}

Bei der Bearbeitung der Dokumente hat sich bestätigt, dass das Wort „Umweltschaden“ vergleichsweise selten explizit verwendet wird. Nichtsdestotrotz behandeln alle analysierten Texte Umweltschädigungen, wenngleich in unterschiedlicher Ausprägung (s.o.). Dieses Muster ist hinsichtlich der zugrunde liegenden Werte insofern interessant, als die Nichtverwendung des Begriffs „Umweltschaden“ darauf hindeuten könnte, dass der Verfasser wertende Begriffe vermeiden möchte. Allerdings weisen Texte, in denen der Indikator „Umweltschaden“ nicht vorkommt, im Durchschnitt 16,7 Wertecodierungen auf, während der Durchschnitt bei 16,2 Wertecodierungen pro Text liegt und zeigen damit nur eine sehr geringe Abweichung. In Abbildung 16 wird diese Fragestellung weiter vertieft.

Nun ist es so, dass pro Text nicht nur ein Indikator vorkommen kann, sondern mehrere. Abbildung 11 zeigt die Anzahl der Texte nach der im Text vorkommenden Anzahl von

\footnotetext{
${ }^{279}$ Zugunsten der Übersichtlichkeit ist hier jeweils nur der wichtigste Oberbegriff genannt. Eingeschlossen sind aber auch Synonyme sowie die englischen Begriffsäquivalente. Sämtliche Indikatoren werden in den Texten im Sinne von „Umweltschaden“ verwendet (vgl. Abschnitt 3.2.1).
} 
unterschiedlichen Indikatoren. Bei fast einem Viertel der Texte kommt demnach nur ein Indikator vor. Meistens jedoch (bei 33\%) sind es zwei Indikatoren. Die häufigsten Kombinationen sind hierbei „Katastrophe“ und „Umweltzerstörung" (11\%) sowie „Umweltzerstörung“ und „Umweltbedrohung“ (9\%). Bei weiteren 20\% der Texte sind drei Indikatoren enthalten und bei $20 \%$ sogar vier. Alle fünf Indikatoren kommen nur in $4 \%$ der Texte vor.

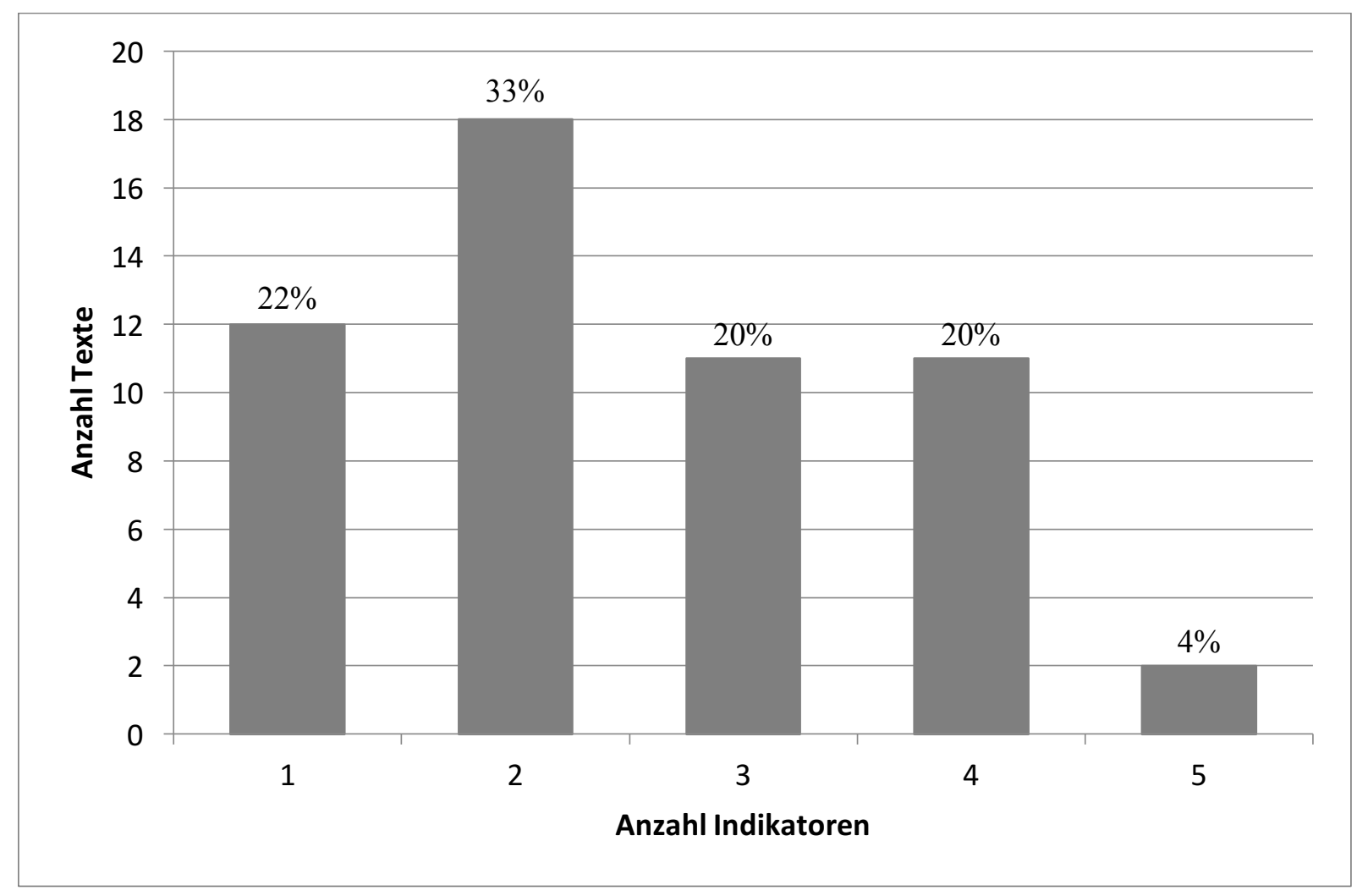

Abbildung 11: Anzahl Indikatoren (nach Anzahl Texte)

Ein Vergleich der Anzahl der pro Text verwendeten Indikatoren mit der durchschnittlichen Anzahl der Wertecodierungen pro Text zeigt keinen direkten Zusammenhang. Lediglich bei Dokumenten mit vier Indikatoren ergibt sich eine erhöhte Anzahl Wertecodierungen. Dasselbe gilt für den Vergleich mit der Anzahl gefundener Werte pro Text. ${ }^{280}$

Im Folgenden wird die Anzahl der Indikator- und Wertecodierungen in Relation gesetzt, verglichen und einige Zusammenhänge zwischen Indikatoren und Werten werden aufgezeigt. Abbildung 12 zeigt alle analysierten Texte als Punkte mit der Anzahl der Indikatorcodierungen und der Anzahl der Wertecodierungen als Koordinaten. Die eingezeichnete Trendlinie (über alle Punkte) deutet zwar einen Zusammenhang zwischen der Anzahl der Indikatorcodierungen und der Anzahl der Wertecodierungen an, aufgrund der großen Streuung ist der Zusammenhang allerdings sehr schwach ausgeprägt bzw. statistisch nicht signifikant. ${ }^{281}$ Auch bei der Betrachtung der Punkte nach Ländern lässt sich kein eindeutiger

280 Die Auswertung ergab folgende Zahlen: Anzahl Indikatoren (durchschnittliche Anzahl Wertecodierungen; durchschnittliche Anzahl Werte): 1(17;5), 2(16;7), 3(11;5), 4(21;9), 5(15;6). 
Trend feststellen, vielmehr ist die ganze Punktewolke eher heterogen verteilt. Tendenziell haben Texte aus dem UK mehr Wertecodierungen als Texte aus Deutschland.

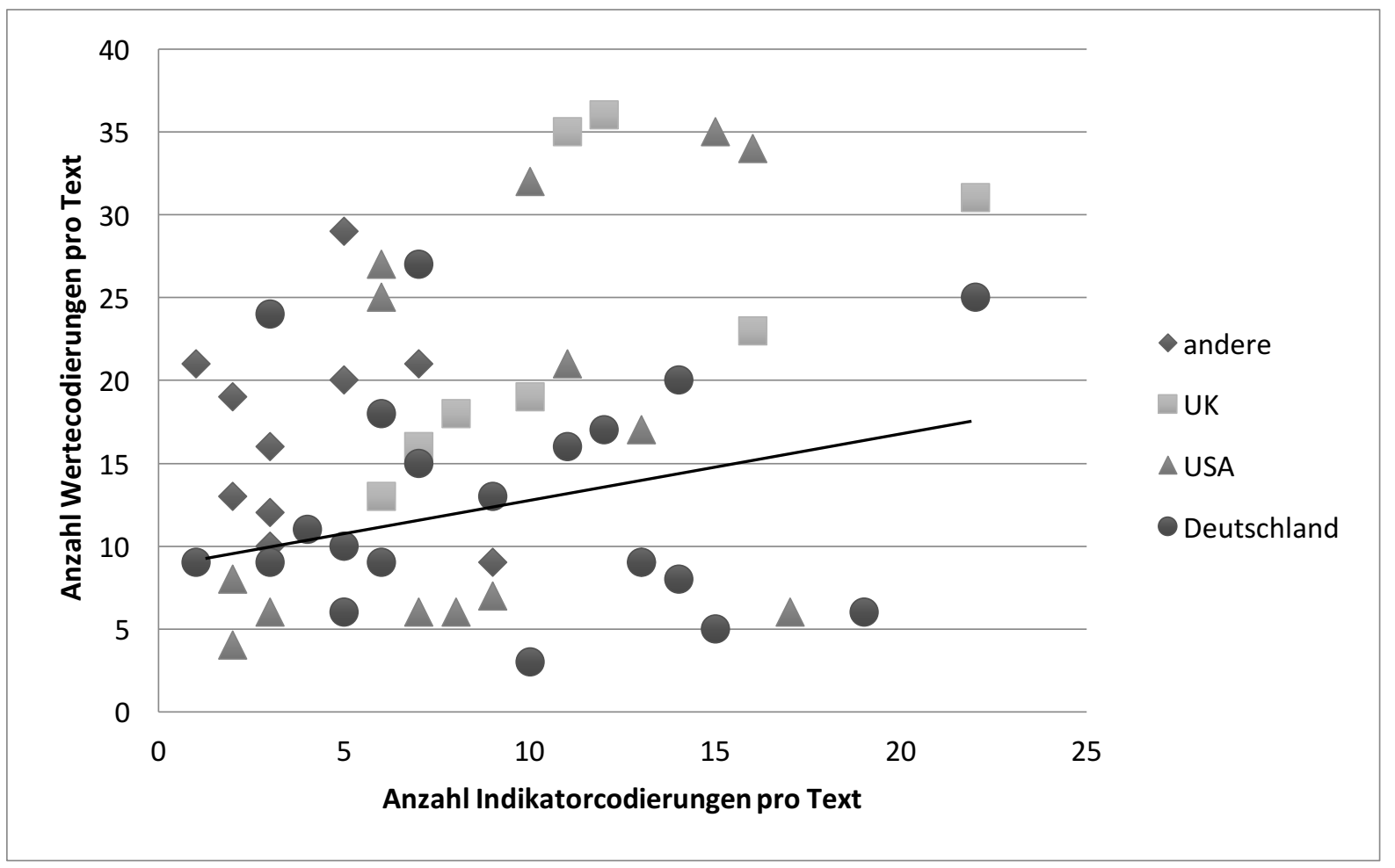

\section{Abbildung 12: Anzahl Codierungen pro Text (Indikator- zu Wertecodierungen)}

Als nächstes zeigt Abbildung 13 die durchschnittliche Anzahl der Werte- und Indikatorcodierungen nach dem Jahr der Publikation. Wenngleich es hier eine gewisse Schwankungsbreite gibt, bei Wertecodierungen stärker als bei Indikatorcodierungen, ist die Anzahl tendenziell stabil. Die beiden überdurchschnittlich hohen Anzahlen von Wertecodierungen in den Jahren 1995 und 2008 sind als Ausreißer zu werten. ${ }^{282}$ Die gestrichelte Linie zeigt den Verlauf ohne diese beiden Ausreißer. Insgesamt liegt die Anzahl der Wertecodierungen bis auf ein Jahr (2009) höher als die der Indikatorcodierungen. Das Verhältnis Werte- zu Indikatorcodierungen liegt im Durchschnitt aller Texte bei 3,0 und der Median bei 2,1.

\footnotetext{
${ }^{281}$ Das Bestimmtheitsmaß der beiden Variablen liegt mit $18 \%$ sehr niedrig $\left(R^{2}=0,18\right.$ bei einer angenommenen linearen Regression), d. h. es erklären sich nur $18 \%$ der Varianz aus dem Zusammenhang zwischen den beiden Variablen.

${ }^{282}$ Es handelt sich jeweils um nur einen Text (Themen: Korallensammeln am Great Barrier Reef sowie Abholzung und Agrarnot in Nordindien).
} 


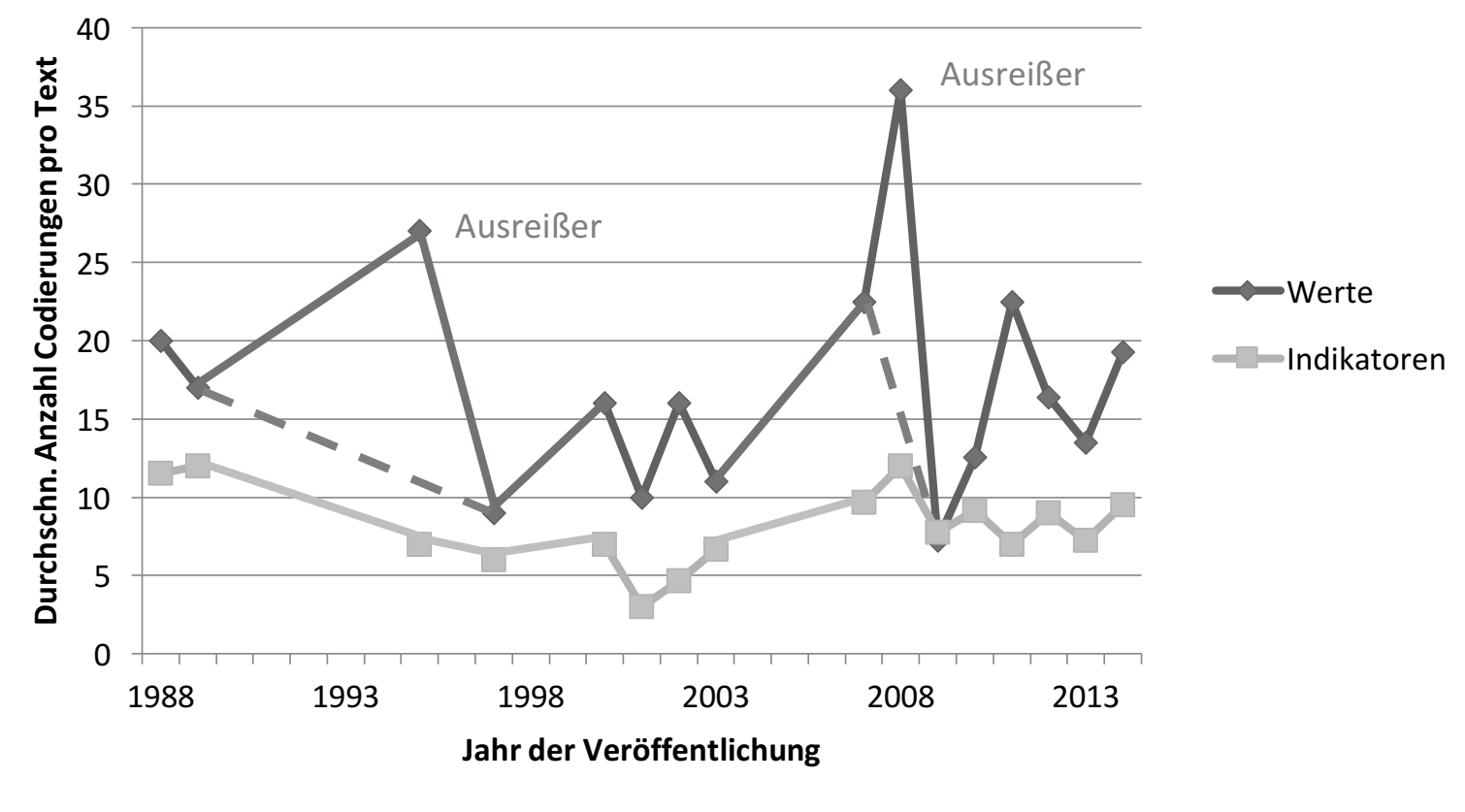

Abbildung 13: Anzahl Codierungen pro Text (nach Jahr der Veröffentlichung)

In Abbildung 14 ist die Anzahl der Codierungen pro Text nach Sprache bzw. Land dargestellt. Während der Durchschnitt aller Texte eine Anzahl von 16 Wertecodierungen pro Text ergibt, zeigen sich deutliche Abweichungen bei der Sortierung nach Sprache und Ländern. Deutsche Texte liegen mit durchschnittlich 12 Wertecodierungen 25\% unter dem Gesamtdurchschnitt. Im Gegensatz dazu haben englische Texte im Schnitt 17 Wertecodierungen - fast $50 \%$ mehr als deutsche Texte. Die Anzahl der Indikatorcodierungen weicht allerdings nicht in gleichem Maße ab. Hier unterscheiden sich deutsche und englische Texte fast gar nicht: während deutsche Texte durchschnittlich neun Indikatorcodierungen enthalten, sind es bei englischen Texten acht. Bei einer Sortierung nach den drei Ländern ${ }^{283}$ mit den meisten Beiträgen (Deutschland (D), die Vereinigten Staaten (USA) und das Vereinigte Königreich (UK)) gibt es bei den Werten für Texte aus Deutschland kaum Abweichungen, während es zwischen Texten aus den USA und dem UK deutliche Unterschiede gibt. Bei den Indikatorcodierungen liegen die Texte aus dem UK mit durchschnittlich 12 pro Text knapp 30\% über dem Wert der USA mit neun Codierungen pro Text. Bei den Wertecodierungen wird die Differenz größer: mit 17 Wertecodierungen pro Text liegen Texte aus den USA zwar im Schnitt aller englischen Texte, aber Texte aus dem UK erreichen mit durchschnittlich 24 Wertecodierungen $41 \%$ mehr Codierungen als der Durchschnitt der englischen Texte.

\footnotetext{
${ }^{283}$ Das Land, in dem sich die Hochschule befindet, der der Autor angehört (vgl. 3.2.5).
} 


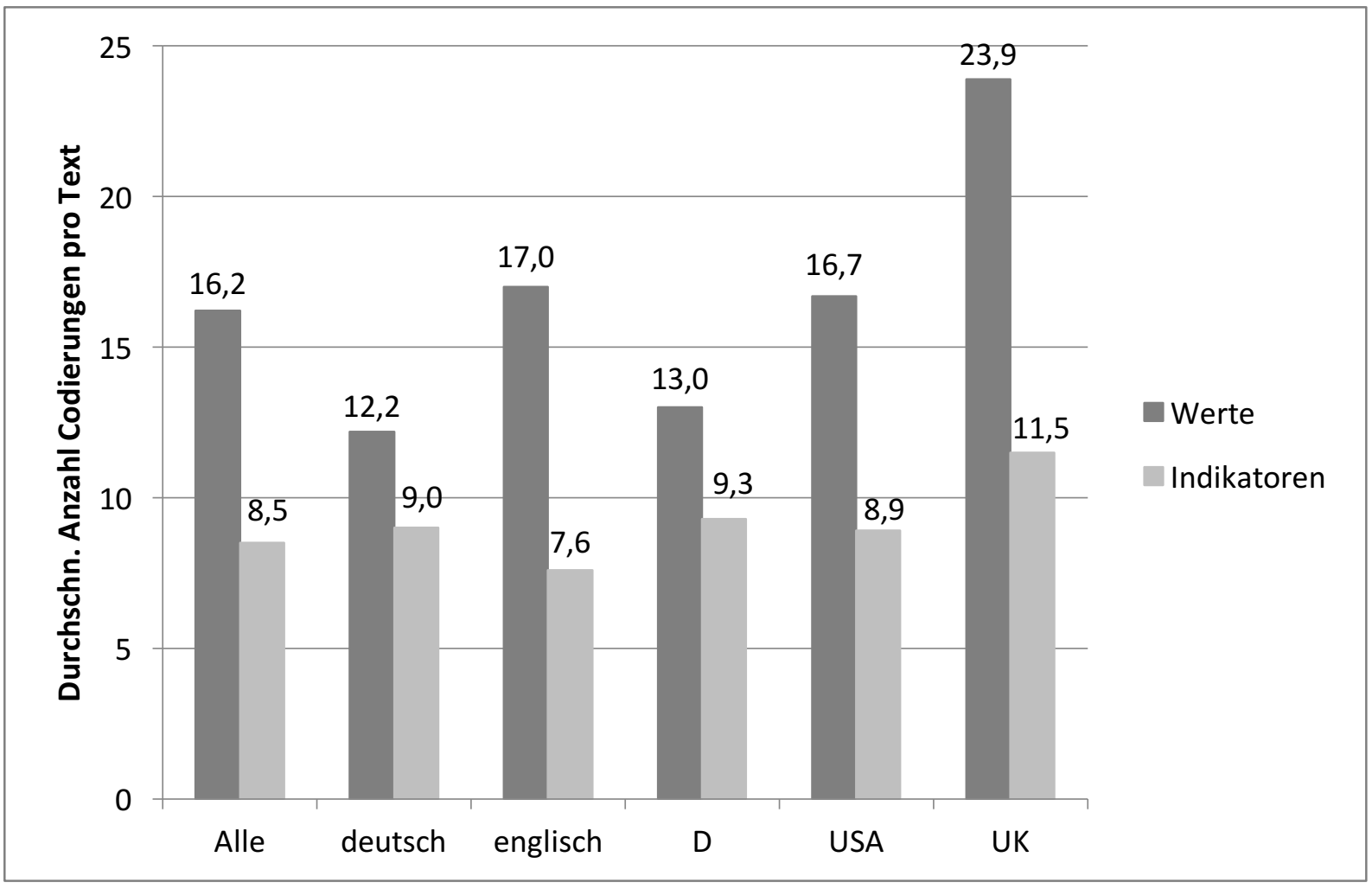

Abbildung 14: Durchschnittliche Anzahl Codierungen pro Text (nach Sprache bzw. Land)

Auch der Vergleich der Anzahl der Codierungen nach der Art des Mediums zeigt einige deutliche Unterschiede. Wie in Abbildung 15 dargestellt, weisen Artikel in Zeitschriften durchschnittlich 15 Wertecodierungen auf, Artikel in Sammelbänden dagegen nur $10 .^{284}$ Genau gegenläufig verhält sich die Anzahl der Indikatorcodierungen. In Zeitschriftenartikeln finden sich durchschnittlich sechs Indikatorcodierungen, während Beiträge in Sammelbänden im Schnitt 10 Indikatorcodierungen enthalten. Somit halten sich Werte- und Indikatorcodierungen in Sammelbänden die Waage. In Zeitschriften hingegen gibt es durchschnittlich 2,4-mal so viele Werte- wie Indikatorcodierungen. Um hier weiter zu differenzieren, sind die beiden Zeitschriften aus denen die meisten Texte stammen ebenfalls dargestellt. Die Anzahl der Indikatorcodierungen weicht vom Durchschnitt bei beiden Zeitschriften leicht nach oben ab. Die Anzahl der Wertecodierungen unterscheidet sich mit 19 deutlich vom Durchschnitt aller Zeitschriften (15). Des Weiteren ist die Auswertung für die beiden Hochschulen mit den meisten Beiträgen dargestellt. Sämtliche analysierten Texte der Universitäten Göttingen und Darmstadt sind Sammelbänden entnommen. Während jedoch die Anzahl der Codierungen von Werten und Indikatoren in Sammelbänden in etwa gleich hoch ist, zeigen die Beiträge beider Hochschulen mehr Indikatorcodierungen als Wertecodierungen. Insbesondere die Anzahl der Wertecodierungen in Darmstädter Texten liegt mit 13 im Vergleich zum Durch-

\footnotetext{
${ }^{284}$ Die Länge der Texte ist mit durchschnittlich 23,5 Seiten in Sammelbänden und 23,0 Seiten in Zeitschriften beinahe identisch. Die unterschiedliche durchschnittliche Anzahl der Wertecodierungen ist somit nicht auf die Textlänge zurückzuführen.
} 
schnitt aller Texte (neun) um 51\% höher und auch im Vergleich mit dem Durchschnitt in Sammelbänden (10) noch um 31\% höher.

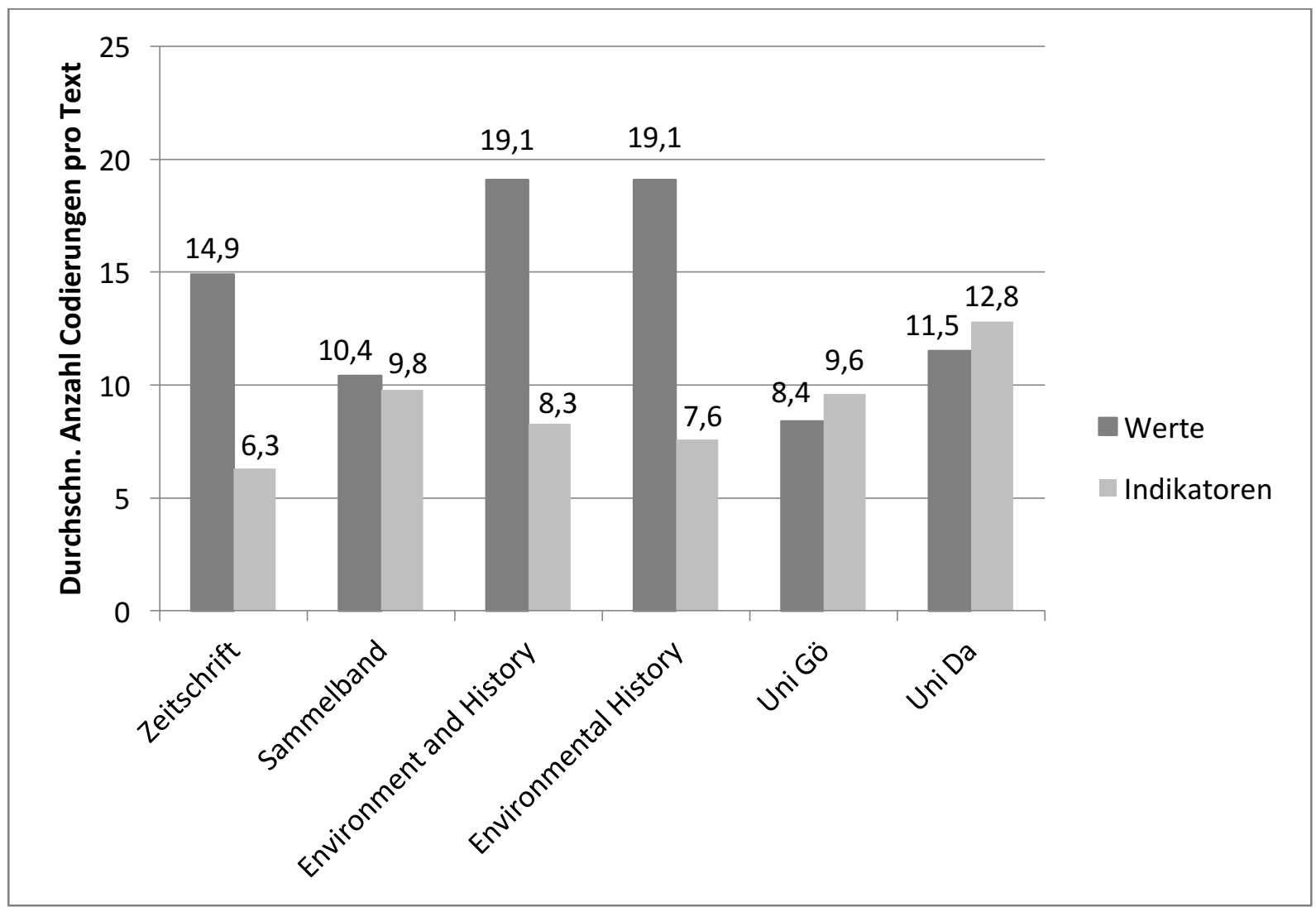

Abbildung 15: Anzahl Codierungen pro Text (nach Medium bzw. Universität)

Insgesamt lässt sich feststellen, dass in Zeitschriften publizierte Texte deutlich mehr Wertefundstellen (im Schnitt 15) aufweisen als Beiträge in Sammelbänden (im Schnitt 10). Andererseits ist eine geringere Anzahl an Indikatoren pro Zeitschriftentext (durchschnittlich sechs) gegenüber den Texten aus Sammelbänden (durchschnittlich 10) festzustellen.

Als letzten Vergleich von Indikatoren und Werten zeigt Abbildung 16 die Anzahl der Wertecodierungen im Abhängigkeit von der Nennung des Indikators „Umweltschaden“. Insgesamt ist kein direkter Zusammenhang erkennbar, vielmehr wird deutlich, dass die explizite Benennung eines Phänomens als „Umweltschaden“ weder einen Rückschluss auf die Anzahl der Wertefundstellen noch auf die vorgefundenen Werte zulässt. Wird die Bezeichnung „Umweltschaden“ allerdings nicht im Text verwendet weisen bestimmte Werte eine deutlich höhere Anzahl an Wertecodierungen auf. Die Werte „Ökonomie“, „Zuhause, Eigentum“ und „Tod“ zeigen in diesem Fall eine 4-mal so hohe Anzahl an Codierungen und „Schönheit der Natur" eine 6-mal so hohe. Die Anzahl bei „Tiere“ und „Unberührte Natur“ ist 15-mal höher, bei „Infrastruktur“ sogar 26mal höher und bei „Erholung, Freizeit, Komfort“ 28mal höher. Dagegen weisen manche Werte mehr Codierungen auf, wenn der Indikator 
„Umweltschaden“ genannt wird, u. a. „Wasser“, „Nachhaltigkeit“, „Imageschaden“, „Hunger“ und "Luft".

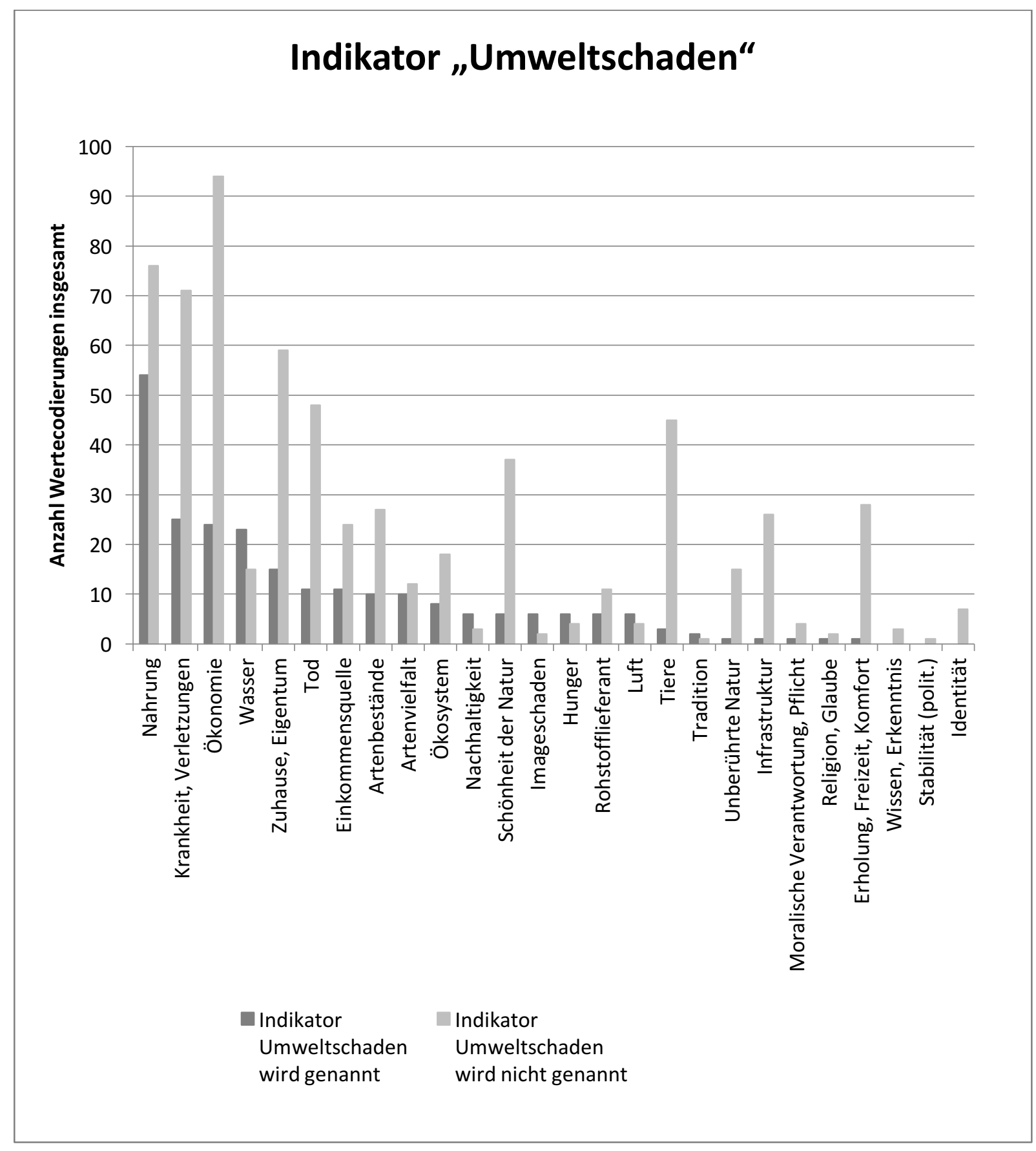

Abbildung 16: Anzahl Wertecodierungen und der Indikator „Umweltschaden“

Ein bislang noch nicht untersuchter Aspekt betrifft die Frage wie konkret eine Fundstelle den Schaden bzw. die Beeinträchtigung des Wertes beschreibt. Der folgende Abschnitt analysiert daher die Wertefundstellen hinsichtlich quantifizierender Attribute im unmittelbaren Kontext der Fundstelle und soll die Frage beantworten, welche Werte quantifiziert werden und wie häufig dies geschieht. Hierbei wird jegliche Konkretisierung erfasst, die je nach Wert sehr 
unterschiedlich ausfallen kann (z.B. Anzahl der getöteten Personen, monetäre Kosten der Beseitigung des Schadens, Fläche der zerstörten Felder). Um trotzdem eine Vergleichbarkeit zu gewährleisten und zu berücksichtigen, dass z.T. zwar keine konkreten quantifizierenden Angaben gemacht wurden, jedoch zumindest eine vage beschreibende Einordnung der Schadensgröße erfolgte, wurden drei Kategorien gewählt:

„Ja“ Quantifizierung vorhanden,

„Vage“ nur vage Quantifizierung vorhanden,

„Nein“ $\quad$ keine Quantifizierung vorhanden.

Am Beispiel der Werte Ökonomie und Tod sollen diese Kategorien kurz verdeutlicht werden $^{285}$ :

Ökonomie Tod

\begin{tabular}{lll}
\hline Ja & "more than $\$ 2$ million in repairs" & "took 89 lives“ \\
Vage & "massive economic damage“ & "massive death toll" \\
Nein & "economic losses" & "an increase in heat related deaths"
\end{tabular}

Abbildung 17 zeigt eine Auswertung über alle Wertecodierungen nach Anzahl der Codierungen. Nur 15\% aller Fundstellen beinhalten demnach quantifizierende Attribute und weitere $8 \%$ weisen zumindest eine vage Quantifizierung auf. $77 \%$ hingegen zeigen keine Quantifizierung.

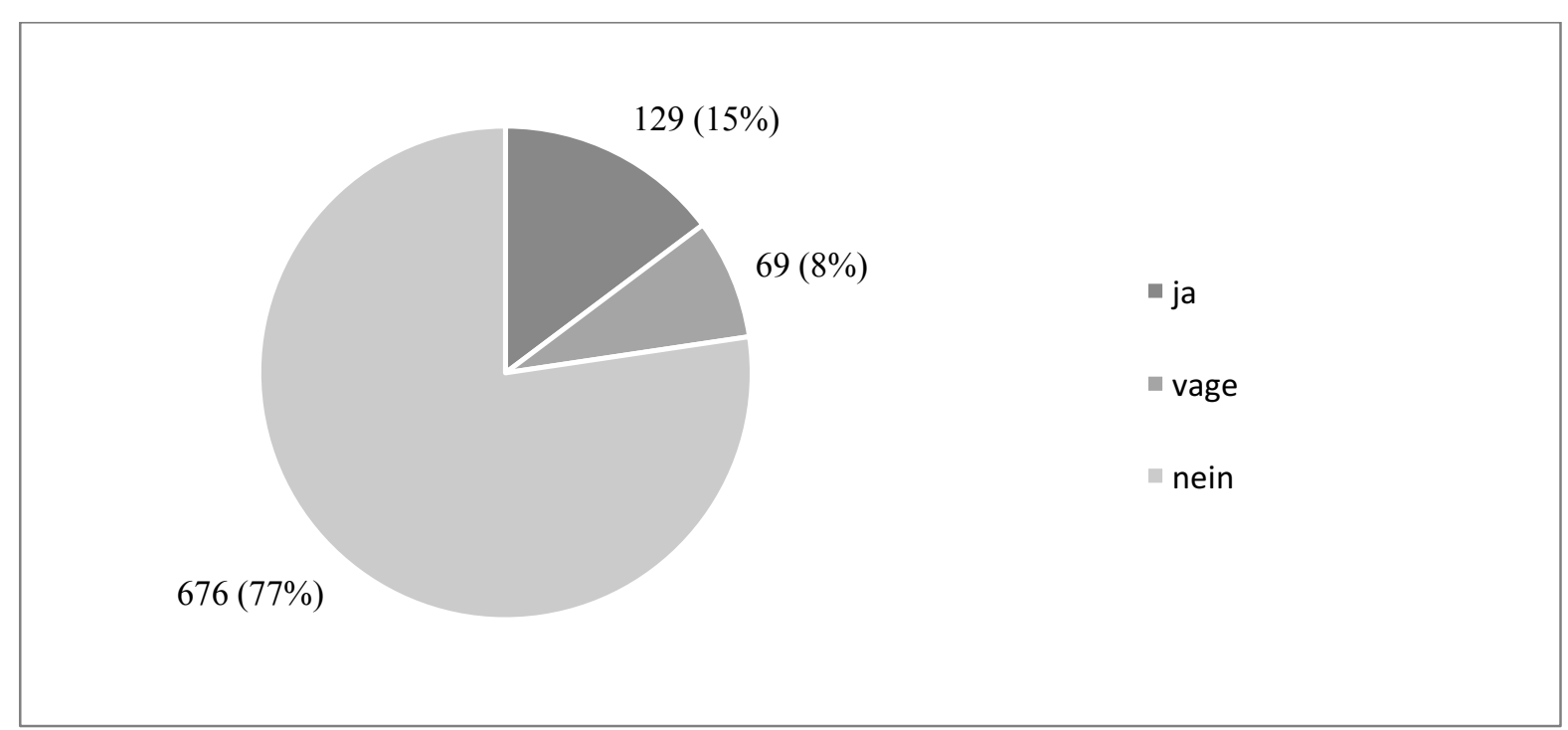

Abbildung 17: Quantifizierte Wertecodierungen (nach Anzahl Codierungen)

Betrachtet man nun die Werte im Einzelnen, weisen folgende Werte keine Quantifizierung oder maximal eine Fundstelle mit Quantifizierung auf:

285 Quellen Ökonomie (Name Dokument in MAXQDA; Zeilennummer in MAXQDA): deKraker 2013;410, Aguirre 2012;54, Castonguay 2007;117. Quellen Tod: Cao 2012;95, Mann 1995;120, Bruno 2013;9. 
Keine Quantifizierung:

max. eine Quantifizierung:

Stabilität (polit.)

Hunger

Religion, Glaube

Unberührte Natur

Tradition

Rohstofflieferant

Wissen, Erkenntnis

Artenvielfalt

Moralische Verantwortung, Pflicht

Identität

Imageschaden

Nachhaltigkeit

Ökosystem

Dies ist in der Art dieser Werte begründet, die bis auf die Werte Rohstofflieferant und Hunger zu den immateriellen Werten gehören. Eine Quantifizierung dieser Werteart erscheint schwierig bis nicht möglich. Insgesamt gibt es für die o.g. Werte ohne Quantifizierungen 65 Fundstellen (7\% aller Wertecodierungen) in den analysierten Dokumenten.

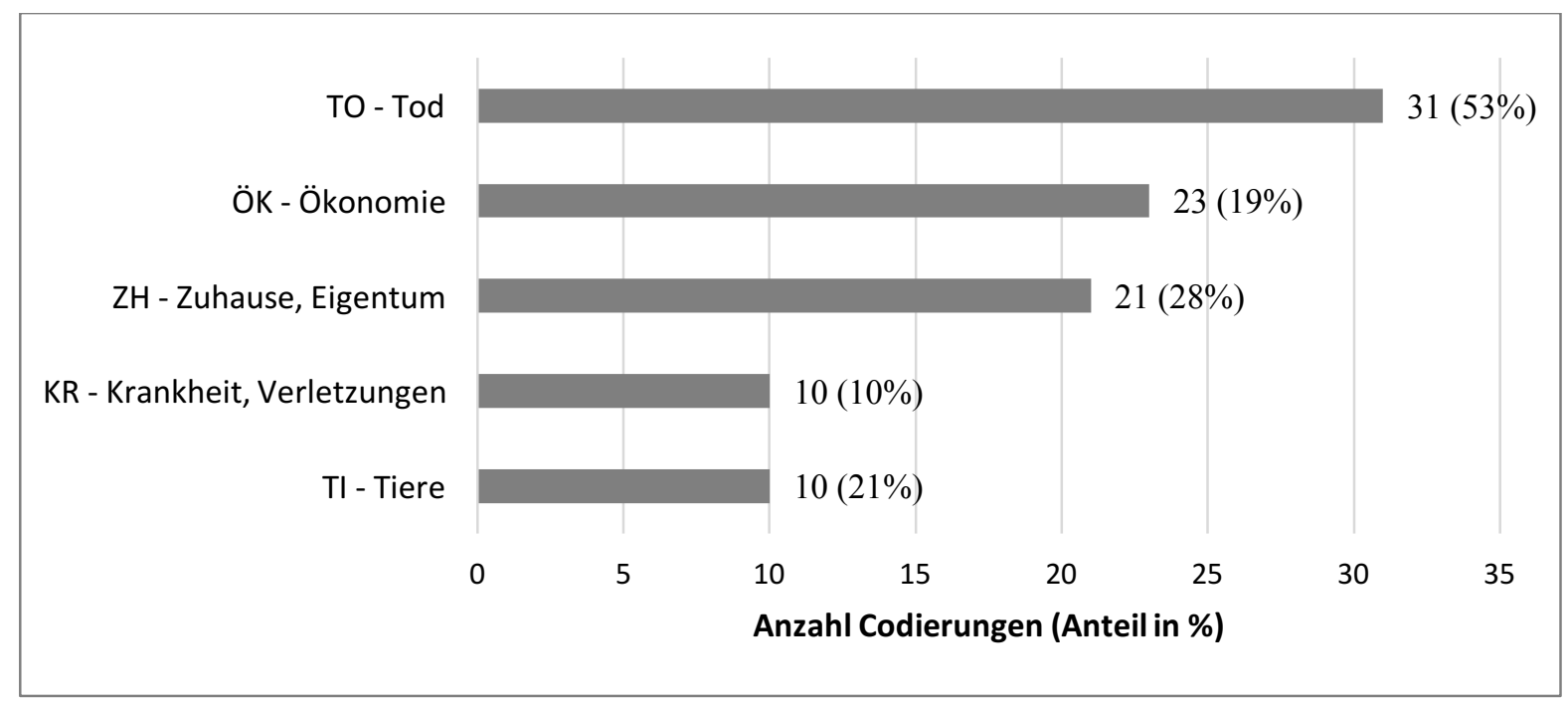

Abbildung 18: Quantifizierte Werte (nach Anzahl Codierungen)

Abbildung 18 zeigt die am häufigsten quantifizierten Werte, die mindestens zehn quantifizierende Fundstellen aufweisen. Selbst bei dem Wert Tod, der mit 31 quantifizierenden Fundstellen der am häufigsten quantifizierte Wert ist, liegt der Anteil an quantifizierenden Wertecodierungen nur bei $53 \%$. Die zweit- und drittplatzierten Werte Ökonomie und Zuhause, Eigentum erreichen sogar nur Quoten von 19\% bzw. 28\%. Dass die Werte von denen anzunehmen ist, dass sie im Vergleich zu den immateriellen Werten (s.o.) eindeutiger zu quantifizieren sein sollten, trotzdem eher selten quantifiziert werden, verwundert.

Bei der Auswertung nach Anteil Dokumente wie in Abbildung 19 dargestellt, zeigt sich, dass sich die relativ wenigen quantifizierenden Textstellen über viele Dokumente verteilen. So 
enthalten 61\% aller untersuchten Dokumente mindestens eine Quantifizierung und weitere $15 \%$ beinhalten zumindest eine vage Quantifizierung. Bleiben allerdings $24 \%$ die keine Quantifizierungen enthalten.

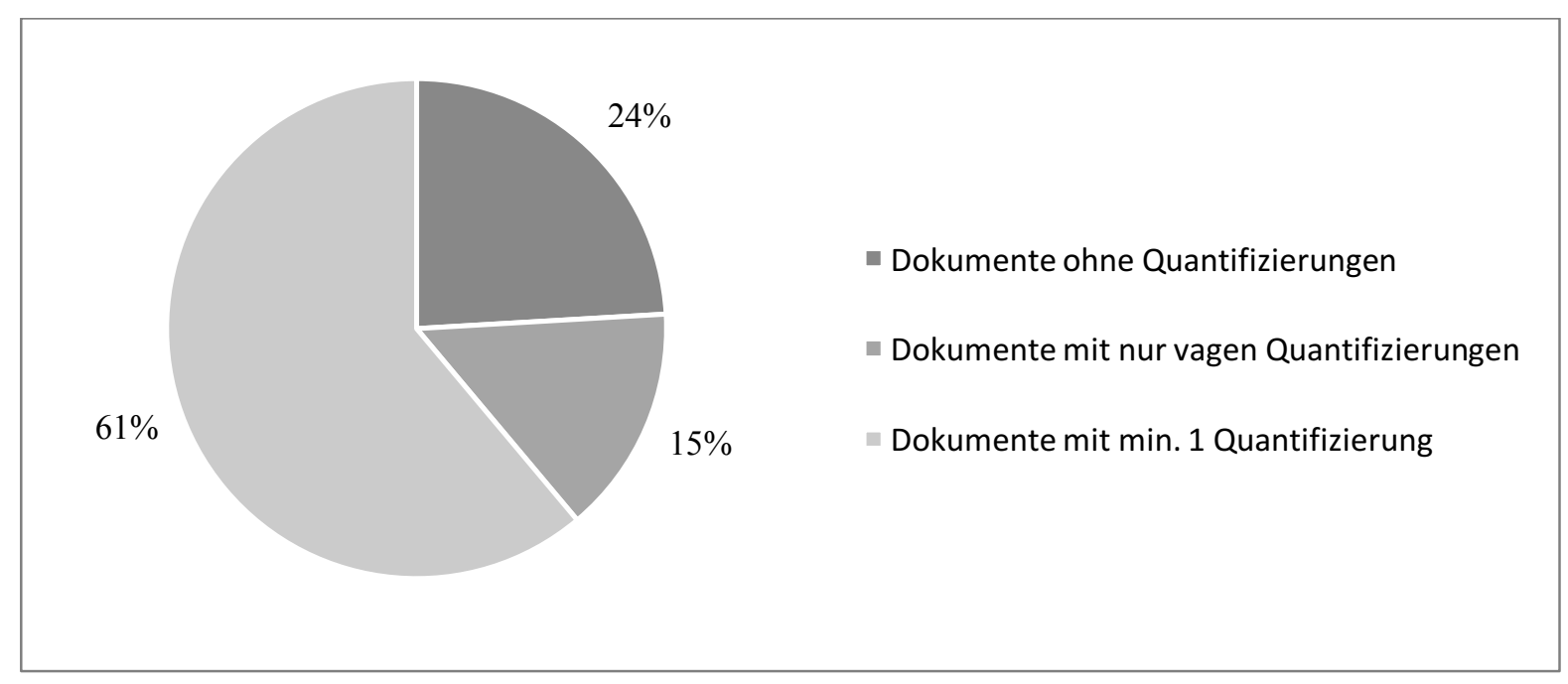

Abbildung 19: Quantifizierte Wertecodierungen (nach Anteil Dokumente)

\subsection{Qualitative Beschreibung der untersuchten Texte}

Die untersuchten Texte enthalten verschiedenste Themen sowie eine angemessene Verteilung hinsichtlich Schadensdauer und -ausdehnung und Ereignisdauer und -ausdehnung. Der Stichprobenumfang liegt mit 54 Texten im empfohlenen Rahmen ${ }^{286}$ der durch den Abschluss der induktiven Kategorienbildung nach 18 Texten bestimmt wurde (Mindestumfang 36 Texte). Abbildung 20 präsentiert zunächst eine Übersicht aller untersuchten Texte mit Stichworten zu den behandelten Themen, Schäden und Ursachen. Die dann folgenden Abbildungen zeigen Vergleiche von Ereignisdauer und Ereignisfläche sowie Schadensdauer und Schadensfläche.

${ }^{286}$ Vgl. Fußnote 264, S. 64. 


\begin{tabular}{|c|c|c|c|}
\hline $\begin{array}{l}\text { Verfasser, } \\
\text { Erscheinungsjahr }\end{array}$ & Stichwort zum Thema & Stichwort zum Schaden & Ursache \\
\hline Aguirre_2012 & Erdbeben & Tote, Sachschaden & Naturkatastrophe \\
\hline Amate 2013 & Bodenerosion & Bodenverlust & Mensch \\
\hline Bankoff_2007 & Überflutungen & Getötete Haus-/Nutztiere & Naturkatastrophe \\
\hline Bayerl_1989 & Umweltverschmutzung & Trinkwasser-/Luftverschmutzung & Mensch \\
\hline Bayerl_2009 & $\begin{array}{l}\text { Umweltverschmutzung durch } \\
\text { Planwirtschaft }\end{array}$ & Umweltverschmutzung & Mensch \\
\hline Beattie_2003 & Abholzung & $\begin{array}{l}\text { Bodenerosion, Überflutungen, } \\
\text { Klimaveränderung }\end{array}$ & Mensch \\
\hline Bodner_2010 & Felsstürze & Arbeitsplätze, Evakuierung & Naturkatastrophe \\
\hline Bowlus_1988 & Abholzung, Pest, Klima & Tote, Wirtschaft & Krankheit, Mensch \\
\hline Brantz_2011 & Tierseuche & $\begin{array}{l}\text { Tote Rinder, finanz.Verluste, } \\
\text { Nahrungsknappheit }\end{array}$ & Tierseuche \\
\hline Bruno_2013 & Lawine & Tote & Naturkatastrophe \\
\hline Buchholz_2012 & Umnutzung als Ackerland & $\begin{array}{l}\text { Lebensraum- und } \\
\text { Bodenverschlechterung }\end{array}$ & Mensch \\
\hline Buhs_2002 & Pestizide & $\begin{array}{l}\text { Verlust von Nutztieren (Rinder), } \\
\text { Vergiftung von Nahrungsmitteln }\end{array}$ & Mensch \\
\hline Cao_2012 & Vulkanausbruch & $\begin{array}{l}\text { Zerstörung Lebensraum, } \\
\text { Klimaveränderung, Ernteausfälle }\end{array}$ & Naturkatastrophe \\
\hline Carey_2007 & Gletscherschmelze & $\begin{array}{l}\text { Kein Wasser vor Ort; Meerhöhe } \\
\text { steigt }\end{array}$ & Mensch \\
\hline Castonguay_2007 & Überflutungen & Eigentum, Produktionsausfälle, Tote & Mensch, Naturkatastrophe \\
\hline Crook_2002 & Abholzung, Überflutung & $\begin{array}{l}\text { Mangel an Holz, Zerstörung durch } \\
\text { Überflutung }\end{array}$ & Mensch, Naturkatastrophe \\
\hline Cyffka_2007 & Umnutzung als Bergbau & $\begin{array}{l}\text { Verlust Schönheit der Natur, Verlust } \\
\text { menschl. Lebensraum }\end{array}$ & Mensch \\
\hline Daley_2008 & Korallensammeln & (teilweise) Zerstörung des Riffs & Mensch \\
\hline de Kraker_2013 & Stürme & Zerstörung, Überflutung & Naturkatastrophe \\
\hline Denning_2014 & Skifahren & Keine unberührte Natur mehr & Mensch \\
\hline Engels_2009 & Sturmflut & Tote, Sachschaden & Naturkatastrophe \\
\hline Griggs_2007 & Abholzung, Zuckerrohranbau & Artenvielfalt/-bestände, Ökosystem & Mensch \\
\hline Huff_2010 & Klimaanomalie & Ernteausfall & Naturkatastrophe \\
\hline Josephson_2010 & Kalter Krieg & Krankheit, Nahrungsmittelproduktion & Mensch \\
\hline Knopf_2010 & Bodenübernutzung & Bodenverlust & Mensch \\
\hline Kraft_2013 & Tschernobyl & Radioaktive Verstrahlung & Mensch \\
\hline Latorre_2001 & Abholzung & Austrocknung, Wüstenbildung & Mensch \\
\hline Mann_1995 & Abholzung & $\begin{array}{l}\text { Klima, Bodenverschlechterung, } \\
\text { Wasserknappheit }\end{array}$ & Mensch \\
\hline Mathews_2003 & Waldbrände & Ökonomie & Naturkatastrophe \\
\hline Mc Farlane_2012 & Umweltverschmutzung & Gestank & Mensch \\
\hline McClenachan_2013 & Fischen & Reduzierte Fischpopulation & Mensch \\
\hline Muscolino_2011 & Flüchtlingsmigration & Ausbeutung Acker/Wald & Mensch \\
\hline Obertreis_2013 & Verlandung & Gesundheit, Fischerei & Mensch, Naturkatastrophe \\
\hline Perri_2009 & Perlensuche & $\begin{array}{l}\text { Zerstörung, Ausbeutung der } \\
\text { Austernbänke }\end{array}$ & Mensch \\
\hline Pritchard_2012 & Fukushima & Verstrahlung & Mensch, Naturkatastrophe \\
\hline Rohr_2003 & Erdbeben & Zerstörte Häuser, Tote & Naturkatastrophe \\
\hline Rohr_2007 & Tierplagen & Lebensraum, Nahrung & Naturkatastrophe \\
\hline Romero_2002 & Tierjagd & $\begin{array}{l}\text { Artenbestände reduziert bzw. } \\
\text { ausgerottet }\end{array}$ & Mensch \\
\hline Ross_2014 & Zinngewinnung & Boden- und Landschaftszerstörung & Mensch \\
\hline Schenk_2010 & Überflutungen, Erdbeben & Tote, Sachschäden & Naturkatastrophe \\
\hline Scheringer_2012 & Chemikalienfreisetzung & $\begin{array}{l}\text { Gesundheitliche Schädigung des } \\
\text { Menschen, Schädigung Flora und } \\
\text { Fauna }\end{array}$ & Mensch \\
\hline Schott_1997 & Energieverbrauch & Gesundheit, Landschaft & Mensch \\
\hline Schott_2002 & Feuer, Cholera, Flut & Gesundheit, Besitz & Krankheit, Naturkatastrophe \\
\hline Schulze_2013 & Industrialisierung & Wasser, Luft, Gesundheit & Mensch \\
\hline Sheail_2013 & Pestizide & Gesundheit & Mensch \\
\hline Shulmann_2010 & Kalter Krieg & Gesundheit, Ökonomie & Mensch \\
\hline Singh_2000 & $\begin{array}{l}\text { Kommerzialisierung } \\
\text { Landwirtschaft }\end{array}$ & Bodenerosion & Mensch \\
\hline Slavin_2014 & Krieg & Ackerzerstörung, Rinderverlust & Mensch \\
\hline Smith_2012 & Hurrikan & Eigentum, Tote & Naturkatastrophe \\
\hline Studnicki_2010 & Bergbau, Abholzung & Abgeholzter Wald & Mensch \\
\hline Swanson_2012 & Tourismus & Landschaft, Artenvielfalt & Mensch \\
\hline Tully_2009 & Kautschukgewinnung & Regenwaldverlust & Mensch \\
\hline Völkel_2014 & Bergbau & Wasser, Boden, Gesundheit & Mensch \\
\hline Worster_1988 & Dürre & Winderosion/Staubstürme & Naturkatastrophe \\
\hline
\end{tabular}


Die Art der den Schäden zugrunde liegenden Ereignisse ist sehr unterschiedlich und reicht von Erdbeben, Stürmen, Lawinen und Vulkanausbrüchen über Dürren, Misswirtschaft, Tierplagen und Rohstoffgewinnung bis zu nuklearen Zwischenfällen. Dementsprechend unterschiedlich ist auch der jeweils betrachtete Ereigniszeitraum, der von einigen Sekunden bei Erdbeben bis zu mehreren Jahrhunderten, z. B. bei der Rohstoffgewinnung, reicht. Bei 26\% der analysierten Texte beträgt die Ereignisdauer weniger als $1 \mathrm{Jahr}$, bei weiteren $48 \%$ beträgt sie von 1 bis 100 Jahren und bei den restlichen $26 \%$ ist sie größer als 100 Jahre. Nicht minder unterschiedlich ist die Art und Dauer der zugehörigen Schäden, die von kurzzeitigen Komfortbeeinträchtigungen (z. B. durch unangenehme Gerüche) über Dezimierung von Arten bis zu dauerhafter Zerstörung von Natur und menschlichen Todesopfern ${ }^{287}$ reichen.

Während die Art der Schäden in den Abhandlungen beschrieben wird, sind die Ausführungen zur Dauer nicht immer hinreichend. Oftmals konnte der Schadenszeitraum aus den Texten abgeleitet werden, bei einigen Aufsätzen musste jedoch eine Schätzung vorgenommen werden.

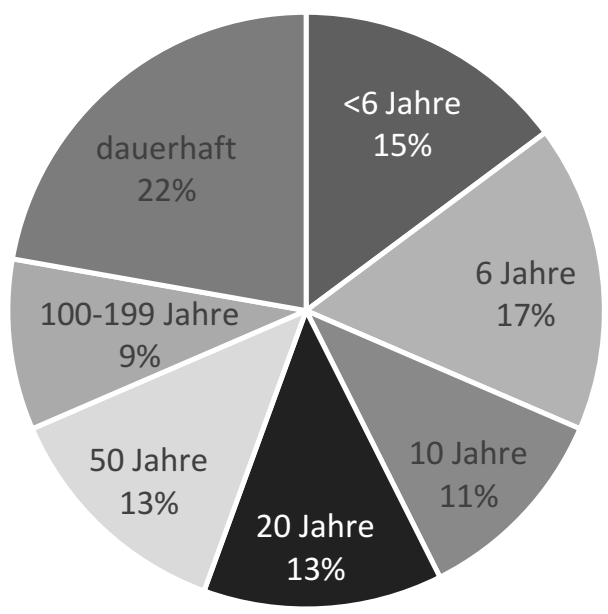

\section{Abbildung 21: Verteilung der Schadensdauer}

Es ergibt sich folgende Verteilung, wie in Abbildung 21 dargestellt: Bei 32\% der Dokumente liegt die Schadensdauer unter 10 Jahren, bei $37 \%$ beträgt sie von 10 bis 99 Jahren, bei $9 \%$ liegt die Schadensdauer von 100 bis 199 Jahren und bei 22\% ist der Schaden als dauerhaft ${ }^{288} \mathrm{zu}$ betrachten. ${ }^{289}$ Sowohl bezüglich Ereignis- als auch Schadensdauer weisen die ausgewählten Texte also eine angemessene Verteilung auf.

\footnotetext{
${ }^{287}$ Diese Aufzählung soll durchaus die Bandbreite des Schadensausmaßes verdeutlichen, ist aber keinesfalls als Wertung oder Rangfolge der Schäden zu interpretieren.

288 „Dauerhaft“ wird hier als Zeitraum von mindestens 200 Jahren definiert.
} 


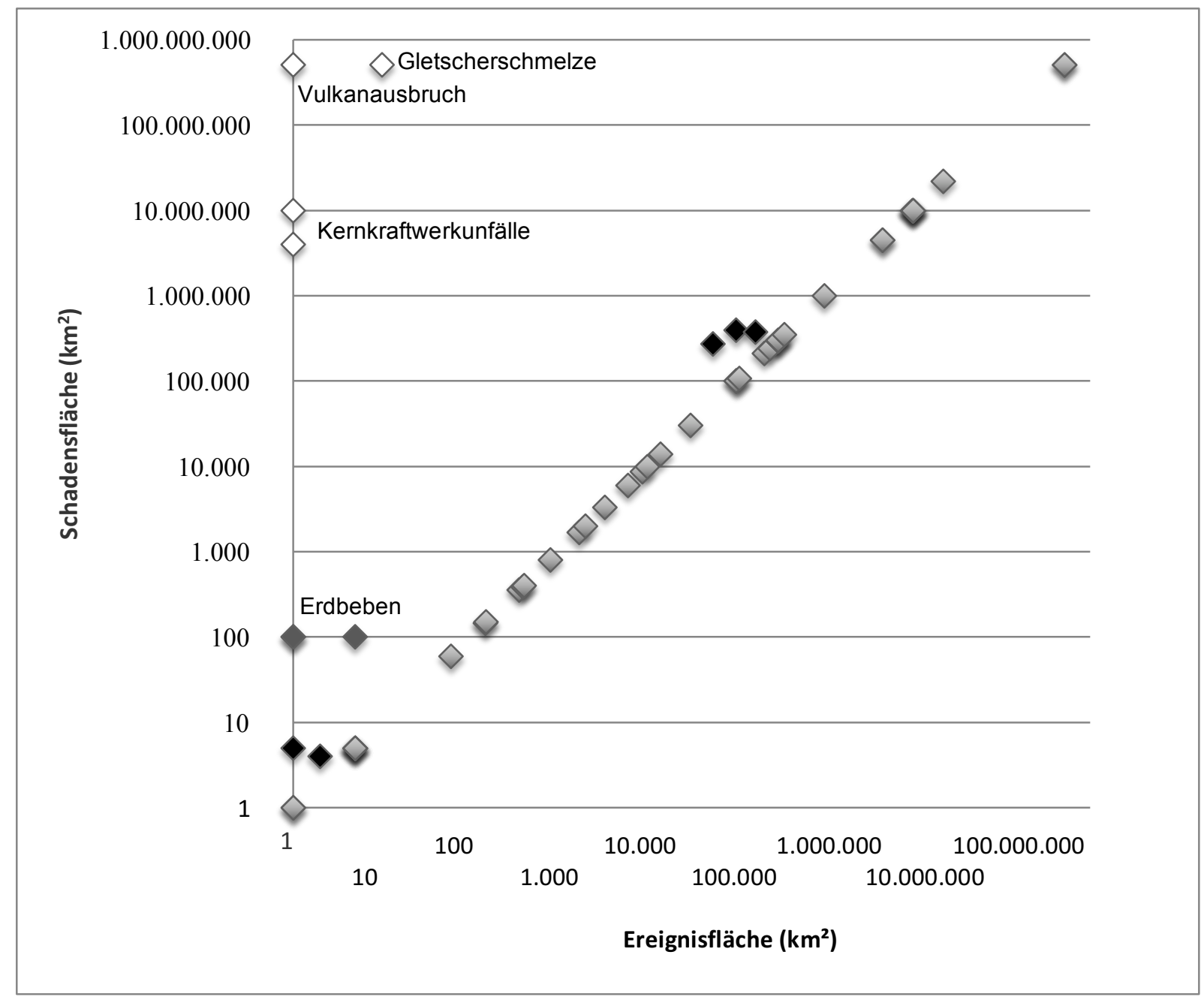

\section{Abbildung 22: Ereignis- und Schadensausdehnung (Fläche) ${ }^{290}$}

Neben der Ereignis- und Schadensdauer wurden auch die betroffenen Ereignis- und Schadensflächen erfasst. Abbildung 22 zeigt die Ereignisfläche im Vergleich zur Schadensfläche. Aufgrund der sehr unterschiedlichen Flächengrößen (von lokal bis global, d. h. von $1 \mathrm{~km}^{2}$ bis $510.000 .000 \mathrm{~km}^{2}$ ) wurde für die Darstellung eine logarithmische Skalierung gewählt. Bei den meisten Texten sind Ereignis- und Schadensfläche identisch bzw. nahezu

\footnotetext{
${ }^{289}$ Für die Auswertungen wurden folgende Glättungen in den Daten vorgenommen: Wenn keine konkrete Dauer sondern ein Zeitfenster angegeben wurde, wurde der Median verwendet; z. B. wurde aus 1-10 Jahre 5,5 Jahre. Der maximale Zeitraum für ein Ereignis wurde auf 500 Jahre festgesetzt. Die maximale Schadensdauer wurde mit 200 Jahren definiert; d. h. ein dauerhafter, aber zeitlich nicht näher quantifizierter Schaden wird mit einer Dauer von 200 Jahren bewertet. Wenn die Schadensdauer noch offen ist, d.h. der Schaden bereits begonnen hat, aber unklar ist, wie lange er noch andauert, wurde die aktuelle Schadensdauer unterstellt. Bei langen Ereigniszeiträumen kann die Schadensdauer auch während des Ereignisses beginnen (Schaden parallel zum Ereignis), meist beginnt der Schaden aber nach dem Ereignis. Bei einer nicht näher definierten Fläche (Ereignis und Schaden) wird die Größe entweder pauschal gesetzt (lokal $=1 \mathrm{~km}^{2}$, national/international = entsprechend der Landesgröße des behandelten Landes, global $=510.000 .000 \mathrm{~km}^{2}$ ) oder qualifiziert geschätzt (z.B. bei Küstenstürmen: Länge der Küste * 10 km Breite).

290 Geringfügige Abweichungen (zwei- bis vierfache Schadensfläche im Vergleich zur Ereignisfläche) als schwarze Punkte dargestellt, deutliche Abweichungen (20- bis 100 fache Fläche) als dunkelgraue Punkte und extreme Abweichungen (4.000.000- bis 510.000.000fache Fläche) als weiße Punkte. Die extremen Abweichungen beziehen sich auf Texte zu Vulkanausbruch, Gletscherschmelze und Kernkraftwerkunfällen. Deutliche Abweichungen stammen aus Texten zu Erdbeben.
} 
identisch, dies trifft auf $78 \%$ der Texte zu. Bei den anderen Dokumenten gibt es unterschiedlich stark ausgeprägte Abweichungen, jedoch ist die Schadensfläche stets größer als die Ereignisfläche. Bei fünf Texten $(9 \%)$ ist die Schadensfläche geringfügig größer als die Ereignisfläche, bei weiteren drei Texten (6\%) zeigt sich eine deutliche Abweichung und bei vier Texten (7\%) weicht die Schadensfläche extrem von der Ereignisfläche ab.

Beim Vergleich von Ereignisdauer und Ereignisausdehnung ist dagegen kein mathematischer Zusammenhang mehr sichtbar. Abbildung 23 vergleicht die Dauer eines Ereignisses mit dessen räumlicher Ausdehnung. Auch hier wurde für die Darstellung der Fläche ${ }^{291}$ eine logarithmische Skalierung gewählt.

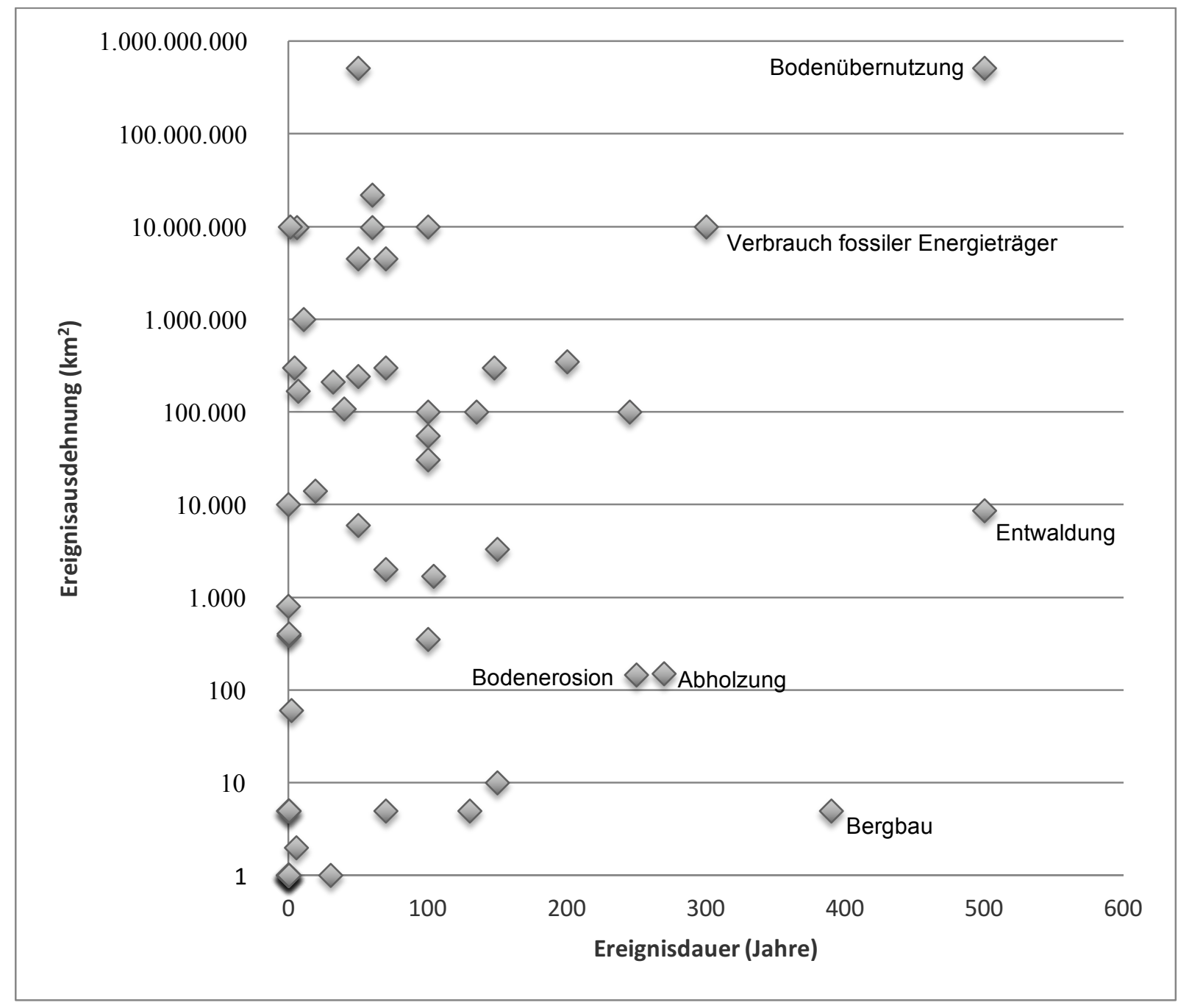

\section{Abbildung 23: Ereignisdauer und -ausdehnung}

Zunächst lässt sich feststellen, dass die Verteilung eher breiter gestreut ist und zwar sowohl hinsichtlich der Ereignisdauer als auch bezüglich der Ereignisausdehnung. Es ist eine Häufung der Punkte im Ausdehnungsbereich $1.000-10.000 .000 \mathrm{~km}^{2}$ und zwischen fast 0 bis

291 Zur leichteren Einordnung der Flächengrößen können die ungefähren Flächen von Deutschland mit $350.000 \mathrm{~km}^{2}$, Europa mit $10.000 .000 \mathrm{~km}^{2}$ und der gesamten Erdoberfläche mit $510.000 .000 \mathrm{~km}^{2}$ als Orientierungshilfe dienen. 
etwa 150 Jahren Ereignisdauer zu sehen. 47 Texte (87\%) betrachten eine Ereignisdauer von bis zu 150 Jahren. 24 davon beschreiben eine Ereignisfläche zwischen $1.000 \mathrm{~km}^{2}$ und 10.000.000 km². Damit liegen 44\% aller analysierten Dokumente in diesem Bereich. Punkte die besonders abweichen, sind mit einem Stichwort zum untersuchten Phänomen beschriftet, um die Kombination von Ereignisdauer und -fläche besser nachvollziehen zu können.

In diesem Zusammenhang ist auch ein Blick auf die Ursachen der in den Dokumenten beschriebenen Schäden bzw. der Kategorie des zugrunde liegenden Ereignisses angebracht. ${ }^{292}$ Abbildung 24 zeigt, dass $61 \%$ der untersuchten Texte von Menschen verursachte Schäden behandeln, während Naturkatastrophen nur in $26 \%$ der Texte die Ursache der Schäden sind. Die meisten Texte untersuchen also Schäden, die auf das Einwirken des Menschen auf die Natur zurückzuführen sind. Umweltgeschichte befasst sich mit den Wechselwirkungen zwischen Mensch und Natur. Dass die Mehrzahl der analysierten Texte Wechselwirkungen untersucht, die mit dem Handeln des Menschen beginnen, verstärkt den Eindruck, dass ein anthropozentrischer Ansatz dominiert.

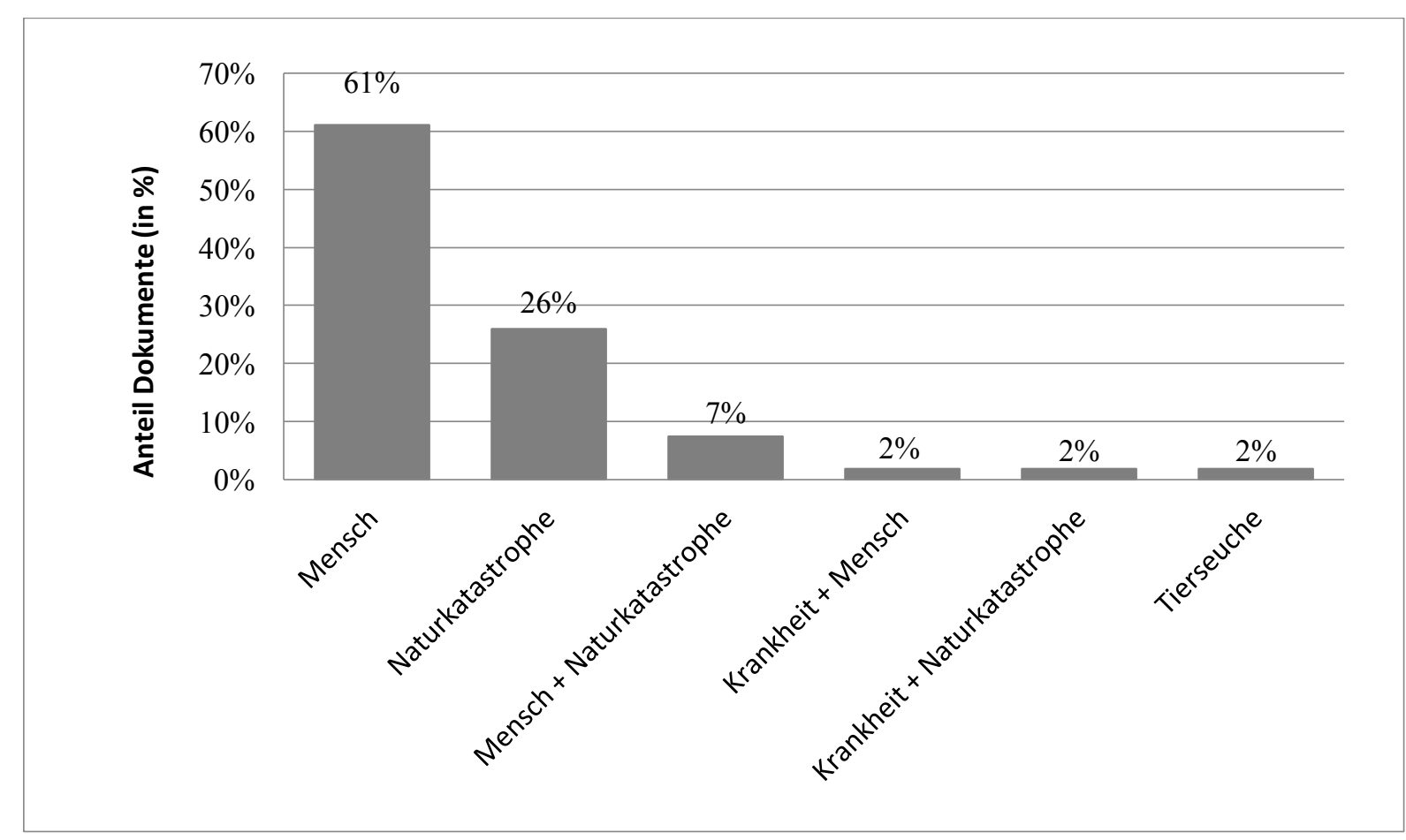

\section{Abbildung 24: Schadensursachen (nach Anteil Dokumente)}

Die folgende Abbildung ergänzt die Ereignisdauer und -fläche (Abbildung 23) um die Schadensdauer. Dafür entspricht in Abbildung 25 die Größe der Blasen der Schadensdauer (1-200 Jahre). In dieser Darstellung lässt sich ein Zusammenhang zwischen Ereignis- und Schadensdauer erkennen. Je länger die Ereignisdauer ist, desto seltener sind kurze

\footnotetext{
${ }^{292}$ Vgl. Abbildung 20: Themenübersicht der untersuchten Texte, S. 86.
} 
Schadenzeiträume. Des Weiteren fällt auf, dass eine kurze Ereignisdauer sowohl kurze als auch längerfristige Schäden verursachen kann und die Ereignisfläche gleichzeitig die gesamte Bandbreite von lokal bis global umfassen kann.

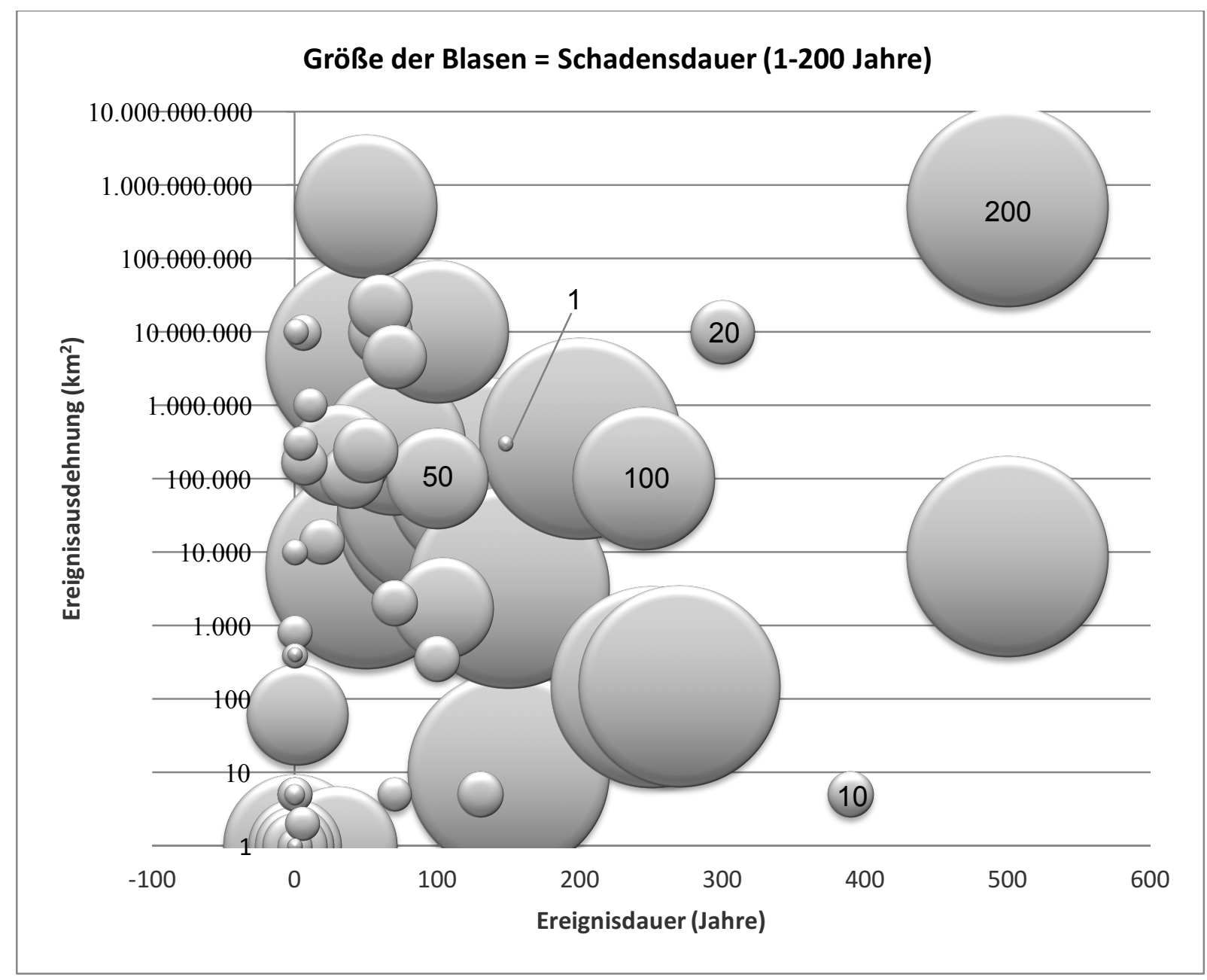

Abbildung 25: Ereignisdauer/-ausdehnung und Schadensdauer

Für gewöhnlich ist eine wissenschaftliche Abhandlung gut strukturiert; $d$. h. wenn bewusst eine Wertung dargelegt wird, sollte sich dies anhand von Wertecodierungen in einem begrenzten Bereich zeigen, jedoch nicht verstreut über den gesamten Text. Wenn die Codierungen jedoch über das ganze Dokument verteilt sind, spricht das meines Erachtens dafür, dass die markierten Werte eher unbewusst in den Text eingeflossen sind. Dies lässt sich mithilfe von Dokumentenporträts sichtbar machen. Hierbei wird der gesamte Text innerhalb bestimmter Abmessungen dargestellt, aber anstatt des Textes sind nur die codierten Textstellen farbig markiert dargestellt. Abbildung 26 zeigt zwei Beispiele. Die roten Punkte stehen für Indikatorcodierungen, die grünen für Wertecodierungen. Die Farbintensität nimmt mit der Länge des markierten Textes zu. ${ }^{293}$ Beim Vergleich der Dokumentenporträts

\footnotetext{
${ }^{293}$ Nur ein Wort codiert: schwache Färbung, eine ganze Zeile codiert: starke Färbung.
} 
der untersuchten Texte zeigt sich, dass bei der Mehrheit der Dokumente (69\%) die Codierungen über das gesamte Dokument verteilt sind, so wie bei dem Beispiel in Abbildung 26 auf der linken Seite. Bei weiteren $26 \%$ ist bei der Verteilung ein Schwerpunkt zu erkennen (siehe Abbildung 26 auf der rechten Seite), z. B. Codierungen nur in der ersten Hälfte des Dokuments und in 5\% der Fälle handelt es sich eher um punktuelle Codierungen, d.h. die Codierungen sind räumlich eng begrenzt und nicht sehr zahlreich. Bei der Mehrheit der untersuchten Texte verteilen sich die Wertecodierungen also über den gesamten Text, $d . h$. die Bewertungen fließen wahrscheinlich zum großen Teil unbewusst ein.

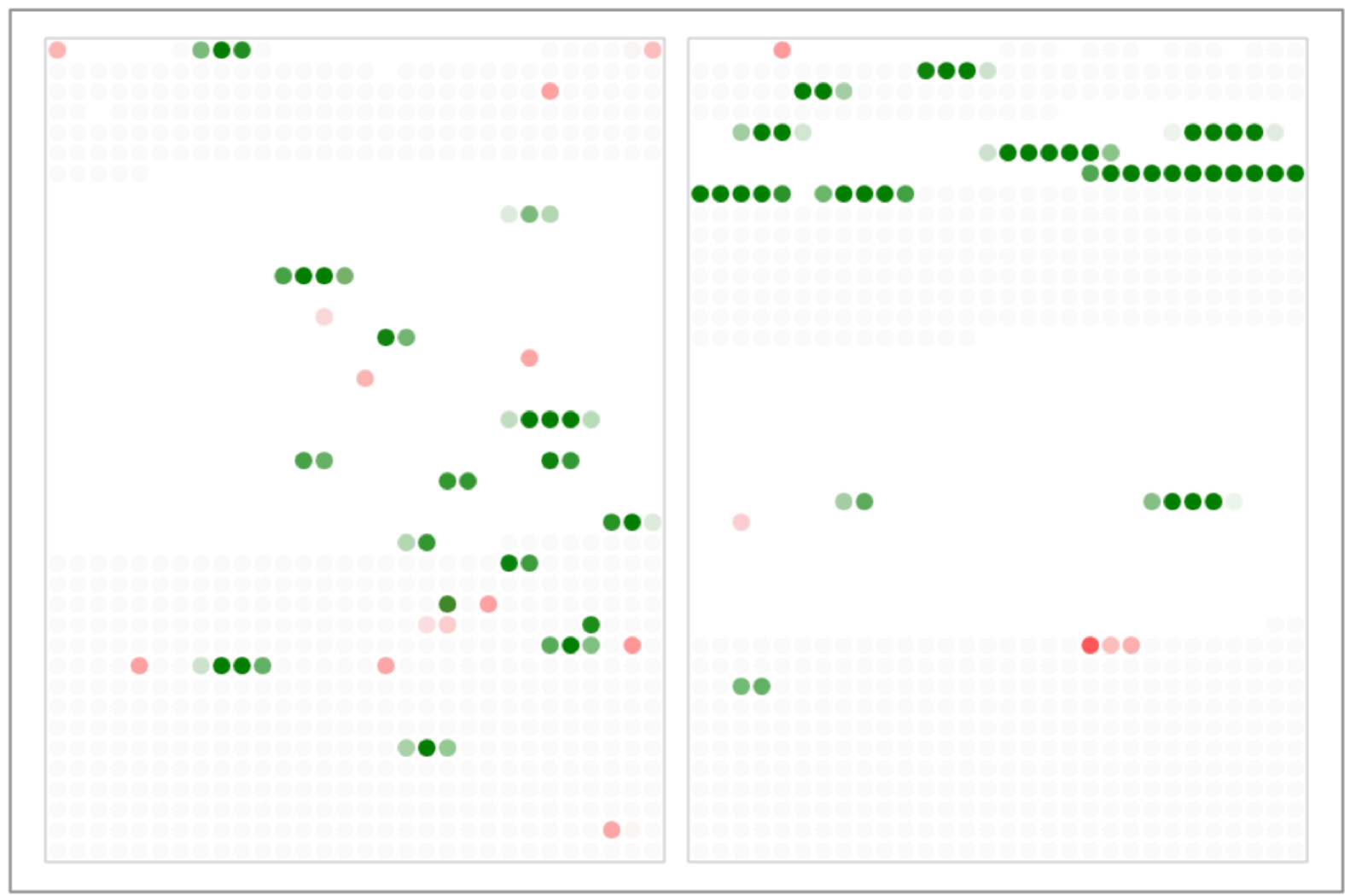

Abbildung 26: Beispiele Dokumentenporträts (links: Josephson 2010, rechts: Bayerl 2009)

Im Folgenden werden weitere, ergänzende qualitative Anmerkungen zu einzelnen Texten unter verschiedenen Stichworten zusammengefasst ${ }^{294}$. Hierbei sollen Besonderheiten dokumentiert werden, einige Auffälligkeiten zum anthropozentrischen Ansatz belegt werden und an verschiedenen Beispielen aufgezeigt werden, dass der jeweils behandelte Umweltschaden nicht eindeutig oder zu gering beschrieben wird.

\section{Ungewöhnliche Schadensinterpretation:}

Shulmann_2010: Der aus menschlicher Sicht gesehene Umweltschaden hat Vorteile für die Natur, die sich dadurch selbstständig renaturieren konnte (Abs. 104).

294 Genannt sind Autor und Publikationsjahr (vgl. Anhang B für den vollständigen Quellenbeleg). Die ggfs. aufgeführten Absatzangaben beziehen sich auf die Absatzkennzeichnung der Texte in der MAXQDA-Datei. 
Studnicki_2010: An diesem Text ist interessant, dass der Schaden (Abholzung im Zusammenhang mit Bergbau) nicht zwangsläufig als Schaden interpretiert wird, sondern vielmehr als Transformation von Waldlandschaft in Agrarlandschaft, die gleichwohl nachhaltig existieren kann (Absatz 35).

\section{Quelleninterpretation:}

Singh_2000: Der einzige Text, der die Schwierigkeiten der Quelleninterpretation behandelt (Absatz 34 ff.).

Besonderheiten bzgl. Ereignis und des daraus folgenden Schadens:

Slavin_2014: Achtung: Der Schaden ist gleichzeitig das Ereignis; es handelt sich bei den Schäden (s.u.) nicht um FOLGEN des Krieges, sondern diese sind gezielt herbeigeführte Bestandteile des Krieges. Feldzerstörung z. B. ist kein Nebeneffekt durchziehender Heere, sondern eine bewusste Handlung um dem Gegner zu schaden (vgl. Abs. 138). Daraus folgt, dass die im Text beschriebenen Taten (=Schäden) nicht als wertgeladene Passagen gekennzeichnet werden; die Frage, die zu den (impliziten) Werten führt, lautet schließlich: Warum ist der Schaden unerwünscht? Somit wird hier nach den Folgen der Schäden gesucht. In der Übersicht wird trotzdem „Krieg“ als das Ereignis bezeichnet, da es sämtliche Ereignisausprägungen zusammenfasst.

Worster_1988: Etwa die erste Hälfte des Textes widmet sich der Dürre und den Folgen, also dem hier zugeordneten Ereignis bzw. Schaden. Dann holt der Autor weiter aus, um die Vorgeschichte zu erklären, wie es dazu kommen konnte, dass diese Dürre derart schwerwiegende Folgen hatte. Interessant, aber nicht relevant für den hier zu beurteilenden Schaden... (Die Entfernung der Grassoden für den Weizenanbau könnte ebenfalls als Ereignis definiert werden, das zu den Schäden führte. Damit wären es aber zwei Ereignisse, was zu einem Auswertungs-/Vergleichbarkeitsproblem zwischen den Texten führen würde. Dies ist jedoch eigentlich kein Problem, da der letztendliche Schaden (Staubstürme bzw. ihre Folgen) derselbe ist und für die Analyse des Textes primär der Schaden relevant ist. Somit wird als Ereignis die Dürre betrachtet.)

Anthropozentrischer Ansatz:

Bayerl_1989: Ein im Text behandelter alter Text erwähnt als Umweltschaden die Ausrottung verschiedener Tiere, allerdings nur, um im nächsten Satz darauf hinzuweisen, dass diese als Nahrung des Menschen dienten (Absatz 252). Es folgen weitere Aussagen, die deutlich machen, dass ein Umweltschaden für den Autor (des alten Textes) eine Einschränkung oder einen Verlust des Nutzens der Natur für den Menschen voraussetzt. 
Bankoff_2007: Es geht u. a. um durch Überflutungen getötete Haus-/Nutztiere. Wie die Zusammenfassung ankündigt, geht es um die Anzahl getöteter Tiere und die Auswirkungen auf den Menschen. Der Tod der Tiere ist schlecht, da:

- Verlust von Arbeitstieren > Ernteausfälle > weniger Nahrung für den Menschen > Gesundheitsgefahr für den Menschen (Absatz 17),

- Verlust von Lastentieren > mehr Arbeitslast für den Menschen (Absatz 17),

- Kadaver Seuchen verbreiten > Gesundheitsgefahr für den Menschen (Absatz 51).

Mann_1995: Der Autor lehnt den anthropozentrischen Ansatz ab (Absatz 20, letzter Satz). Dies ist insofern interessant, da sich 22 von 27 Wertefundstellen in diesem Dokument auf anthropozentrische Werte beziehen.

Rohr_2003: Dieser Aufsatz behandelt u. a. die Wahrnehmung von außergewöhnlichen Naturereignissen als Katastrophe. Der folgende Satz ist deshalb interessant, da er impliziert, dass, sobald Menschen bei einem solchen Ereignis zu Schaden kommen, dies automatisch eine Katastrophe darstellt. "On the other hand, an abnormal natural event was not automatically a disaster, if people were not hurt. So, only the perception and interpretation of man is responsible for ,creating' a disaster.“ (Abs. 27) Dies sagt einiges über die Hierarchie der Werte bezüglich Natur/Umwelt und Mensch aus. Ein nicht-anthropozentrischer Ansatz dürfte ein Ereignis mit wenigen menschlichen Opfern kaum als Katastrophe werten, insofern die anderen Auswirkungen nicht sonderlich dramatisch oder weitreichend sind. Z. B. wäre eine Lawine, die einen Skifahrer tötet, nach o.g. Ansatz eine Katastrophe. Eine Lawine hingegen, die keine Menschen tötet, aber einen Teil des Lebensraumes einer Tierart zerstört, wäre demnach keine Katastrophe (zumindest nicht automatisch).

Swanson_2012: Insbesondere das 2. Beispiel (ab ca. Absatz 175) zeigt, dass die Diskussion um „wilderness“ keineswegs einfach ist. Was ist denn die „unberührte“ Natur, die es zu bewahren gilt? Und wenn der Mensch „beschützend“ eingreift, um einen bestimmten Zustand zu konservieren, ist es eine paradoxe Situation: Um die Natürlichkeit der Natur zu erhalten, greift der Mensch künstlich ein, indem er natürliche Vorgänge bzw. Veränderungen steuert oder unterbindet. Der Autor stellt wiederholt die Behauptung auf, dass alle Bemühungen zum Naturschutz auf anthropozentrischen Bedürfnissen beruhen: „(...) we must understand that our decisions to preserve or manage all landscapes are rooted in our own very human needs and desires. (...) managers must recognise that their preservation efforts place an anthropocentric value on nature." (Absatz 267)

Umweltschaden nicht eindeutig oder zu gering beschrieben:

McClenachan_2013: Bemerkenswert ist, dass der Text nur bis zur Stufe „weniger Fisch“ vordringt bzw. selten noch eine Verschlechterung des Ökosystems anführt. Die Folgen der 
Überfischung werden nicht weiter erörtert. Andererseits ist anzumerken, dass das Thema pragmatisch analysiert wird, d. h. wertende Äußerungen sind selten.

Romero_2002: Es werden ausschließlich Reduktion von Artenbeständen und Ausrottung von Arten als Folgen der Jagd genannt, ohne dies zu konkretisieren. Nur an einer Stelle wird Whale watching erwähnt (das mangels Walen nicht mehr möglich ist).

Cyffka_2007: Der Begriff „Schaden“ wird vielfach verwendet, allerdings ohne konkret zu beschreiben, worin dieser eigentlich besteht (insbesondere Absatz 108-122). So auch beim beschriebenen „Landschaftsverbrauch“: Dieser scheint etwas „Negatives" im Sinne eines Schadens zu sein, worin dieser besteht, bleibt allerdings unklar.

Amate_2013: Es wird im Text sehr oft von "environmental problem“ und "soil loss“ gesprochen, aber der Autor erklärt nicht, worin das Problem bzw. das Problem des Bodenverlustes eigentlich besteht. ${ }^{295}$

Denning_2014: Hier zeigt sich, dass „Umweltschaden“ als Begriff oftmals nicht eindeutig ist. Während die Veränderung bzw. Anpassung der alpinen Landschaft an die Bedürfnisse der Skifahrer von Naturliebhabern als Schaden betrachtet wird (Zerstörung der unberührten Natur, Beeinträchtigung der Schönheit der Natur durch Pisten, Hotels, Lifte etc.), interpretieren andere diese Entwicklung als Fortschritt, als Kultivierung oder sogar Verbesserung der ursprünglichen Landschaft (vgl. Absatz 133 ff.). Ästhetische Aspekte sind demnach schwieriger zu bewerten als z. B. Erosion infolge der Veränderung; hier dürfte man sich einig sein, dass Erosion einen Schaden darstellt. Interessanterweise behandelt der Aufsatz (fast) ausschließlich die ästhetischen Aspekte der Veränderung im Zusammenhang mit der ökonomischen Entwicklung der Skitourismusindustrie. An keiner Stelle werden die Folgen der Landschaftsumgestaltung durch die Entwaldung (und daraus folgend Erosion und Hangrutsche) für das Ökosystem bzw. umgebende Flora und Fauna und für den Menschen behandelt.

Mathews_2003: Hier wird oft von „environmental degradation“ oder „forest degradation“ gesprochen und es klingt, als ob dies etwas Schlechtes wäre. Aber es wird kaum tatsächlich als schlecht benannt oder beschrieben, warum diese „degradation“ schlecht ist (z. B. Absatz 170).

\footnotetext{
${ }^{295}$ Wenn die Folgen bzw. das Problem eindeutig sind, erübrigen sich solche Ausführungen natürlich. In diesem Fall sind aber durchaus verschiedene Probleme denkbar wie z.B. geringere Bodenfruchtbarkeit und daraus folgend weniger Nahrungsmittel, Verwüstung von Landstrichen da Pflanzen im Boden keinen Halt mehr finden und daraus folgend eine Unbewohnbarkeit für den Menschen oder geringere Wasserspeicherfähigkeit des Bodens.
} 


\subsection{Auswertung nach Wertearten}

Zur Auswertung werden die einzelnen Werte verschiedenen Arten zugeordnet. ${ }^{296}$ Die Wertearten „anthropozentrisch“297 und „nicht-anthropozentrisch“ sind bereits im Wertekategoriensystem präsent. Über alle Texte zeigt sich ein Verhältnis von $82 \%$ anthropozentrischen Werten zu 18\% nicht-anthropozentrischen, gemessen an der Anzahl der Fundstellen. Eine weitere Paarung von Wertearten ist die Aufteilung nach materiellen und immateriellen ${ }^{298}$ Werten. Hier weisen die Fundstellen einen Anteil von $51 \%$ materiellen und 30\% immateriellen Werten auf. Des Weiteren wird unterschieden nach Existenzialwerten und Nicht-Existenzialwerten. Existenzialwerte sind "Lebensgrundlage" und „Gesundheit". 299 Das Verhältnis von Existenzialwerten zu Nicht-Existenzialwerten liegt bei $39 \%$ zu $61 \%$. Insgesamt enthält das Kategoriensystem 20 Nicht-Existenzialwerte.

Bei der Paarung materiell und immateriell wurde die Wertekategorie "Gesundheit" ausgespart. ${ }^{300}$ Zwar ist Gesundheit aufgrund der physischen Merkmale kein immaterieller Wert, allerdings kann Gesundheit auch nicht den materiellen Werten zugeordnet werden, da sie weder getauscht noch verkauft werden kann. Weiterhin ist bei den o.g. Wertearten zu beachten, dass es Überschneidungen gibt, d. h. dass jeder einzelne Wert zu drei Gruppen gehört bzw. im Fall von „Gesundheit“ zu zwei Gruppen. Abbildung 27 zeigt die Analyse der Wertearten nach Anteil Codierungen über alle Dokumente und zusätzlich nach Ländern ${ }^{301}$. Demnach sind $82 \%$ aller Codierungen der Kategorie „anthropozentrisch“ zuzuordnen. Hier gibt es zudem kaum Abweichungen in den Länderauswertungen. Als Gegenpol weist die Kategorie „nicht-anthropozentrisch“ einen Anteil von nur $18 \%$ auf, wobei auch hier kaum Abweichungen in den Länderauswertungen festzustellen sind. Bei den „Existenzialwerten“ gibt es Abweichungen in beide Richtungen. Während Texte aus den USA mit einem Anteil von $41 \%$ fast identisch mit dem Gesamtanteil (39\%) sind, unterscheiden sich die Anteile bei Texten aus Deutschland (48\%) und dem UK (32\%) um 16 Prozentpunkte. Bei der entgegengesetzten Werteart „Nicht-Existenzialwerte“ gibt es die gleichen Abweichungen - in die andere Richtung - vom Gesamtanteil, der bei $61 \%$ liegt. Insgesamt enthält das

\footnotetext{
${ }^{296}$ Vgl. Abbildung 27, S. 97.

${ }^{297}$ Vgl. Kapitel 2.2.5.

298 Die Kategorie „Gesundheit“ wird weder zur materiellen noch zur immateriellen Werteart gezählt. Der Codierungsanteil von "Gesundheit" liegt bei $19 \%$ und ergibt zusammen mit den Wertearten "materiell“ und „,immateriell“ $100 \%$. Vgl. hierzu auch Fußnote 212, S. 47. Materielle Werte sind IS, ÖK, GE, ZH, RL, EQ, LG. Alle anderen bis auf GE sind immaterielle Werte. Siehe auch Anhang D Wertekategoriegruppen - Zuordnung der Werte.

${ }^{299}$ Vgl. Fußnote Nr. 169, S. 37.

300 Das bedeutet für die Interpretation von Abbildung 27, dass die Summe der Arten „materiell“ und „immateriell“ erst zusammen mit dem Anteil der Kategorie „Gesundheit“ (19\%) 100\% ergeben.

301 „Nach Anteil Codierungen“ bedeutet, dass z.B. die Anzahl der Wertefundstellen der materiellen Werte einen Anteil von $51 \%$ an der Gesamtanzahl aller Wertefundstellen hat. Bezogen auf die einzelnen Länder bedeutet es, dass z. B. die Anzahl der Wertefundstellen der materiellen Werte in Texten aus Deutschland einen Anteil von $58 \%$ an der Gesamtanzahl aller Wertefundstellen in Texten aus Deutschland hat.
} 
Kategoriensystem 20 Nicht-Existenzialwerte. Die dritte Paarung besteht aus der Werteart „materiell“ mit einem Gesamtanteil von 51\% und der Werteart „immateriell“ mit einem Gesamtanteil von 30\%. In der Werteart „materiell“ zeigen Texte aus den USA mit $41 \%$ einen etwas unterdurchschnittlichen Anteil und Texte aus Deutschland einen etwas höheren Anteil von $58 \%$. Bei der Werteart „immateriell“ gibt es ebenfalls leichte Abweichungen, und zwar hier bei Texten aus Deutschland (23\%) und Texten aus dem UK (35\%).

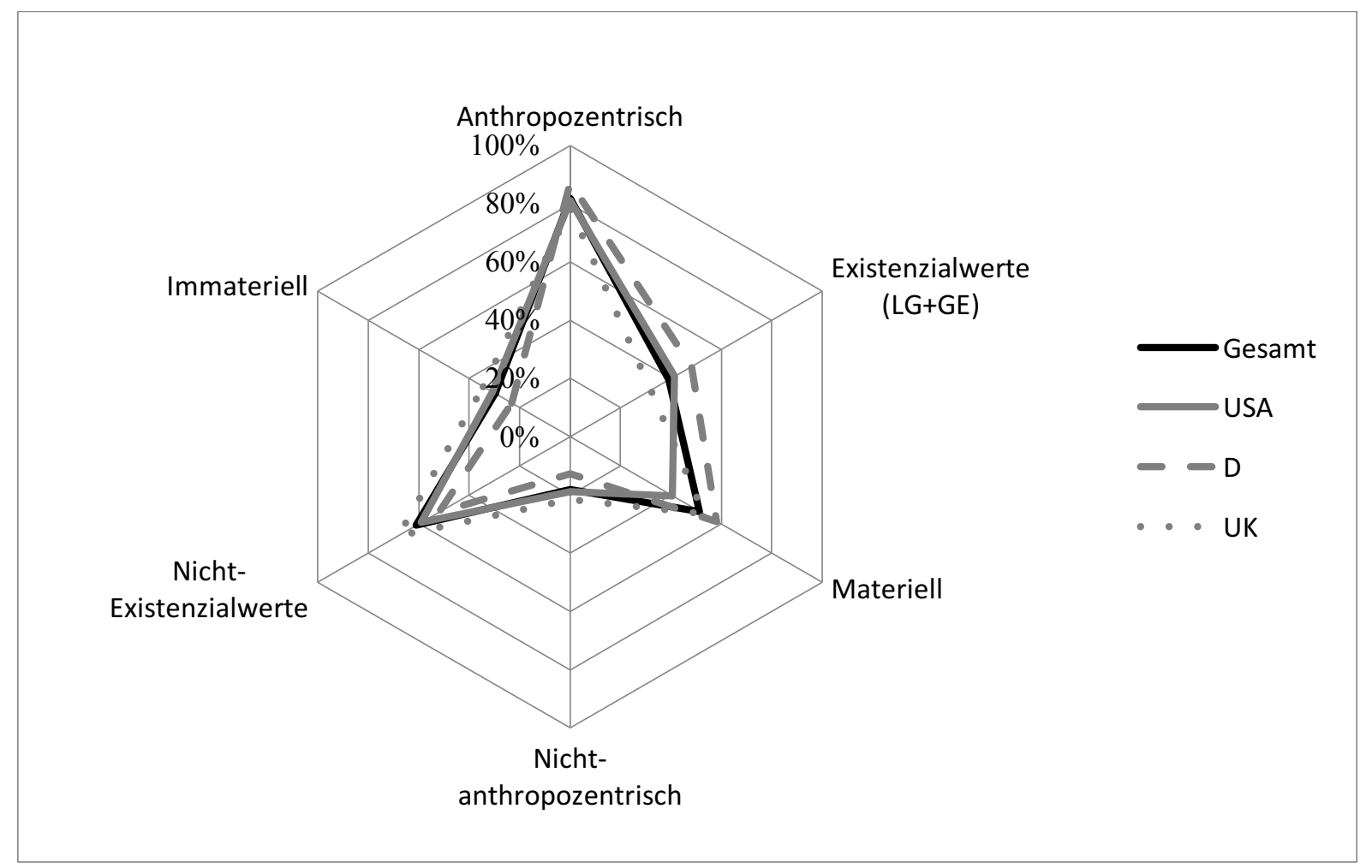

\section{Abbildung 27: Wertearten (nach Anteil Codierungen)}

Bei der Auswertung der Wertearten nach Anteil Codierungen lässt sich insgesamt feststellen, dass anthropozentrische Werte mit $82 \%$ eine deutliche Mehrheit bilden. Auch die Werteart der Nicht-Existenzialwerte überwiegt klar mit $61 \%$, ebenso die der materiellen Werte mit $51 \%$. Des Weiteren sind nur kleinere Abweichungen zwischen den Ländern zu erkennen.

In Abbildung 28 ist die Analyse der Wertearten nach Anteil Dokumente ${ }^{302}$ dargestellt. Die Art der anthropozentrischen Werte ist mit einem Gesamtanteil von 98\% in fast allen Dokumenten vertreten. Texte aus den USA weichen mit einem Anteil von 93\% leicht nach unten ab, während alle Texte aus Deutschland und aus dem UK anthropozentrische Werte enthalten. Auf der anderen Seite erzielen die nicht-anthropozentrischen Werte einen Gesamtanteil von 59\%. Texte aus den USA weichen kaum (57\%) davon ab, Texte aus

\footnotetext{
302 „Nach Anteil Dokumente“ bedeutet, dass die Anzahl der Texte, in denen der betrachtete Wert vorkommt einen bestimmten Anteil an der Gesamtanzahl aller Texte hat. Bezogen auf die einzelnen Länder bedeutet es, dass die Anzahl der Texte aus einem bestimmten Land, in denen der betrachtete Wert vorkommt, einen bestimmten Anteil an der Gesamtanzahl aller Texte aus diesem Land hat.
} 
Deutschland zeigen mit einem Anteil von 45\% schon eine größere Abweichung, insbesondere im Vergleich mit Texten aus dem UK, die einen Anteil von 88\% aufweisen. Die Werteart der Existenzialwerte hat einen Anteil von 89\%, von dem Texte aus den USA etwas nach unten abweichen (79\%), während Texte aus Deutschland (95\%) und dem UK (100\%) etwas nach oben abweichen. Die dem entgegengesetzten Nicht-Existenzialwerte sind dagegen in allen Dokumenten vertreten und zeigen keine Abweichungen nach Ländern. Ähnlich sieht es auch bei den materiellen Werten aus, die einen Gesamtanteil von 94\% haben, der mit einem Anteil von 100\% nur von Texten aus dem UK übertroffen wird. Die immateriellen Werte zeigen einen Anteil von $76 \%$, von dem Texte aus den USA $(71 \%)$ nur leicht nach unten abweichen und Texte aus dem UK mit einem Anteil von $88 \%$ eine Abweichung nach oben aufweisen.

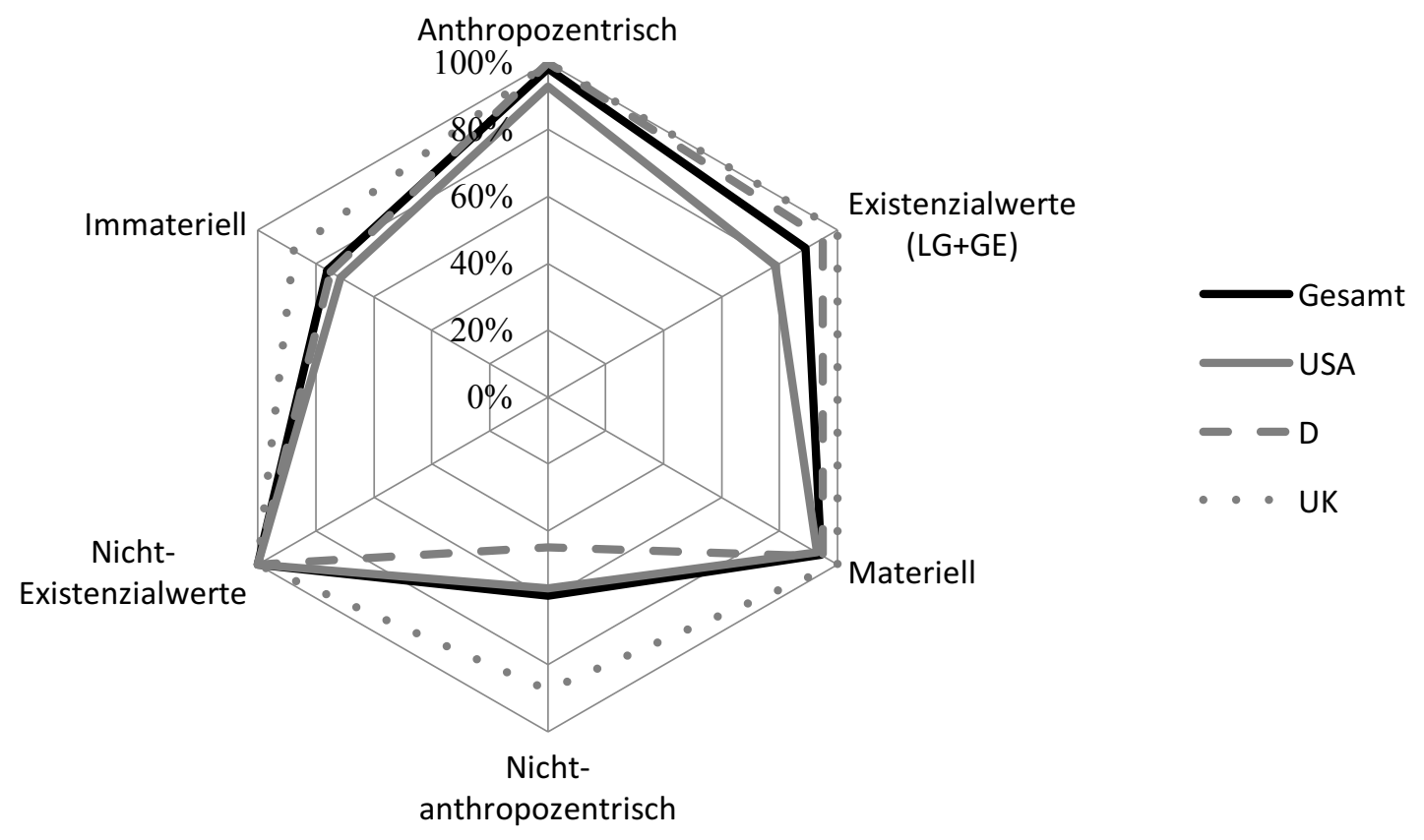

Abbildung 28: Wertearten (nach Anteil Dokumente)

Die Auswertung der Wertearten nach Anteil Dokumente zeigt insgesamt über alle Gruppen mit Anteilen von $59 \%$ bis $100 \%$, dass in den meisten Dokumenten alle Gruppen vertreten sind und sich zudem kaum länderspezifische Abweichungen zeigen. Nur bei den nichtanthropozentrischen Werten gibt es größere länderspezifische Abweichungen. Hier fallen Texte aus Deutschland auf, da sie zwar zu 100\% anthropozentrische Werte enthalten, aber nur in $45 \%$ der Texte nicht-anthropozentrische Werte zu finden sind. Weiterhin fällt auf, dass 
Texte aus dem UK bei allen Wertearten einen überdurchschnittlichen Anteil aufweisen, zum Teil bis zu $100 \%$.

In den nächsten beiden Abbildungen werden die Existenzialwerte, die die beiden übergeschichtlichen, für den Menschen existenziellen Werte ${ }^{303}$ Leben und Gesundheit enthalten, näher betrachtet. Abbildung 29 zeigt dafür die sechs Unterkategorien der Existenzialwerte nach Anzahl der Codierungen. Auf Platz 1 liegt „Nahrung“ mit 130 Fundstellen. Es folgt „Krankheit, Verletzungen“ mit 96 Treffern und auf Platz 3 „Tod“ mit 59 Treffern. „Wasser“ liegt mit 38 Fundstellen auf Rang 4, gefolgt von „Hunger" und „Luft“ mit jeweils 10 Treffern.

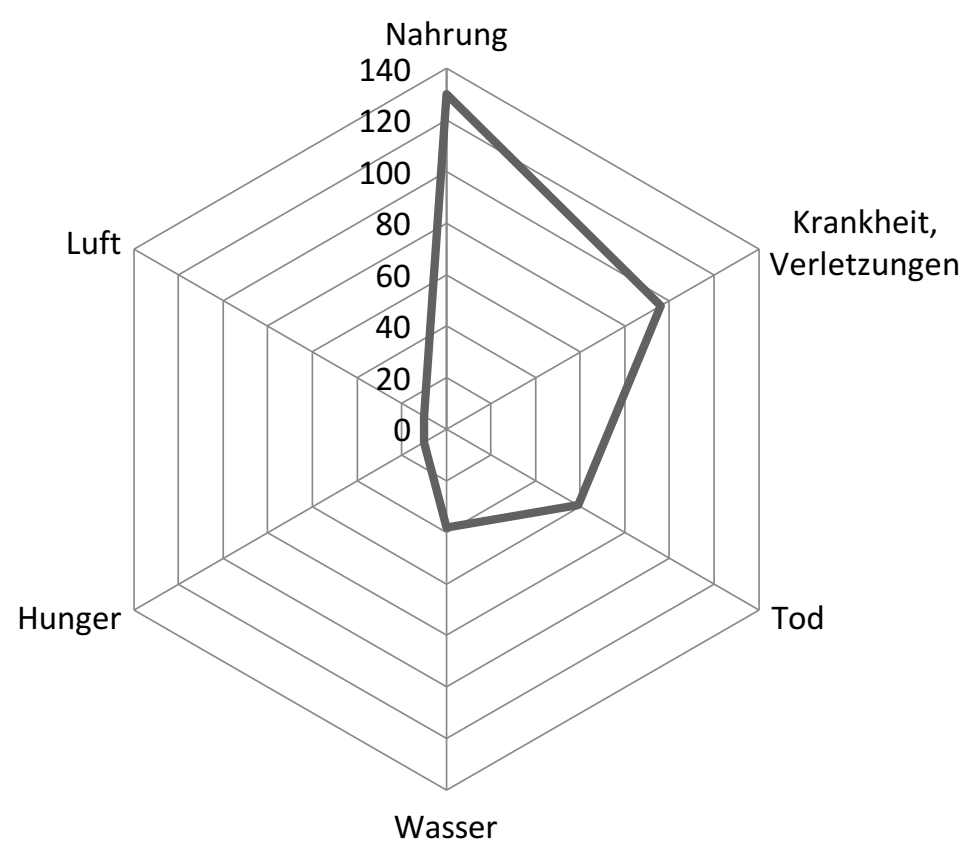

Abbildung 29: Existenzialwerte (nach absoluter Anzahl Codierungen)

Betrachtet man die Verteilung der Existenzialwerte nach Ländern, offenbaren sich einige Unterschiede. Abbildung 30 zeigt den Anteil der Dokumente, in denen der jeweilige Wert vorkommt. Hier ist die Rangfolge im Vergleich zur Auswertung nach Anzahl der Codierungen unverändert und auch hier zeigen sich große Differenzen zwischen den Ländern. Insgesamt kommt „Nahrung" in 65\% aller Dokumente vor. Bei Texten aus dem UK liegt der Anteil jedoch bei $88 \%$ während Texte aus den USA nur einen Anteil von $43 \%$ aufweisen. Texte aus Deutschland weichen mit 70\% nur leicht vom Durchschnitt ab. Bei „Krankheit, Verletzungen“ sind die Abweichung allerdings andere. Während Texte aus den USA (50\%) kaum vom Durchschnitt (54\%) abweichen, haben Texte aus Deutschland einen Anteil von 65\% und Texte aus dem UK sogar einen Anteil von 75\%. „Tod“ zeigt nur kleinere Abweichungen bei einem Gesamtanteil von 44\%, während „Wasser“ wieder große länderspezifische

\footnotetext{
${ }^{303}$ Vgl. dazu die Ausführungen von Stern bzw. Fußnote 170, S. 37.
} 
Differenzen aufweist. Texte aus dem UK liegen mit einem Anteil von 13\% deutlich unterhalb des Durchschnitts von 31\%, andererseits liegen Texte aus Deutschland mit einem Anteil von $50 \%$ klar über dem Durchschnitt. Auch die Unterschiede bei „Hunger“ und „Luft“ werden nun deutlicher. „Hunger" kommt nur in 5\% der Texte aus Deutschland vor, aber in $21 \%$ der Texte aus den USA und in 25\% der Texte aus dem UK (Durchschnitt 13\%). Da es bei „Luft“ weder in Texten aus den USA noch aus dem UK Treffer gab, liegt auch der Anteil der Dokumente bei 0\%. Dafür kommt „Luft“ in jedem vierten Text aus Deutschland vor.

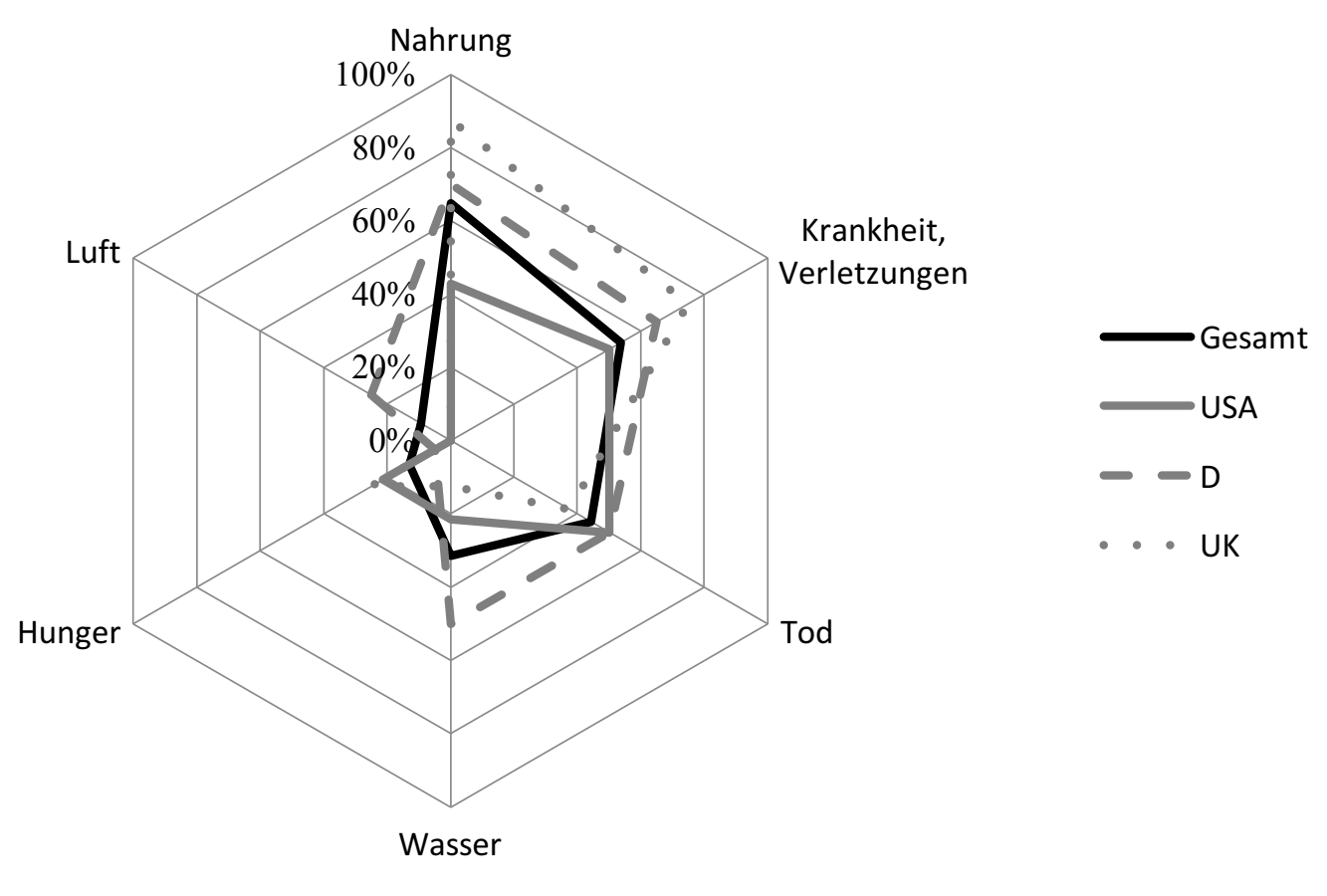

Abbildung 30: Existenzialwerte (nach Anteil Dokumente und Ländern)

Zusätzlich zu den bereits analysierten Wertekategoriengruppen werden die gewonnenen Daten nun nach den Kategorien der Ökologischen Ökonomie ${ }^{304}$ ausgewertet, da die Anteile von nutzungsabhängigen Werten (Use Values) einen Hinweis darauf liefern können, inwiefern ökonomisierbare Werte eine Rolle spielen. Dafür wurden die beiden Hauptgruppen „Use Values“ und „Non-Use Values“ verwendet, wobei die Gruppe „Use Values“ noch in „Direct Use Values“ und „Indirect Use Values“ unterteilt wurde. Abbildung 31 zeigt die Verteilung nach Anzahl der Codierungen. 74\% der Codierungen gehören demnach zur Gruppe der „Direct Use Values“, 19\% zu den „Indirect Values“ und 9\% zu den „Non-Use Values“. Daraus ergibt sich ein Verhältnis von „Use Values“ zu „Non-Use Values“ von 91\% zu $9 \%$. Länderspezifisch zeigen sich hier nur geringe Abweichungen. Der große Anteil

${ }^{304}$ Siehe Abbildung 2, S. 49 für die Kategorien der Ökologischen Ökonomie und Anhang D für die Zuordnung der Werte. 
nutzungsabhängiger Werte lässt darauf schließen, dass die Nutzbarkeit der Umwelt offenbar einen hohen Stellenwert in umweltgeschichtlichen Texten darstellt.

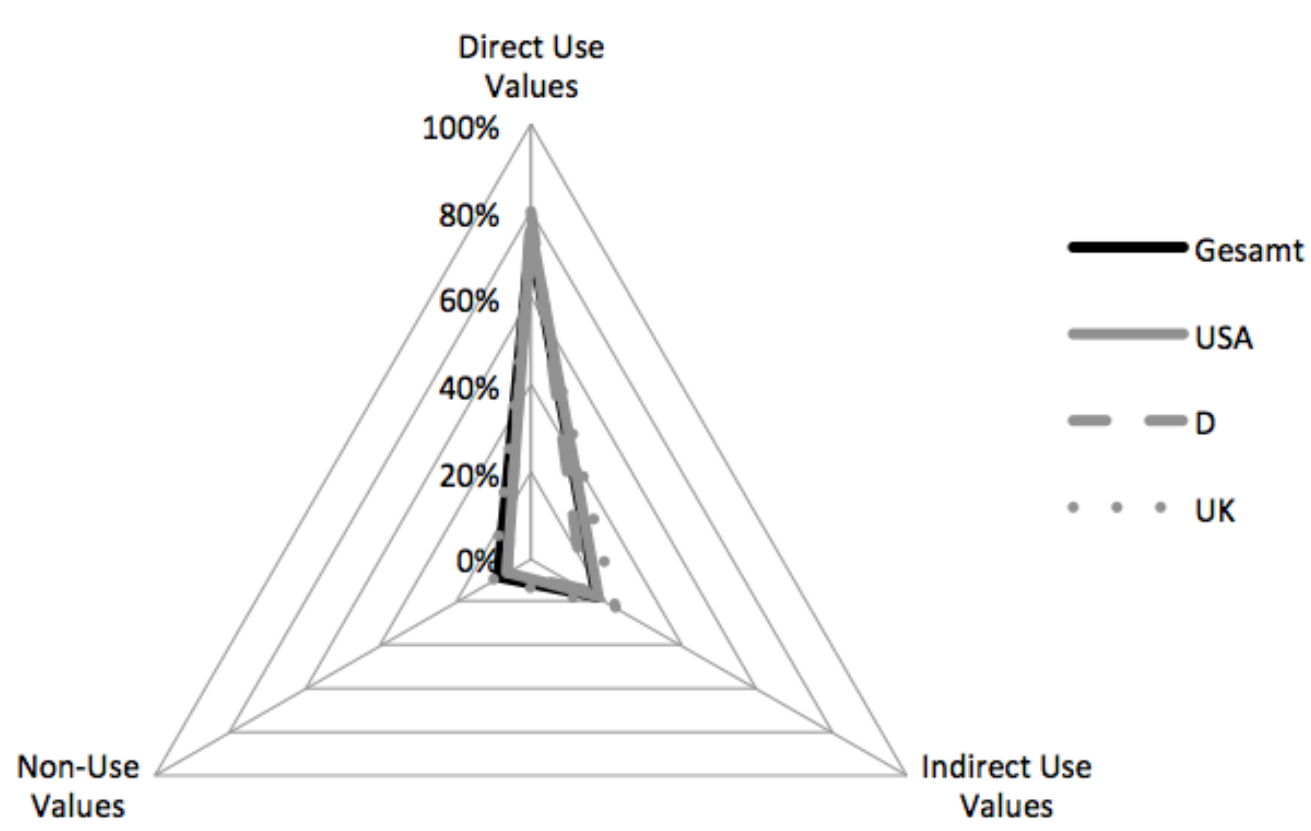

Abbildung 31: Use / Non-Use Values (nach Anteil Codierungen)

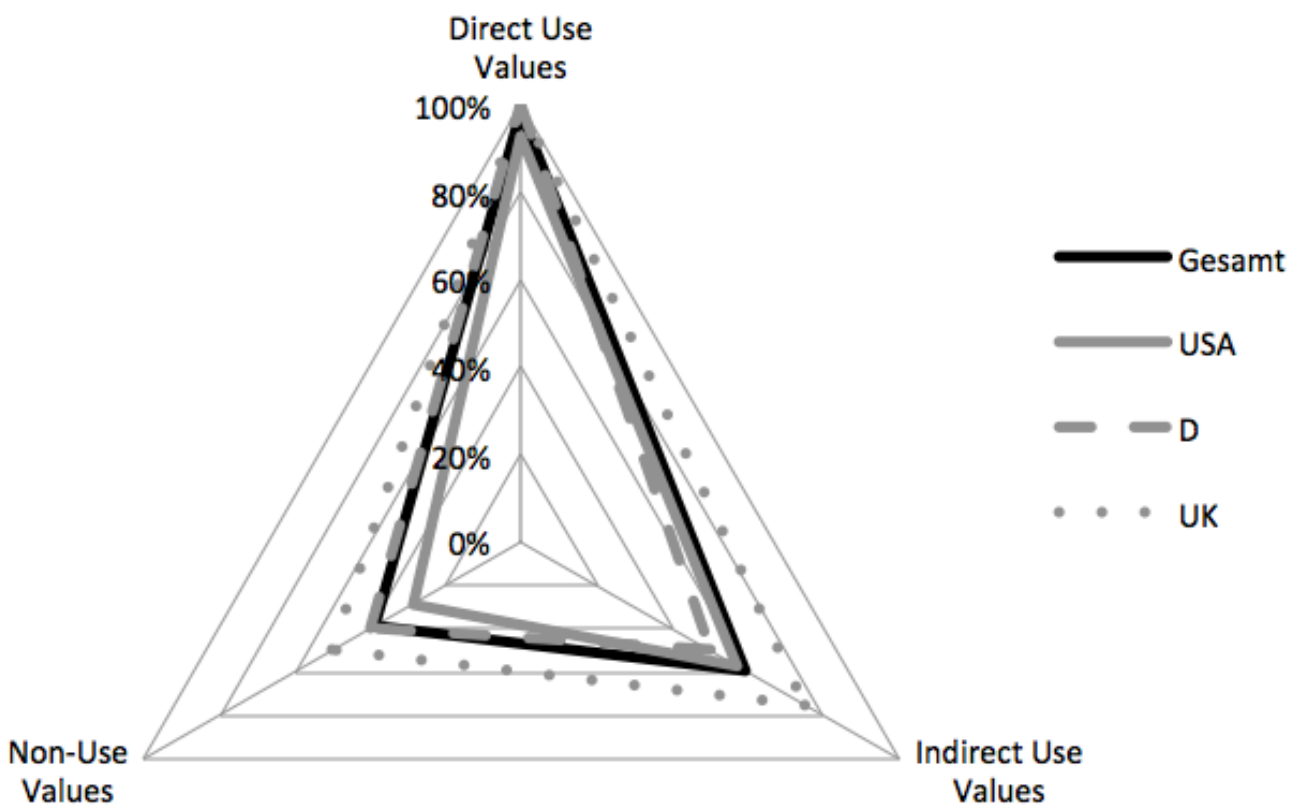

Abbildung 32: Use / Non-Use Values (nach Anteil Dokumente) 
Die Auswertung nach Anzahl der Dokumente ist in Abbildung 32 dargestellt. Es zeigt sich ein ähnliches Bild. „Direct Use Values“ weisen mit einem Anteil von 98\% die größte Verbreitung auf, in Texten aus Deutschland und dem UK liegt der Anteil sogar bei 100\%. Die Gruppe der "Indirect Use Values" hat insgesamt einen Anteil von 59\%, bei Texten aus dem UK liegt der Anteil sogar bei 75\%. Der Anteil von 39\% der "Non-Use Values“ relativiert den vergleichsweise geringen Anteil von 9\% an der Anzahl der Codierungen. Texte aus den USA (29\%) weisen eine Abweichung nach unten auf, während Texte aus dem UK (50\%) eine Abweichung nach oben zeigen. 


\section{Dominierende Werte}

\subsection{Systematik der Ermittlung}

Anhand der beiden Kriterien Anteil der Codierungen eines Wertes an der Gesamtsumme der Wertecodierungen (Anteil Codierungen) und Anteil der Dokumente in denen der Wert vorkommt (Anteil Dokumente) kann jeweils eine Rangliste nach Häufigkeit erstellt werden. Abbildung 33 zeigt die nach diesen beiden Kriterien jeweils zehn häufigsten Werte. ${ }^{305}$ Zum besseren Verständnis werden die Wertebezeichnungen „Krankheit, Verletzungen (KR)“ und „Tod (TO)“ im Folgenden nicht mehr nach dem zugrundeliegenden Schaden benannt (vgl. Abschnitt 4.2), sondern nach dem zugrundeliegenden Wert bezeichnet, also „KR > Gesundheit" und „TO > Leben“. Beim Vergleich der Ranglisten nach Anteil Codierungen und Anteil Dokumente wie in Abbildung 33 dargestellt, wird deutlich, dass die ersten fünf Plätze jeweils von denselben Werten belegt sind. Ab Platz 6 ergeben sich teilweise andere Platzierungen derselben Werte, aber es tauchen auch unterschiedliche Werte auf.

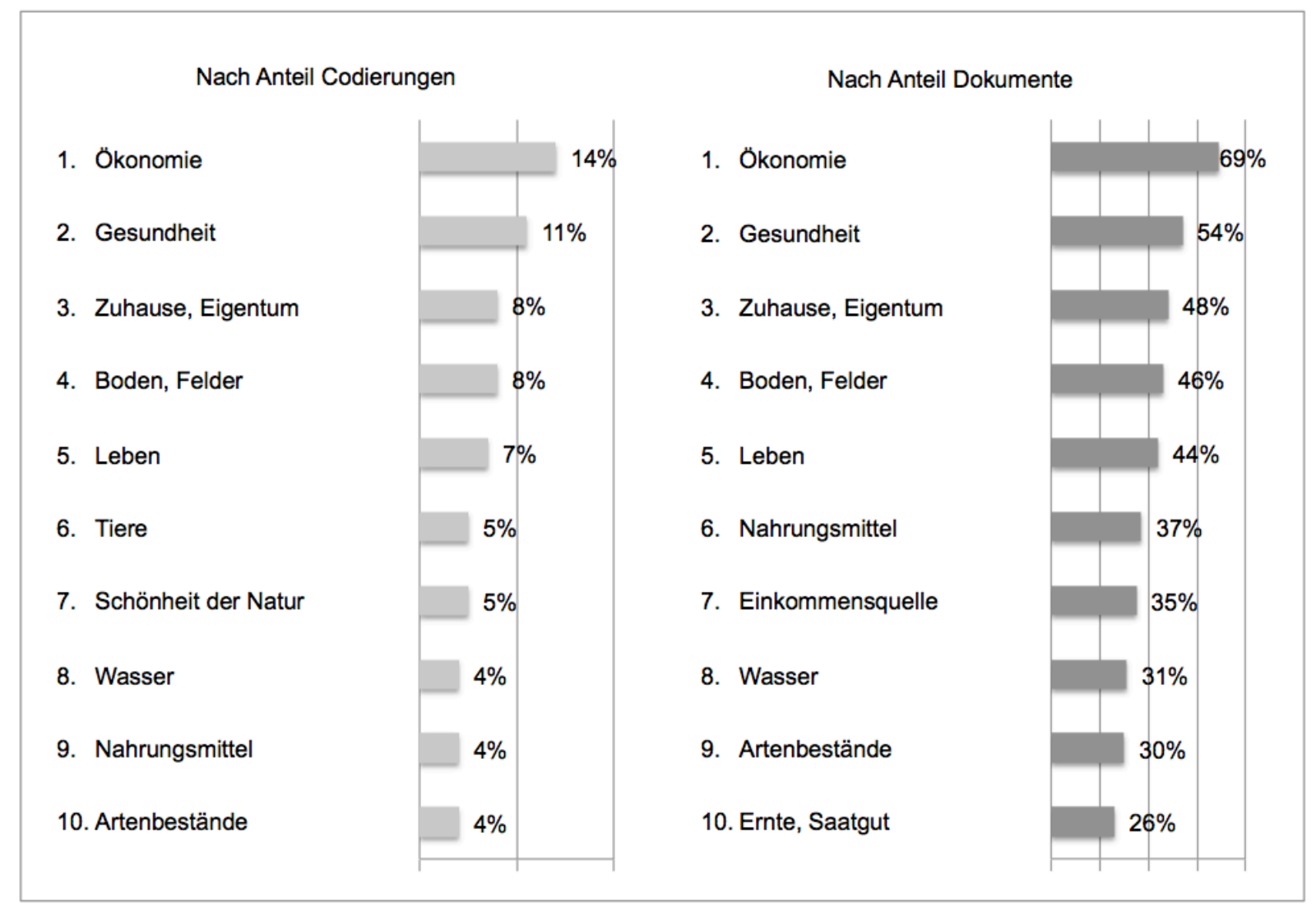

\section{Abbildung 33: Werteranglisten nach Anteil Codierungen und Anteil Dokumente}

Für die Ermittlung der dominierenden Werte ist nicht nur die Anzahl der Wertecodierungen von Interesse, sondern auch die Anzahl der Dokumente, in denen ein bestimmter Wert

\footnotetext{
305 In diesem Abschnitt geht es um die bestimmenden Werte, daher werden hier zunächst einmal die zehn häufigsten Werte dargestellt. Siehe Anhang C Ranglisten der Werte für die jeweils vollständigen Listen.
} 
vorkommt und zwar ungeachtet dessen wie oft. Der Vergleich zwischen meistgenannten Werten nach Anzahl der Codierungen und nach Anzahl der Dokumente kann helfen, Werte zu identifizieren, die zwar häufig aufgefunden wurden, aber nur in wenigen Texten vorkommen. Dies spricht eher für einen nicht so relevanten Wert in der Umweltgeschichte als Ganzes. Mehr Relevanz ist Werten beizumessen, die in vielen Dokumenten vorkommen, auch wenn diese insgesamt weniger Treffer erzielen. Denn wenngleich dieser Wert nicht vertieft bzw. intensiv behandelt wird, zeigt dies doch seine weite Verbreitung. Werte, die sowohl häufig genannt werden als auch in vielen Dokumenten vorkommen, sind als dominierende Werte anzusehen. Diesem Ermittlungsansatz folgend, wird eine gewichtete Rangliste erstellt, die die Anzahl Codierungen und die Anzahl Dokumente integriert. Aufgrund der oben ausgeführten höheren Relevanz von Nennung in vielen Dokumenten gegenüber der Anzahl der Wertecodierungen wird die Anzahl der Dokumente mit 2/3 berücksichtigt, die Anzahl der Codierungen mit 1/3. ${ }^{306}$ Als Vorauswahlkriterium wurde ein Vorkommen der Werte in mehr als 30\% aller Dokumente gesetzt, da aus o. g. Erwägungen eine geringe Verbreitung gegen einen bestimmenden Wert spricht. Im folgenden Abschnitt soll zunächst die länderspezifische Betrachtung erfolgen, bevor anschließend die Ermittlung der insgesamt vorherrschenden Werte durchgeführt wird.

\subsection{Länderspezifische Betrachtung}

Die länderspezifische Betrachtung erfolgt für die drei am häufigsten in der Stichprobe vertretenen Länder Deutschland, USA und UK. Abbildung 34 zeigt die Ermittlung der dominierenden Werte in Texten aus Deutschland. Auf der linken Seite sind die acht durch o. g. Vorauswahl selektierten Werte nach Anteil Dokumente sortiert dargestellt. Der jeweils untere Balken weist den Anteil Codierungen des Wertes aus. Auf der rechten Seite ist dann die gewichtete Rangliste abgebildet, die das Ergebnis der Auswertungen darstellt. Der Prozentsatz neben der Rangliste ist das Ergebnis der gewichteten Prozentsätze und zeigt, wie weit die Platzierungen auseinanderliegen. Die rechts unten in grauer Schrift dargestellten Werte gehören in dem jeweiligen Land nicht zu den vorherrschenden Werten, aber in einem der beiden anderen Länder. Daher ist deren Platzierung zum leichteren Vergleich mit aufgeführt. In Texten aus Deutschland ist demnach Gesundheit der am stärksten vertretene Wert. Mit einigem Abstand folgen dann die weiteren sieben als dominierend identifizierten Werte, die jeweils weniger Abstand zueinander aufweisen. Insgesamt fällt auf, dass alle acht Werte anthropozentrische sind und entweder zu den materiellen Werten zählen oder zur

\footnotetext{
${ }^{306}$ Es wurde testweise auch eine Auswertung mit der Gewichtung 60\% Anzahl Dokumente und 40\% Anzahl Wertecodierungen erstellt. Der Vergleich mit der gewählten Gewichtung 2/3 Anzahl Dokumente und 1/3 Anzahl Wertecodierungen zeigte, dass sich dadurch keine Unterschiede in der Gesamtrangfolge ergeben, aber die gewählte (stärkere) Gewichtung der Anzahl der Dokumente besser der stärkeren Bedeutung entspricht.
} 
Kategorie Gesundheit (Werte Gesundheit und Leben). Weiterhin gehören fünf Werte zu den Existenzialwerten, während die anderen drei ökonomisch geprägt sind (Zuhause, Eigentum; Ökonomie; Einkommensquelle).

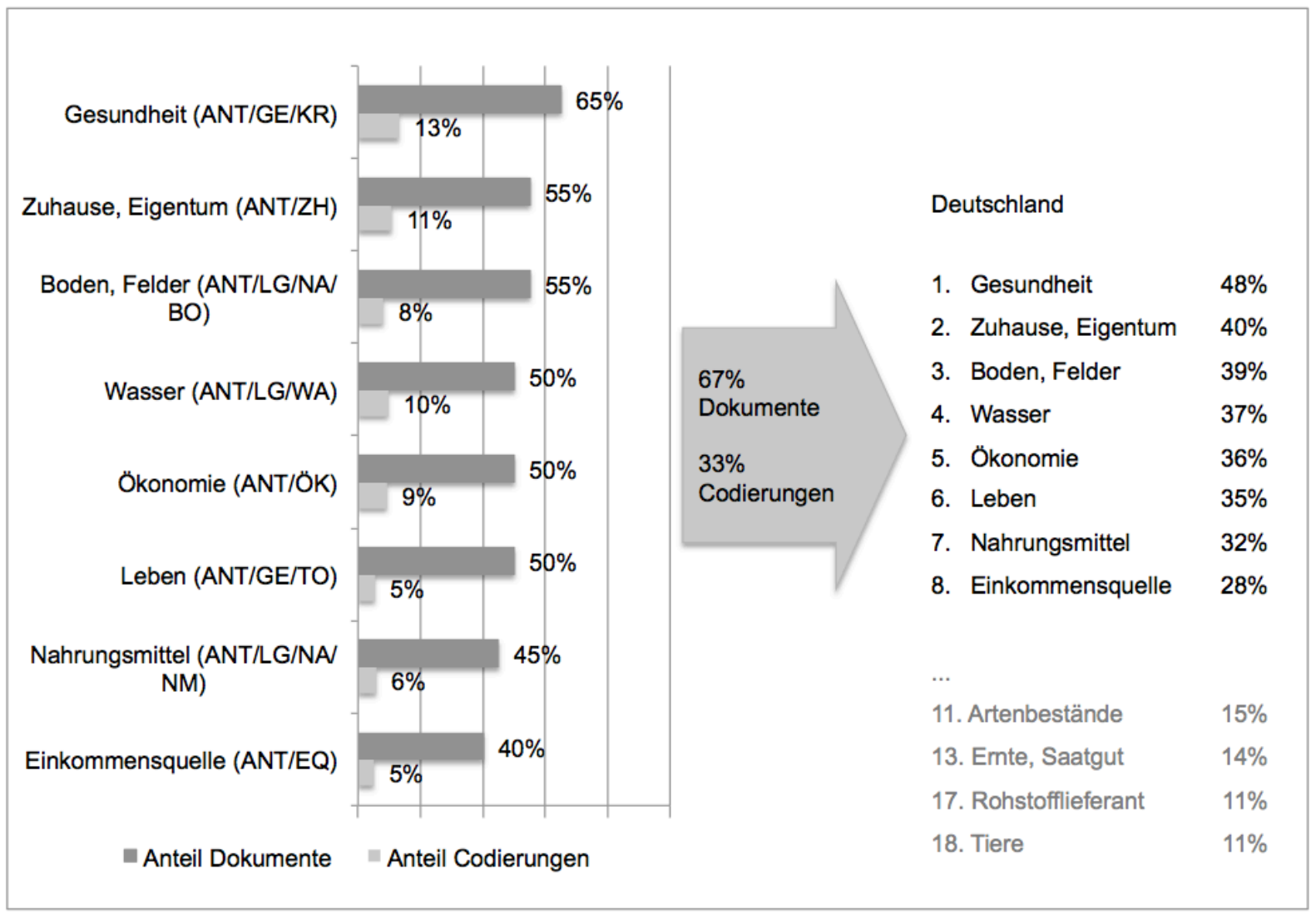

Abbildung 34: Dominierende Werte - Texte aus Deutschland

Als nächstes zeigt Abbildung 35 die Auswertung für Texte aus den USA. Hier haben nur fünf Werte einen Anteil Dokumente von mehr als 30\%. Der vorherrschende Wert ist die Ökonomie und das mit einem deutlichen Abstand zu dem zweitplatzierten Wert Gesundheit, der fast gleichrangig mit dem drittplatzierten Wert Leben ist. Mit einigen Prozentpunkten Abstand folgen dann die Werte Einkommensquelle und Zuhause, Eigentum. Auch hier sind ausschließlich anthropozentrische Werte vertreten, die entweder zu den materiellen Werten zählen oder zur Kategorie Gesundheit (Werte Gesundheit und Leben).

Die Auswertung für Texte aus dem UK ist in Abbildung 36 dargestellt. Die Vorauswahl ergibt 10 Werte. Auch hier liegt Ökonomie auf Platz 1 und zwar mit großem Abstand (19 Prozentpunkte) zum zweitplatzierten Wert Gesundheit. Insgesamt sind die Werte aber weniger ökonomisch orientiert und mit den Werten Tiere und Artenbestände erscheinen erstmals auch immaterielle Werte in den bestimmenden Werten. Dass viele verschiedene Werte in mehr als $30 \%$ der Dokumente vorkommen weist darauf hin, dass die Themen unter verschiedenen Aspekten betrachtet werden. 


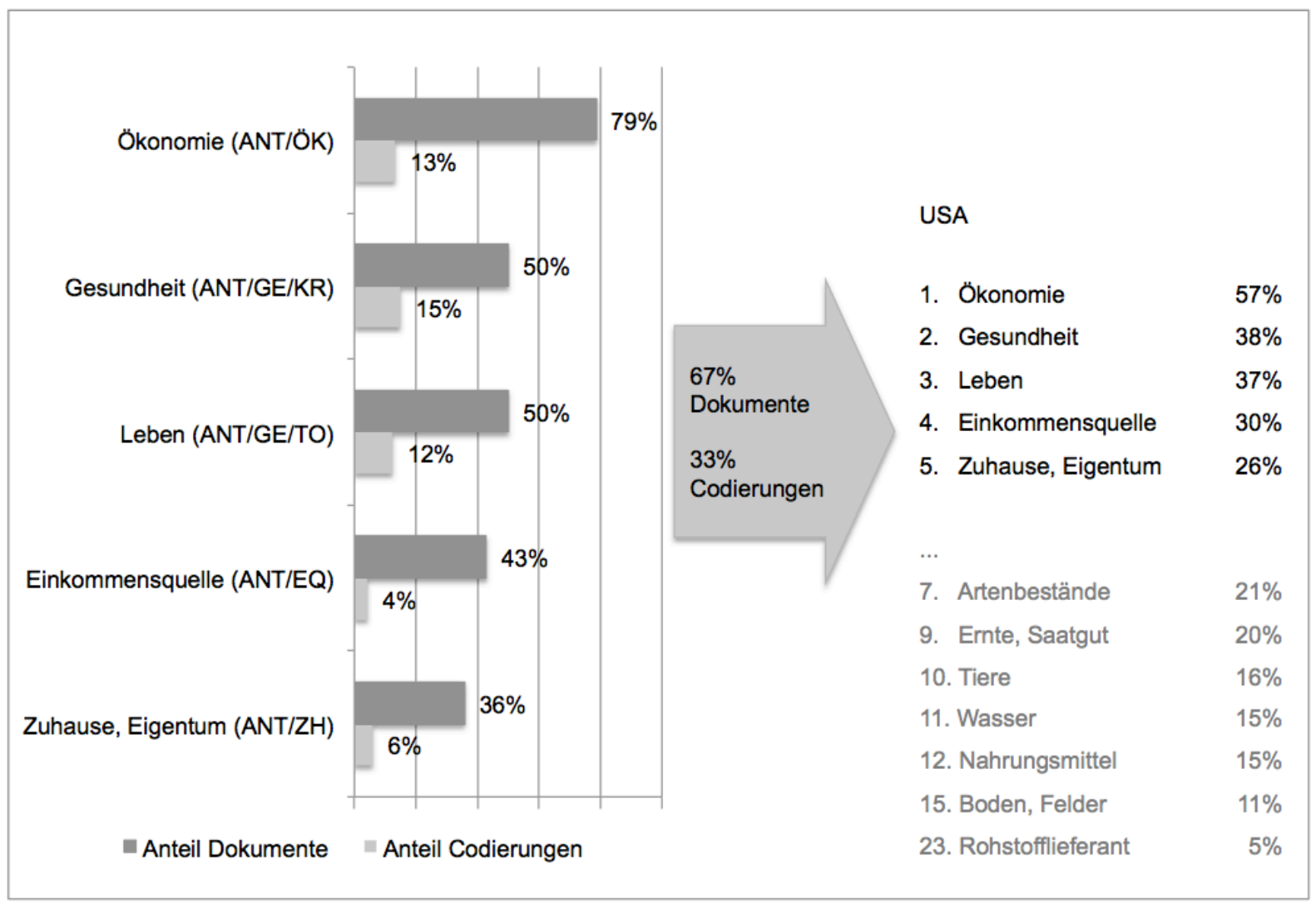

Abbildung 35: Dominierende Werte - Texte aus den USA

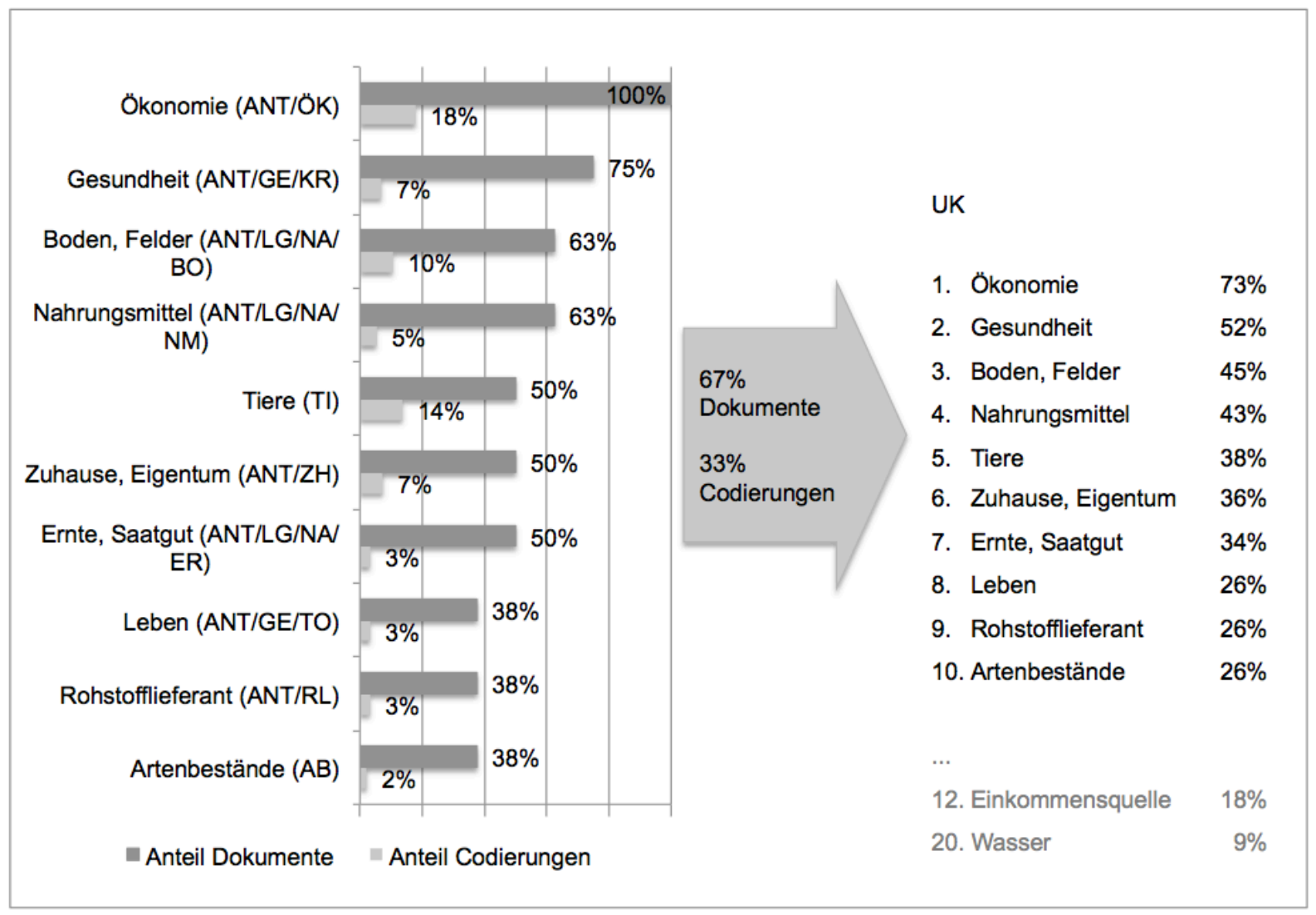

Abbildung 36: Dominierende Werte - Texte aus dem UK 
Ein Vergleich der länderspezifischen Auswertungen zeigt, dass der Wert Ökonomie in Texten aus Deutschland nur auf Platz 5 liegt, während dieser Wert sowohl in Texten aus den USA als auch aus dem UK mit deutlichem Abstand Rang 1 belegt. Der Wert Wasser der in Texten aus Deutschland den vierten Platz belegt, erzielt in Texten aus den USA nur Platz 11, in Texten aus dem UK sogar nur Platz 20. Der Wert Boden, Felder erreicht sowohl bei Texten aus Deutschland als auch bei Texten aus dem UK Platz 3, während er bei Texten aus den USA nur Rang 15 belegt. Alle fünf dominierenden Werte der Texte aus den USA sind auch in den acht Werten der Texte aus Deutschland enthalten. Der Wert Einkommensquelle liegt auf Rang 4 bei Texten aus den USA und Rang 8 bei Texten aus Deutschland, aber bei Texten aus dem UK erst auf Platz 12. Der Wert Tiere erreicht bei Texten aus dem UK Platz 5, kommt aber weder bei Texten aus Deutschland noch aus den USA in den bestimmenden Werten vor (USA: Rang 10, Deutschland: Rang 18). Dagegen wurden vier Werte bei allen drei Länderauswertungen als dominierend identifiziert: Ökonomie, Gesundheit, Leben und Zuhause, Eigentum.

Es gibt also einige Übereinstimmungen zwischen den drei Ländern, jedoch auch einige große Unterschiede. Da die Texte dieser drei Länder 78\% aller untersuchten Texte ausmachen, sind die Unterschiede bei der Ergebnisdiskussion zu berücksichtigen.

\subsection{Ermittlung der dominierenden Werte}

Die Ermittlung der insgesamt dominierenden Werte erfolgt nach demselben Ermittlungsansatz wie die soeben durchgeführten länderspezifischen Betrachtungen. Auch hier wurde als Vorauswahlkriterium ein Vorkommen der Werte in mehr als 30\% aller Dokumente gesetzt, da aus o.g. Erwägungen eine geringe Verbreitung gegen einen bestimmenden Wert spricht. Zudem bewirkt diese Grenze, dass neben den insgesamt fünf bestplatzierten Werten (vgl. Abbildung 33) noch drei Werte hinzukommen, die in den länderspezifischen Betrachtungen des vorangegangenen Abschnitts jeweils eine hohe Platzierung erreichten und daher in der Gesamtrangliste berücksichtigt werden sollten: Nahrungsmittel liegt in dem UK auf Rang 4, Wasser liegt in Deutschland auf Rang 3 und Einkommensquelle liegt in den USA auf Rang 5 . Abbildung 37 zeigt die so ermittelten acht vorherrschenden Werte. Auf diese acht Werte entfallen insgesamt $61 \%$ aller Wertecodierungen. 


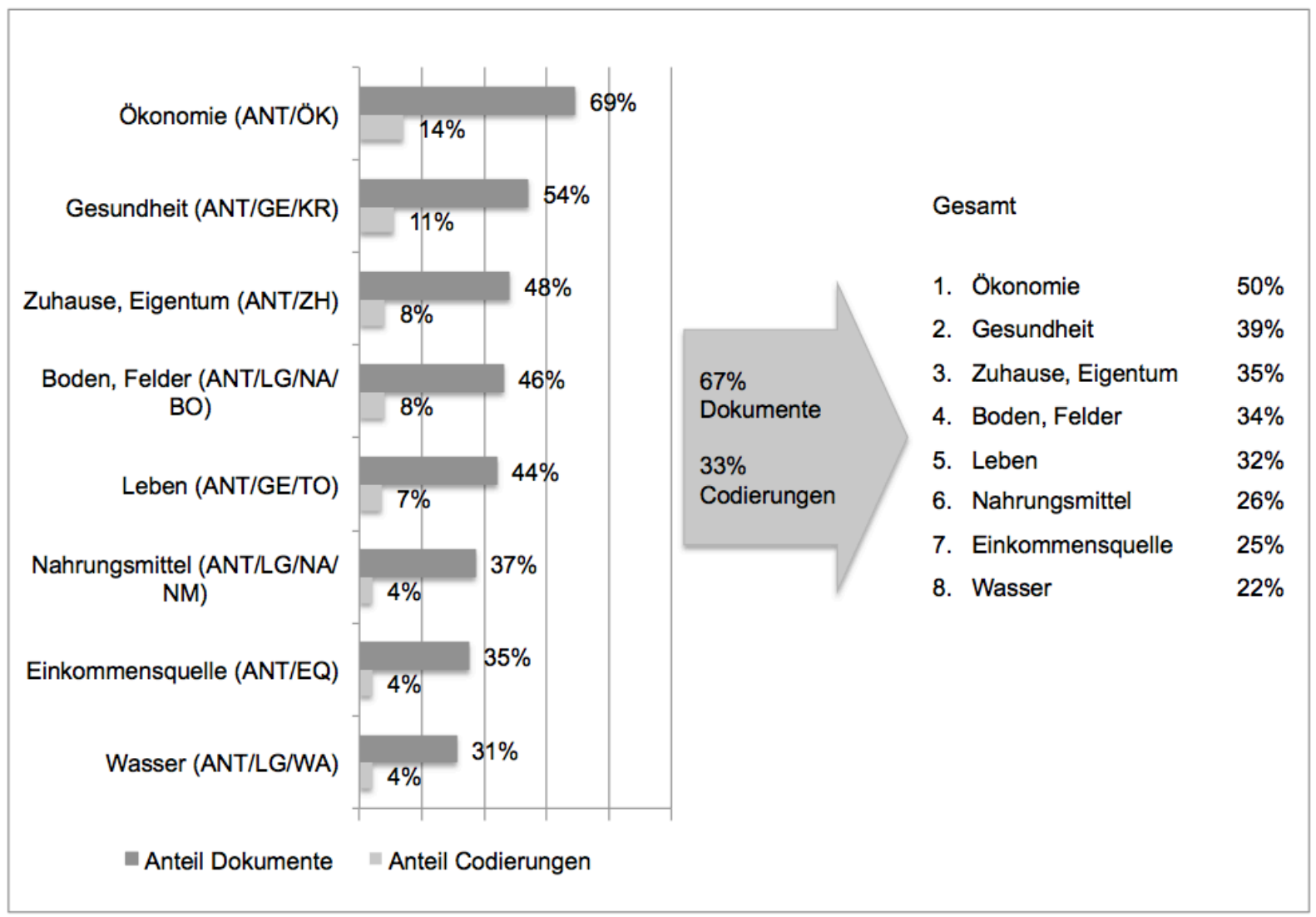

\section{Abbildung 37: Dominierende Werte insgesamt}

Zunächst ist festzustellen, dass die dominierenden Werte ausschließlich anthropozentrische Werte beinhalten (erst auf Platz 9 erscheint mit „Artenbestände“ der erste nichtanthropozentrische Wert ${ }^{307}$ ). Fünf dieser Werte gehören zur Wertekategorie Existenzialwerte, die anderen drei können unter den Bereich Ökonomie (Ökonomie; Zuhause, Eigentum; Einkommensquelle) subsumiert werden. Weiterhin sind alle acht Werte Direct Use Values. Sechs Werte sind materieller Art, während die Werte Gesundheit und Leben zur Kategorie Gesundheit gehören, die weder materiell noch immateriell ist. Ökonomie liegt mit elf Prozentpunkten Abstand auf Platz 1, die weiteren Werte folgen mit weniger Abstand. Die fünf erstplatzierten Werte liegen auch in den Auswertungen nach Anzahl Dokumente und Anzahl Codierungen ${ }^{308}$ jeweils auf den ersten fünf Plätzen.

\subsection{Einzelbetrachtung der dominierenden Werte}

In diesem Anschnitt werden die soeben identifizierten dominierenden Werte näher erörtert. Nach der Definition und Einordnung des Wertes bzgl. der Werteart und der Wertekategorie

\footnotetext{
${ }^{307}$ Vgl. Anhang C Ranglisten der Werte.

${ }^{308}$ Vgl. Abbildung 33, S. 103.
} 
wird das Werteverständnis anhand von Textausschnitten vertieft, ${ }^{309}$ bevor ein Vergleich nach Ländern erfolgt.

\subsection{1 Ökonomie}

Der Wert Ökonomie bezeichnet die wirtschaftlichen Aspekte eines Umweltschadens, d. h. die Kosten aufgrund von Umweltschäden für die Beseitigung und Lagerung, für Produktionsausfälle und nicht nutzbare Restbestände (nach Produktverbot) sowie die Kosten durch allgemeine Beeinträchtigungen des Tourismus und Preissteigerungen oder Preisverfall. Die Kosten müssen nicht quantifiziert sein. Des Weiteren ist die monetäre Bewertung eines Umweltschadens eingeschlossen, auch wenn nicht näher erläutert wird, worin der Schaden bzw. der Verlust bestehen, sowie die allgemeine Erwähnung wirtschaftlicher Schadensaspekte. Die Abgrenzung zum Wert Einkommensquelle erfolgt über die Anzahl der Produktionsstätten: Die Kosten betreffen dabei nicht nur einzelne Produktionsstätten, sondern mehrere bis hin zu ganzen Industrien.

Der Wert Ökonomie gehört zu den Direct Use Values, ist materiell und ein NichtExistenzialwert. Die folgenden Textausschnitte zeigen, dass die ökonomischen Kosten zum Teil nur angedeutet werden oder sehr allgemein formuliert sind:

- Beseitigung ausgesprochen teuer (Shulmann 2010, 113),

- economic losses (Aguirre 2012, 54),

- financial burdens (Brantz 2011, 166),

- destroying the commercial value (Crook 2002, 221),

- $\quad$ the damage ... was massive in terms of ... economic damage (de Kraker 2013, 410),

- $\quad$ economic concerns (Ross 2014, 58),

- financial consequences (Schott 2002, 165),

- decline in profits (Slavin 2014, 92),

- die finanziellen Kosten (Worster 1988, 10).

Des Weiteren werden wirtschaftliche Auswirkungen indirekt adressiert:

- $\quad$ work stoppages (Bruno 2013, 115),

- forest destruction ... wasted money by burning valuable timber (Beattie 2003, 37),

- $\quad$ oriented to the economic development (Daley 2008, 138),

- $\quad$ attempted to maintain the lucrative ski industry (Carey 2007, 123).

\footnotetext{
${ }^{309}$ Die Quellenangabe zu den Textbeispielen besteht aus dem Namen des Autors, dem Publikationsjahr sowie der Absatznummer der Fundstelle in der MAXQDA-Datei. Die vollständige Quellenangabe ist im Anhang B zu finden.
} 
Zudem fließt der ökonomische Aspekt eines Umweltschadens über die Angabe konkreter Geldbeträge bezüglich der Kosten des Umweltschadens ein:

- $\quad$ Nennung konkreter Geldbeträge (Bayerl 2009, 12; Bankoff 2007, 86; Bruno 2013, 136; Castonguay 2007, 133; Mc Farlane 2012, 68; Schott 2002, 123; Sheail 2013, 127; Smith 2012, 76; Worster 1988, 11).

Obwohl der Wert Ökonomie aufgrund seiner vergleichsweise einfach zu quantifizierbaren Natur einen hohen Anteil an konkreten Zahlen erwarten lässt, kommen 74\% der Fundstellen zum Wert Ökonomie ohne quantifizierende Attribute aus und weitere $7 \%$ beschreiben den ökonomischen Schaden nur vage (z.B. „massive“, „dramatically“, „significant“). Nur 19\% der ermittelten Textstellen enthalten konkrete Angaben zu den ökonomischen Folgen.

Abbildung 38 stellt den Wert Ökonomie im Ländervergleich dar. Es zeigen sich z.T. deutliche Unterschiede. Nach Anteil Codierungen weist der insgesamt erstplatzierte Wert Ökonomie bei den aus Deutschland stammenden Texten einen Anteil von $9 \%$ an den Codierungen auf, bei den Texten aus dem UK einen Anteil von 18\%. Dieser Unterschied findet sich ebenfalls wieder bei der Auswertung nach Anteil Dokumente. Hier liegt der Wert Ökonomie sowohl insgesamt als auch bei Texten aus den USA und dem UK auf Rang 1, bei Texten aus Deutschland jedoch nur auf Rang 4 (insgesamt Rang 5). Zudem ist hier der Anteil der Dokumente, bei denen dieser Wert vorkommt, sehr unterschiedlich: Während in $50 \%$ der Texte aus Deutschland der Wert Ökonomie auftaucht, ist dies bei allen Texten aus dem UK der Fall (100\%). Insgesamt liegt der Wert Ökonomie nur bei Texten aus Deutschland nicht auf Platz 1 sondern belegt nur den fünften Rang.

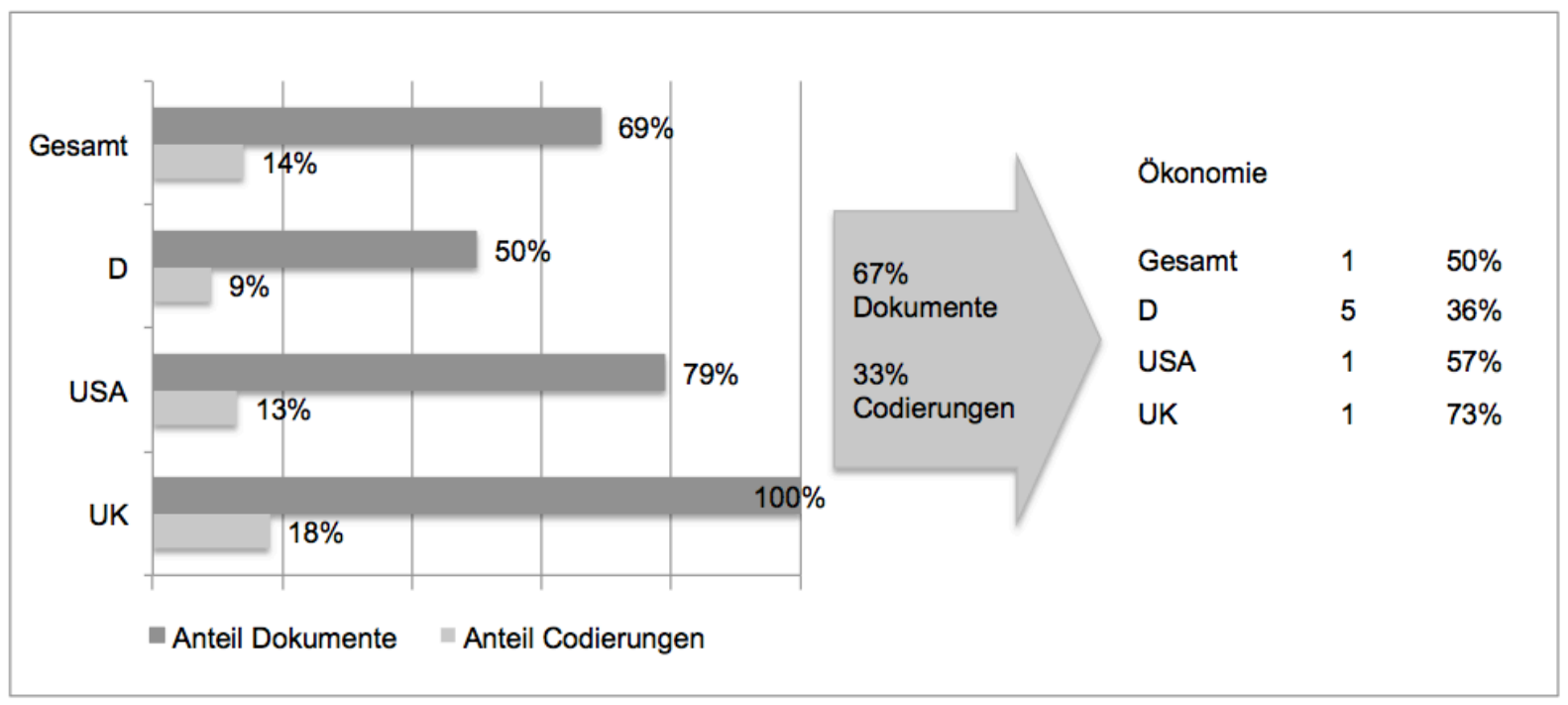

Abbildung 38: Der Wert Ökonomie im Ländervergleich 


\subsubsection{Gesundheit}

Der Wert Gesundheit beschreibt sämtliche physischen und psychischen Beeinträchtigungen (auch das Risiko einer Beeinträchtigung) der menschlichen Gesundheit ohne Todesfolge; dies schließt auch die Fortpflanzung ein.

Der Wert Gesundheit gehört zu den Direct Use Values, ist weder materiell noch immateriell und er ist ein Existenzialwert. Die folgenden Textausschnitte zeigen Formulierungen allgemeiner gesundheitlicher Bedrohungen durch den Umweltschaden, die jedoch (noch) nicht eingetreten sind:

- der menschlichen Gesundheit nachteilig (Bayerl 1989, 383),

- $\quad$ threatened the well-being ... oft he population (Castonguay 2007, 148),

- Gesundheitsgefährdung (Josephson 2010, 50),

- gesundheitsgefährdenden Chemikalien (Scheringer 2012, 54),

- $\quad$ threat to human wellbeing (Sheail 2013, 126).

Diese Beispiele beziehen sich auf festgestellte gesundheitliche Beeinträchtigungen:

- $\quad$ causing disease and unknowable damage to future generations (Buhs 2002, 75),

- $\quad$ pandemic disease (Carey 2007, 118),

- litt auch die Gesundheit des Menschen (Obertreis 2013, 24),

- their bodies suffer the consequences (Pritchard 2012, 56),

- gesundheitliche Folgen (Shulmann 2010, 9),

- $\quad$ effect on public health (Mc Farlane 2012, 53),

- $\quad$ für den Rest des Lebens Schäden davontrugen (Worster 1988, 49),

- Gesundheitsschäden (Schulze 2013, 27),

- gesundheitliche Schäden (Völkel 2014, 52),

- $\quad$ injured or injuries (Aguirre 2012, 54; Bruno 2013, 21; Schott 2002, 22).

Weiterhin zeigt sich der Wert Gesundheit über die Benennung aufgetretener Krankheiten:

- $\quad$ Konkrete Nennung von Krankheiten (Bankoff 2007, 51; Bayerl 2009, 23; Buhs 2002, 72; Mann 1995, 120; Obertreis 2013, 24; Shulmann 2010, 122; Scheringer 2012, 42; Smith 2012, 253; Worster 1988, 43).

Der Wert Gesundheit lässt sich anhand von z.B. Krankheitsraten, Anzahl erkrankter Personen oder Spezifizierung der gesundheitlichen Schäden quantifizieren. Allerdings weisen $88 \%$ der Fundstellen zum Wert Gesundheit keine quantifizierenden Attribute auf. Dagegen enthalten nur 10\% der ermittelten Textstellen konkrete Angaben und weitere $2 \%$ beschreiben den gesundheitlichen Schaden nur vage („very few cases“, „several hundred“). Damit ist Gesundheit der am seltensten quantifizierte Wert unter den dominierenden Werten (Es folgen Nahrungsmittel mit $87 \%$ und Boden mit $85 \%$ nicht quantifizierten Textstellen). 


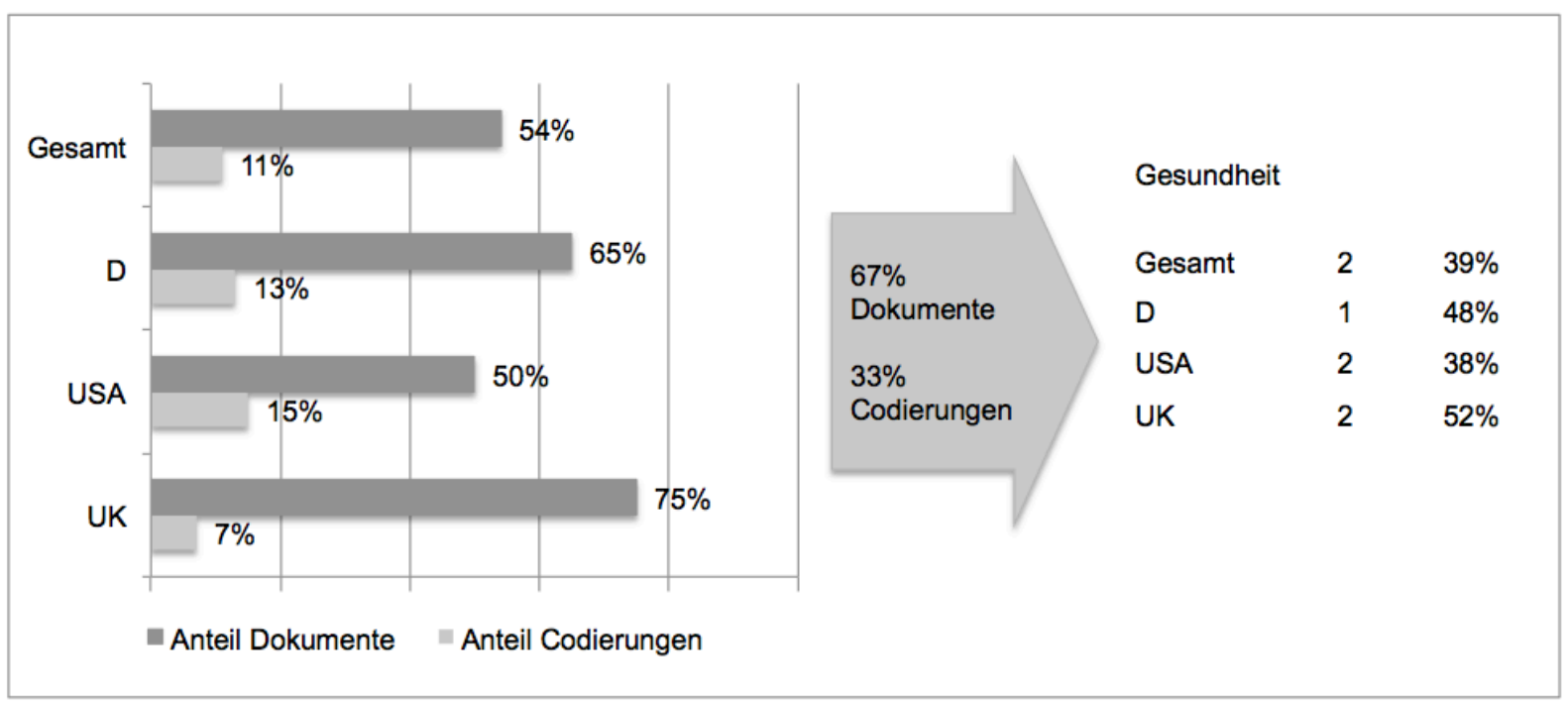

Abbildung 39: Der Wert Gesundheit im Ländervergleich

Abbildung 39 stellt den Wert Gesundheit im Ländervergleich dar. Hierbei wird deutlich, dass Texte aus dem UK einerseits mit einem Anteil Codierungen von 7\% unter den Werten der anderen Länder liegen, aber andererseits mit 75\% den höchsten Anteil Dokumente aufweisen. Insgesamt ergibt sich jedoch kaum ein Unterschied in der Platzierung.

\subsubsection{Zuhause, Eigentum}

Unter den Wert Zuhause, Eigentum fallen zerstörte Häuser, Evakuierung und Sonstiges, was zur temporären oder dauerhaften Nichtnutzbarkeit (oder Einschränkung der Nutzbarkeit) des Zuhauses bzw. des Lebensraumes oder zum Verlust von Eigentum führt. Im Deutschen schließt dies den Begriff von Heimat ein.

Der Wert Zuhause, Eigentum gehört zu den Direct Use Values, ist materiell und ein NichtExistenzialwert.

Die folgenden Beispiele benennen den Verlust des Hauses oder des Eigentums allgemein:

- destroyed houses (Castonguay 2007, 133),

- destroyed villages (Cao 2012, 11),

- destruction of buildings (de Kraker 2013, 12),

- $\quad$ damage to the buildings (Rohr 2003, 93),

- droperty damage (Schott 2002, 119),

- ihr Hab und Gut zurücklassen (Bodner 2010, 87),

- $\quad$ abandoning everything (Bankoff 2007, 42).

Diese Textausschnitte beziehen sich auf weitere Beispiele für den Verlust des Zuhauses, formulieren dies aber eher indirekt:

- für mehrere Monate evakuiert (Bodner 2010, 10), 
- vorübergehend obdachlos (Engels 2009, 13),

- $\quad$ a town ... was relocated (Ross 2014, 87),

- ganze Bevölkerungsgruppen umgesiedelt (Josephson 2010, 3),

- unbewohnbar (Shulmann 2010, 96).

Bei 40\% der Textstellen zum Wert Zuhause, Eigentum erfolgt eine Quantifizierung zumeist anhand der Anzahl der Menschen, die etwas verloren haben oder anhand der Anzahl der beschädigten bzw. zerstörten Häuser (28\% konkrete Angaben, $12 \%$ vage Angaben). Demgegenüber stehen $60 \%$ nicht quantifizierter Fundstellen zum Wert Zuhause, Eigentum.

Abbildung 40 zeigt den Wert Zuhause, Eigentum im Ländervergleich. Wenngleich sich hier keine großen Unterschiede in den Auswertungen nach Anteil Dokumente und Anteil Codierungen zeigen, so werden doch leichte Unterschiede in den Platzierungen insgesamt sichtbar. Während Zuhause, Eigentum in Texten aus Deutschland Rang 2 belegt, erreicht dieser Wert in Texten aus dem UK nur Rang 6. Allerdings weisen beide Platzierungen dabei ähnlich gewichtete Prozentpunkte auf.

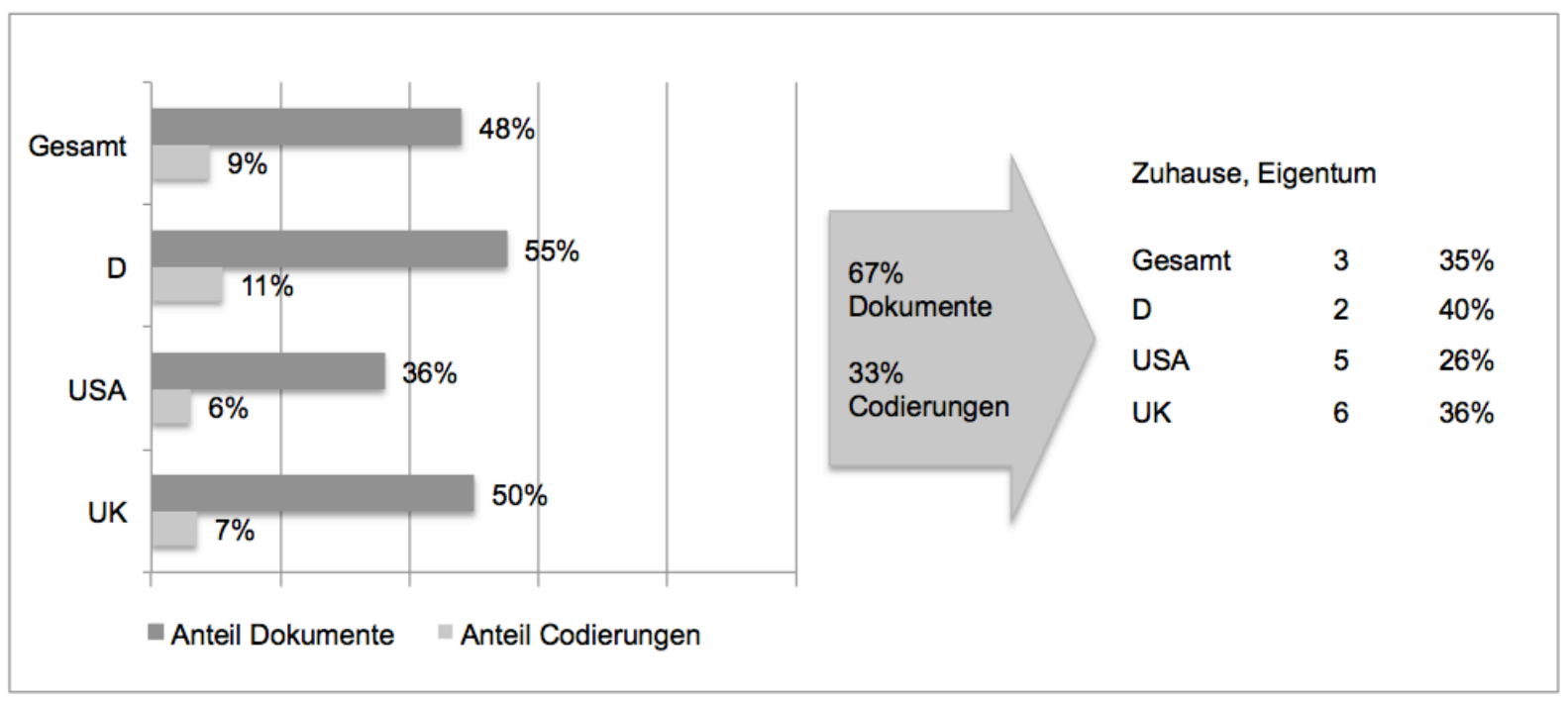

Abbildung 40: Der Wert Zuhause, Eigentum im Ländervergleich

\subsubsection{Boden, Felder}

Der Wert Boden, Felder beschreibt Schäden an Ackerboden wie Erosion, verschlechterte Fruchtbarkeit, Übernutzung, Verwüstung, Wüstenbildung, die zu eingeschränkter Nutzung bis hin zu kompletter Nichtnutzbarkeit führen können. Auch die Minderung oder der Verlust von Arbeitskraft zum Bestellen der Felder (Mensch und Arbeitstiere) sind darin eingeschlossen sowie allgemeine Bodenschädigungen ohne konkreten Bezug zur Nutzung inbegriffen. 
Der Wert Boden, Felder gehört zu den Direct Use Values, ist materiell und ein Existenzialwert (als Unterkategorie von Lebensgrundlage/Nahrung). Die folgenden Textausschnitte beziehen sich auf die negativen Auswirkungen auf landwirtschaftlich genutzte Flächen:

- decreased production capacity of the land (Amate 2013, 116),

- $\quad$ to protect the highly productive agricultural lands (de Kraker 2013, 70),

- the whole area was agriculturally worthless (Mann 1995, 124),

- removes considerable amounts of topsoil (Griggs 2007, 172),

- $\quad$ crop farming was seriously impaired (Brantz 2011, 39),

- $\quad$ wurden ebenso wie große Teile des Ackerlandes ... zerstört (Josephson 2010, 31),

- $\quad$ werden die Felder verwüstet (Bayerl 1989, 252).

Diese Beispiele weisen auf allgemeinere Bodenschädigungen hin:

- $\quad$ land degradation (Beattie 2003, 86),

- $\quad$ hochgradige Bodenschädigungen (Buchholz 2012, 28),

- $\quad$ direct damage to land (Crook 2002, 220),

- Flächen ... verschlammt oder versalzt (Obertreis 2013, 139),,

- Verschwendung von Millionen Hektar Land, die verwüstet und ... kontaminiert wurden (Shulmann 2010, 3),

- desertification (Studnicki 2010, 152).

Darüber hinaus kommen die Stichworte Bodenerosion und Bodenfruchtbarkeit in vielen Texten vor:

- $\quad$ soil erosion (Beattie 2003, 19; Crook 2002, 214; Knopf 2010, 64; Latorre 2001, 14; Mann 1995, 47; Muscolini 2011, 9; Cyffka 2007, 108; Ross 2014, 50; Singh 2000, 380; Slavin 2014, 50; Studnicki 2010, 152),

- $\quad$ reduced or lost fertility (Bankoff 2007, 61; Cao 2012, 91; Mann 1995, 11; Muscolini 2011, 90; Ross 2014, 115; Singh 2000, 311; Slavin 2014, 50; Worster 1988, 22; Völkel 2014, $52)$.

Wenngleich der Wert Boden, Felder einen im wörtlichen Sinne greifbaren Wert darstellt und daher einen hohen Grad an Quantifizierbarkeit vermuten lässt, weisen nur 5\% der Fundstellen zu diesem Wert konkrete quantifizierende Attribute auf und weitere $10 \%$ zeigen zumindest vage quantifizierende Attribute.

Abbildung 41 zeigt den Wert Boden, Felder im Ländervergleich. Auffällig sind hier die Texte aus den USA, die sowohl einen niedrigen Anteil Codierungen (5\%) als auch einen niedrigen Anteil Dokumente (14\%) aufweisen. Daher erreicht dieser Wert bei Texten aus den USA nur Rang 15, bei Texten aus Deutschland und dem UK Platz 3. Insbesondere hinsichtlich der möglichen Langfristigkeit des Schadens („Wenn Boden einmal erodiert ist, dauert es viele 
Tausend Jahre, bis sich an dieser Stelle wieder Boden gebildet hat. “310) verwundert die niedrige Platzierung bei den Texten aus den USA.

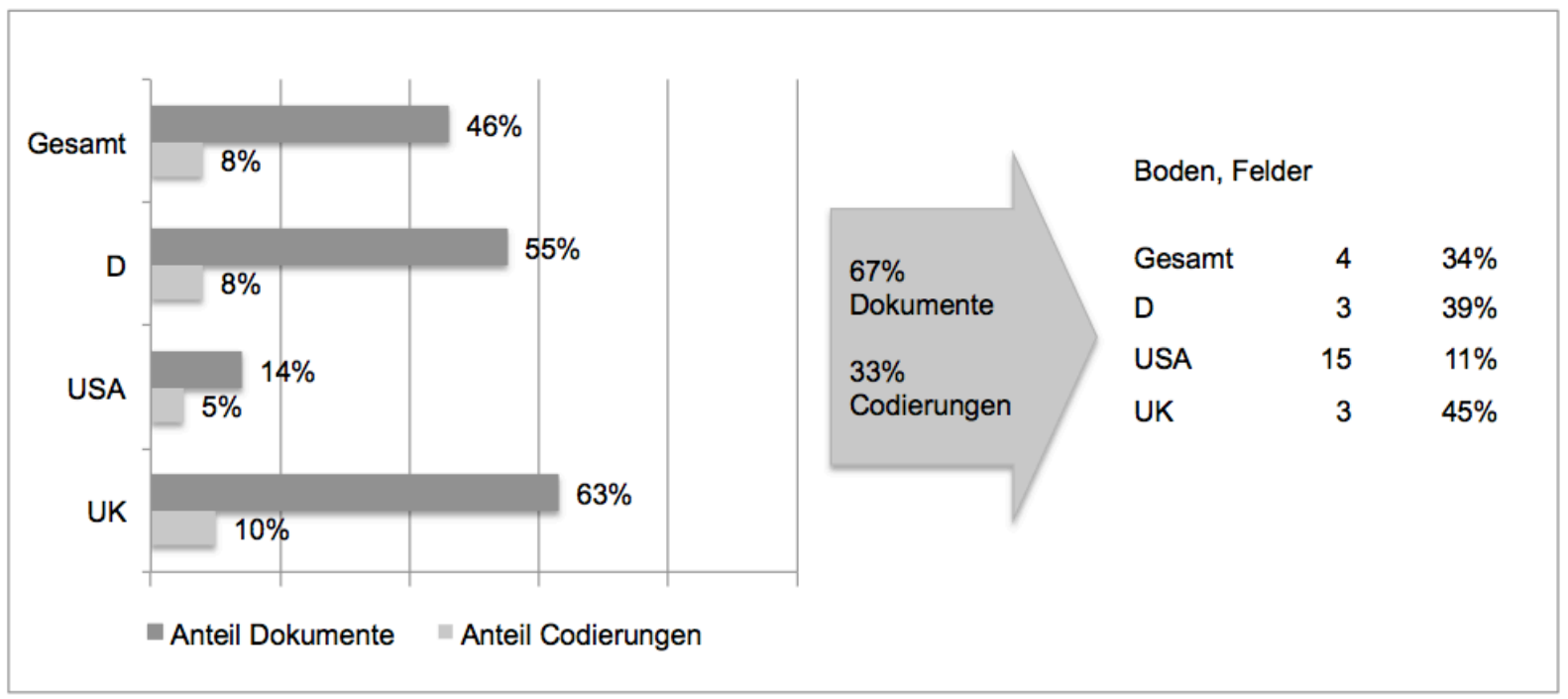

Abbildung 41: Der Wert Boden, Felder im Ländervergleich

\subsubsection{Leben}

Textstellen, die menschliche Todesopfer beschreiben, werden unter den Wert Leben subsumiert. Hier werden Fundstellen eingeordnet, in denen explizit vom Tod berichtet wird; potenziell tödliche Krankheiten hingegen werden dem Wert Gesundheit zugeordnet.

Der Wert Leben gehört zu den Direct Use Values, ist weder materiell noch immateriell und ein Existenzialwert. Die folgenden Textausschnitte zeigen Beispiele für den allgemeinen Hinweis auf menschliche Todesopfer:

- $\quad$ killed people (Cao 2012, 11),

- massive in terms of casualties (de Kraker 2013, 410),

- increased the heat related deaths (Mann 1995, 120),

- $\quad$ suffered relatively few casualties (Smith 2012, 252),

- massenhaftem Hungertod (Bowlus 1988, 74),

- $\quad$ sehr geringe Lebenserwartung (Bayerl 1989, 114),

- $\quad$ Kindersterblichkeit (Josephson 2010, 81; Obertreis 2013, 25).

In diesen Beispielen zeigt sich der Wert Leben über die Nennung der Opferzahlen:

- $\quad$ Nennung der Anzahl der Toten (Aguirre 2012, 36; Bankoff 2007, 43; Bruno 2013, 9; Cao 2012, 20; Carey 2007, 30; Castonguay 2007, 117; Engels 2009, 10; Huff 2010, 38; Kraft

\footnotetext{
${ }^{310}$ Verena Winiwarter/Hans-Rudolf Bork, Umweltgeschichte: ein Plädoyer für Rücksicht und Weitsicht: [Vortrag im Großen Sendesaal des ORF-RadioKulturhauses am 20. März 2014], Wien, 2014, S. 58.
} 
2013, 26; Schenk 2010, 41; Muscolini 2011, 135; Rohr 2003, 201; Schott 2002, 90; Sheail 2013, 45; Worster 1988, 10; Bowlus 1988, 7).

Der Wert Leben wird bei 52\% aller Fundstellen quantifiziert und ist damit der mit Abstand am häufigsten konkretisierte Wert (Zuhause, Eigentum folgt mit 28\%). Zudem zeigen weitere 14\% der Textstellen zumindest vage Quantifizierungen wie z.B. „massive death toll“ oder „tens of thousands“.

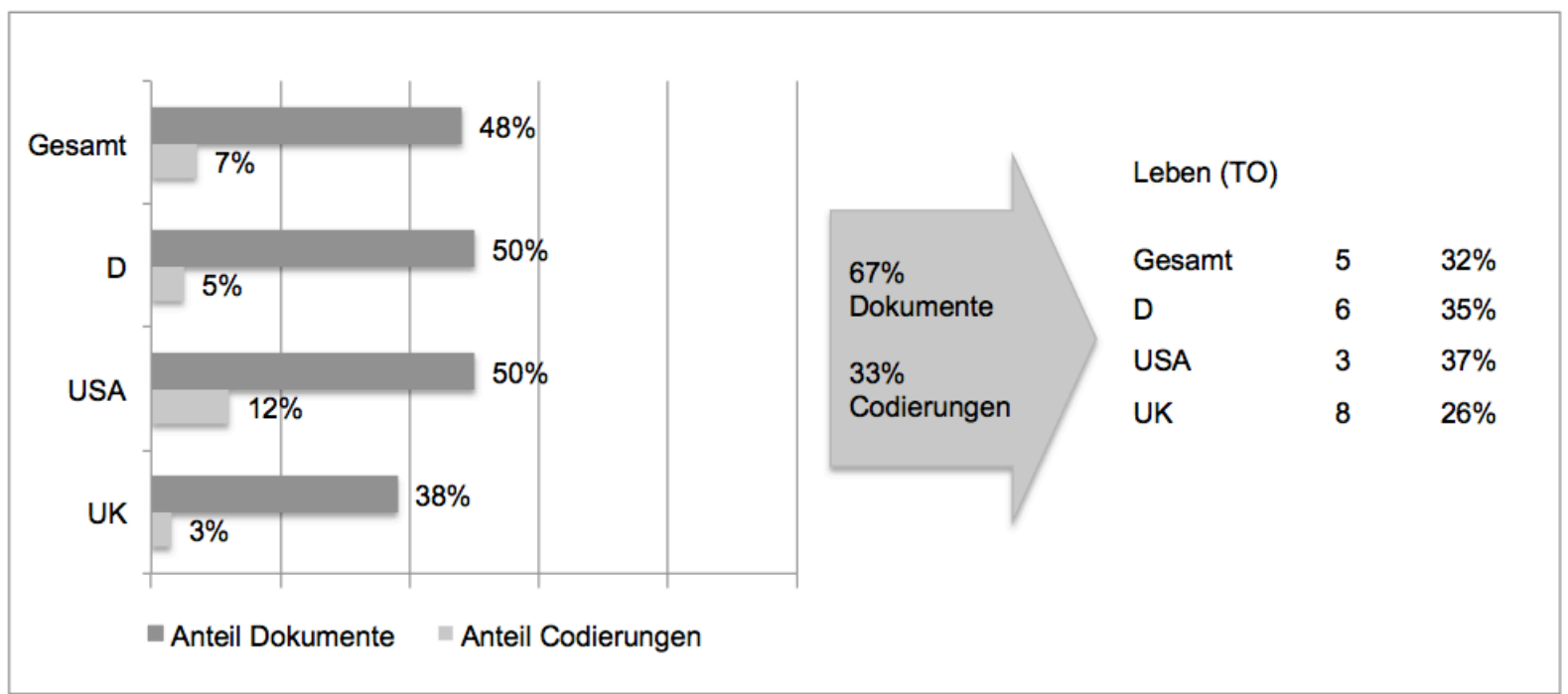

Abbildung 42: Der Wert Leben im Ländervergleich

Abbildung 42 stellt den Wert Leben im Ländervergleich dar. Hier gibt es eine Abweichung zwischen den Texten aus den USA und denen aus dem UK: Während in Texten aus den USA der Wert Leben mit einem Anteil Codierungen von 12\% auf Rang 3 landet (wie auch nach Anteil Dokumente sowie insgesamt), weisen Texte aus dem UK nur einen Anteil Codierungen von 3\% auf, wodurch der Wert Leben nur Rang 8 erreicht (wie auch nach Anteil Dokumente sowie insgesamt). Insgesamt gibt es zwar kleine Unterschiede in der Platzierung, die gewichteten Prozentpunkte liegen jedoch in ähnlichen Bereichen.

\subsubsection{Nahrungsmittel}

Der Wert Nahrungsmittel beschreibt die eingeschränkte oder nicht vorhandene Verfügbarkeit und Qualität von Nahrungsmitteln. Eingeschränkte Verfügbarkeit zeigt sich auch durch Preissteigerungen. Nahrungsmittel meint Nahrung für den Menschen. Wird im Text hingegen über eine Hungersnot, also die Folge von Nahrungsmangel berichtet, wird der Sachverhalt bei Gesundheit/Hunger eingeordnet. Werden im Text die gesundheitlichen Folgen des Hungers behandelt, wird die Fundstelle dem Wert Gesundheit zugeordnet. 
Der Wert Nahrungsmittel gehört zu den Direct Use Values, ist materiell und ein Existenzialwert. Diese Textauszüge beziehen sich auf die allgemein eingeschränkte Verfügbarkeit von Nahrungsmitteln:

- $\quad$ Tiere ausgerottet, von denen sehr viele den Menschen als Speise dienen (Bayerl 1989, 252),

- food shortages (Brantz 2011, 117),

- Versorgungsproblemen (Knopf 2010, 39),

- $\quad$ not even have maize to eat (Perri 2009, 93),

- food sources (Slavin 2014, 67),

- a catastrophic dearth of food (Slavin 2014, 36),

- $\quad$ remaining food stocks (Smith 2012, 402).

Die folgenden Beispiele weisen auf bestimmte qualitative und quantitative Einschränkungen in der Ernährung hin:

- $\quad$ wurde ... von den Anwohnern durch die Milch der Kühe aufgenommen (Shulmann 2010, $61)$,

- $\quad$ die für ihre Ernährung wichtigen Fischbestände (Josephson 2010, 67),

- $\quad$ sources of protein (Bankoff 2007, 17),

- $\quad$ animal protein (Studnicki 2010, 115).

Diese Textbeispiele beziehen sich indirekt auf eine eingeschränkte Verfügbarkeit oder Qualität von Nahrungsmitteln:

- $\quad$ Fischerei-Industrie am Aralsee ging zugrunde (Obertreis 2013, 19),

- $\quad$ tailings damaged shad fisheries (Ross 2014, 87),

- $\quad$ in der Nahrungskette anzureichern (Scheringer 2012, 64),

- $\quad$ nicht mehr für die Rentierzucht geeignet (Josephson 2010, 59),

- $\quad$ loss of livestock (de Kraker 2013, 12),

- Nahrungskette des Menschen (Rohr 2007, 4),

- $\quad$ stiegen ... die Preise für Getreide und Brot (Huff 2010, 140),

- $\quad$ contaminated milk (Buhs 2002, 75).

Der Wert Nahrungsmittel weist nur bei $8 \%$ der Fundstellen zu diesem Wert konkrete quantifizierende Attribute auf und bei weiteren $5 \%$ vage quantifizierende Attribute.

Abbildung 43 zeigt den Wert Nahrungsmittel im Ländervergleich. Sichtbar wird hier ein großer Unterschied zwischen Texten aus den USA und Texten aus dem UK. Während die Codierungen ähnliche Anteile aufweisen, liegen die Werte bei Anteil Dokumente weit auseinander. Insgesamt erreicht der Wert Nahrungsmittel daher bei Texten aus den USA nur 
Platz 12 (mit nur 15 Prozentpunkten), während der Wert bei Texte aus dem UK Rang 4 erreicht (mit 43 Prozentpunkten).

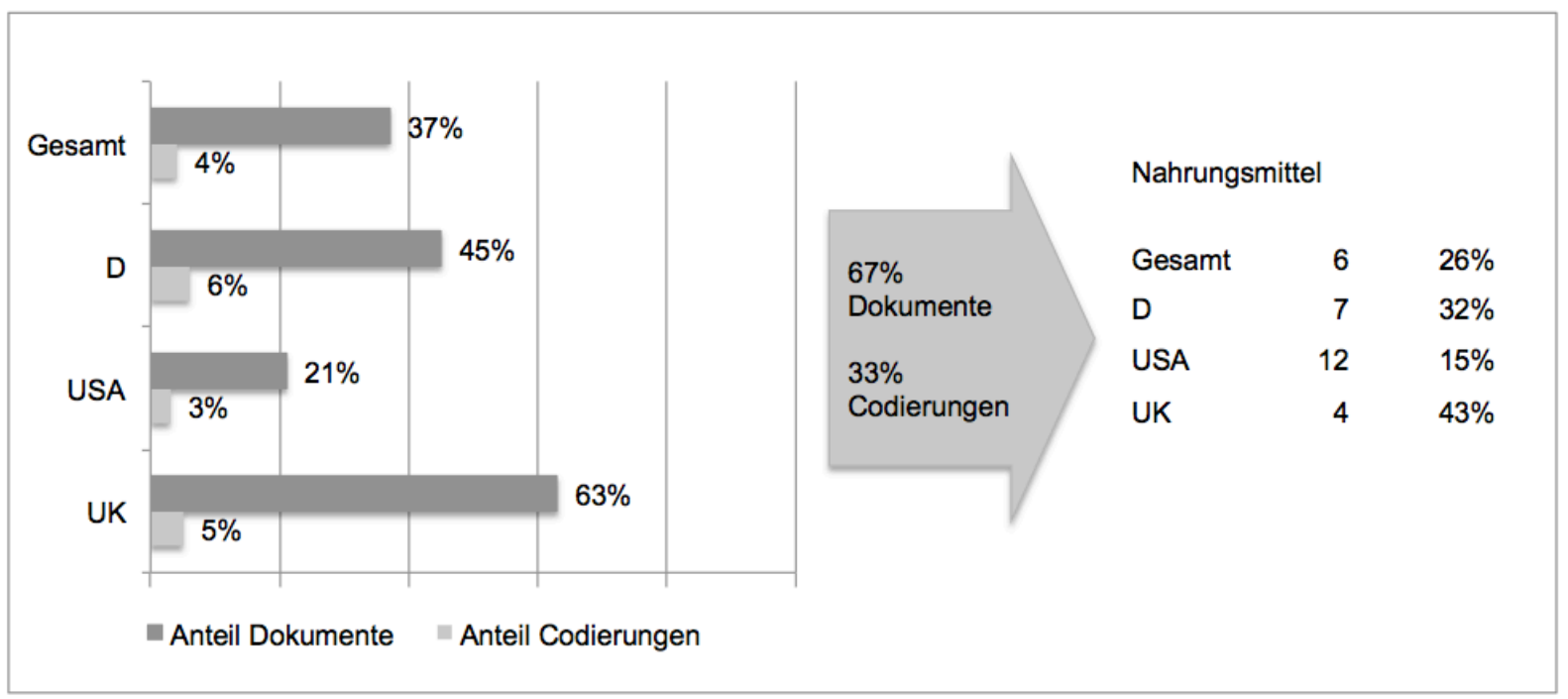

Abbildung 43: Der Wert Nahrungsmittel im Ländervergleich

\subsubsection{Einkommensquelle}

Der Wert Einkommensquelle bezeichnet die Verringerung oder den Verlust von Naturerzeugnissen, die für einzelne Produzenten oder Individuen eine Einkommensquelle darstellen. Dies schließt auch eingeschränkte oder nicht vorhandene Möglichkeiten der Produktion, der Ernte oder des Verkaufs ein. Sind viele Produzenten (Industrien) oder ganze Regionen betroffen, wird der Sachverhalt bei dem Wert Ökonomie eingeordnet.

Der Wert Nahrungsmittel gehört zu den Direct Use Values, ist materiell und ein Existenzialwert. Die folgenden Beispiele weisen auf Arbeitslosigkeit oder die Gefährdung der Existenzgrundlage (des Einkommens) hin:

- $\quad$ were without work (Castonguay 2007, 94),

- $\quad$ left them unemployed (Pritchard 2012, 70),

- $\quad$ threatening the livelihoods (Carey 2007, 118),

- $\quad$ who depended upon forestry for their livelihoods (Mathews 2003, 184),

- $\quad$ while they waited for the oyster beds to recover (Perri 2009, 93),

- $\quad$ so daß die Mühle zum Stillstand kam (Bayerl 1989, 477),

- $\quad$ Farmer lebten von der Fürsorge (Worster 1988, 11).

Diese Textstellen beziehen sich speziell auf Einkommensverluste im Agrarsektor:

- destroyed the harvests (Bankoff 2007, 42),

- difficulties facing many farmers (Beattie 2003, 80),

- $\quad$ many farmers ... were ruined (Brantz 2011, 39), 
- $\quad$ one farm lost fifteen cows and calves (Buhs 2002, 7).

Diese Ausschnitte sind weitere Beispiele für Einschränkungen bei der Einkommensquelle:

- Kompromiss zwischen Verhütung langfristiger Bodenschädigung und Maximierung des kurzfristigen Einkommens (Knopf 2010, 72),

- $\quad$ betrachteten die Natur ... als wirtschaftliches Potenzial (Josephson 2010, 94),

- $\quad$ impinging on ... tourism (Carey 2007, 109),

- a collapse in olive oil prices (Amate 2013, 137),

- $\quad$ profits from the sale of wood (Crook 2002, 126).

80\% der Fundstellen zum Wert Einkommensquelle enthalten keine quantifizierenden Attribute. Dagegen weisen nur $14 \%$ der Textstellen konkrete und weitere $6 \%$ vage Beschreibungen auf.

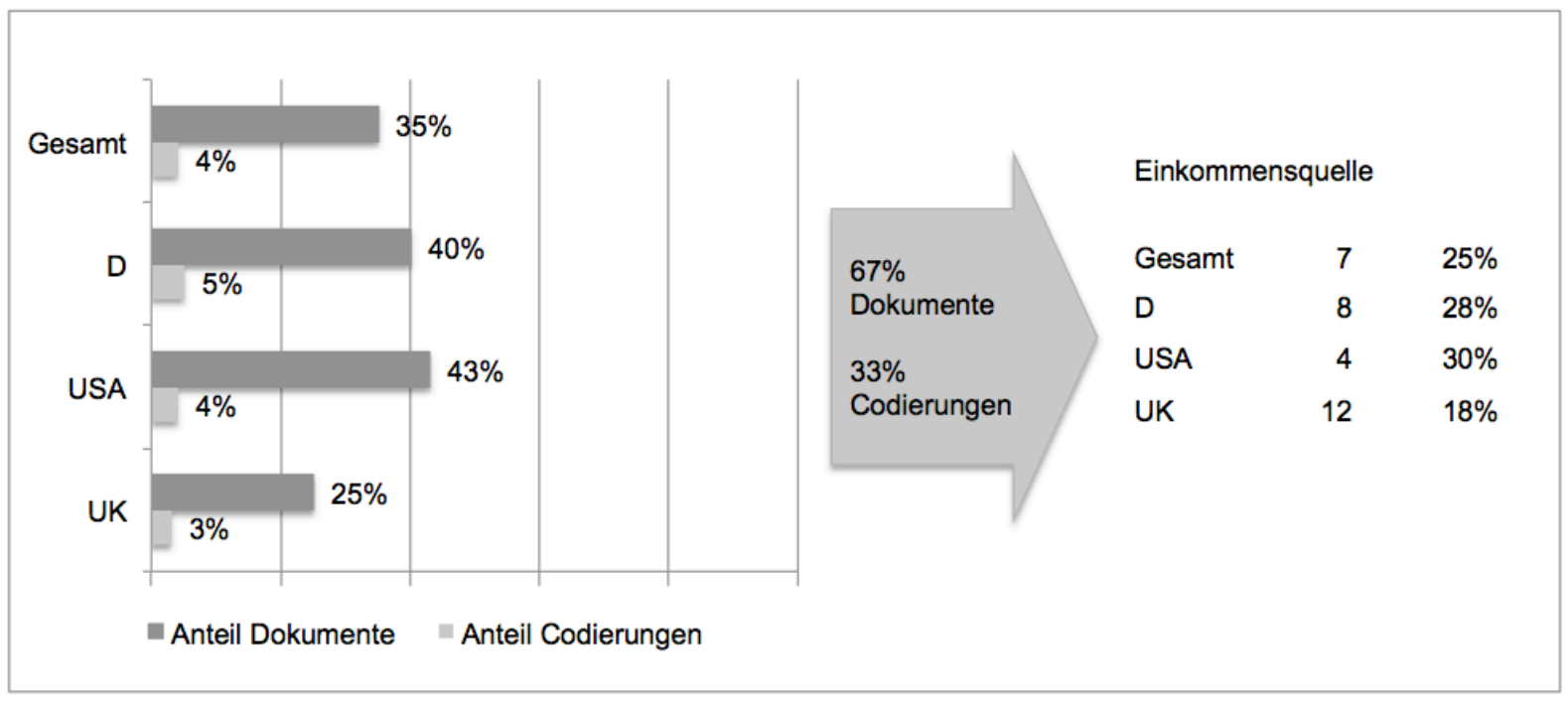

Abbildung 44: Der Wert Einkommensquelle im Ländervergleich

Abbildung 44 zeigt den Wert Einkommensquelle im Ländervergleich. Hier gibt es einen größeren Unterschied zwischen den Texten aus den USA und Texten aus dem UK. Bei Letzteren kommt der Wert Nahrungsmittel nur in 25\% der Dokumente vor und erreicht damit insgesamt nur Platz 12 während er bei Texten aus den USA Platz 4 belegt.

\subsubsection{Wasser}

Der Wert Wasser beschreibt die Beeinträchtigung von Wasserqualität bzw. -verfügbarkeit. Hinsichtlich der Art des Wassers (Trinkwasser, Meerwasser, Grundwasser, etc.) gibt es keine Einschränkungen.

Der Wert Wasser gehört zu den Direct Use Values, ist materiell und ein Existenzialwert. Die nachfolgenden Textausschnitte beziehen sich auf eine eingeschränkte Wasserqualität: 
- Wasserqualität (Josephson 2010, 24),

- Verschmutzung der Gewässer (Völkel 2014, 52),

- $\quad$ water quality (Mc Farlane 2012, 14),

- zur Trinkwassergewinnung ungeeignet (Cyffka 2007, 74),

- Grundwasser ... kontaminiert (Shulmann 2010, 3),

- Defizite der ... Gewässerreinhaltung (Bayerl 2009, 94),

- Wasservergiftung (Bayerl 1989, 110),

- exploitation of groundwater (Latorre 2001, 154),

- $\quad$ harming the water supply (Mann 1995, 61),

- damage the springs (Mathews 2003, 151).

Diese Textbeispiele bringen eine eingeschränkte Wasserverfügbarkeit zum Ausdruck:

- dry rivers (Singh 2000, 313),

- Trockenfallen von Brunnen (Buchholz 2012, 42),

- $\quad$ impinging on drinking water supplies (Carey 2007, 109),

- Wassermangel (Obertreis 2013, 156).

Für den Wert Wasser wurden nur bei 13\% der Fundstellen zu diesem Wert konkrete quantifizierende Attribute gefunden und bei weiteren $8 \%$ vage quantifizierende Attribute.

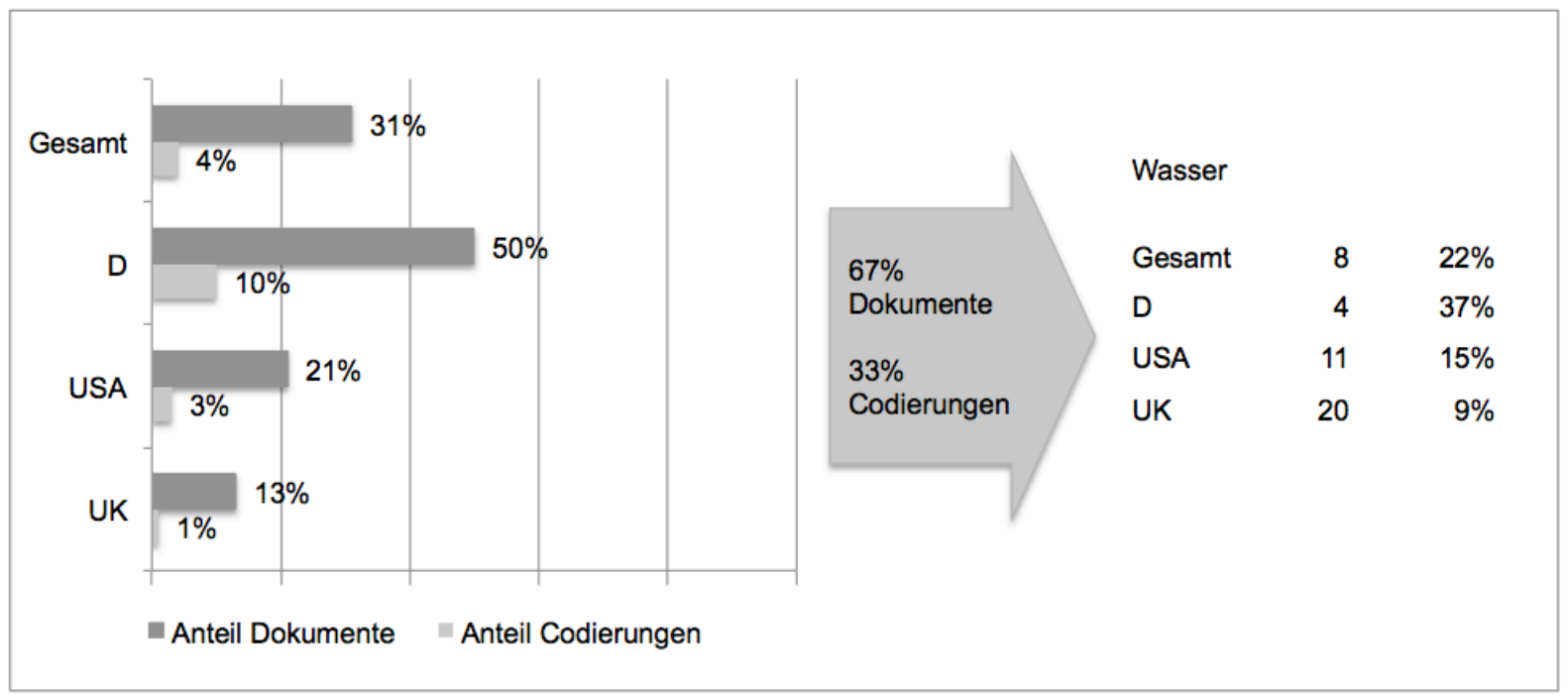

Abbildung 45: Der Wert Wasser im Ländervergleich

Abbildung 45 stellt den Wert Wasser im Ländervergleich dar. Hier zeigen sich sehr große Unterschiede zwischen den drei dargestellten Ländern. Die niedrige Platzierung in Texten aus den USA und dem UK ist dabei jedoch nicht in den behandelten Themen begründet, im Gegenteil: nur ein Text aus dem UK bietet sich nicht direkt für den Wert Wasser an (Thema: Korallen sammeln). Bei den Texten aus den USA würde es sich bei mindestens weiteren $43 \%$ anbieten den Wert Wasser zu erwähnen. Andererseits sind die Themen der Texte aus 
Deutschland weder mehr noch weniger geeignet den Wert Wasser zu integrieren. Trotzdem erreicht der Wert bei Texten aus Deutschland insgesamt Rang 4, während er bei Texten aus dem UK nur Rang 20 (von insgesamt 28) belegt. 


\section{Zusammenfassung und Diskussion}

\subsection{Zusammenfassung}

Die vorliegende Arbeit leistet einen klärenden Beitrag zu der Diskussion, welche Wertvorstellungen in umwelthistorischen Texte dominieren. Dafür wird umwelthistorisches Textmaterial hinsichtlich des Verständnisses von Umweltschäden untersucht. Das Textmaterial reicht von regionalen über universale Kontexte und von ephemeren Ereignissen bis hin zu langfristigen Abläufen. Das Ziel der Untersuchung ist eine deskriptive Aussage zu diesen Werten, wobei die Analyse der Texte der entdeckenden Forschungslogik folgt. Auf die einem Text zugrundeliegenden impliziten Werte kann nur indirekt geschlossen werden. Dies erfolgt mithilfe eines Indikators, der auf bestimmte Werthaltungen hinweisen kann. Der Begriff „Umweltschaden“ ist hierfür besonders gut geeignet, da ein Schaden unerwünschte Folgen eines Ereignisses bezeichnet und die Gründe für diese Unerwünschtheit in den zugrundeliegenden Werten zu finden sind. Auf die übliche Unterscheidung von Wert im ökonomischen Sinn und Werten im ideellen Sinn wird bei der Textanalyse bewusst verzichtet, bzw. es wird keine Kategorie ausgeschlossen, um ein möglichst vollständiges Bild zu erhalten. Als Methode für die Untersuchung wurde die Qualitative Inhaltsanalyse ausgewählt, da sie annimmt, dass Menschen ihre Ansichten und Einstellungen, d. h. ihre Werthaltungen, in dem zum Ausdruck bringen was sie sagen oder schreiben. Zunächst wird in den zu analysierenden Texten nach Indikatoren gesucht. Anschließend werden durch Interpretation der Texte zugrunde liegende Werte identifiziert und in ein Kategoriensystem eingeordnet, das induktiv erarbeitet wird.

Vor der Textanalyse werden zunächst die theoretischen Hintergründe dargestellt. Umweltgeschichte bildete sich in den 1970er-Jahren heraus und untersucht die Wechselwirkungen zwischen Gesellschaft und Natur. Daraus ergibt sich ein interdisziplinärer Arbeitsansatz. Die Positionierung an der Schnittstelle zwischen Natur- und Geisteswissenschaften ist Chance und Schwierigkeit zugleich. Erste Forschungsarbeiten wurden in den USA veröffentlicht, es folgte 1977 die Gründung des ASEH, 1999 entstand das europäische Pendant ESEH. Umweltgeschichte bietet eine sehr große Auswahl möglicher Themen, gleichzeitig fehlt noch der größere Kontext, der die verschiedenen Themen zusammenfasst. Diese Tatsache scheint auch die Einordnung der Umweltgeschichte in die bestehende Wissenschaftslandschaft zu erschweren. Somit ist die Institutionalisierung der Umweltgeschichte noch nicht abgeschlossen. Themen der umweltgeschichtlichen Forschung wie z. B. die Rekonstruktion von Naturwahrnehmung oder die Analyse interaktiver Prozesse zwischen Mensch und Natur erfordern eine Kombination von natur- und geisteswissenschaftlichen Methoden. Das heißt, die Kenntnis und die Anwendung verschiedenster 
Methoden aus unterschiedlichsten Disziplinen stellen hohe Anforderungen an die in diesem Bereich Forschenden.

Geschichtswissenschaft scheint nach wie vor nach möglichst viel Objektivität zu streben, Wahrnehmung und Interpretation sind jedoch sehr individuelle Vorgänge. So wundert es nicht, dass das Thema Objektivität der Geschichtswissenschaft einige Schwierigkeiten bereitet und dass sich die Wertefrage durch die gesamte (westliche) Geschichtsschreibung zieht. Diese betrifft aber nicht nur die Geschichtswissenschaften, sondern auch andere Disziplinen; hauptsächlich die der Geisteswissenschaften. Es gibt bis heute Diskussionen, ob Objektivität in der Geschichtswissenschaft möglich (und auch erstrebenswert) ist. Werte beeinflussen den gesamten Forschungsprozess, Forscher sollten sich daher ihrer eigenen Werte bewusst sein, um deren Einfluss auf ihre Forschungsarbeit besser einschätzen (und begrenzen) zu können. Zugunsten der intersubjektiven Nachvollziehbarkeit sollten diese Werte bzw. der Prozess der Bewertung zudem anderen Forschern zugänglich gemacht werden. Die Interpretation von Quellen unter Einbezug von Werten ist eine schwierige und komplexe Aufgabe. Zudem können umweltgeschichtliche Themen auch Analysen über längere Zeiträume erfordern, innerhalb derer sich die Werte der Akteure ändern können. Auch das Ergebnis der Forschungsarbeit, bzw. die Schlussfolgerung daraus wird von Werten beeinflusst. Häufig steht umweltgeschichtliche Forschung im Fokus der Öffentlichkeit, was zu Bewertungen bzw. einer öffentlichkeitswirksamen Formulierung der Schlussfolgerungen führen kann. Die Praxisrelevanz ergibt sich aus den daraus ggfs. abgeleiteten Handlungsempfehlungen. Typische Fehlschlüsse ${ }^{311}$ sowie die Vermischung von Politik und Wissenschaft sind hier zu vermeiden.

Zusammenfassend lässt sich feststellen, dass die Problematik der Wertfreiheit sehr intensiv und schon über einen langen Zeitraum diskutiert wird. Es scheint weitgehend Einigkeit darüber zu herrschen, dass Objektivität in der Geschichtswissenschaft weder möglich noch zielführend ist. Trotzdem dürfte es hilfreich sein, wenn sich die Forscher der Werteproblematik bewusst sind.

Im textanalytischen Teil der vorliegenden Arbeit wurden 54 Texte analysiert, von denen 20 in Deutsch und 34 in Englisch verfasst sind. Sämtliche Indikator- und Wertefundstellen wurden in den Dokumenten markiert und in Kategorien organisiert. Es gibt 5 Indikatoren ${ }^{312}$ und insgesamt 28 Werte. An die Definition und ggfs. Abgrenzung der Werte und Wertekategorien schließt sich die Auswertung der erhobenen Metadaten der Texte an. Veröffentlicht wurden die Aufsätze im Zeitraum von 1988 bis 2014, wobei drei Viertel der Texte 2007 oder später publiziert wurden. Die drei am häufigsten vertretenen Länder sind Deutschland (20 Texte),

\footnotetext{
${ }^{311}$ Vgl. Fußnote 160, S. 36.

${ }^{312}$ Alle Indikatorwörter müssen im Sinne von Umweltschaden verwendet werden.
} 
USA (14) und UK (8). 70\% der Dokumente wurden Zeitschriften entnommen, 30\% stammen aus Sammelbänden.

Es folgen einige Auswertungen zu den Indikatorcodierungen. Die meisten Fundstellen (147) hat das Wort "Katastrophe“, während das Wort „Umweltzerstörung“ in den meisten Dokumenten (37) vorkam. Der Begriff „Umweltschaden“ erzielte 35 Treffer in 16 Dokumenten. Beim dann folgenden Vergleich der Anzahl Indikatorcodierungen mit der Anzahl Wertecodierungen zeigt sich insgesamt nur ein schwacher Zusammenhang. Tendenziell gibt es umso mehr Wertecodierungen, je mehr Indikatorcodierungen es gibt. Eine Verbindung zum Jahr der Veröffentlichung lässt sich nicht herstellen. Durchschnittlich enthält jeder Text 16 Wertecodierungen und neun Indikatorcodierungen. Englische Texte enthalten mehr Wertecodierungen (17) als Deutsche Texte (12). Den höchsten Durchschnittswert erzielen Texte aus dem UK mit 24 Fundstellen pro Text. Während Beiträge aus Sammelbänden nur durchschnittlich 10 Wertecodierungen aufweisen, enthalten Texte aus Zeitschriften im Schnitt 15 Wertecodierungen. Beiträge aus den beiden Zeitschriften „Environment and History“ sowie „Environmental History“ kommen auf durchschnittlich 19 Wertecodierungen. Ein weiterer Vergleich zeigt die Anzahl der Wertecodierungen einzelner Werte in Abhängigkeit von der Nennung des Indikators „Umweltschaden“. Insgesamt ist kein direkter Zusammenhang erkennbar, vielmehr wird deutlich, dass die explizite Benennung eines Phänomens als „Umweltschaden“ weder einen Rückschluss auf die Anzahl der Wertefundstellen noch auf die vorgefundenen Werte zulässt. Wird die Bezeichnung „Umweltschaden“ allerdings nicht im Text verwendet, weisen bestimmte Werte eine deutlich höhere Anzahl an Wertecodierungen auf („Ökonomie“, „Zuhause, Eigentum“, „Tod“, „Schönheit der Natur“, „Tiere“, „Unberührte Natur“, „Infrastruktur“, „Erholung, Freizeit, Komfort“). Dagegen weisen manche Werte mehr Wertecodierungen auf, wenn der Indikator „Umweltschaden“ genannt wird (u. a. „Wasser“, „Nachhaltigkeit“, „Imageschaden“, „Hunger“, „Luft“). Schließlich wird in diesem Abschnitt untersucht, wie viele Wertecodierungen quantifizierende Attribute aufweisen und zu welchen Werten sie gehören. Leben, Ökonomie und Zuhause, Eigentum sind die drei Werte mit den meisten Quantifizierungen. Insgesamt enthalten nur $23 \%$ der Wertecodierungen quantifizierende Attribute. Diese verteilen sich jedoch über $76 \%$ der untersuchten Dokumente, so dass 24\% der Dokumente keine Quantifizierungen aufweisen.

Etwa ein Viertel der Texte beschreibt Ereignisse mit einer Dauer von weniger als einem Jahr, ungefähr die Hälfte hat eine Dauer von 1 bis 100 Jahren und ein weiteres Viertel eine Dauer von mehr als 100 Jahren. Bezogen auf die Schadensdauer zeigen ca. 30\% der Texte eine Dauer von weniger als 10 Jahren, weitere $40 \%$ eine Dauer von 10 bis 100 Jahren und die restlichen $30 \%$ eine Schadensdauer von mehr als 100 Jahren. Ereignis- und Schadensfläche sind in knapp $80 \%$ der Texte identisch, bei $20 \%$ ist die Schadensfläche größer. Beim Vergleich von Ereignisdauer und -ausdehnung zeigt sich, dass $44 \%$ aller Dokumente 
Phänomene untersuchen, die eine Ereignisausdehnung zwischen 1.000 und $10.000 .000 \mathrm{~km}^{2}$ sowie eine Ereignisdauer unter 150 Jahren haben. 61\% aller Texte behandeln vom Menschen verursachte Schäden.

Des Weiteren wurde die Verteilung der Codierungen in den einzelnen Dokumenten betrachtet. Bei der Mehrheit der Dokumente (69\%) sind die Codierungen über das gesamte Dokument verteilt. Bei weiteren $26 \%$ ist bei der Verteilung ein Schwerpunkt zu erkennen, z. B. Codierungen nur in der ersten Hälfte des Dokuments, aber die Codierungen zeigen noch eine flächige Verteilung. Wenn die Codierungen über das Dokument verteilt sind, spricht das meines Erachtens dafür, dass die markierten Werte eher unbewusst in den Text eingeflossen sind. Abschließend werden Anmerkungen zu einzelnen Texten unter verschiedenen Stichworten zusammengefasst, wobei u. a. einige Auffälligkeiten zum anthropozentrischen Ansatz aufgezeigt werden und an verschiedenen Beispielen belegt wird, dass der jeweils behandelte Umweltschaden nicht eindeutig oder zu gering beschrieben wird.

Anschließend werden die einzelnen Werte zu folgenden Wertearten zusammengefasst und es wird eine Auswertung nach Anzahl Codierungen sowie nach Anzahl der Dokumente sowohl insgesamt als auch nach Ländern (Deutschland, USA, UK) vorgenommen: anthropozentrisch/nicht-anthropozentrisch, materiell/immateriell, Existenzialwerte/Nicht-Existenzialwerte sowie Use Values/Non-Use Values. Die Ergebnisse dieser Auswertungen werden in Abschnitt 6.2 zusammengefasst.

Das dann folgende fünfte Kapitel beinhaltet schließlich die Auswertungen, die die Eingangsfrage welche Werte in umweltgeschichtlichen Texten vorherrschen, beantworten. Dafür wird zunächst die Auswertungsmethode erläutert, dann eine länderspezifische Betrachtung für die meistvertretenen Länder Deutschland, USA und UK durchgeführt um schließlich die insgesamt bestimmenden Werte zu ermitteln. Das Ergebnis wird ebenfalls im folgenden Abschnitt zusammengefasst. Schließlich werden die als dominierend identifizierten Werte im Einzelnen diskutiert. Nach der Definition und Einordnung des Wertes bzgl. der Werteart und kategorie wird das Wertverständnis anhand von Textausschnitten vertieft und werden ggfs. Besonderheiten aufgezeigt. 


\subsection{Ergebnis}

Welche Werte dominieren in umweltgeschichtlichen Texten? Diese Frage wurde anhand der Verwendung des Begriffs Umweltschaden bearbeitet. Basierend auf der durchgeführten Untersuchung wurden die folgenden Werte als dominierend ermittelt:

1. Ökonomie

2. Gesundheit

3. Zuhause, Eigentum

4. Boden, Felder

5. Leben

6. Nahrungsmittel

7. Wasser

8. Einkommensquelle

Alle acht Werte sind anthropozentrisch geprägt und nutzungsabhängige Werte (Direct Use Values). Bis auf Gesundheit und Leben, die weder als materiell noch als immateriell eingestuft werden, gehören alle Werte zu den materiellen Werten. Fünf der Werte gehören zu den Existenzialwerten: Gesundheit; Boden, Felder; Leben; Nahrungsmittel und Wasser.

Insgesamt beziehen sich $82 \%$ aller Wertecodierungen auf anthropozentrische Werte und 98\% der untersuchten Dokumente enthalten anthropozentrische Werte (nicht-anthropozentrische Werte sind in 59\% der Dokumente zu finden). Es sind also anthropozentrische Werte, die vorherrschen. Des Weiteren sind die dominierenden Werte nutzungsabhängig. Insgesamt betreffen $74 \%$ aller Wertecodierungen Direct Use Values, zusammen mit den Indirect Use Values kommen diese Werte sogar auf einen Anteil von 91\%. Direct Use Values sind in $98 \%$ aller untersuchten Dokumente zu finden (Non-Use Values sind in $39 \%$ der Dokumente vertreten). Wenngleich die Gruppe der materiellen Werte die Mehrheit der bestimmenden Werte stellt, erzielen diese insgesamt nur einen Anteil von $51 \%$ an der Summe aller Wertecodierungen. Allerdings sind materielle Werte in $94 \%$ aller betrachteten Dokumente enthalten. Werte der Kategorie Gesundheit sind in 67\% der Dokumente vertreten. Auch die Gruppe der Existenzialwerte erreicht einen hohen Verbreitungsgrad: diese Werte kommen in $89 \%$ aller untersuchten Dokumente vor.

Bezüglich der drei am häufigsten vertretenen Länder Deutschland, USA und UK gibt es einige länderspezifische Besonderheiten. Betrachtet man nur Texte aus Deutschland, sind es dieselben acht Werte, die insgesamt als dominierend angesehen werden, allerdings in veränderter Platzierung, wobei der Wert Ökonomie auf Platz 5 am stärksten abweicht. Bei Texten aus den USA sind nur fünf dominierende Werte identifiziert worden, die alle in den o. g. insgesamt führenden Werten enthalten sind. Die beiden größten Abweichungen gibt es bei den Werten Boden, Felder und Nahrungsmittel, die nur die Plätze 15 und 12 erreichen. 
Die Anzahl der bestimmenden Werte bei Texten aus dem UK liegt mit zehn relativ hoch, wobei mit dem Wert Tiere auf Rang 5 ein erster nicht-anthropozentrischer Wert auftaucht. Die andere große Abweichung liegt in der Platzierung des Wertes Wasser, der nur Platz 20 erreicht.

\subsection{Diskussion}

Die Textanalyse anhand des Begriffs Umweltschaden hat sich als sinnvolles Verfahren erwiesen; auch implizite Werte konnten mit dieser Methode herausgefiltert werden. Ebenfalls bewährt hat sich die induktive Kategorienbildung, bedingte sie doch eine offene Herangehensweise. Zusammen mit der Anwendung des Begriffs "Wert" sowohl im moralischen als auch im ökonomischen Sinn konnte so ein möglichst vollständiges Bild der in den Texten enthaltenen Werte ermittelt werden. Zum Begriff Umweltschaden ist anzumerken, dass er dem Bereich „umwelthygienischer Ansatz“ zuzurechnen ist. Dieser besagt, dass eine intakte Natur durch den Menschen gestört bzw. geschädigt wird. ${ }^{313}$ Darüber hinaus befasst sich Umweltgeschichte allerdings auch mit weiteren Fragestellungen und so stellt auch Radkau fest, dass Umweltgeschichte nicht nur aus Katastrophen besteht. ${ }^{314}$ Daher könnte nun ein nächster Schritt darin bestehen, zu überprüfen, ob die hier verwendete Methode auch auf Texte ohne den Begriff Umweltschaden anwendbar ist.

Bestätigt hat sich die Aussage von Kreye ${ }^{315}$, dass sich die Umweltgeschichte vornehmlich mit materiellen Werten (Veränderungen der physikalischen Umwelt) befasse. Auch die Feststellung von Uekötter ${ }^{316}$, dass es unter den Forschern einen Konsens hinsichtlich der anthropozentrisch begründeten Fragestellungen der Umweltgeschichte zu geben scheint, wird durch die durchgängig anthropozentrische Ausrichtung der dominierenden Werte bestätigt.

Was angesichts der grundsätzlichen Priorität von Luft und Wasser für den Menschen verwundert, ist die geringe Anzahl an Fundstellen zu diesen Werten bzw. die insgesamt niedrige Platzierung. So erreicht der Wert Luft lediglich Rang 21, der Wert Wasser Rang 8. Andererseits belegt der Wert Gesundheit sowohl insgesamt als auch nach Ländern Platz 1 oder 2. Des Weiteren bedeutet die Platzierung des Wertes Leben, der mit Rang 5 (nur) eine mittlere Platzierung erreicht, nicht unbedingt dass Leben nur eine mittlere Priorität besitzt, denn ein Umweltschaden muss nicht zu Todesopfern führen. Für diese Hypothese spricht

\footnotetext{
${ }^{313}$ Vgl. Rolf Peter Sieferle, Die Grenzen der Umweltgeschichte, in: GAIA 2 (1993) 1, S. 8-21, hier S. 9.

314 Vgl. Joachim Radkau, Unbekannte Umwelt: von der altklugen zur neugierigen Umweltgeschichte, in: Praxis Geschichte (4), Braunschweig 1997, S. 4-10, hier S. 6.

${ }^{315} \mathrm{Vgl}$. Fußnote 96, S. 23.

${ }^{316}$ Vgl. Fußnote 232, S. 52.
} 
die hohe Platzierung des Wertes Gesundheit. Das heißt, dass die in den Texten behandelten Umweltschäden wahrscheinlich häufiger zu gesundheitlichen Beeinträchtigungen führten als zum Tod.

Auch hinsichtlich des erstplatzierten Wertes Ökonomie sind einige Punkte zu diskutieren. Zunächst ist Ökonomie als bestimmender Wert kritisch zu sehen, denn ein Umweltschaden provoziert geradezu eine ökonomische Bewertung um die Auswirkungen greifbarer zu machen und eine vergleichbare Größenordnung zu schaffen. Auch die Verfügbarkeit von Daten könnte hier eine Rolle spielen. Dennoch sagt die starke Präsenz in den umweltgeschichtlichen Texten etwas über die Prioritäten bei der Beschreibung eines Umweltschadens aus, insbesondere vor dem Hintergrund, dass immaterielle Werte vergleichsweise selten vertreten sind und unter den ersten acht Werten nicht vorkommen. Zudem ist zu fragen, warum in der umweltgeschichtlichen Forschung ökonomische Aspekte von Umweltschäden so stark vertreten sind. Eine fundierte Bewertung eines Umweltschadens mit allen zugehörigen Kosten ist eine komplexe Aufgabe, insbesondere wenn alle Effekte internalisiert werden sollen, d. h. auch solche, für die es keine rechtliche Grundlage zur Übernahme der Kosten gibt. Die Fundstellen in den Texten deuten jedoch darauf hin, dass die ökonomische Bewertung je nach Datenlage eher als ergänzendes Element gesehen wird. Umweltgeschichte befasst sich mit den Wechselwirkungen zwischen Mensch und Umwelt. Dies erfordert das Zusammenwirken verschiedenster Disziplinen. Weshalb aber wurde als am häufigsten vertretener Wert gerade die Ökonomie ermittelt? Eigentlich sollten die ökologischen Aspekte eine höhere Priorität haben als die ökonomischen, da sie direkt die Lebensgrundlage der Menschen betreffen.

Welche Schlussfolgerungen lassen sich nun aus den gewonnenen Erkenntnissen ableiten? „Umweltgeschichte zeigt den Wandel von Bewertungen. “317 Winiwarter spricht hier vermutlich die Bewertungen durch die Gesellschaft an. Aber auch die Bewertungen durch die Forschenden ändern sich. Wenngleich sich die vorliegende Arbeit nicht mit Wertewandel befasst, so ist doch festzustellen, dass mit der hier verwendeten Methode die einem Text zugrundeliegende Werte aufgezeigt werden können. Denn nicht nur die Tatsache, dass sich Bewertungen gewandelt haben bzw. das Wie ist hierbei von Interesse, sondern auch - und das ist vielleicht sogar von größerer Bedeutung - auf welcher Basis diese Wandlung stattgefunden hat. Erst die Kenntnis der zugrunde liegenden Werte führt zu Erkenntnissen in Bezug auf die Gründe von Bewertungsänderungen. Dies könnte für die Umweltgeschichte insofern hilfreich sein, als dass sich auch die Bewertungen der Forscher selbst wandeln. Durch das Sichtbarmachen dieser Prozesse können sich somit auch neue Erkenntnisse zur Entwicklung der Umweltgeschichte ergeben.

\footnotetext{
${ }^{317}$ Verena Winiwarter/Hans-Rudolf Bork, Umweltgeschichte: ein Plädoyer für Rücksicht und Weitsicht: [Vortrag im Großen Sendesaal des ORF-RadioKulturhauses am 20. März 2014], Wien, 2014, S. 28.
} 
Weiterhin hat die Textanalyse gezeigt, dass in umweltgeschichtlichen Texten, die den Begriff Umweltschaden enthalten anthropozentrische, nutzungsabhängige Werte vorherrschen. Das ist zunächst weder als gut noch als schlecht zu beurteilen, jedoch könnte ein bewusster Perspektivwechsel hier vielleicht neue Erkenntnisse zutage fördern. Es bedeutet nicht, dass der anthropozentrische Ansatz der Umweltgeschichte grundsätzlich in Frage gestellt wird, denn laut Radkau - und hier ist ihm zuzustimmen - ergibt eine nicht-anthropozentrische Umweltgeschichte keinen Sinn. ${ }^{318}$ Aber die Erkenntnis, dass und wie stark anthropozentrische Werte die Bearbeitung umweltgeschichtlicher Themen tatsächlich beeinflussen, sollte zum Nachdenken darüber anregen, inwieweit dies bewusst geschieht und ob bei Zugrundlegung anderer Wertvorstellungen die Bearbeitung einer Fragestellung anders verlaufen würde bzw. wie stark sich das Ergebnis infolgedessen verändern würde.

${ }^{318}$ Vgl. Joachim Radkau, Unbekannte Umwelt: von der altklugen zur neugierigen Umweltgeschichte, in: Praxis Geschichte (4), Braunschweig 1997, S. 4-10, hier S. 6. 


\section{Literaturverzeichnis}

Abelshauser, Werner (Hrsg.), Umweltgeschichte: Umweltverträgliches Wirtschaften in historischer Perspektive ; acht Beiträge, Göttingen, 1994.

Albert, Hans/Tropisch, Ernst (Hrsg.), Werturteilsstreit, Darmstadt, 1979.

Allemeyer, Marie Luisa/Behrens, Katharina/Mersch, Katharina Ulrike (Hrsg.), Eule oder Nachtigall?: Tendenzen und Perspektiven kulturwissenschaftlicher Werteforschung, Göttingen, 2007.

Barkmann, Jan/Marggraf, Rainer, Ökologische Schäden durch Vernachlässigung des Vorsorgeprinzips im nachhaltigen Landschaftsmanagement - eine umweltökonomische Perspektive, in: Thomas Potthast (Hrsg.), Ökologische Schäden. Begriffliche, methodologische und ethische Aspekte 2004, S. 57-76.

Bayerl, Günter (Hrsg.), Umweltgeschichte - Methoden, Themen, Potentiale, Münster [u.a.], 1996.

Behrens, Hermann/Auster, Regine (Hrsg.), Umweltgeschichte Wissenschaft und Praxis: Umweltgeschichte und Umweltzukunft II, Marburg, 1994.

Bird, E. A. R., The social construction of Nature: Theoretical approaches to the history of Environmental Problems, in: Environmental Review (1987), S. 255-264.

Borries, Bodo von, Didaktische Möglichkeiten und Grenzen der Umweltgeschichte, in: Günter Bayerl (Hrsg.), Umweltgeschichte - Methoden, Themen, Potentiale, Münster [u.a.] 1996, S. 309-326.

Brandt, Ahasver von, Werkzeug des Historikers: Eine Einführung in die historischen Hilfswissenschaften, Stuttgart, 2003.

Breckling, Broder/Potthast, Thomas, Der ökologische Schadensbegriff - eine Einführung, in: Thomas Potthast (Hrsg.), Ökologische Schäden. Begriffliche, methodologische und ethische Aspekte 2004, S. 1-16.

Brüggemeier, Franz-Josef, Umweltgeschichte - Erfahrungen, Ergebnisse, Erwartungen, in: Archiv für Sozialgeschichte (2003), S. 1-18.

Bueb, Bernhard et al (Hrsg.), Alte Werte - Neue Werte: Schlaglichter des Wertewandels, Göttingen, 2008.

Bundesnaturschutzgesetz: BNatSchG, 07.08.2013.

Carrier, Martin, Wissenschaftstheorie zur Einführung, Hamburg, 2008.

Castelli Gattinara, Enrico, Wahrheit, in: Anne Kwaschik/Mario Wimmer (Hrsg.), Von der Arbeit des Historikers. Ein Wörterbuch zu Theorie und Praxis der Geschichtswissenschaft, Bielefeld 2010, S. 215-218.

Castree, Noel/Braun, Bruce (Hrsg.), Social nature: Theory, practice, and politics, Malden, Mass., 2001.

Clement, Cathie (Hrsg.), Ethics and the practice of history, Perth, W.A., 2010.

Cornelißen, Christoph/Budde, Gunilla-Friederike, Geschichtswissenschaften: Eine Einführung, Frankfurt am Main, 2004.

Crowards, Tom, Nonuse Values and the Environment: Economic and Ethical Motivations, in: Environmental Values (1997), S. 143-167.

Endres, Alfred/Holm-Müller, Karin, Die Bewertung von Umweltschäden: Theorie und Praxis sozioökonomischer Verfahren, Stuttgart [u.a.], 1998.

Engels, Jens Ivo, Umweltgeschichte als Zeitgeschichte, in: Aus Politik und Zeitgeschichte (2006), S. 32-38. 
Faber, Karl-Georg, Theorie der Geschichtswissenschaft, München, 1982.

Feix, Nereu, Werturteil, Politik und Wirtschaft, Göttingen, 1978.

Flick, Uwe/Kardorff, Ernst von/Steinke, Ines (Hrsg.), Qualitative Forschung: Ein Handbuch, Reinbek bei Hamburg, 2009.

Flick, Uwe/Kardorff, Ernst von/Steinke, Ines, Was ist qualitative Forschung? Einleitung und Überblick, in: Uwe Flick/Ernst von Kardorff/Ines Steinke (Hrsg.), Qualitative Forschung. Ein Handbuch, Reinbek bei Hamburg 2009, S. 13-29.

Fuchsloch, Herrmann, Methodenfragen der Umweltgeschichte, in: Günter Bayerl (Hrsg.), Umweltgeschichte - Methoden, Themen, Potentiale, Münster [u.a.] 1996, S. 1-12.

Gadamer, Hans-Georg, Wahrheit und Methode: Grundzüge einer philosophischen Hermeneutik, Tübingen, 1960.

Goertz, Hans-Jürgen, Umgang mit Geschichte: Eine Einführung in die Geschichtstheorie, Reinbek bei Hamburg, 1995.

Grewe, Bernd-Stefan, Umweltgeschichte unterrichten: Für eine kritische Auseinandersetzung mit umwelthistorischen Denkmustern, in: Britta Wehen-Behrens/Dietmar von Reeken/Indre Döpke (Hrsg.), Umweltgeschichte lehren und lernen. Keine Katastrophe!, Schwalbach am Taunus 2015, S. 84-100.

Grove, Richard H., Die Anfänge des Umweltbewußtseins, in: Ernst Ulrich von Weizsäcker (Hrsg.), Mensch, Umwelt, Wirtschaft, Heidelberg [u.a.] 1995, S. 18-23.

Günther, Armin, Technologische Risiken zwischen Wahrnehmung, Konstruktion und Reflexion, in: Martin Stengel (Hrsg.), Umweltökonomie. Eine interdisziplinäre Einführung, München 1997, S. 111-135.

Hahn, Sylvia/Reith, Reinhold, Einleitung, in: Sylvia Hahn/Reinhold Reith (Hrsg.), UmweltGeschichte. Arbeitsfelder - Forschungsansätze - Perspektiven, Wien 2001, S. 7-12.

Hahn, Sylvia/Reith, Reinhold (Hrsg.), Umwelt-Geschichte: Arbeitsfelder - Forschungsansätze - Perspektiven, Wien, 2001.

Hauhs, Michael/Lange, Holger, Was ist ein ökologischer Schaden? Ein Ansatz für die Bestimmung virtueller Standards, in: Thomas Potthast (Hrsg.), Ökologische Schäden. Begriffliche, methodologische und ethische Aspekte 2004, S. 25-50.

Hays, Samuel P., Explorations in environmental history: Essays, Pittsburgh, Pa., 1998.

Hedinger, Hans-Walter, Subjektivität und Geschichtswissenschaft: Grundzüge einer Historik. Univ., Diss.--Hamburg, 1967, Berlin, 1969.

Helbling, Jürg, Ökologie und Politik in nicht-staatlichen Gesellschaften oder: Wie steht es mit der Naturverbundenheit sogenannter Naturvölker, in: Kölner Zeitschrift für Soziologie und Sozialpsychologie (1992), S. 203-225.

Helbling, Jürg, Der Einfluss religiöser Vorstellungen, Normen und Rituale auf die Ressourcennutzung in einfachen Gesellschaften am Beispiel der Cree und der Maring, in: Rolf Peter Sieferle/Helga Breuninger (Hrsg.), Natur-Bilder. Wahrnehmungen von Natur und Umwelt in der Geschichte, Frankfurt/Main 1999, S. 19-42.

Herbst, Ludolf, Komplexität und Chaos: Grundzüge einer Theorie der Geschichte, München, 2004.

Herrman, Bernd, Umweltgeschichte als Integration von Natur- und Kulturwissenschaften, in: Günter Bayerl (Hrsg.), Umweltgeschichte - Methoden, Themen, Potentiale, Münster [u.a.] 1996, S. 21-30.

Herz, Thomas, Der Wandel der Wertvorstellungen in westlichen Industriegesellschaften, in: Kölner Zeitschrift für Soziologie und Sozialpsychologie (1979), S. 282-302. 
Hesse, Heidrun, Umweltschäden und ökologisches Wissen - kleine Zwischenbetrachtung aus philosophischer Sicht, in: Thomas Potthast (Hrsg.), Ökologische Schäden. Begriffliche, methodologische und ethische Aspekte 2004, S. 51-56.

Hettling, Manfred (Hrsg.), Was ist Gesellschaftsgeschichte?: Positionen Themen Analysen ; [Hans-Ulrich Wehler zum 60. Geburtstag], München, 1991.

Howell, Martha, Werkstatt des Historikers: Eine Einführung in die historischen Methoden, Köln, 2004.

Hughes, J. Donald, What is environmental history?, Cambridge, 2006.

Jaeger, Friedrich/Straub, Jürgen (Hrsg.), Handbuch der Kulturwissenschaften: Band 3. Paradigmen und Disziplinen, 2004.

Jonas, Klaus/Stroebe, Wolfgang/Hewstone, Miles/Lebherz, Carmen/Reiss, Matthias, Sozialpsychologie: Eine Einführung, Heidelberg, 2007.

Jordan, Stefan, Theorien und Methoden der Geschichtswissenschaft, Paderborn, 2009.

Kalt, Monika, Umweltgeschichte - Revisited, in: Traverse: Zeitschrift für Geschichte (1997), S. 13-30.

Keller, Reiner, Handbuch Sozialwissenschaftliche Diskursanalyse, Wiesbaden, 2008.

Kluckhohn, Clyde, Values and Value-Orientations in the Theory of Action: an Exploration in Definition and Classification, in: Talcott Parsons/Edward Shils/Edward C. Tolman (Hrsg.), Toward a general theory of action, New York, NY 1962.

Kolmer, Lothar, Geschichtstheorien, Paderborn, 2008.

Krause/Marggraf/Meyer, Kann die Umweltökonomie den intrinsischen Wert berücksichtigen?, in: Zeitschrift für Umweltpolitik und Umweltrecht (2008), S. 293-313.

Kreye, Lars/Schwarzer, Markus, Verständnis und Institutionalisierung der Umweltgeschichte, in: Patrick Masius/Ole Sparenberg/Jana Sprenger (Hrsg.), Umweltgeschicht und Umweltzukunft. Zur gesellschaftlichen Relevanz einer jungen Disziplin, Göttingen 2009, S. 3-11.

Kruse, Lenelis/Graumann, C. -F/Lantermann, E. -D, Ökologische Psychologie: Ein Handbuch in Schlüsselbegriffen, München, 1990.

Kuckartz, Udo, Trends im Umweltbewusstsein: Umweltgerechtigkeit, Lebensqualität und persönliches Engagement, Wiesbaden, 2006.

Kwaschik, Anne/Wimmer, Mario (Hrsg.), Von der Arbeit des Historikers: Ein Wörterbuch zu Theorie und Praxis der Geschichtswissenschaft, Bielefeld, 2010.

Lamnek, Siegfried, Qualitative Sozialforschung: Lehrbuch, Weinheim, 2005.

Lehmkuhl, Ursula, Historicizing Nature: Time and Space in German and American Environmental History, in: Ursula Lehmkuhl/Herrmann Wellenreuther (Hrsg.), Historians and nature. Comparative approaches to environmental history, Oxford [u.a.] 2007, S. 17-44.

Lehmkuhl, Ursula, Introduction, in: Ursula Lehmkuhl/Herrmann Wellenreuther (Hrsg.), Historians and nature. Comparative approaches to environmental history, Oxford [u.a.] 2007, S. 1-5.

Lehmkuhl, Ursula/Wellenreuther, Herrmann (Hrsg.), Historians and nature: Comparative approaches to environmental history, Oxford [u.a.], 2007.

Linneweber, Volker/Kals, Elisabeth (Hrsg.), Umweltgerechtes Handeln: Barrieren und Brücken, Berlin, 1999.

Lisbach, Bertrand, Werte und Umweltwahrnehmung: Der Einfluß von Werten auf die Wahrnehmung der natürlichen Umwelt, Marburg, 1999. 
Littig, Beate, Die Bedeutung von Umweltbewußtsein im Alltag: Oder: was tun wir eigentlich, wenn wir umweltbewußt sind?, Frankfurt am Main, 1995.

Masius, Patrick/Sparenberg, Ole/Sprenger, Jana (Hrsg.), Umweltgeschicht und Umweltzukunft. Zur gesellschaftlichen Relevanz einer jungen Disziplin, Göttingen, 2009.

Mayring, Philipp, Einführung in die qualitative Sozialforschung: Eine Anleitung zu qualitativem Denken, Weinheim, 2002.

Mayring, Philipp, Qualitative Inhaltsanalyse: Grundlagen und Techniken, Weinheim [u.a.], 2008.

Mayring, Philipp, Qualitative Inhaltsanalyse, in: Uwe Flick/Ernst von Kardorff/Ines Steinke (Hrsg.), Qualitative Forschung. Ein Handbuch, Reinbek bei Hamburg 2009, S. 468474.

McNeill, J. R., Observations on the nature and culture of environmental history, in: History and Theory (2003), S. 5-43.

Meinicke, Klaus-Peter, Umweltgeschichte au ingenieurwissenschaftlicher Perspektive, in: Günter Bayerl (Hrsg.), Umweltgeschichte - Methoden, Themen, Potentiale, Münster [u.a.] 1996, S. 285-303.

Merkens, Hans, Auswahlverfahren, Sampling, Fallkonstruktion, in: Uwe Flick/Ernst von Kardorff/Ines Steinke (Hrsg.), Qualitative Forschung. Ein Handbuch, Reinbek bei Hamburg 2009, S. 286-298.

Mersch, Katharina Ulrike, Überlegungen zum Wert historischer Werteforschung, in: Marie Luisa Allemeyer/Katharina Behrens/Katharina Ulrike Mersch (Hrsg.), Eule oder Nachtigall? Tendenzen und Perspektiven kulturwissenschaftlicher Werteforschung, Göttingen 2007, S. 9-34.

Merten, Klaus, Inhaltsanalyse: Einführung in Theorie, Methode und Praxis, Opladen, 1995.

Miller, Shawn William, An environmental history of Latin America, New York, 2007.

Mutschler, Hans-Dieter, Gibt es Werte in der Natur?, in: Marie Luisa Allemeyer/Katharina Behrens/Katharina Ulrike Mersch (Hrsg.), Eule oder Nachtigall? Tendenzen und Perspektiven kulturwissenschaftlicher Werteforschung, Göttingen 2007, S. 221-239.

Opp, Karl-Dieter, Methodologie der Sozialwissenschaften: Einführung in Probleme ihrer Theoriebildung und praktischen Anwendung, Wiesbaden, 2002.

Ott, Konrad, Umweltethik zur Einführung, Hamburg, 2010.

Ott, Konrad/Gorke, Martin (Hrsg.), Spektrum der Umweltethik, Marburg, 2000.

Paravicini, Werner, Die Wahrheit der Historiker, München, 2010.

Parsons, Talcott/Shils, Edward/Tolman, Edward C. (Hrsg.), Toward a general theory of action, New York, NY, 1962.

Paul Leidinger, Von der historischen Umweltforschung zur Historischen Ökologie. Ein Literaturbericht (Teil 1), in: Hermann Behrens/Regine Auster (Hrsg.), Umweltgeschichte Wissenschaft und Praxis. Umweltgeschichte und Umweltzukunft II, Marburg 1994, S. 11-39.

Pfeiffer, Till/Manz, Sylvia/Nerb, Josef, Wer den Schaden macht, hat auch das Wissen: Kohärenzeffekte der kognitiven und emotionalen Bewertung von Umweltschadensfällen, in: Zeitschrift für Psychologie (2005), S. 44-58.

Poser, Hans, Wissenschaftstheorie: Eine philosophische Einführung, Stuttgart, 2001. 
Potthast, Thomas, Wo sich Biologie, Ethik und Naturphilosophie treffen (müssen): Epistemologische und moralphilosophische Aspekte der Umweltethik, in: Konrad Ott/Martin Gorke (Hrsg.), Spektrum der Umweltethik, Marburg 2000, S. 101-141.

Potthast, Thomas (Hrsg.), Ökologische Schäden: Begriffliche, methodologische und ethische Aspekte, 2004.

Potthast, Thomas, Ökologische Schäden - eine Synopse begrifflicher, methodologischer und ethischer Aspekte, in: Thomas Potthast (Hrsg.), Ökologische Schäden. Begriffliche, methodologische und ethische Aspekte 2004, S. 189-209.

Proctor, James D., Solid Rock and Shifting Sands: The Moral Paradox of Saving a Socially Constructed Nature, in: Noel Castree/Bruce Braun (Hrsg.), Social nature. Theory, practice, and politics, Malden, Mass. 2001, S. 225-239.

Radkau, Joachim, Unausdiskutiertes in der Umweltgeschichte, in: Manfred Hettling (Hrsg.), Was ist Gesellschaftsgeschichte? Positionen Themen Analysen ; [Hans-Ulrich Wehler zum 60. Geburtstag], München 1991, S. 44-57.

Radkau, Joachim, Was ist Umweltgeschichte?, in: Werner Abelshauser (Hrsg.), Umweltgeschichte. Umweltverträgliches Wirtschaften in historischer Perspektive ; acht Beiträge, Göttingen 1994, S. 11-28.

Radkau, Joachim, Unbekannte Umwelt: von der altklugen zur neugierigen Umweltgeschichte, in: Praxis Geschichte (4), Braunschweig 1997, S. 4-10.

Radkau, Joachim, Erfahrungen und Grübeleien beim Schreiben an einer WeltUmweltgeschichte, in: Universität Bielefeld, Informations- und Pressestelle (Hrsg.), Forschung an der Universität Bielefeld, Bielefeld 2000, S. 42-49.

Radkau, Joachim, Natur und Macht: Eine Weltgeschichte der Umwelt, München, 2000.

Radkau, Joachim, Nachdenken über Umweltgeschichte, in: Wolfram Siemann (Hrsg.), Umweltgeschichte. Themen und Perspektiven, München 2003, S. 165-186.

Reusswig, Fritz, Umweltgerechtes Handeln in verschiedenen Lebensstil-Kontexten, in: Volker Linneweber/Elisabeth Kals (Hrsg.), Umweltgerechtes Handeln. Barrieren und Brücken, Berlin 1999, S. 49-67.

Rheinberger, Hans-Jörg, Historische Epistemologie, in: Anne Kwaschik/Mario Wimmer (Hrsg.), Von der Arbeit des Historikers. Ein Wörterbuch zu Theorie und Praxis der Geschichtswissenschaft, Bielefeld 2010, S. 103-105.

Rickert, Heinrich/Vollhardt, Friedrich, Kulturwissenschaft und Naturwissenschaft, Stuttgart, 1986.

Rödder, Andreas, Werte und Wertewandel: Historisch-politische Perspektiven, in: Bernhard et al Bueb (Hrsg.), Alte Werte - Neue Werte. Schlaglichter des Wertewandels, Göttingen 2008, S. 9-25.

Rosenthal, Gabriele, Interpretative Sozialforschung: Eine Einführung, Weinheim, 2005.

Rossi, Pietro (Hrsg.), Theorie der modernen Geschichtsschreibung, Frankfurt am Main, 1987.

Schaff, Adam, Geschichte und Wahrheit, Wien, 1970.

Schenk, Gerrit Jasper, Der Mensch zwischen Natur und Kultur. Auf der Suche nach der Umweltgeschichtsschreibung in der deutschsprachigen Mediävistik - eine Skizze, in: François Duceppe-Lamarre (Hrsg.), Umwelt und Herrschaft in der Geschichte, München 2008, S. 27-51.

Schmidt, Paul F., Ethische Normen in der wissenschaftlichen Methode, in: Hans Albert/Ernst Tropisch (Hrsg.), Werturteilsstreit, Darmstadt 1979, S. 353-364. 
Schmitt, Jean-Claude, Welche Geschichte der Werte?, in: Barbara StollbergRilinger/Thomas Weller (Hrsg.), Wertekonflikte - Deutungskonflikte. Internationales Kolloquium des Sonderforschungsbereichs 496 an der Westfälischen WilhelmsUniversität Münster 19. - 20. Mai 2005, Münster 2007, S. 21-35.

Schmoelz, Franz-Martin/Weingartner, Paul (Hrsg.), Werte in den Wissenschaften: Festschrift zum 30jährigen Bestehen des Internationalen Forschungszentrums in Salzburg, Innsbruck, 1991.

Schweidler, Walter (Hrsg.), Werte im 21. Jahrhundert, Baden-Baden, 2001.

Sieferle, Rolf Peter, Die Grenzen der Umweltgeschichte, in: GAIA (1993), S. 8-21.

Sieferle, Rolf Peter, Einleitung: Naturerfahrung und Naturkonstruktion, in: Rolf Peter Sieferle/Helga Breuninger (Hrsg.), Natur-Bilder. Wahrnehmungen von Natur und Umwelt in der Geschichte, Frankfurt/Main 1999, S. 9-18.

Sieferle, Rolf Peter/Breuninger, Helga (Hrsg.), Natur-Bilder: Wahrnehmungen von Natur und Umwelt in der Geschichte, Frankfurt/Main, 1999.

Siemann, Wolfram (Hrsg.), Umweltgeschichte: Themen und Perspektiven, München, 2003.

Skorupinski, Barbara, Gentechnik und ökologische Schäden als Gegenstand von risikoforschung und partizipativer Technikfolgenabschätzung - Stand und Perspektiven, in: Thomas Potthast (Hrsg.), Ökologische Schäden. Begriffliche, methodologische und ethische Aspekte 2004, S. 169-188.

Smieszek, Timo, Unsicherheit, Werthaltungen und Handlungsblockaden, in: GAIA (2006), S. 251-254.

Soeffner/Raab, Kulturwissenschaftliche Methoden und Ansätze in den Disziplinen, in: Friedrich Jaeger/Jürgen Straub (Hrsg.), Handbuch der Kulturwissenschaften. Band 3. Paradigmen und Disziplinen 2004, S. 552-567.

Steinke, Ines, Gütekriterien qualitativer Forschung, in: Uwe Flick/Ernst von Kardorff/Ines Steinke (Hrsg.), Qualitative Forschung. Ein Handbuch, Reinbek bei Hamburg 2009, S. 319-331.

Stengel, Martin (Hrsg.), Umweltökonomie: Eine interdisziplinäre Einführung, München, 1997.

Stern, Alfred, Geschichtsphilosophie und Wertproblem, München [u.a.], 1967.

Stollberg-Rilinger, Barbara, Die Historiker und die Werte, in: Marie Luisa Allemeyer/Katharina Behrens/Katharina Ulrike Mersch (Hrsg.), Eule oder Nachtigall? Tendenzen und Perspektiven kulturwissenschaftlicher Werteforschung, Göttingen 2007, S. 35-48.

Stollberg-Rilinger, Barbara, Einleitung, in: Barbara Stollberg-Rilinger/Thomas Weller (Hrsg.), Wertekonflikte - Deutungskonflikte. Internationales Kolloquium des Sonderforschungsbereichs 496 an der Westfälischen Wilhelms-Universität Münster 19. - 20. Mai 2005, Münster 2007, S. 9-20.

Stollberg-Rilinger, Barbara/Weller, Thomas (Hrsg.), Wertekonflikte - Deutungskonflikte: Internationales Kolloquium des Sonderforschungsbereichs 496 an der Westfälischen Wilhelms-Universität Münster 19. - 20. Mai 2005, Münster, 2007.

Strack, M./Gennerich, C./Hopf, N., Warum Werte?, in: Erich H. Witte (Hrsg.), Sozialpsychologie und Werte, s.I. 2008, S. 91-131.

Strauss, Leo, Die Unterscheidung zwischen Tatsachen und Werten, in: Hans Albert/Ernst Tropisch (Hrsg.), Werturteilsstreit, Darmstadt 1979, S. 73-91.

Tapper, Alan, Is there an Ethics for Historians?, in: Cathie Clement (Hrsg.), Ethics and the practice of history, Perth, W.A. 2010, S. 16-36.

Thukydides/Vretska, Helmuth, Der Peloponnesische Krieg, Stuttgart, 2014. 
Topolski, Jerzy, Die Wissenschaftlichkeit der Geschichtsschreibung und ihre Grenzen, in: Pietro Rossi (Hrsg.), Theorie der modernen Geschichtsschreibung, Frankfurt am Main 1987, S. 191-217.

Troitzsch, Ulrich, Historische Umweltforschung: Einleitende Bemerkungen über Forschungsstand und Forschungsaufgaben, in: Technikgeschichte (1981), S. 177-190.

Uekötter, Frank, Umweltgeschichte im 19. und 20. Jahrhundert, München, 2007.

Umweltschadensgesetz: USchadG, 20.04.2013.

Universität Bielefeld, Informations- und Pressestelle (Hrsg.), Forschung an der Universität Bielefeld, Bielefeld, 2000.

Urban, D., Was ist Umweltbewußtsein?, in: Zeitschrift für Soziologie (1986), S. 363-377.

Voland, Eckart, Welche Werte? Ethik, Anthropologie und Naturschutz, in: Philosophia naturalis (2000), S. 131-152.

Weber, Max, Wissenschaft als Beruf, 2006.

Weingartner, Paul, Werte in den Wissenschaften, in: Franz-Martin Schmoelz/Paul Weingartner (Hrsg.), Werte in den Wissenschaften. Festschrift zum 30jährigen Bestehen des Internationalen Forschungszentrums in Salzburg, Innsbruck 1991, S. $11-41$.

Weizsäcker, Ernst Ulrich von (Hrsg.), Mensch, Umwelt, Wirtschaft, Heidelberg [u.a.], 1995.

Winiwarter, Verena/Knoll, Martin, Umweltgeschichte: Eine Einführung, Köln [u.a.], 2007.

Winiwarter, Verena/Bork, Hans-Rudolf, Umweltgeschichte: ein Plädoyer für Rücksicht und Weitsicht: [Vortrag im Großen Sendesaal des ORF-RadioKulturhauses am 20. März 2014], Wien, 2014.

Witte, Erich H. (Hrsg.), Sozialpsychologie und Werte, s.l., 2008.

Zirnstein, Gottfried (Hrsg.), Ökologie und Umwelt in der Geschichte, Marburg, 1994. 


\section{Anhang}

\section{A Hochschulen in Deutschland mit Geschichte als Studiengang}

\begin{tabular}{|c|c|c|c|}
\hline Bezeichnung & Ort & $\begin{array}{l}\text { Zugriff } \\
\text { am }\end{array}$ & URL des jeweiligen Geschichtsbereichs \\
\hline RWTH & Aachen & 15.07 .14 & $\begin{array}{l}\text { http://www.histinst.rwth- } \\
\text { aachen.de/aw/cms/HISTINST/startseite/ vgw/home/?lang=de }\end{array}$ \\
\hline Universität & Augsburg & 16.07 .14 & http://www.philhist.uni-augsburg.de/de/lehrstuehle/geschichte/Lehrstuehle/ \\
\hline $\begin{array}{l}\text { Otto-Friedrich- } \\
\text { Universität }\end{array}$ & Bamberg & 16.07 .14 & http://www.uni-bamberg.de/hist/ \\
\hline $\begin{array}{l}\text { Freie } \\
\text { Universität }\end{array}$ & Berlin & 16.07 .14 & $\begin{array}{l}\text { http://www.fu-berlin.de/einrichtungen/fachbereiche/fb/gesch- } \\
\text { kultur/geschichte/index.html }\end{array}$ \\
\hline $\begin{array}{l}\text { Humboldt- } \\
\text { Universität }\end{array}$ & Berlin & 16.07.14 & http://www.geschichte.hu-berlin.de/bereiche-und-lehrstuehle \\
\hline $\begin{array}{l}\text { Ruhr- } \\
\text { Universität }\end{array}$ & Bochum & 16.07 .14 & $\begin{array}{l}\text { http://www.ruhr-uni-bochum.de/tug/ } \\
\text { http://www.ruhr-uni-bochum.de/umweltgeschichte/ }\end{array}$ \\
\hline $\begin{array}{l}\text { Rheinische } \\
\text { Friedrich- } \\
\text { Wilhelms } \\
\text { Universität }\end{array}$ & Bonn & 17.07.14 & http://www.igw.uni-bonn.de/institut/organisation-abteilungen \\
\hline $\begin{array}{l}\text { TU Carolo- } \\
\text { Wilhelmina }\end{array}$ & Braunschweig & 17.07.14 & http://www.historisches-seminar-braunschweig.de/ \\
\hline Universität & Bremen & 17.07.14 & http://www.geschichte.uni-bremen.de/?page_id=4 \\
\hline TU & Darmstadt & 17.07 .14 & http://www.geschichte.tu-darmstadt.de/index.php?id=3125 \\
\hline TU & Dresden & 17.07.14 & $\underline{\underline{\text { http://tu- }}}$ dresden.de/die_tu_dresden/fakultaeten/philosophische_fakultaet/inst/geschichte \\
\hline $\begin{array}{l}\text { Heinrich- } \\
\text { Heine- } \\
\text { Universität }\end{array}$ & Düsseldorf & 17.07 .14 & http://www.phil-fak.uni-duesseldorf.de/geschichte/ \\
\hline $\begin{array}{l}\text { Katholische } \\
\text { Universität }\end{array}$ & $\begin{array}{l}\text { Eichstätt- } \\
\text { Ingolstadt }\end{array}$ & 17.07 .14 & http://www.ku.de/ggf/ \\
\hline $\begin{array}{l}\text { Friedrich- } \\
\text { Alexander- } \\
\text { Universität }\end{array}$ & $\begin{array}{l}\text { Erlangen- } \\
\text { Nürnberg }\end{array}$ & 17.07 .14 & $\begin{array}{l}\text { http://www.osteuropa.geschichte.uni-erlangen.de/cms/mitarbeiter/prof.-dr.-julia- } \\
\text { obertreis/forschungsschwerpunkte.php }\end{array}$ \\
\hline Universität & $\begin{array}{l}\text { Duisburg- } \\
\text { Essen }\end{array}$ & 17.07.14 & https://www.uni-due.de/geschichte/personal.php?SHOW=abt-list \\
\hline $\begin{array}{l}\text { Goethe- } \\
\text { Universität }\end{array}$ & Frankfurt & 17.07.14 & http://www.geschichte.uni-frankfurt.de/43061944/ng \\
\hline $\begin{array}{l}\text { Albert- } \\
\text { Ludwigs- } \\
\text { Universität }\end{array}$ & Freiburg & 17.07 .14 & http://www.wsu.geschichte.uni-freiburg.de/ \\
\hline $\begin{array}{l}\text { Justus-Liebig- } \\
\text { Universität }\end{array}$ & Gießen & 17.07 .14 & $\begin{array}{l}\text { http://www.uni- } \\
\text { giessen.de/cms/fbz/fb04/institute/geschichte/osteuropa/HaDi/Ost/A.\%20Kouida/K } \\
\text { ouida_Diss }\end{array}$ \\
\hline $\begin{array}{l}\text { Georg-August- } \\
\text { Universität }\end{array}$ & Göttingen & 17.07 .14 & http://www.uni-goettingen.de/de/umweltgeschichte/215059.html \\
\hline $\begin{array}{l}\text { Ernst-Moritz- } \\
\text { Arndt- } \\
\text { Universität }\end{array}$ & Greifswald & 17.07 .14 & http://www.phil.uni-greifswald.de/bereich2/histin.html \\
\hline $\begin{array}{l}\text { Martin-Luther- } \\
\text { Universität }\end{array}$ & $\begin{array}{l}\text { Halle- } \\
\text { Wittenberg }\end{array}$ & 17.07 .14 & http://archaeology.altertum.uni-halle.de/mitarbeiter/nenninger/ \\
\hline Universität & Hamburg & 17.07.14 & $\begin{array}{l}\text { http://www1.geschichte.uni-hamburg.de/de/arbeitsbereiche/deutsche- } \\
\text { geschichte/schwerpunkt-sozial-und-wirtschaftsgeschichte/arbeitsstelle-fuer- } \\
\text { sozialgeschichte-der-umwelt-und-technik.html }\end{array}$ \\
\hline $\begin{array}{l}\text { Gottfried } \\
\text { Wilhelm } \\
\text { Leibniz } \\
\text { Universität }\end{array}$ & Hannover & 17.07.14 & http://www.hist.uni-hannover.de/fachgebiete hist.html \\
\hline $\begin{array}{l}\text { Ruprecht- } \\
\text { Karls- } \\
\text { Universität }\end{array}$ & Heidelberg & 17.07 .14 & $\begin{array}{l}\frac{\text { http://www.uni-heidelberg.de/fakultaeten/philosophie/zegk/histsem/index.html }}{\text { http://www.asia-europe.uni-heidelberg.de/de/forschung/a-regierungskunst- }} \\
\underline{\text { verwaltung/a6/publications-of-project-members.html }}\end{array}$ \\
\hline
\end{tabular}




\begin{tabular}{|c|c|c|c|}
\hline Bezeichnung & Ort & $\begin{array}{l}\text { Zugriff } \\
\text { am }\end{array}$ & URL des jeweiligen Geschichtsbereichs \\
\hline $\begin{array}{l}\text { Friedrich- } \\
\text { Schiller- } \\
\text { Universität }\end{array}$ & Jena & 17.07.14 & http://www.histinst.uni-jena.de/ \\
\hline Universität & Kassel & 17.07.14 & $\begin{array}{l}\text { http://www.uni- } \\
\text { kassel.de/fb05/fachgruppen/geschichte/mittelalter/umweltgeschichte.html }\end{array}$ \\
\hline $\begin{array}{l}\text { Christian- } \\
\text { Albrechts- } \\
\text { Universität }\end{array}$ & Kiel & 17.07 .14 & http://www.ecosystems.uni-kiel.de/abt umwelt.shtml \\
\hline Universität & $\begin{array}{l}\text { Koblenz- } \\
\text { Landau }\end{array}$ & 18.07.14 & http://www.uni-koblenz-landau.de/koblenz/fb2/geschi \\
\hline Universität & Köln & 18.07.14 & http://histinst.phil-fak.uni-koeln.de/ \\
\hline Universität & Konstanz & 18.07.14 & http://www.geschichte.uni-konstanz.de/willkommen/ \\
\hline Universität & Leipzig & 19.07.14 & http://www.gko.uni-leipzig.de/historisches-seminar/startseite.html \\
\hline $\begin{array}{l}\text { Johannes } \\
\text { Gutenberg- } \\
\text { Universität }\end{array}$ & Mainz & 19.07.14 & http://www.geschichte.uni-mainz.de/NeuereGeschichte/1103.php \\
\hline Universität & Mannheim & 19.07.14 & http://www.geschichte.uni-mannheim.de/startseite/ \\
\hline $\begin{array}{l}\text { Philipps- } \\
\text { Universität }\end{array}$ & Marburg & 19.07.14 & $\begin{array}{l}\text { http://www.uni- } \\
\text { marburg.de/fb06/ng/mitarbeiter/eckart conze/mitarbeiter/grabbe/index.html?searchter } \\
\text { m=umweltgeschichte }\end{array}$ \\
\hline $\begin{array}{l}\text { Ludwig- } \\
\text { Maximilians- } \\
\text { Universität }\end{array}$ & München & 19.07.14 & $\begin{array}{l}\text { http://www.geschichte.uni- } \\
\text { muenchen.de/personen/geschaeftsstelle/freytag/publ_freytag.html } \\
\text { http://www.gose.geschichte.uni- } \\
\text { muenchen.de/personen/professoren/hausmann_guido/index.html }\end{array}$ \\
\hline $\begin{array}{l}\text { Westfälische } \\
\text { Wilhelms- } \\
\text { Universität }\end{array}$ & Münster & 01.08.14 & http://www.uni-muenster.de/GeschichtePhilosophie/Institute/index.html \\
\hline $\begin{array}{l}\text { Carl von } \\
\text { Ossietzky- } \\
\text { Universität }\end{array}$ & Oldenburg & 01.08 .14 & http://www.uni-oldenburg.de/geschichte/das-institut/\# \\
\hline Universität & Osnabrück & 01.08 .14 & http://www.geschichte.uni-osnabrueck.de/historisches seminar/aktuelles.html \\
\hline Universität & Paderborn & 01.08 .14 & $\begin{array}{l}\text { http://kw.uni-paderborn.de/institute-einrichtungen/historisches- } \\
\text { institut/personal/faessler/ }\end{array}$ \\
\hline Universität & Passau & 01.08 .14 & $\begin{array}{l}\text { http://www.phil.uni-passau.de/fakultaet/departments/governance-und-historische- } \\
\text { wissenschaft.html }\end{array}$ \\
\hline Universität & Potsdam & 01.08 .14 & http://www.uni-potsdam.de/db/geschichte/ \\
\hline Universität & Regensburg & 01.08 .14 & $\begin{array}{l}\text { http://www.uni-regensburg.de/philosophie-kunst-geschichte- } \\
\text { gesellschaft/geschichte/fächer-und-teilfächer/index.html }\end{array}$ \\
\hline Universität & Rostock & 01.08 .14 & http://www.geschichte.uni-rostock.de/en/lehrende/apl-professoren-pds/borowy/ \\
\hline Universität & $\begin{array}{l}\text { des } \\
\text { Saarlandes }\end{array}$ & 01.08 .14 & http://www.uni-saarland.de/ m.grabas/ \\
\hline Universität & Siegen & 01.08 .14 & http://www.uni-siegen.de/phil/geschichte/ \\
\hline Universität & Trier & 04.08 .14 & https://www.uni-trier.de/index.php?id=36604\#c87060 \\
\hline Universität & Vechta & 04.08 .14 & $\begin{array}{l}\text { http://www.uni-vechta.de/einrichtungen/wissenschaftliche-einrichtungen/institute- } \\
\text { faecher/igk/geschichte/?L=title\%25 }\end{array}$ \\
\hline $\begin{array}{l}\text { Julius- } \\
\text { Maximilians- } \\
\text { Universität }\end{array}$ & Würzburg & 04.08 .14 & http://www.sinologie.uni-wuerzburg.de/struktur/personal/prof dr raimund th kolb/ \\
\hline $\begin{array}{l}\text { Bergische } \\
\text { Universität }\end{array}$ & Wuppertal & 04.08 .14 & http://www.geschichte.uni-wuppertal.de/ \\
\hline
\end{tabular}




\section{B Analysierte Texte}

Aguirre, Benigno E., Better Disaster Statistics: The Lisbon Earthquake, in: Journal of Interdisciplinary History (2012), S. 27-42.

Amate, Juan Infante et al, Erosion in the Mediterranean: The Case of Olive Groves in the South of Spain (1752-2000), in: Environmental History (2013), S. 360-382.

Bankoff, Greg, Bodies on the Beach: Domesticates and Disasters in the Spanish Philippines 1750-1898, in: Environment and History (2007), S. 285-306.

Bayerl, Günter, Das Umweltproblem und seine Wahrnehmung in der Geschichte, in: Jörg Calließ/Jörn Rüsen/Manfred Striegnitz (Hrsg.), Mensch und Umwelt in der Geschichte, Pfaffenweiler 1989, S. 47-96.

Bayerl, Günter, Umweltsanierung auf dem Gebiet der ehemaligen DDR: Ein Weg in die ökoindustrielle Gesellschaft?, in: Studienarchiv Umweltgeschichte (2009), S. 3-14.

Beattie, James, Environmental Anxiety in New Zealand, 1840-1941: Climate Change, Soil Erosion, Sand Drift, Flooding and Forest Conservation, in: Environment and History (2003), S. 379-392.

Bodner, Reinhard, Ein "Zusammenhang von oben nach unten?" Zur Kulturanalyse eines Felssturzereignisses und der Frage nach seinen Schuldigen, in: Patrick Masius/Jana Sprenger/Eva Mackowiak (Hrsg.), Katastrophen machen Geschichte. Umweltgeschichtliche Prozesse im Spannungsfeld von Ressourcennutzung und Extremereignis, Göttingen, Göttingen 2010, S. 173-196.

Bowlus, Charles R., Die Umweltkrise im Europa des 14. Jahrhunderts, in: Rolf Peter Sieferle (Hrsg.), Fortschritte der Naturzerstörung, Frankfurt am Main 1988, S. 13-30.

Brantz, Dorothee, 'Risky Business': Disease, Disaster and the Unintended Consequences of Epizootics in Eighteenth- and Nineteenth-Century France and Germany, in: Environment and History (2011), S. 35-51.

Bruno, Andy, Tumbling Snow: Vulnerability to Avalanches in the Soviet North, in: Environmental History (2013), S. 683-709.

Buchholz, Dietmar, Landnutzungsprobleme im „Großen Bruch“ zwischen Oschersleben und Hornburg - ein historischer Exkurs, in: Studienarchiv Umweltgeschichte (2012), S. 1325.

Buhs, Joshua Blu, Dead Cows on a Georgia Field: Mapping the Cultural Landscape of the Post-World War II American Pesticide Controversies, in: Environmental History (2007), S. 99-121.

Cao, Shuji/Li, Yushang/Yang, Bin, Mt. Tambora, Climatic Changes, and China's Decline in the Nineteenth Century, in: Journal of World History (2012), S. 587-607.

Carey, Mark, The history of ice: how glaciers became an endangered species, in: Environmental History (2007), S. 497-527.

Castonguay, Stéphane, The Production of Flood as Natural Catastrophe: Extreme Events and the Construction of Vulnerability in the Drainage Basin of the St. Francis River (Quebec), Mid-Nineteenth to Mid-Twentieth Century, in: Environmental History (2007), S. 820-844.

Crook, D. S./Siddle, D. J./Jones, R. T./Dearing, J. A./Foster, G. C./Thompson, R., Forestry and Flooding in the Annecy Petit Lac Catchment, Haute-Savoie 1730-2000, in: Environment and History (2002), S. 403-428.

Cyffka, Bernd, Wandel einer Naturlandschaft zu einer Industrielandschaft. Bergbau, Siedlungsentwicklung und Verhüttung auf der Halbinsel Kola, in: Bernd Herrmann 
(Hrsg.), Beiträge zum Göttinger Umwelthistorischen Kolloquium 2004 - 2006, Göttingen 2007, S. 59-78.

Daley, Ben/Griggs, Peter, 'Loved to Death': Coral Collecting in the Great Barrier Reef, Australia, 1770-1970, in: Environment and History (2008), S. 89-119.

Denning, Andrew, From Sublime Landscapes to "White Gold": How Skiing Transformed the Alps after 1930, in: Environmental History (2014), S. 78-108.

Engels, Jens Ivo, Gefährlicher Wasserstand im "Wirtschaftswunderland": Die Hamburger Sturmflut vom Februar 1962, in: Gerrit Jasper Schenk (Hrsg.), Katastrophen. Vom Untergang Pompejis bis zum Klimawandel, Ostfildern 2009, S. 171-181.

García Latorre, Juan/Sánchez Picón, Andrés/García Latorre, Jesús, The Man-Made Desert: Effects of Economic and Demographic Growth on the Ecosystems of Arid Southeastern Spain, in: Environmental History (2001), S. 75-94.

Griggs, Peter, Deforestation and Sugar Cane Growing in Eastern Australia, 1860-1995, in: Environment and History (2007), S. 255-283.

Huff, Tobias, „Jahr ohne Sommer" - Die Klimaanomalie von 1816 und ihre Folgen für Rheinhessen, in: Zeitschrift für Agrargeschichte und Agrarsoziologie (2010), S. 51-68.

Josephson, Paul, Umweltschäden des Kalten Krieges in der UdSSR, in: Bernd Greiner/Christian Th Müller/Claudia Weber (Hrsg.), Ökonomie im Kalten Krieg 2010, S. 326-347.

Knopf, Thomas, 'Schleichende Katastrophen' - Bodenübernutzung in vorindustriellen Gesellschaften, in: Patrick Masius/Jana Sprenger/Eva Mackowiak (Hrsg.), Katastrophen machen Geschichte. Umweltgeschichtliche Prozesse im Spannungsfeld von Ressourcennutzung und Extremereignis, Göttingen, Göttingen 2010, S. 31-46.

Kraft, Claudia, Zwischen Machbarkeitsphantasien und Apokalypse: Umweltgeschichte der Sowjetunion - Der Reaktorunfall in Tschernobyl im Jahr 1986, in: Bärbel Kuhn/Astrid Windus (Hrsg.), Umwelt und Klima im Geschichtsunterricht, St. Ingbert 2013, S. 77-91.

Kraker, Adriaan de, Storminess in the Low Countries, 1390-1725, in: Environment and History (2013), S. 149-171.

Mann, Michael, Ecological Change in North India: Deforestation and Agrarian Distress in the Ganga-Jamna Doab 1800-1850, in: Environment and History (1995), S. 201-220.

Mathews, Andrew Salvador, Suppressing Fire and Memory: Environmental Degradation and Political Restoration in the Sierra Juárez of Oaxaca, 1887-2001, in: Environmental History (2003), S. 77-108.

McClenachan, Loren, Recreation and the "Right to Fish" Movement: Anglers and Ecological Degradation in the Florida Keys, in: Environmental History (2013), S. 76-87.

McFarlane, Wallace Scot, Defining a Nuisance: Pollution, Science, and Environmental Politics on Maine's Androscoggin River, in: Environmental History (2012), S. 307-335.

Muscolino, Micah S., Free Content Violence Against People and the Land: The Environment and Refugee Migration from China's Henan Province, 1938-1945, in: Environment and History (2011), S. 291-311.

Obertreis, Julia, Eine "ökologische" Katastrophe in historischer Perspektive: Die Verlandung des Aralsees und die russländische und sowjetische Bewässerungs- und Baumwollanbaupolitik in Usbekistan und Turkmenistan seit der 2. Hälfte des 19. Jahrhunderts, in: Thoralf Klein/Reiner Prass/Susanne Rau/Lars Schladitz (Hrsg.), Umweltgeschichte in globaler Perspektive. Vortragsreihe des Historischen Seminars der Universität Erfurt im Sommersemester 2010, Erfurt 2013, S. 1-26.

Perri, Michael, 'Ruined and Lost': Spanish Destruction of the Pearl Coast in the Early Sixteenth Century, in: Environment and History (2009), S. 129-161. 
Pritchard, Sara B., An Envirotechnical Disaster: Nature, Technology, and Politics at Fukushima, in: Environmental History (2012), S. 219-243.

Rohr, Christian, Man and Natural Disaster in the Late Middle Ages: The Earthquake in Carinthia and Northern Italy on 25 January 1348 and its Perception, in: Environment and History (2003), S. 127-149.

Rohr, Christian, Zum Umgang mit Tierplagen im Alpenraum in der Frühen Neuzeit, in: Katharina Engelken/Dominik Hünniger/Steffi Windelen (Hrsg.), Beten, Impfen, Sammeln. Zur Viehseuchen- und Schädlingsbekämpfung in der Frühen Neuzeit ; [Ergebnis eines Workshops, den die HerausgeberInnen am 21. und 22. Juni 2006 in Göttingen unter dem Titel "Herausforderung Ressourcensicherung - Zur Viehseuchenund Schädlingsbekämpfung im 18. und 19. Jahrhundert" veranstalteten], Göttingen 2007, S. 99-134.

Romero, A./Baker, R./Cresswell, J. E./Singh, A./McKie, A./Manna, M., Environmental History of Marine Mammal Exploitation in Trinidad and Tobago, W.I., and its Ecological Impact, in: Environment and History (2002), S. 255-274.

Ross, Corey, The Tin Frontier: Mining, Empire, and Environment in Southeast Asia, 1870s1930s, in: Environmental History (2014), S. 454-479.

Schenk, Gerrit Jasper, Lektüren im Buch der Natur. Wahrnehmung, Beschreibung und Deutung von Naturkatastrophen, in: Susanne Rau/Birgit Studt/Stefan Benz (Hrsg.), Geschichte schreiben. Ein Quellen- und Studienhandbuch zur Historiografie (ca. 1350 1750), Berlin 2010, S. 507-521.

Scheringer, Martin, Umweltchemikalien 50 Jahre nach Silent Spring: ein ungelöstes Problem, in: GAIA (2012), S. 210-216.

Schott, Dieter, Energie und Stadt in Europa. Von der vorindustriellen Holznot bis zur Ölkrise der 1970er Jahre. Einführung, in: Dieter Schott (Hrsg.), Energie und Stadt in Europa. Von der vorindustriellen "Holznot" bis zur Ölkrise der 1970er Jahre ; Beiträge auf der 3. Internationalen Stadtgeschichts-Konferenz in Budapest $1996=$ Energy and the city in Europe, Stuttgart 1997, S. 7-42.

Schott, Dieter, One City - Three Catastrophies: Hamburg from the great fire 1842 to the Great Flood 1962, in: Geneviève Massard-Guilbaud (Hrsg.), Cities and catastrophes. Coping with emergency in European history = Villes et catastrophes, Frankfurt am Main 2002, S. 185-204.

Schulze, Bernd/Grossmann, Günter, Probleme des Umweltschutzes in Erfurt im 19. Jahrhundert, in: Studienarchiv Umweltgeschichte (2013), S. 3-11.

Sheail, John, Pesticides and the British Environment: An Agricultural Perspective, in: Environment and History (2013), S. 87-108.

Shulman, Seth, Ökologische Schäden des Kalten Krieges in den USA, in: Bernd Greiner/Christian Th Müller/Claudia Weber (Hrsg.), Ökonomie im Kalten Krieg 2010, S. 242-259.

Singh, M., Basutoland: A Historical Journey into the Environment, in: Environment and History (2000), S. 31-70.

Slavin, Philip, Warfare and Ecological Destruction in Early Fourteenth-Century British Isles, in: Environmental History (2014), S. 528-550.

Smith, S. D., Storm Hazard and Slavery: The Impact of the 1831 Great Caribbean Hurricane on St Vincent, in: Environment and History (2012), S. 97-123.

Studnicki-Gizbert, Daviken/Schecter, David, The Environmental Dynamics of a Colonial FuelRush: Silver Mining and Deforestation in New Spain, 1522 to 1810, in: Environmental History (2010), S. 94-119. 
Swanson, Drew A., Endangered Species and Threatened Landscapes in Appalachia: Managing the Wild and the Human in the American Mountain South, in: Environment and History (2012), S. 35-60.

Tully, John, A Victorian Ecological Disaster: Imperialism, the Telegraph, and Gutta-Percha, in: Journal of World History (2009), S. 559-579.

Völkel, Franziska, "Aes rarum" - Zur Bedeutung des Silberbergbaus in der Oberharzer Stadt Sankt Andreasberg, in: Manfred Jakubowski-Tiessen/Patrick Masius/Jana Sprenger (Hrsg.), Schauplätze der Umweltgeschichte in Niedersachsen ; Werkstattbericht, Göttingen, Göttingen 2014, S. 103-118.

Worster, Donald, "Dust Bowl". Dürre und Winderosion in amerikanischen Südwesten, in: Rolf Peter Sieferle (Hrsg.), Fortschritte der Naturzerstörung, Frankfurt am Main 1988, S. 118-157. 


\section{Ranglisten der Werte}

\begin{tabular}{|l|c|c|c|c|c|c|c|c|}
\hline \multirow{2}{*}{ Nach Dokumenten } & \multicolumn{3}{|c|}{ Rang } & \multicolumn{3}{|c|}{ Anteil Summe Dokumente $\%$} \\
\cline { 2 - 9 } & Gesamt & D & USA & UK & Gesamt & D & USA & UK \\
\hline ANT/ÖK - Ökonomie & 1 & 4 & 1 & 1 & $69 \%$ & $50 \%$ & $79 \%$ & $100 \%$ \\
\hline ANT/GE/KR - Krankheit, Verletzungen & 2 & 1 & 2 & 2 & $54 \%$ & $65 \%$ & $50 \%$ & $75 \%$ \\
\hline ANT/ZH - Zuhause, Eigentum & 3 & 3 & 5 & 5 & $48 \%$ & $55 \%$ & $36 \%$ & $50 \%$ \\
\hline ANT/LG/NA/BO - Boden, Felder & 4 & 2 & 15 & 4 & $46 \%$ & $55 \%$ & $14 \%$ & $63 \%$ \\
\hline ANT/GE/TO - Tod & 5 & 5 & 3 & 8 & $44 \%$ & $50 \%$ & $50 \%$ & $38 \%$ \\
\hline ANT/LG/NA/NM - Nahrungsmittel & 6 & 7 & 11 & 3 & $37 \%$ & $45 \%$ & $21 \%$ & $63 \%$ \\
\hline ANT/EQ - Einkommensquelle & 7 & 8 & 4 & 11 & $35 \%$ & $40 \%$ & $43 \%$ & $25 \%$ \\
\hline ANT/LG/WA - Wasser & 8 & 6 & 10 & 19 & $31 \%$ & $50 \%$ & $21 \%$ & $13 \%$ \\
\hline AB - Artenbestände & 9 & 12 & 7 & 9 & $30 \%$ & $20 \%$ & $29 \%$ & $38 \%$ \\
\hline ANT/LG/NA/ER - Ernte, Saatgut & 10 & 11 & 6 & 6 & $26 \%$ & $20 \%$ & $29 \%$ & $50 \%$ \\
\hline ANT/SCÖ - Schönheit der Natur & 11 & 9 & 16 & 15 & $24 \%$ & $25 \%$ & $14 \%$ & $25 \%$ \\
\hline ÖS - Ökosystem & 12 & 15 & 12 & 13 & $22 \%$ & $15 \%$ & $21 \%$ & $25 \%$ \\
\hline ANT/IS - Infrastruktur & 13 & 17 & 9 & 12 & $22 \%$ & $15 \%$ & $29 \%$ & $25 \%$ \\
\hline ANT/RL - Rohstofflieferant & 14 & 14 & 21 & 10 & $22 \%$ & $15 \%$ & $7 \%$ & $38 \%$ \\
\hline TI - Tiere & 15 & 18 & 13 & 7 & $19 \%$ & $10 \%$ & $21 \%$ & $50 \%$ \\
\hline AV - Artenvielfalt & 16 & 13 & 20 & 22 & $17 \%$ & $20 \%$ & $7 \%$ & $13 \%$ \\
\hline ANT/EF - Erholung, Freizeit, Komfort & 17 & 16 & 8 & 18 & $17 \%$ & $15 \%$ & $29 \%$ & $13 \%$ \\
\hline ANT/GE/HU - Hunger & 18 & 22 & 14 & 14 & $13 \%$ & $5 \%$ & $21 \%$ & $25 \%$ \\
\hline ANT/ID - Identität & 19 & 20 & 22 & 23 & $11 \%$ & $10 \%$ & $7 \%$ & $13 \%$ \\
\hline NA - Nachhaltigkeit & 20 & 26 & 24 & 16 & $9 \%$ & $0 \%$ & $7 \%$ & $25 \%$ \\
\hline UN - Unberührte Natur & 21 & 23 & 17 & 20 & $9 \%$ & $5 \%$ & $14 \%$ & $13 \%$ \\
\hline ANT/LG/LU - Luft & 22 & 10 & 25 & 27 & $9 \%$ & $25 \%$ & $0 \%$ & $0 \%$ \\
\hline ANT/MO - Moralische Verantwortung, Pflicht & 23 & 25 & 18 & 25 & $7 \%$ & $5 \%$ & $14 \%$ & $0 \%$ \\
\hline ANT/IM - Imageschaden & 24 & 19 & 26 & 17 & $7 \%$ & $10 \%$ & $0 \%$ & $25 \%$ \\
\hline ANT/WI - Wissen, Erkenntnis & 25 & 27 & 19 & 21 & $6 \%$ & $0 \%$ & $14 \%$ & $13 \%$ \\
\hline ANT/RE - Religion, Glaube & 26 & 24 & 27 & 24 & $6 \%$ & $5 \%$ & $0 \%$ & $13 \%$ \\
\hline ANT/TR - Tradition & 27 & 21 & 23 & 26 & $6 \%$ & $10 \%$ & $7 \%$ & $0 \%$ \\
\hline ANT/ST - Stabilität (polit.) & 28 & 28 & 28 & 28 & $2 \%$ & $0 \%$ & $0 \%$ & $0 \%$ \\
\hline
\end{tabular}

Rangliste der Werte nach Anzahl der Dokumente 


\begin{tabular}{|l|c|c|c|c|c|c|c|c|}
\hline \multirow{2}{*}{ Nach Codierungen } & \multicolumn{4}{|l|}{ Rang } & \multicolumn{3}{|c|}{ Anteil Summe Codierungen $\%$} \\
\cline { 2 - 9 } & Gesamt & D & USA & UK & Gesamt & D & USA & UK \\
\hline ANT/ÖK - Ökonomie & 1 & 4 & 2 & 1 & $14 \%$ & $9 \%$ & $13 \%$ & $18 \%$ \\
\hline ANT/GE/KR - Krankheit, Verletzungen & 2 & 1 & 1 & 6 & $11 \%$ & $13 \%$ & $15 \%$ & $7 \%$ \\
\hline ANT/ZH - Zuhause, Eigentum & 3 & 2 & 6 & 5 & $8 \%$ & $11 \%$ & $6 \%$ & $7 \%$ \\
\hline ANT/LG/NA/BO - Boden, Felder & 4 & 5 & 8 & 3 & $8 \%$ & $8 \%$ & $5 \%$ & $10 \%$ \\
\hline ANT/GE/TO - Tod & 5 & 7 & 3 & 8 & $7 \%$ & $5 \%$ & $12 \%$ & $3 \%$ \\
\hline TI - Tiere & 6 & 15 & 5 & 2 & $5 \%$ & $2 \%$ & $6 \%$ & $14 \%$ \\
\hline ANT/SCÖ - Schönheit der Natur & 7 & 12 & 13 & 4 & $5 \%$ & $3 \%$ & $3 \%$ & $8 \%$ \\
\hline ANT/LG/WA - Wasser & 8 & 3 & 11 & 20 & $4 \%$ & $10 \%$ & $3 \%$ & $1 \%$ \\
\hline ANT/LG/NA/NM - Nahrungsmittel & 9 & 6 & 12 & 7 & $4 \%$ & $6 \%$ & $3 \%$ & $5 \%$ \\
\hline AB - Artenbestände & 10 & 9 & 7 & 15 & $4 \%$ & $4 \%$ & $6 \%$ & $2 \%$ \\
\hline ANT/EQ - Einkommensquelle & 11 & 8 & 9 & 9 & $4 \%$ & $5 \%$ & $4 \%$ & $3 \%$ \\
\hline ANT/EF - Erholung, Freizeit, Komfort & 12 & 13 & 4 & 14 & $3 \%$ & $3 \%$ & $6 \%$ & $2 \%$ \\
\hline ANT/IS - Infrastruktur & 13 & 16 & 10 & 16 & $3 \%$ & $2 \%$ & $4 \%$ & $2 \%$ \\
\hline ÖS - Ökosystem & 14 & 14 & 14 & 12 & $3 \%$ & $3 \%$ & $3 \%$ & $3 \%$ \\
\hline AV - Artenvielfalt & 15 & 10 & 15 & 21 & $3 \%$ & $4 \%$ & $2 \%$ & $1 \%$ \\
\hline ANT/LG/NA/ER - Ernte, Saatgut & 16 & 17 & 16 & 10 & $2 \%$ & $2 \%$ & $2 \%$ & $3 \%$ \\
\hline ANT/RL - Rohstofflieferant & 17 & 18 & 23 & 11 & $2 \%$ & $2 \%$ & $0 \%$ & $3 \%$ \\
\hline UN - Unberührte Natur & 18 & 22 & 17 & 19 & $2 \%$ & $0 \%$ & $2 \%$ & $1 \%$ \\
\hline ANT/GE/HU - Hunger & 19 & 23 & 18 & 13 & $1 \%$ & $0 \%$ & $1 \%$ & $3 \%$ \\
\hline ANT/LG/LU - Luft & 20 & 11 & 25 & 27 & $1 \%$ & $4 \%$ & $0 \%$ & $0 \%$ \\
\hline NA - Nachhaltigkeit & 21 & 26 & 21 & 17 & $1 \%$ & $0 \%$ & $1 \%$ & $2 \%$ \\
\hline ANT/IM - Imageschaden & 22 & 19 & 26 & 18 & $1 \%$ & $2 \%$ & $0 \%$ & $2 \%$ \\
\hline ANT/ID - Identität & 23 & 20 & 19 & 22 & $1 \%$ & $1 \%$ & $1 \%$ & $1 \%$ \\
\hline ANT/MO - Moralische Verantwortung, Pflicht & 24 & 24 & 20 & 25 & $1 \%$ & $0 \%$ & $1 \%$ & $0 \%$ \\
\hline ANT/WI - Wissen, Erkenntnis & 25 & 27 & 22 & 23 & $0 \%$ & $0 \%$ & $1 \%$ & $1 \%$ \\
\hline ANT/RE - Religion, Glaube & 26 & 25 & 27 & 24 & $0 \%$ & $0 \%$ & $0 \%$ & $1 \%$ \\
\hline ANT/TR - Tradition & 27 & 21 & 24 & 26 & $0 \%$ & $1 \%$ & $0 \%$ & $0 \%$ \\
\hline ANT/ST - Stabilität (polit.) & 28 & 28 & 28 & 28 & $0 \%$ & $0 \%$ & $0 \%$ & $0 \%$ \\
\hline
\end{tabular}

Rangliste der Werte nach Anzahl der Codierungen 


\begin{tabular}{|l|c|c|c|c|c|c|c|c|}
\hline \multirow{2}{*}{$\begin{array}{l}\text { Gewichtet } \\
\text { (Dokumente 2/3, Codierungen 1/3) }\end{array}$} & \multicolumn{3}{|c|}{ Rang } & \multicolumn{3}{c|}{ Gewichteter $\%$-Wert } \\
\cline { 2 - 9 } & Gesamt & D & USA & UK & Gesamt & D & USA & UK \\
\hline ANT/ÖK - Ökonomie & 1 & 5 & 1 & 1 & $50 \%$ & $36 \%$ & $57 \%$ & $73 \%$ \\
\hline ANT/GE/KR - Krankheit, Verletzungen & 2 & 1 & 2 & 2 & $39 \%$ & $48 \%$ & $38 \%$ & $52 \%$ \\
\hline ANT/ZH - Zuhause, Eigentum & 3 & 2 & 5 & 6 & $35 \%$ & $40 \%$ & $26 \%$ & $36 \%$ \\
\hline ANT/LG/NA/BO - Boden, Felder & 4 & 3 & 15 & 3 & $34 \%$ & $39 \%$ & $11 \%$ & $45 \%$ \\
\hline ANT/GE/TO - Tod & 5 & 6 & 3 & 8 & $32 \%$ & $35 \%$ & $37 \%$ & $26 \%$ \\
\hline ANT/LG/NA/NM - Nahrungsmittel & 6 & 7 & 12 & 4 & $26 \%$ & $32 \%$ & $15 \%$ & $43 \%$ \\
\hline ANT/EQ - Einkommensquelle & 7 & 8 & 4 & 12 & $25 \%$ & $28 \%$ & $30 \%$ & $18 \%$ \\
\hline ANT/LG/WA - Wasser & 8 & 4 & 11 & 20 & $22 \%$ & $37 \%$ & $15 \%$ & $9 \%$ \\
\hline AB - Artenbestände & 9 & 11 & 7 & 10 & $21 \%$ & $15 \%$ & $21 \%$ & $26 \%$ \\
\hline ANT/LG/NA/ER - Ernte, Saatgut & 10 & 13 & 9 & 7 & $18 \%$ & $14 \%$ & $20 \%$ & $34 \%$ \\
\hline ANT/SCÖ - Schönheit der Natur & 11 & 10 & 16 & 11 & $18 \%$ & $18 \%$ & $10 \%$ & $19 \%$ \\
\hline ANT/IS - Infrastruktur & 12 & 16 & 8 & 15 & $16 \%$ & $11 \%$ & $20 \%$ & $17 \%$ \\
\hline ÖS - Ökosystem & 13 & 14 & 13 & 13 & $16 \%$ & $11 \%$ & $15 \%$ & $18 \%$ \\
\hline ANT/RL - Rohstofflieferant & 14 & 17 & 23 & 9 & $15 \%$ & $11 \%$ & $5 \%$ & $26 \%$ \\
\hline TI - Tiere & 15 & 18 & 10 & 5 & $14 \%$ & $7 \%$ & $16 \%$ & $38 \%$ \\
\hline ANT/EF - Erholung, Freizeit, Komfort & 16 & 15 & 6 & 18 & $12 \%$ & $11 \%$ & $21 \%$ & $9 \%$ \\
\hline AV - Artenvielfalt & 17 & 12 & 20 & 22 & $12 \%$ & $15 \%$ & $5 \%$ & $9 \%$ \\
\hline ANT/GE/HU - Hunger & 18 & 22 & 14 & 14 & $9 \%$ & $3 \%$ & $15 \%$ & $18 \%$ \\
\hline ANT/ID - Identität & 19 & 20 & 21 & 23 & $8 \%$ & $7 \%$ & $5 \%$ & $9 \%$ \\
\hline UN - Unberührte Natur & 20 & 23 & 17 & 19 & $7 \%$ & $3 \%$ & $10 \%$ & $9 \%$ \\
\hline ANT/LG/LU - Luft & 21 & 9 & 25 & 27 & $7 \%$ & $18 \%$ & $0 \%$ & $0 \%$ \\
\hline NA - Nachhaltigkeit & 22 & 26 & 22 & 16 & $7 \%$ & $0 \%$ & $5 \%$ & $17 \%$ \\
\hline ANT/IM - Imageschaden & 23 & 19 & 26 & 17 & $5 \%$ & $7 \%$ & $0 \%$ & $17 \%$ \\
\hline ANT/MO - Moralische Verantwortung, Pflicht & 24 & 24 & 18 & 25 & $5 \%$ & $3 \%$ & $10 \%$ & $0 \%$ \\
\hline ANT/MI - Wissen, Erkenntnis & 25 & 27 & 19 & 21 & $4 \%$ & $0 \%$ & $10 \%$ & $9 \%$ \\
\hline ANT/RE - Religion, Glaube & 26 & 25 & 27 & 24 & $4 \%$ & $3 \%$ & $0 \%$ & $9 \%$ \\
\hline ANT/TR - Tradition & 27 & 21 & 24 & 26 & $4 \%$ & $7 \%$ & $5 \%$ & $0 \%$ \\
\hline ANT/ST - Stabilität (polit.) & 28 & 28 & 28 & 28 & $1 \%$ & $0 \%$ & $0 \%$ & $0 \%$ \\
\hline
\end{tabular}

Rangliste der Werte gewichtet nach Anteil Dokumente und Anteil Codierungen 


\section{Wertekategoriegruppen - Zuordnung der Werte}

\begin{tabular}{|c|c|c|c|c|c|c|}
\hline Wertekategorie & $\begin{array}{c}\text { Use Values / } \\
\text { Non-use Values }\end{array}$ & $\begin{array}{l}\text { materiell/ } \\
\text { immateriell }\end{array}$ & $\begin{array}{l}\text { Existenzialw.l } \\
\text { Nicht-Existenz. }\end{array}$ & \multicolumn{3}{|c|}{$\begin{array}{l}\text { Summe } \\
\text { Codierungen }\end{array}$} \\
\hline $\mathrm{TI}$ - Tiere & Indirect Use & $\mathrm{i}$ & \multirow{6}{*}{$\begin{array}{l}\mathrm{n} \\
\mathrm{n} \\
\mathrm{n} \\
\mathrm{n} \\
\mathrm{n} \\
\mathrm{n}\end{array}$} & 48 & & \multirow{6}{*}{158} \\
\hline ÖS - Ökosystem & Indirect Use & $\mathrm{i}$ & & 26 & & \\
\hline$A B$ - Artenbestände & Indirect Use & $\mathrm{i}$ & & 37 & & \\
\hline AV - Artenvielfalt & Indirect Use & $\mathrm{i}$ & & 22 & & \\
\hline NA - Nachhaltigkeit & Indirect Use & $\mathrm{i}$ & & 9 & & \\
\hline UN - Unberührte Natur & Non-Use & $\mathrm{i}$ & & 16 & & \\
\hline ANT - SCÖ - Schönheit der Natur & Non-Use & $\mathrm{i}$ & \multirow{10}{*}{$\begin{array}{l}\mathrm{n} \\
\mathrm{n} \\
\mathrm{n} \\
\mathrm{n} \\
\mathrm{n} \\
\mathrm{n} \\
\mathrm{n} \\
\mathrm{n} \\
\mathrm{n} \\
\mathrm{n}\end{array}$} & 43 & & \multirow{22}{*}{716} \\
\hline ANT - IS - Infrastruktur & Direct Use & $\mathrm{m}$ & & 27 & & \\
\hline ANT - WI - Wissen, Erkenntnis & Indirect Use & $\mathrm{i}$ & & 3 & & \\
\hline ANT - MO - Moralische Verantwortung, Pflicht & Non-Use & $\mathrm{i}$ & & 5 & & \\
\hline ANT - RE - Religion, Glaube & Non-Use & $\mathrm{i}$ & & 3 & & \\
\hline ANT - ÖK - Ökonomie & Direct Use & $\mathrm{m}$ & & 118 & & \\
\hline ANT - IM - Imageschaden & Indirect Use & $\mathrm{i}$ & & 8 & & \\
\hline ANT - ST - Stabilität (polit.) & Indirect Use & $\mathrm{i}$ & & 1 & & \\
\hline ANT - TR - Tradition & Non-Use & $\mathrm{i}$ & & 3 & & \\
\hline ANT - ID - Identität & Non-Use & i & & 7 & & \\
\hline ANT - GE - GesundheitlTO - Tod & Direct Use & n.a. & \multirow{3}{*}{$\begin{array}{l}\mathrm{e} \\
\mathrm{e} \\
\mathrm{e}\end{array}$} & 59 & \multirow{3}{*}{165} & \\
\hline ANT - GE - GesundheitlKR - Krankheit, Verletzungen & Direct Use & n.a. & & 96 & & \\
\hline ANT - GE - GesundheitlHU - Hunger & Direct Use & n.a. & & 10 & & \\
\hline ANT - ZH - Zuhause, Eigentum & Direct Use & $\mathrm{m}$ & \multirow{4}{*}{$\begin{array}{l}\mathrm{n} \\
\mathrm{n} \\
\mathrm{n} \\
\mathrm{n}\end{array}$} & 74 & & \\
\hline ANT - EF - Erholung, Freizeit, Komfort & Direct Use & $\mathrm{i}$ & & 29 & & \\
\hline ANT - RL - Rohstofflieferant & Direct Use & $\mathrm{m}$ & & 17 & & \\
\hline ANT - EQ - Einkommensquelle & Direct Use & $\mathrm{m}$ & & 35 & & \\
\hline ANT - LG - LebensgrundlagelNA - NahrungINM - Nahrungsmittel & Direct Use & $\mathrm{m}$ & \multirow{3}{*}{$\begin{array}{l}\mathrm{e} \\
\mathrm{e} \\
\mathrm{e}\end{array}$} & 37 & \multirow{5}{*}{178} & \\
\hline ANT - LG - LebensgrundlagelNA - NahrunglER - Ernte, Saatgut & Direct Use & $\mathrm{m}$ & & 21 & & \\
\hline ANT - LG - LebensgrundlagelNA - Nahrung\BO - Boden, Felder & Direct Use & $\mathrm{m}$ & & 72 & & \\
\hline ANT - LG - LebensgrundlagelWA - Wasser & Direct Use & $\mathrm{m}$ & \multirow{2}{*}{$\begin{array}{l}\mathrm{e} \\
\mathrm{e}\end{array}$} & 38 & & \\
\hline ANT - LG - LebensgrundlagelLU - Luft & Direct Use & $\mathrm{m}$ & & 10 & & \\
\hline
\end{tabular}

\begin{tabular}{|c|c|c|c|c|}
\hline Wertekategoriegruppe & $\begin{array}{c}\text { Summe } \\
\text { Codierungen }\end{array}$ & Anteil (\%) & $\begin{array}{c}\text { Anzahl } \\
\text { Dokumente }\end{array}$ & Anteil (\%) \\
\hline Anthropozentrisch & 716 & $82 \%$ & 53 & $98 \%$ \\
\hline Nicht-anthropozentrisch & 158 & $18 \%$ & 32 & $59 \%$ \\
\hline Materiell & 449 & $51 \%$ & 51 & $94 \%$ \\
\hline Immateriell & 260 & $30 \%$ & 41 & $76 \%$ \\
\hline Gesundheit & 165 & $19 \%$ & 36 & $67 \%$ \\
\hline Existenzialwerte & 343 & $39 \%$ & 48 & $89 \%$ \\
\hline Nicht-Existenzialwerte & 531 & $61 \%$ & 54 & $100 \%$ \\
\hline Use Values & 797 & $91 \%$ & 54 & $100 \%$ \\
\hline Non-use Values & 77 & $9 \%$ & 21 & $39 \%$ \\
\hline Direct Use Values & 643 & $74 \%$ & 53 & $98 \%$ \\
\hline Indirect Use Values & 154 & $18 \%$ & 32 & $59 \%$ \\
\hline
\end{tabular}




\section{E Eidesstattliche Erklärung}

Hiermit erkläre ich an Eides statt, dass ich die eingereichte Dissertation (Werte in umweltgeschichtlichen Texten - Eine Analyse anhand des Begriffs Umweltschaden) selbständig und ohne unerlaubte Hilfe verfasst habe. Anderer als der von mir angegebenen Hilfsmittel und Schriften habe ich mich nicht bedient. Alle wörtlich oder sinngemäß den Schriften anderer Autorinnen oder Autoren entnommenen Stellen habe ich kenntlich gemacht. Die Abhandlung ist noch nicht veröffentlicht worden und noch nicht Gegenstand eines Promotionsverfahrens gewesen.

Göttingen, den 06.07.2017 